Supporting Information for

\title{
Validation of the CoGEF Method as a Predictive Tool for Polymer Mechanochemistry
}

\author{
Isabel M. Klein, ${ }^{\ddagger}$ Corey C. Husic, ${ }^{\ddagger}$ Dávid P. Kovács, ${ }^{\dagger}$ Nicolas J. Choquette, and Maxwell J. Robb* \\ Division of Chemistry and Chemical Engineering, California Institute of Technology, Pasadena, CA \\ 91125, United States
}

*These authors contributed equally. 'Present address: Cavendish Laboratory, University of Cambridge, Cambridge, United Kingdom.

*Email: mrobb@caltech.edu

\section{Table of Contents}

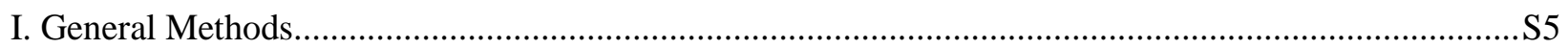

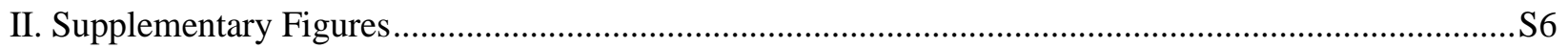

III. Summaries of Individual CoGEF Calculations .......................................................................

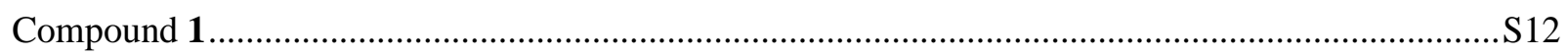

Compound 2 .

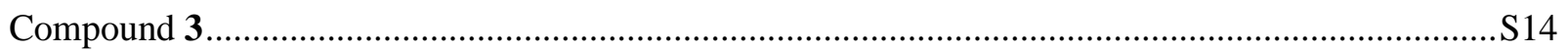

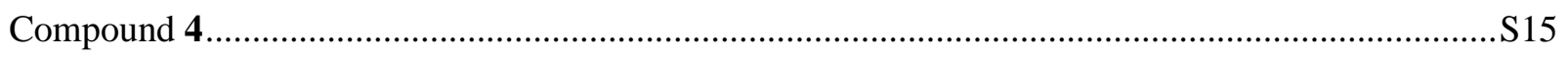

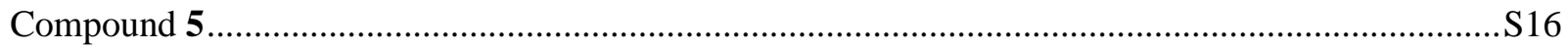

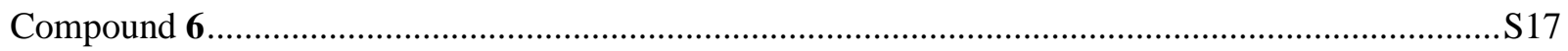

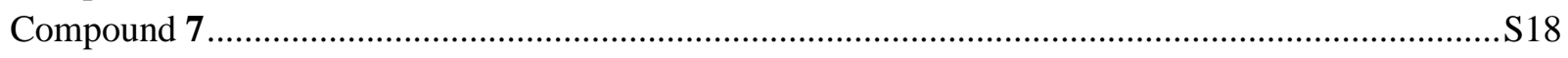

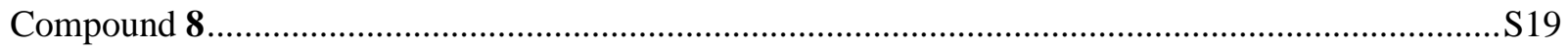

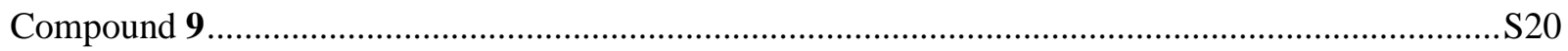

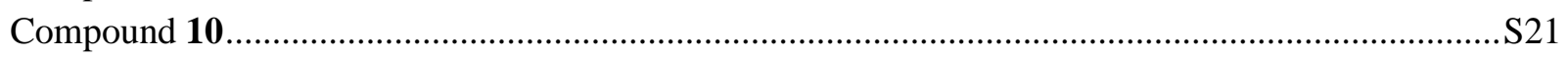

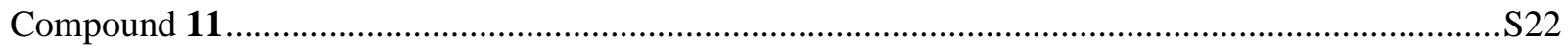

Compound 12

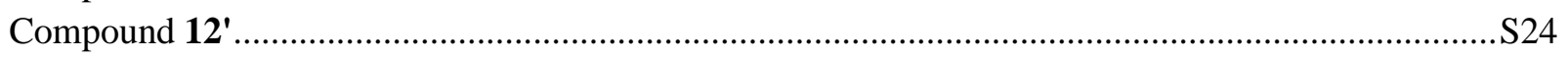

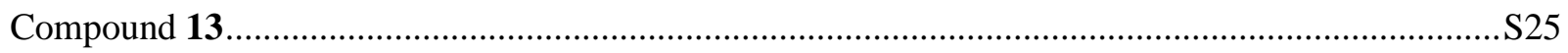

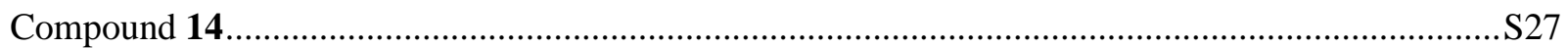

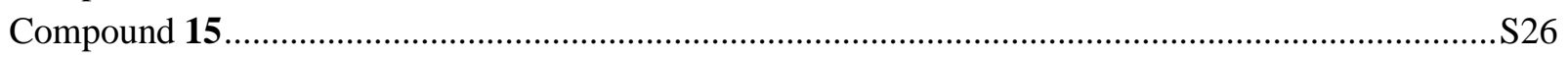

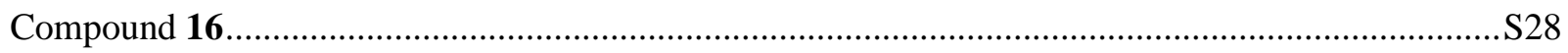

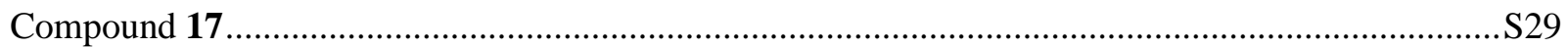

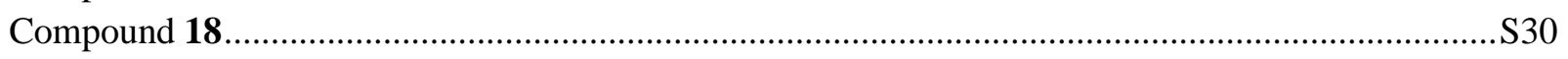




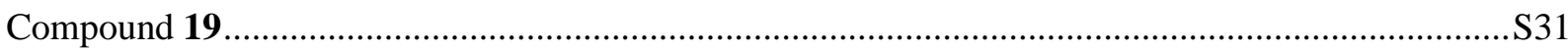

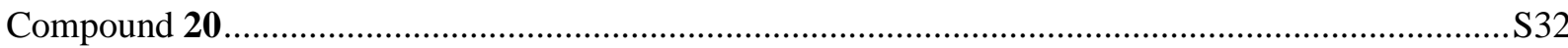

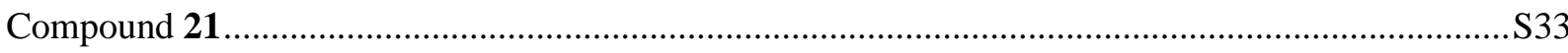

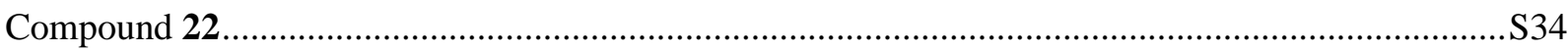

Compound 23

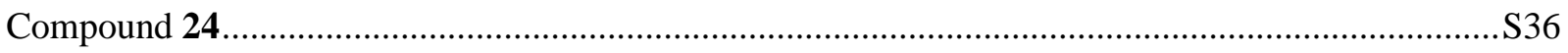

Compound 25

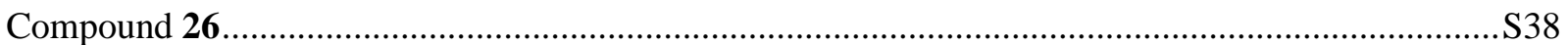

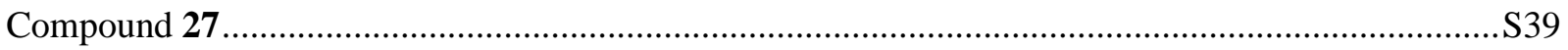

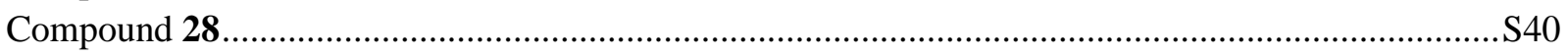

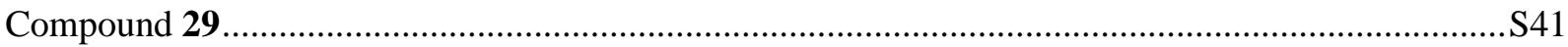

Compound 30

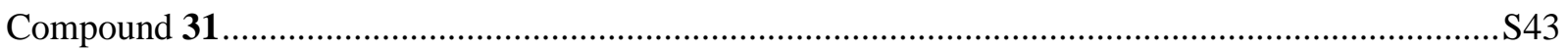

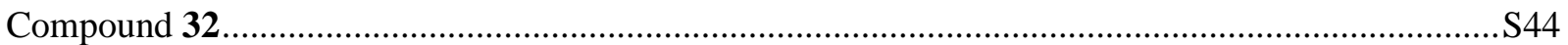

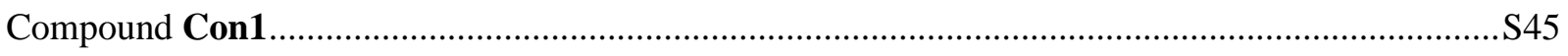

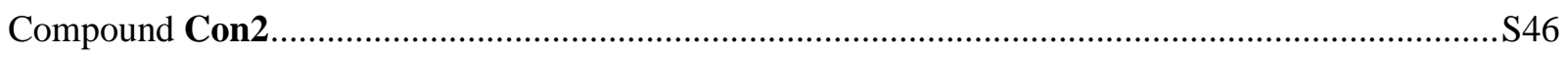

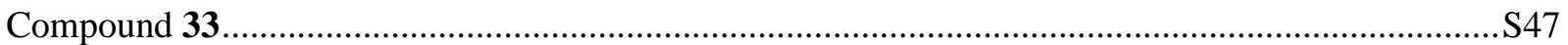

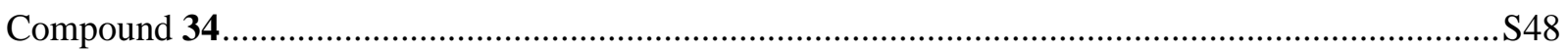

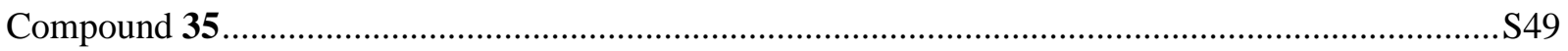

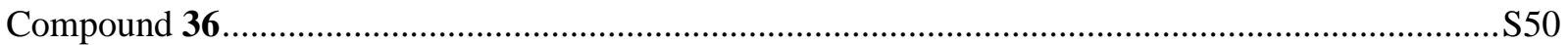

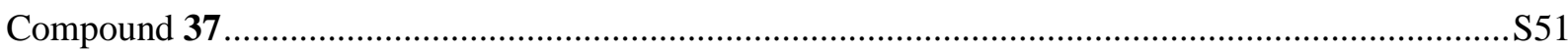

Compound 38

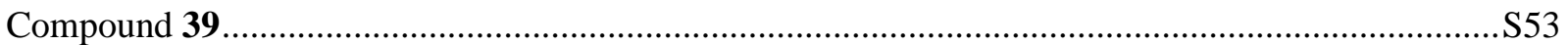

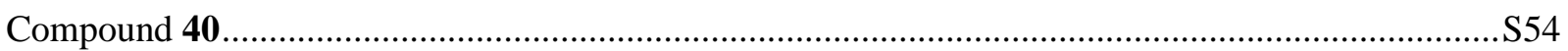

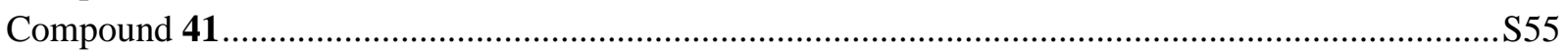

Compound 42

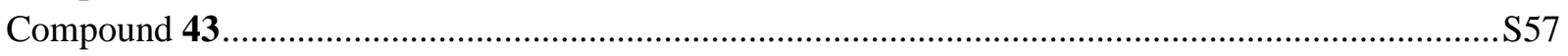

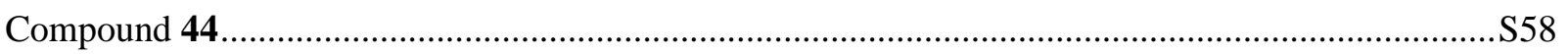

Compound 45

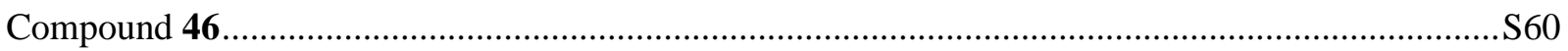

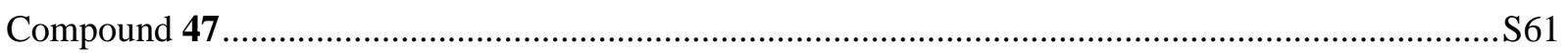

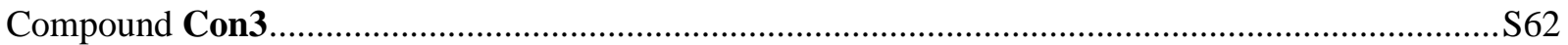

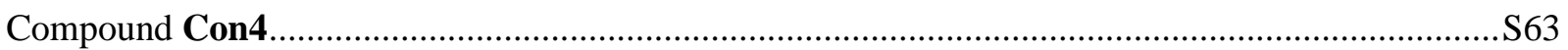

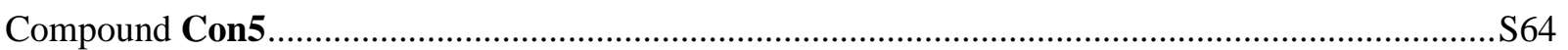

Compound 48

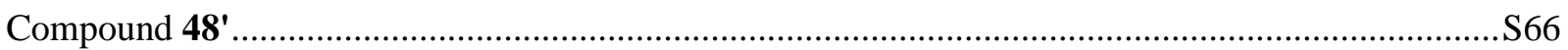

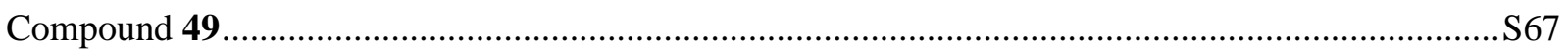

Compound 49'

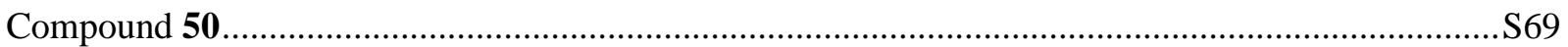

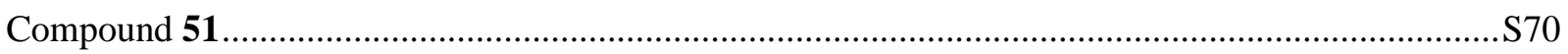

Compound 52

Compound 53

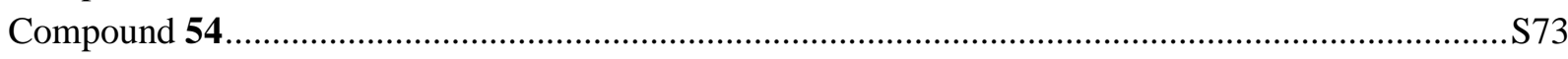

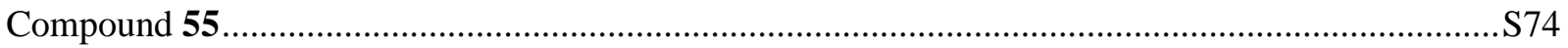


Compound 56

Compound 57

Compound 58

Compound 59

Compound 60 S79

Compound 61 $\mathrm{S} 80$

Compound 62 . S81

Compound 63 S82

Compound 64 $\mathrm{S} 83$

Compound 65. .S84

Compound 66 S85

Compound 67 S86

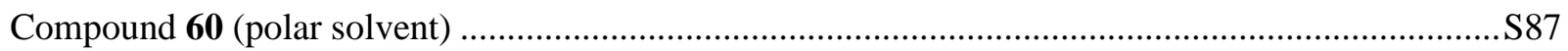

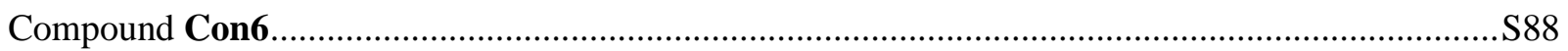

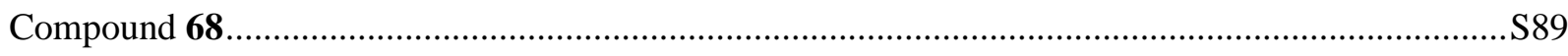

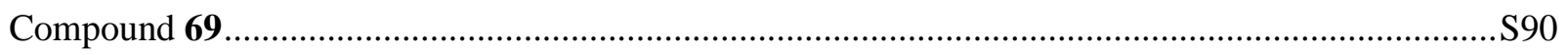

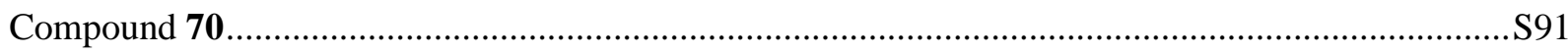

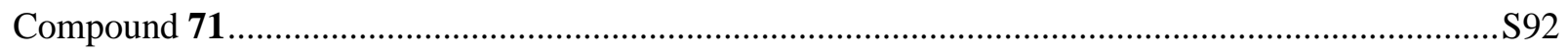

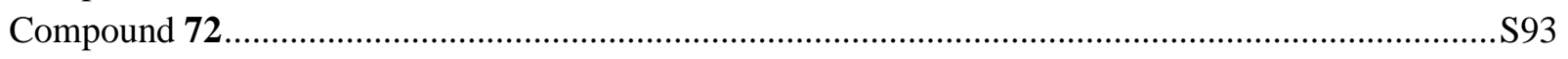

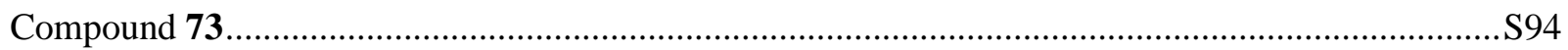

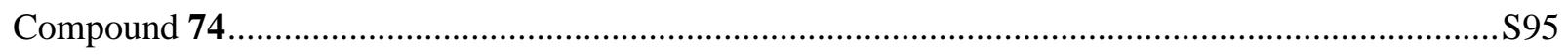

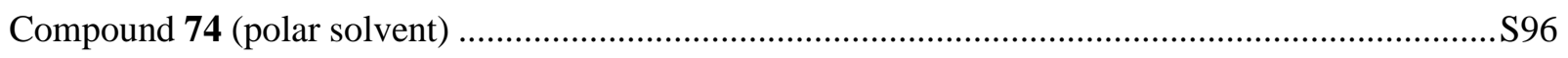

Compound 75

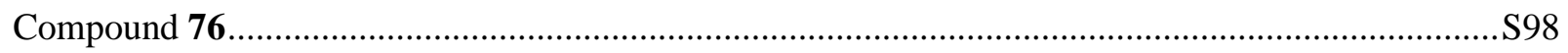

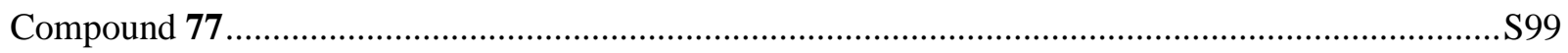

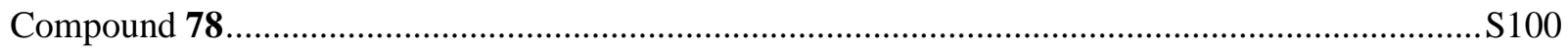

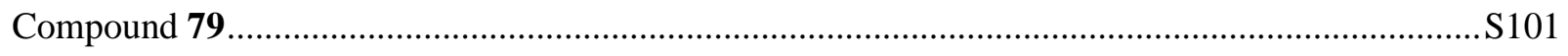

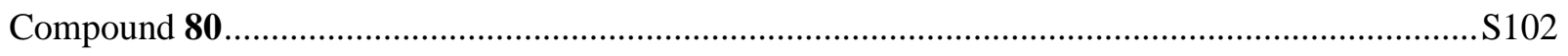

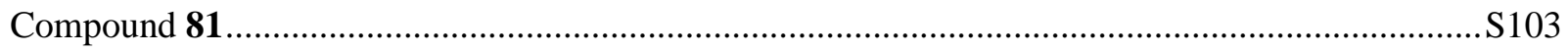

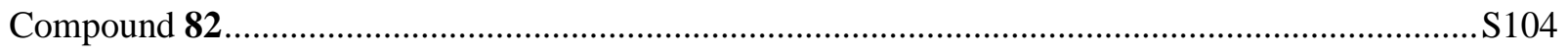

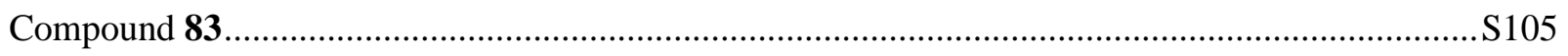

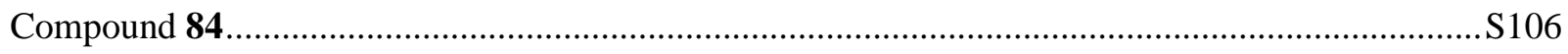

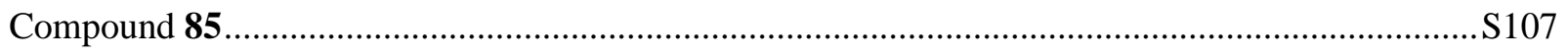

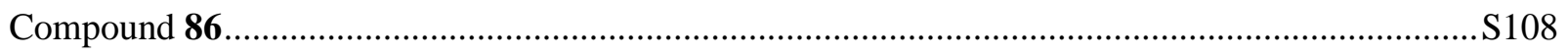

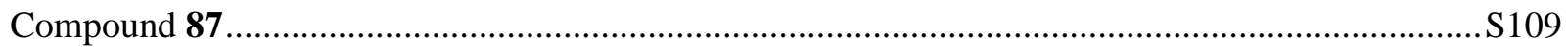

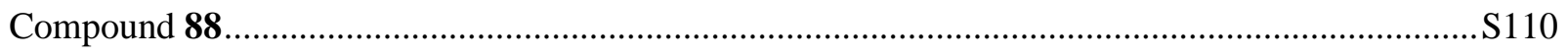

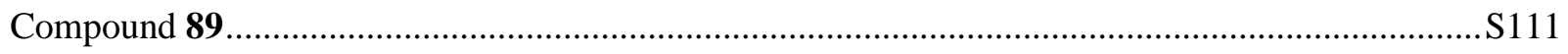

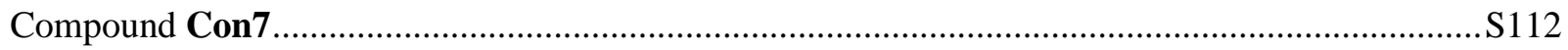

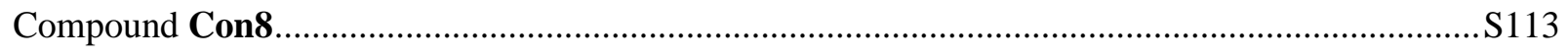

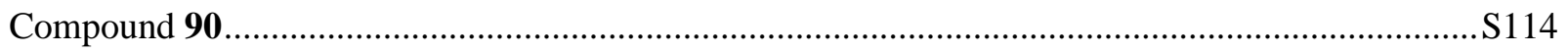

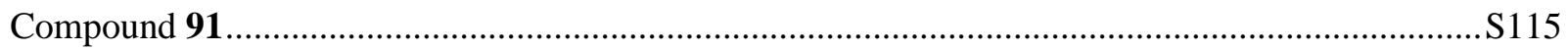

Compound 92

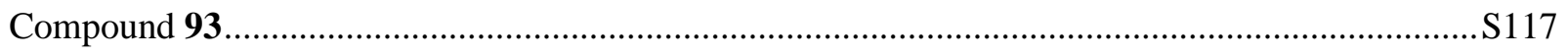

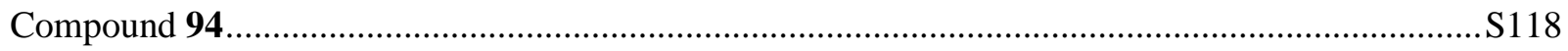




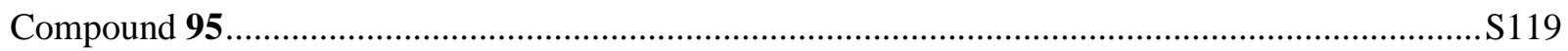

Compound 96

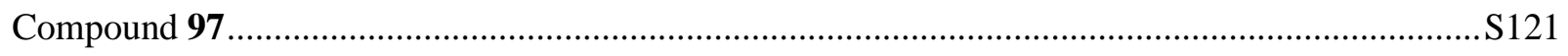

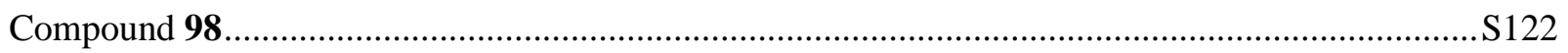

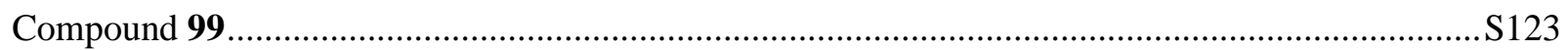

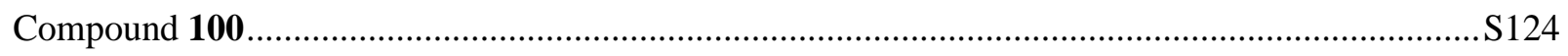

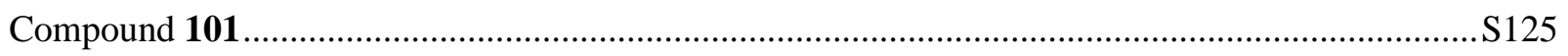

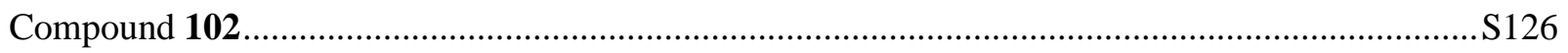

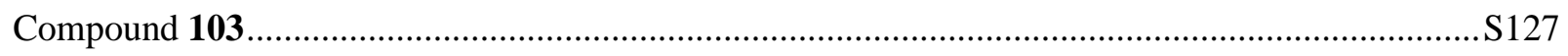

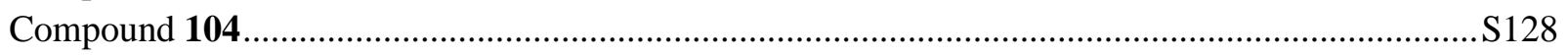

Compound 105

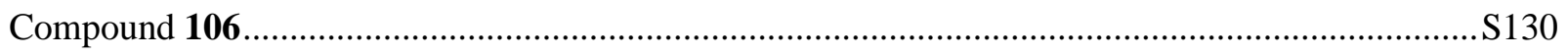

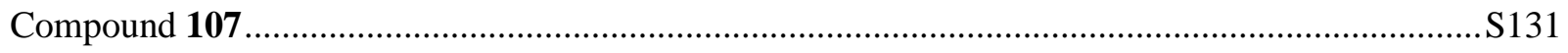

Compound 108

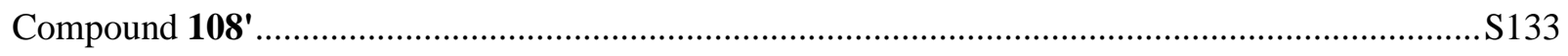

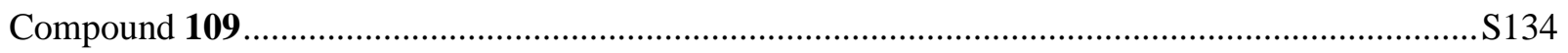

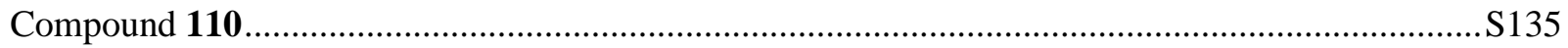

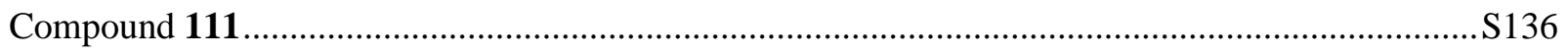

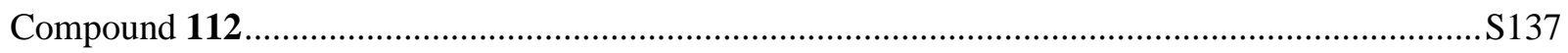

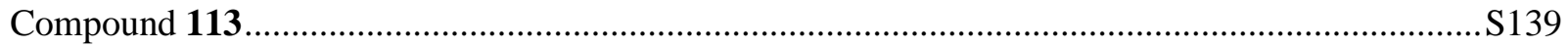

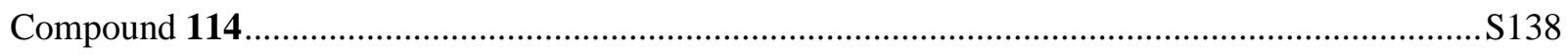

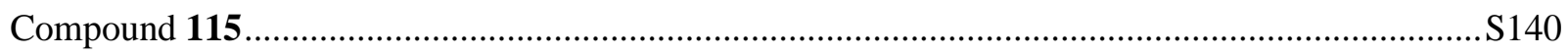

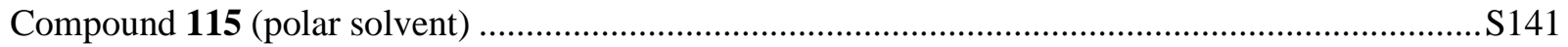

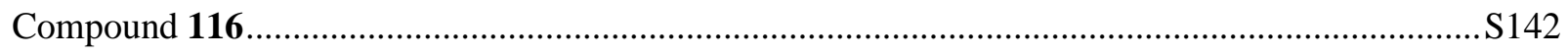

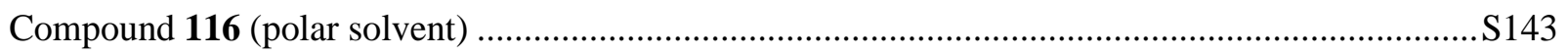

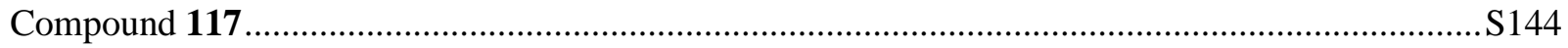

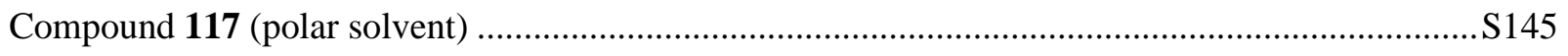

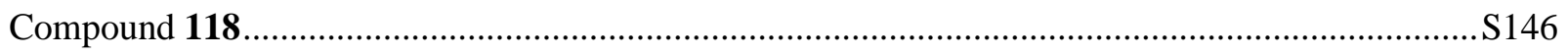

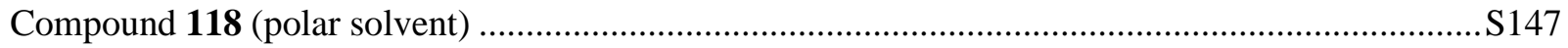

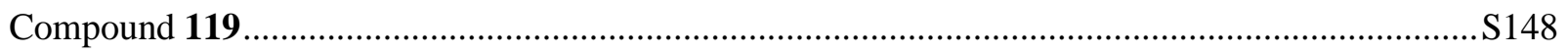

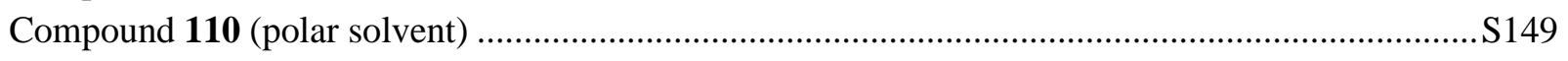

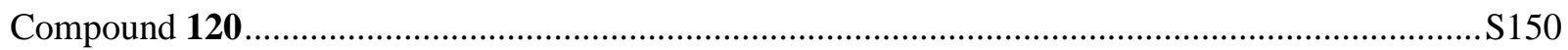

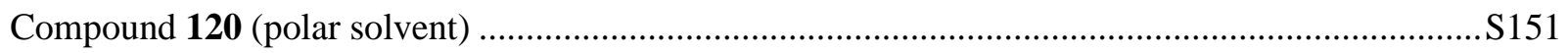




\section{General Methods}

CoGEF calculations were performed using Spartan '18 Parallel Suite according to previously reported methods. ${ }^{1}$ Chemical structures were composed in ChemDraw, saved as .mol files, and then imported into Spartan. Structures were truncated to include tethers that accurately reflect the structure of the molecules used in the experimental studies. Ground state energies were calculated using DFT at the B3LYP/6-31G* level of theory in vacuum, unless specified otherwise. For the three mechanophores in the heterolytic category, CoGEF calculations were also performed using a polarizable continuum model (dielectric constant of 37) to simulate a polar solvent. Starting from the equilibrium geometry of the unconstrained molecule (relative energy $=0 \mathrm{~kJ} / \mathrm{mol}$ ), the distance between the terminal anchor atoms of the truncated structure was increased in increments of 0.05 $\AA$ and the energy was minimized at each step. This operation was carried out automatically using the Energy Profile calculation in Spartan. Calculations were run until a chemical transformation was predicted to occur, as evidenced by the rupture and reorganization of one or more covalent bonds. In some cases, an initial equilibrium conformer calculation was performed using Molecular Mechanics (MMFF) before performing the steps outlined above. The maximum number of geometry optimization cycles was increased beyond the default value using the GEOMETRYCYCLE option to ensure convergence at each step in the CoGEF profile.

Determination of $\boldsymbol{F}_{\text {max }}$. The maximum force predicted for each mechanochemical transformation was calculated from the slope between contiguous points in the energy-displacement curve. In most cases, $F_{\max }$ coincides with the displacement immediately prior to a discontinuity in the relative energy profile. The value of $F_{\max }$ is thus calculated from the slope between the two data points preceding the abrupt attenuation in energy. More rarely, a continuous change in energy is observed that approaches an apparent plateau value at long displacements. In these cases, $F_{\max }$ occurs at the inflection point in the CoGEF curve. The value of the slope is divided by the Avogadro constant and adjusted to provide force in units of $\mathrm{nJ} / \mathrm{m}(\mathrm{nN})$.

Determination of $\boldsymbol{E}_{\max }$. The maximum energy relative to the energy of the unconstrained molecule at equilibrium is reported as $E_{\max }$. The value of $E_{\max }$ is determined from the CoGEF curve at the displacement corresponding to $F_{\max }$. Typically, this means that $E_{\max }$ represents the highest relative energy on the CoGEF curve; however, for instances in which the CoGEF profile exhibits a sigmoidal shape and/or a discontinuity is absent, $E_{\max }$ corresponds to the relative energy at the inflection point.

Determination of Force-Bond Angle. Force-bond angles were calculated according to the previously described method using structural models from CoGEF calculations at the displacement corresponding to $F_{\max }{ }^{2}$ The external force vector was approximated using the coordinates of the two terminal atoms that define the distance constraint in the CoGEF calculation.

(1) (a) Beyer, M. K. The Mechanical Strength of a Covalent Bond Calculated by Density Functional Theory. J.

Chem. Phys. 2000, 112, 7307-7312. (b) Kryger, M. J.; Munaretto, A. M.; Moore, J. S. Structure-Mechanochemical Activity Relationships for Cyclobutane Mechanophores. J. Am. Chem. Soc. 2011, 133, 18992-18998.

(2) Robb, M. J.; Kim, T. A.; Halmes, A. J.; White, S. R.; Sottos, N. R.; Moore, J. S. Regioisomer-Specific Mechanochromism of Naphthopyran in Polymeric Materials. J. Am. Chem. Soc. 2016, 138, 12328-12331. 


\section{Supplementary Figures}

A

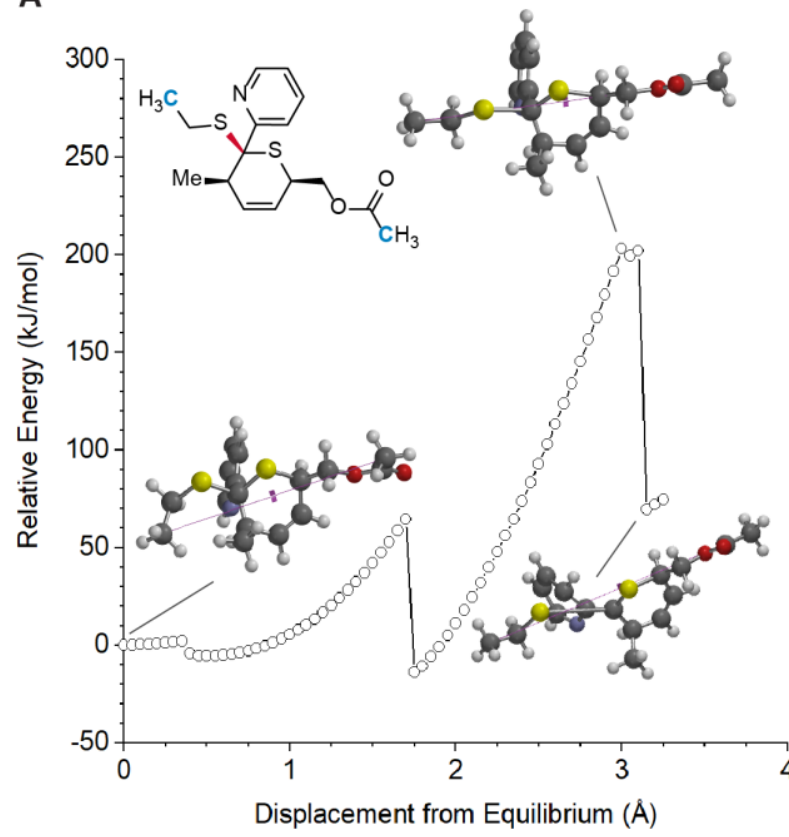

C

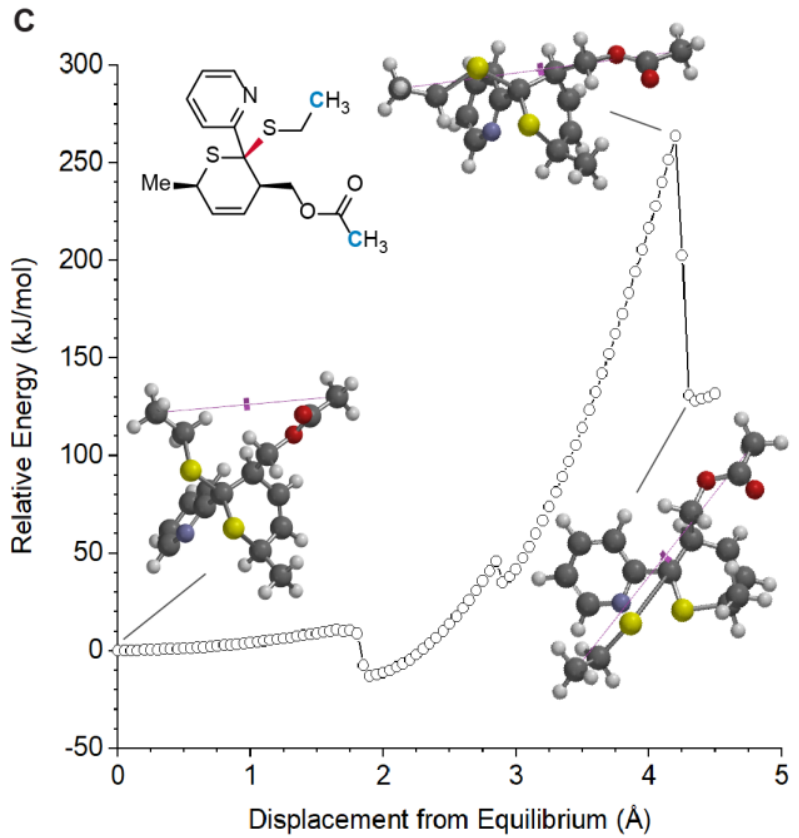

B

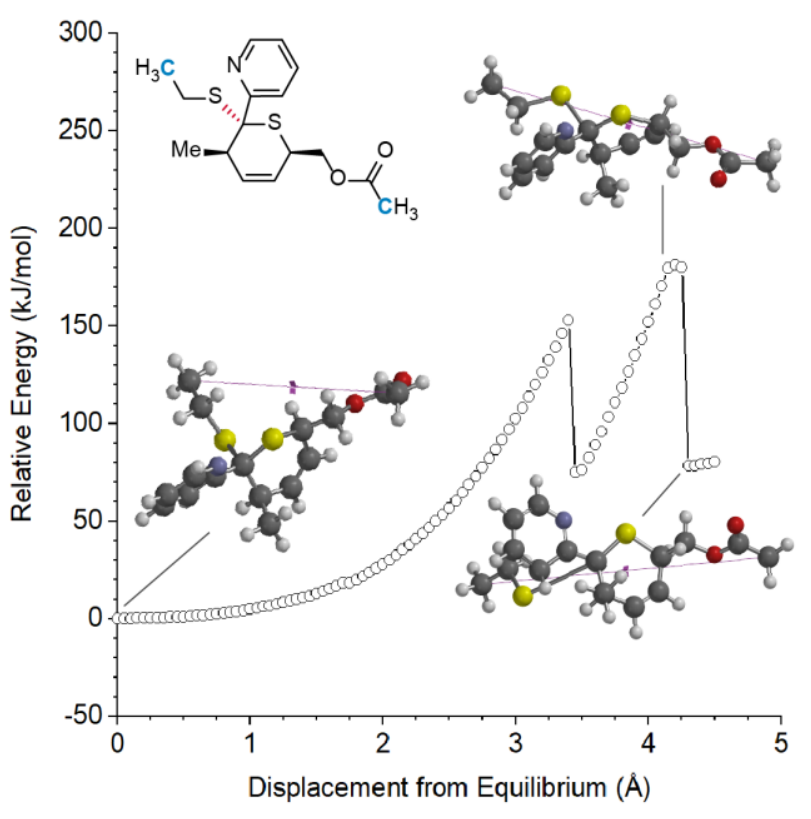

D

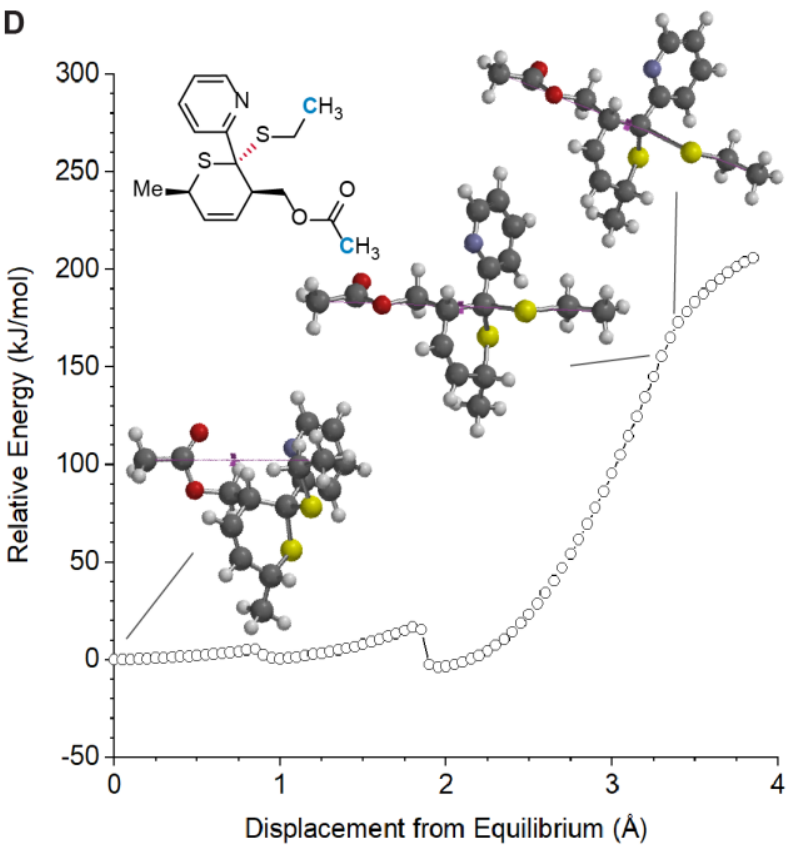

Figure S1. CoGEF results for four possible isomers of a hetero-Diels-Alder adduct corresponding to the reactive subunits of reported mechanophore 36 . All isomers are predicted to undergo $\mathrm{C}-\mathrm{S}$ bond scission rather than the formal retro-[4+2] cycloaddition reaction. 
A

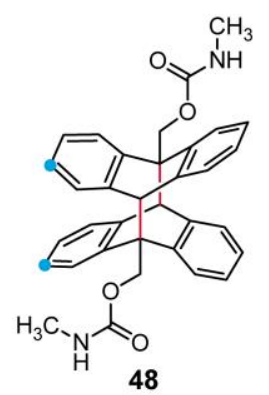<smiles>CNC(=O)OCc1cccc2ccccc12</smiles>

B

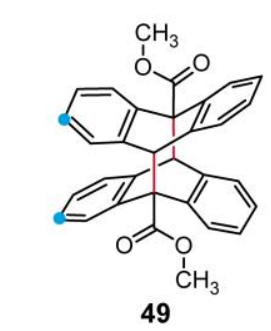<smiles>COC(=O)c1c2ccccc2cc2ccccc12</smiles>

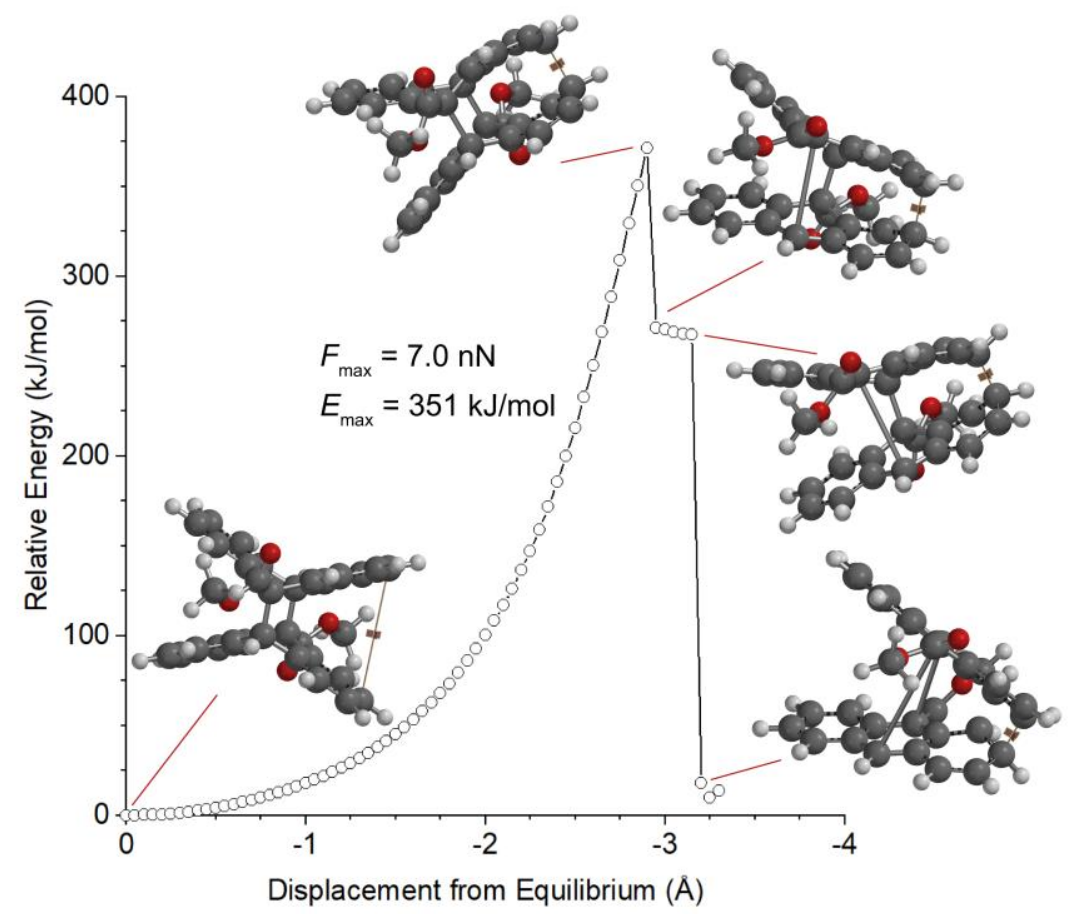

Figure S2. CoGEF calculations performed in an alternative compression mode for head-to-tail anthracene dimer mechanophores (A) 48 and (B) 49. The distance between carbon atoms labeled with a blue dot was decreased incrementally starting from the force-free equilibrium geometry. At each step, the geometry was optimized at the B3LYP/6-31G* level of DFT. Both molecules are predicted to undergo a formal retro-[4+4] cycloaddition reaction upon simulated compression. The transformation proceeds through an apparent stepwise pathway suggesting an intermediate with diradicaloid character. 
A
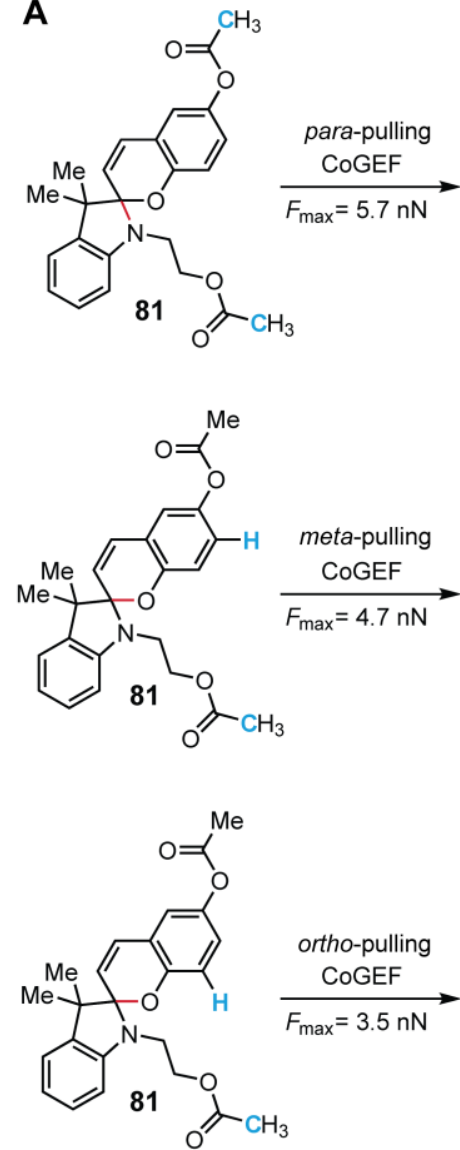
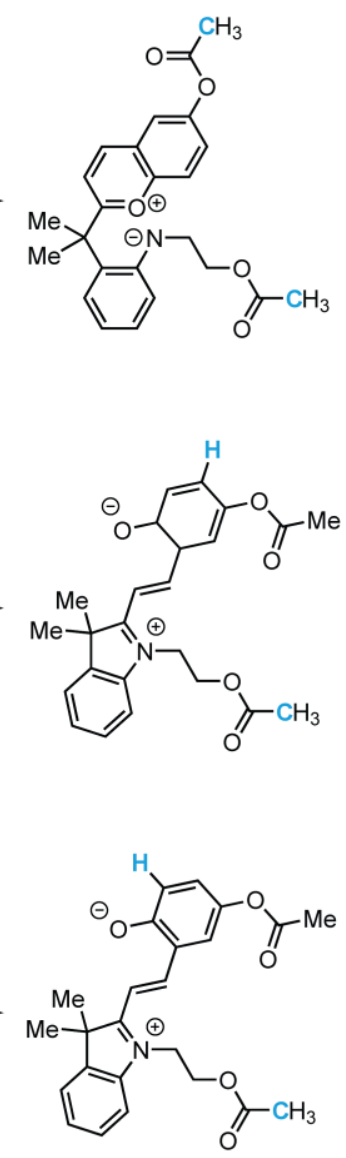

B

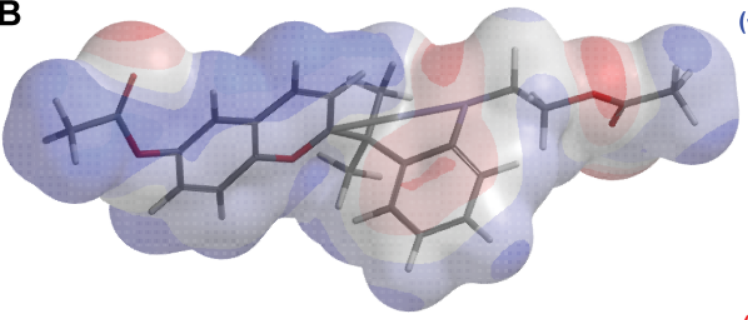

$(+)+125$

C

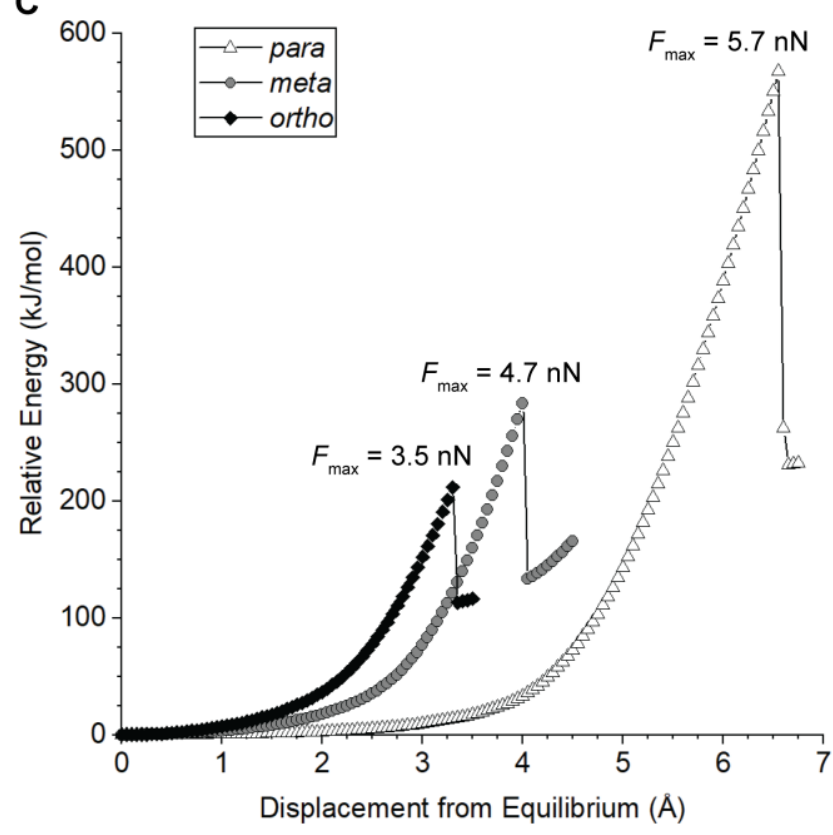

D
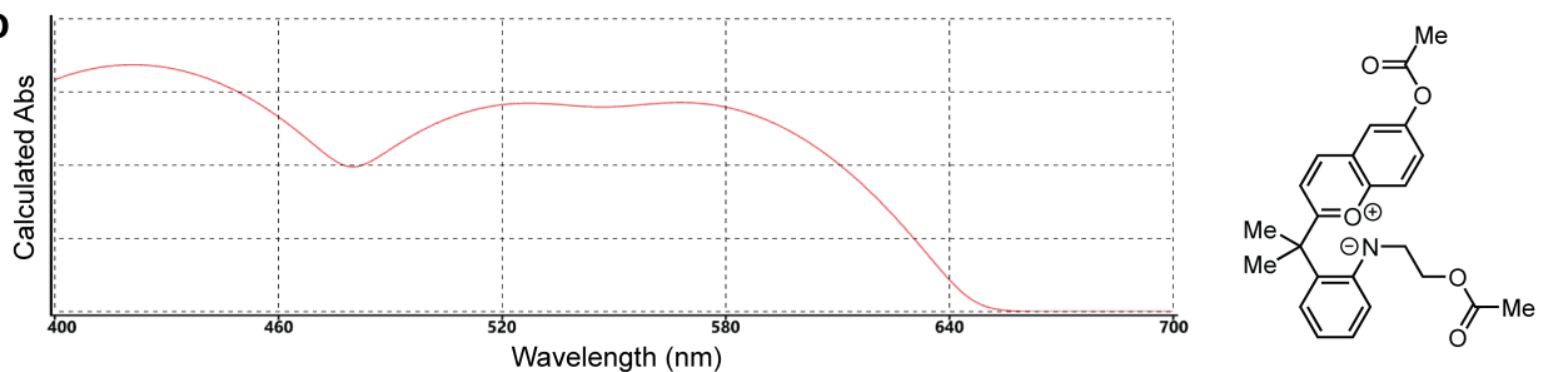

E
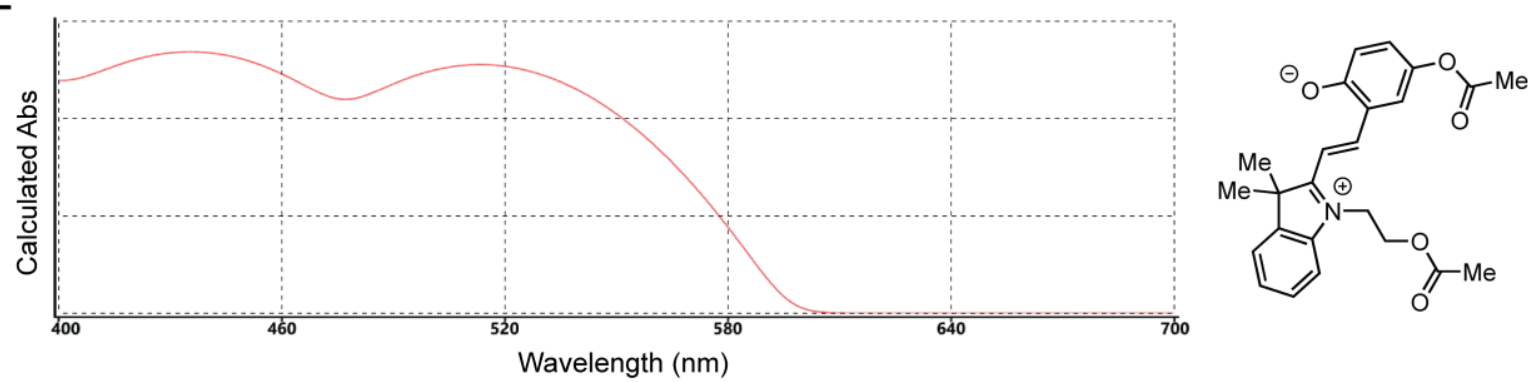

Figure S3. Investigation of regiochemical effects on the predicted mechanochemical reactivity of spiropyran 81. (A) Changing the pulling position results in the anticipated scission of the $\mathrm{C}-\mathrm{O}$ pyran bond leading to formation of the merocyanine. (B) Electrostatic potential map of the product predicted by CoGEF (parapulling) indicating heterolytic fragmentation of the $\mathrm{C}-\mathrm{N}$ bond. (C) CoGEF profiles associated with the schemes in panel A. (D, E) Visible absorption spectra calculated at the B3LYP/6-31G* level of TD-DFT for the product resulting from $\mathrm{C}-\mathrm{N}$ bond scission, and the expected merocyanine species. 
A

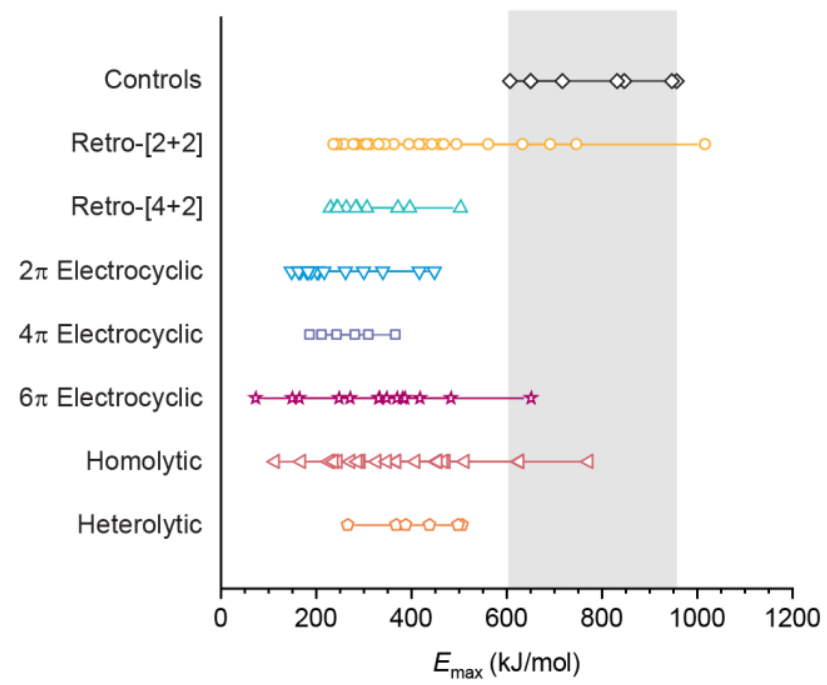

B

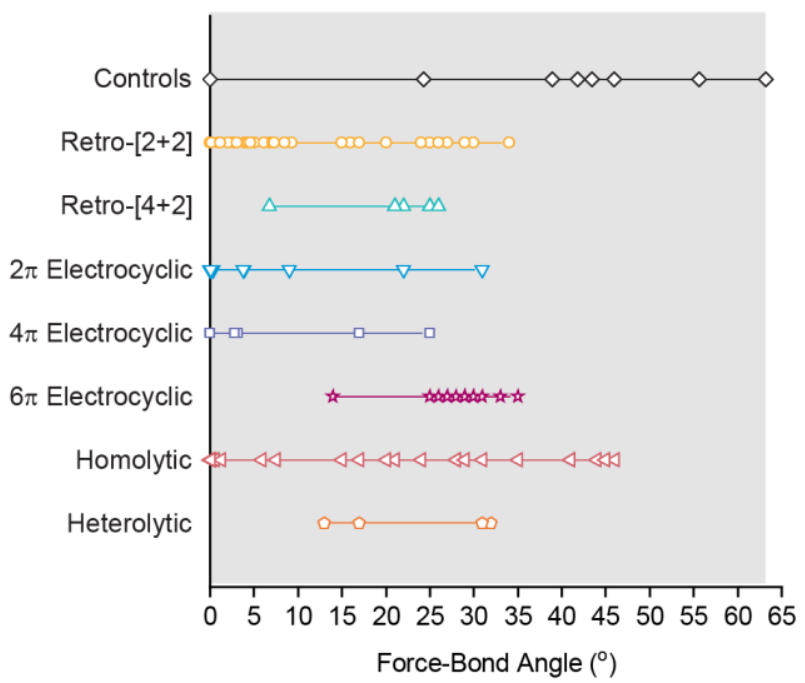

Figure S4. Summary of $(A) E_{\max }$ values and $(B)$ force-bond angles determined using the CoGEF method for each mechanochemical reaction class. The CoGEF results for control structures are universally indistinguishable from the mechanophores when alternative quantitative metrics $E_{\max }$ and force-bond angle are compared, indicating that these metrics are poor predictors of mechanochemical activity. Data from calculations that are inconsistent with reported experimentally determined reactivity are excluded.

A

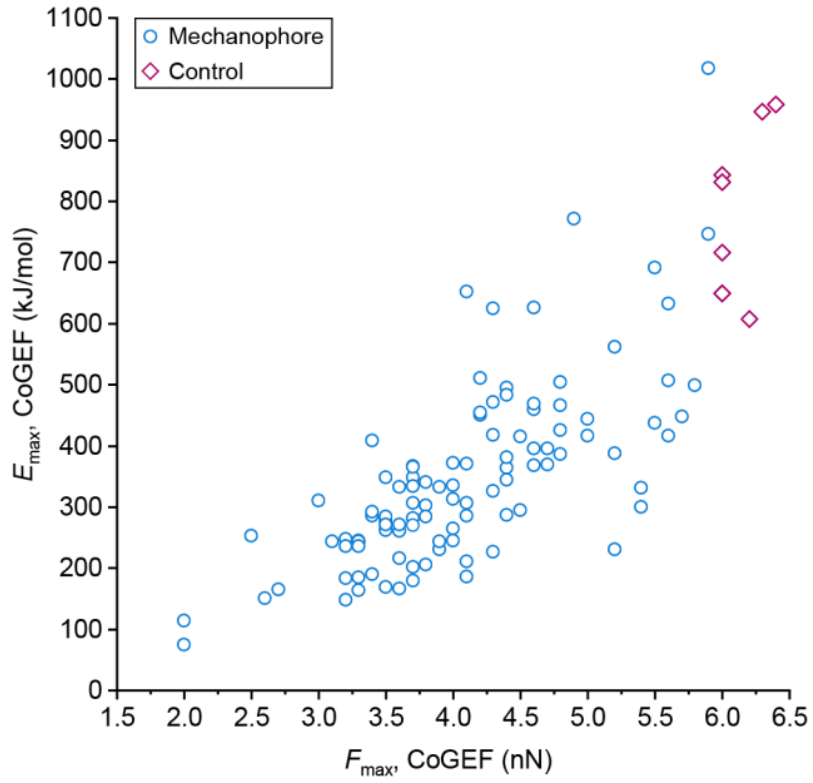

B

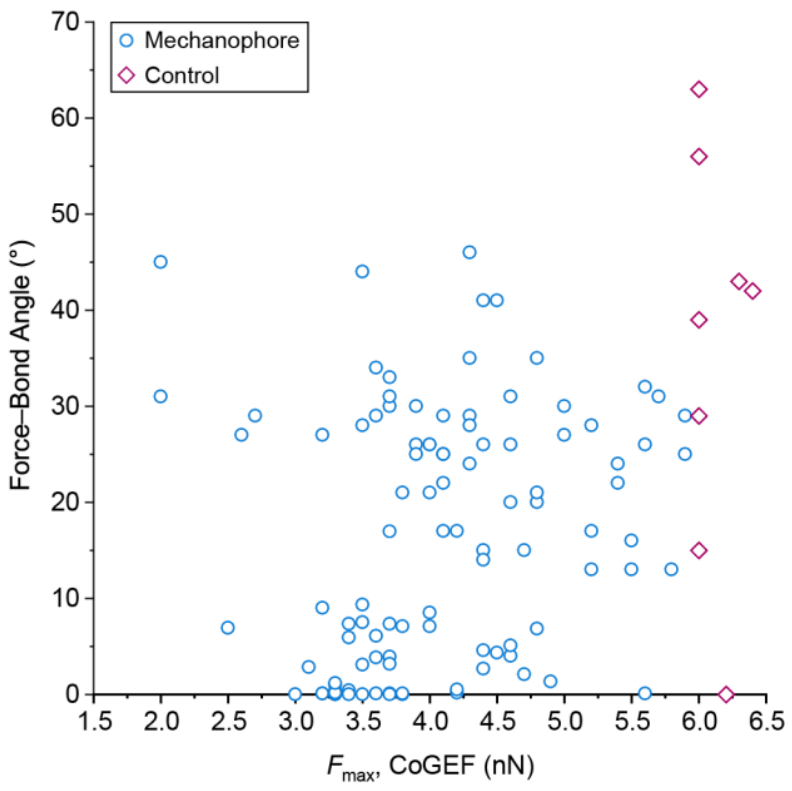

Figure S5. Relationship between calculated values of $(A) E_{\max }$ and $(B)$ force-bond angle with the calculated values of $F_{\max }$ determined with the CoGEF method at the B3LYP/6-31G* level of density functional theory. There is a positive correlation between values of $E_{\max }$ and $F_{\max }$, while there is no apparent correlation between force-bond angle and values of $F_{\text {max }}$. 

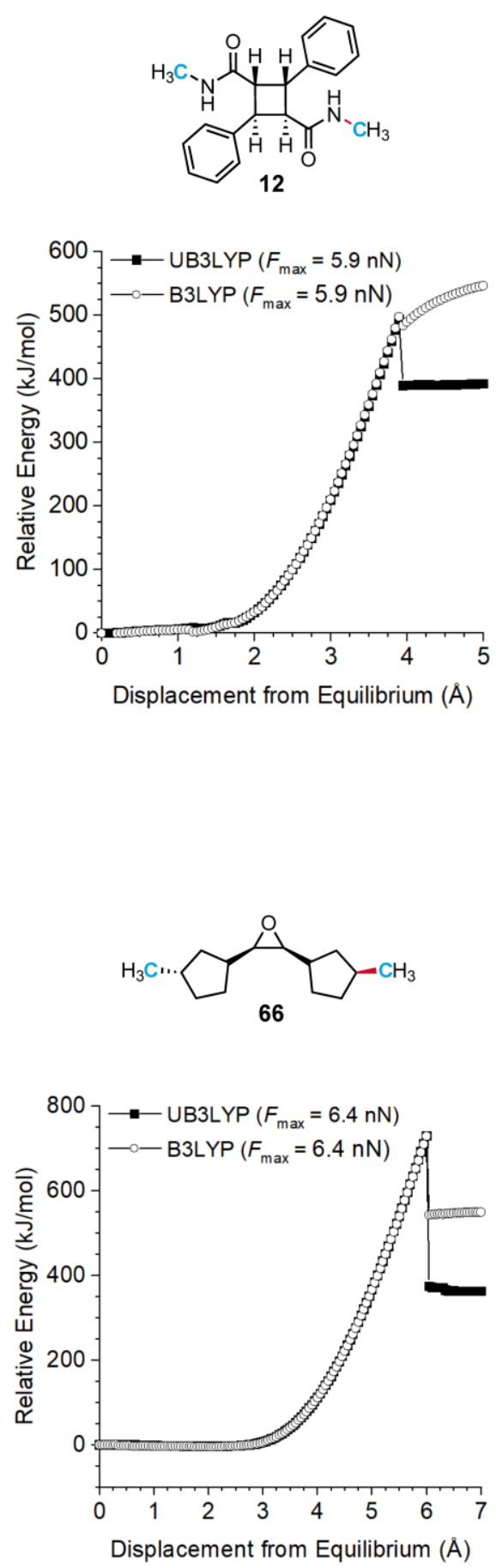
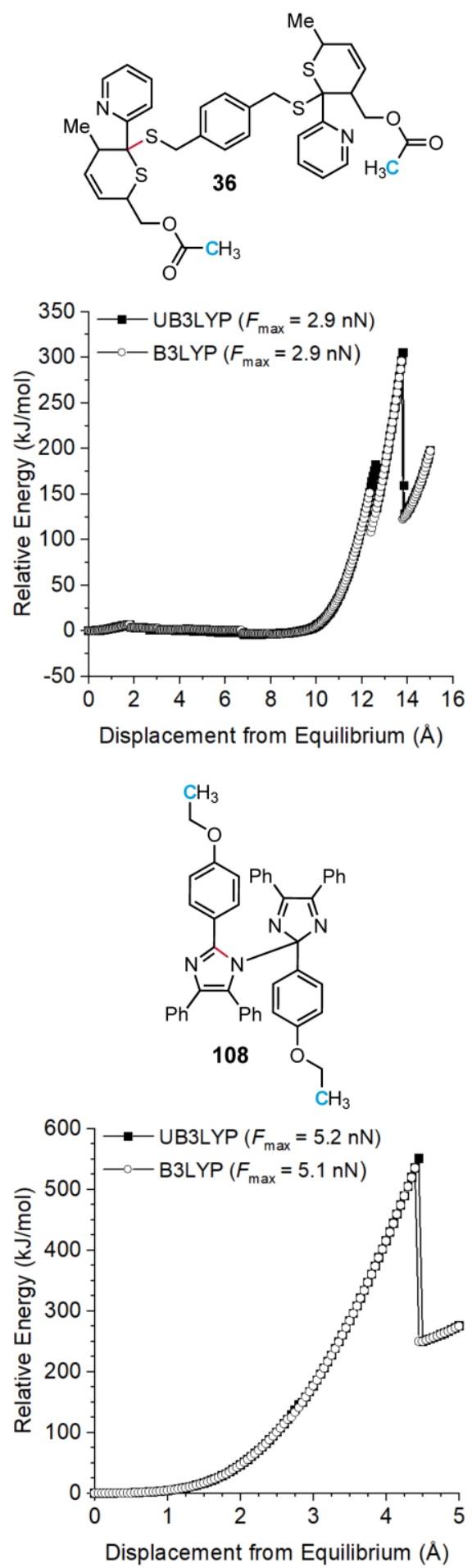
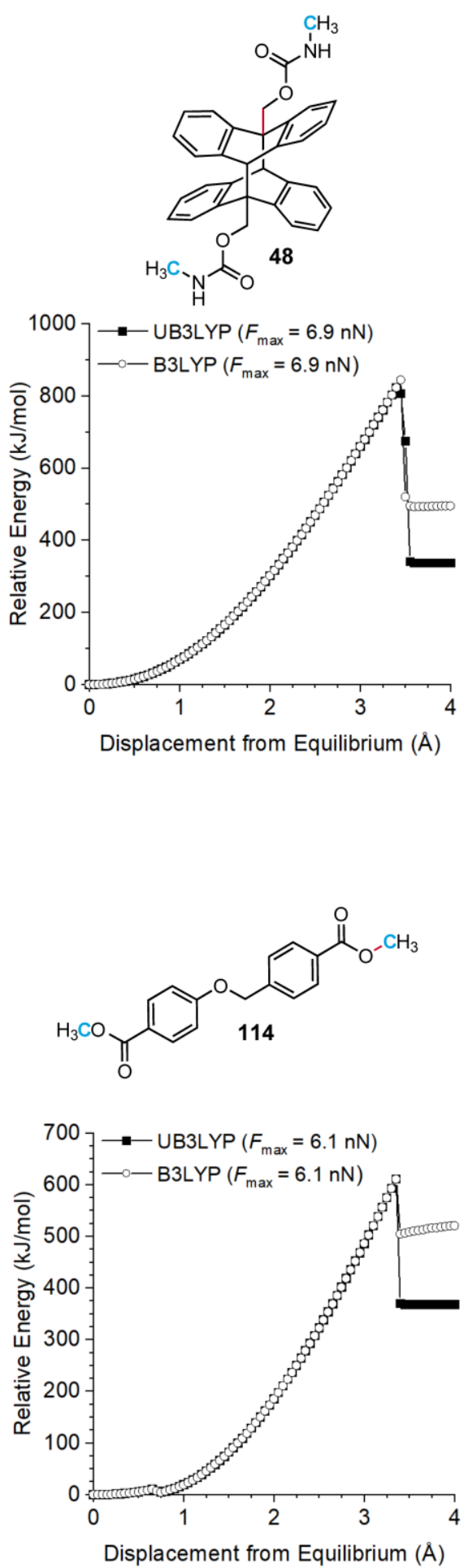

Figure S6. CoGEF calculations performed using unrestricted DFT (UB3LYP/6-31G*) on representative mechanophores for which CoGEF calculations at the B3LYP/6-31G* level of DFT predict reactions that are inconsistent with the reported experimental behavior. Use of the UB3LYP functional has minimal influence on the results of the CoGEF simulations. The same chemical transformations are predicted in each case. 

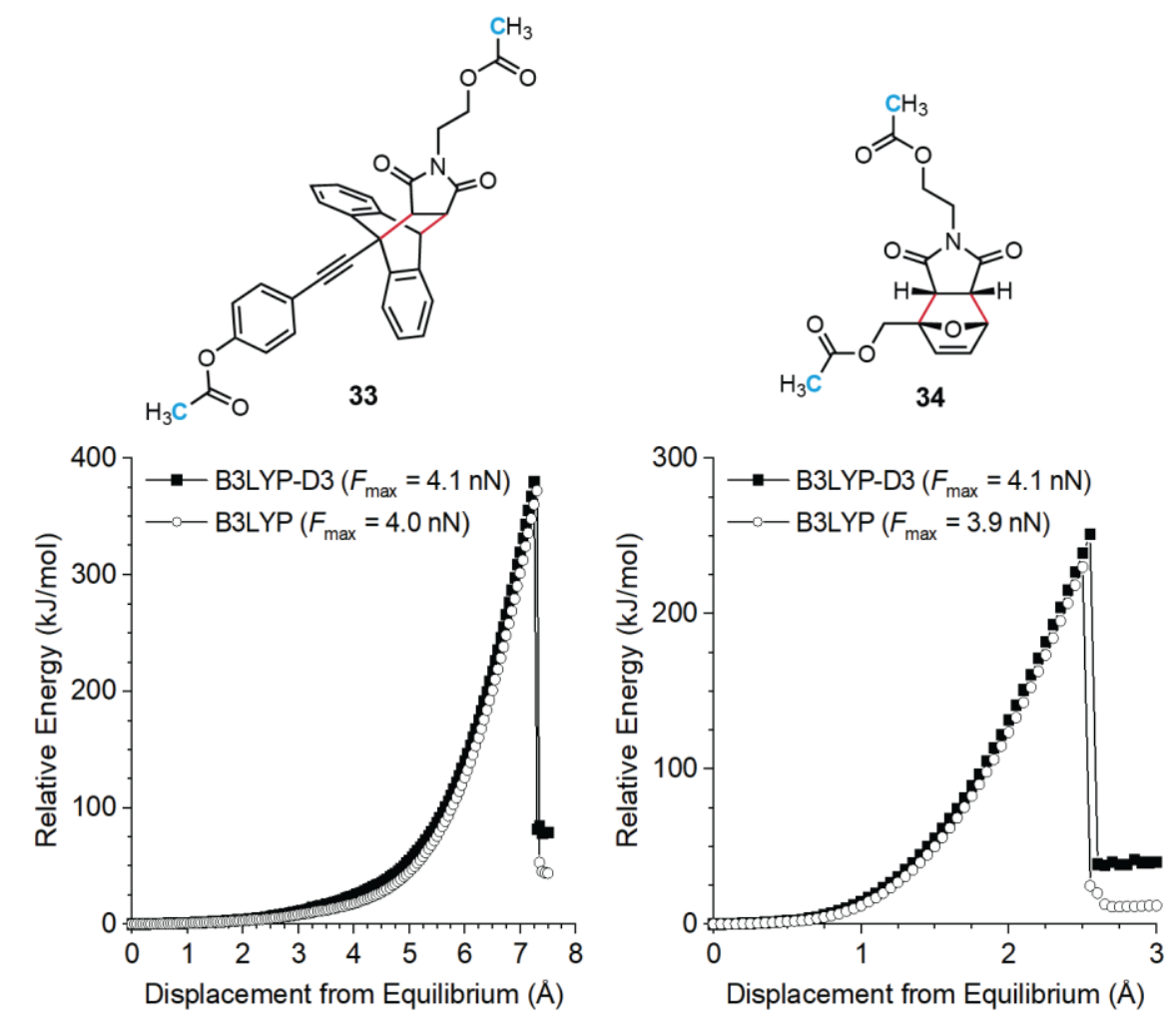

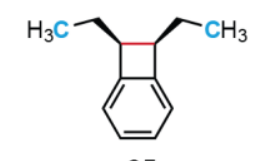

65

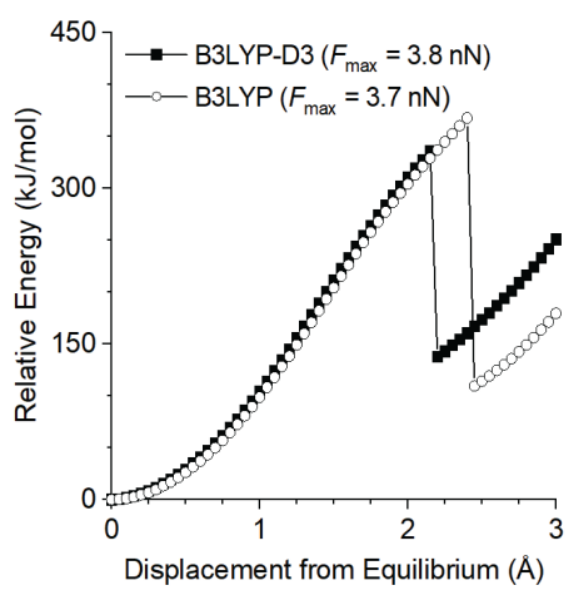

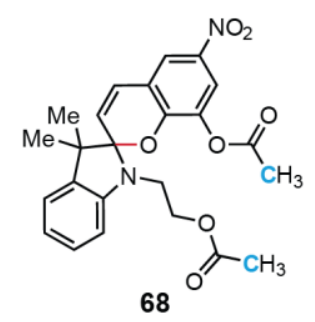

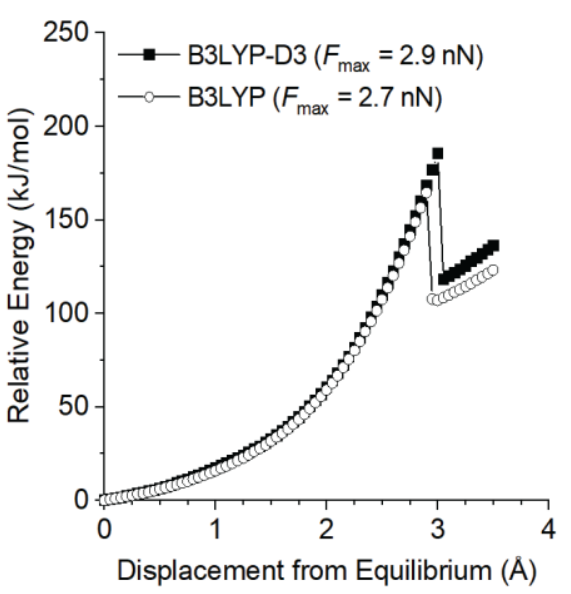

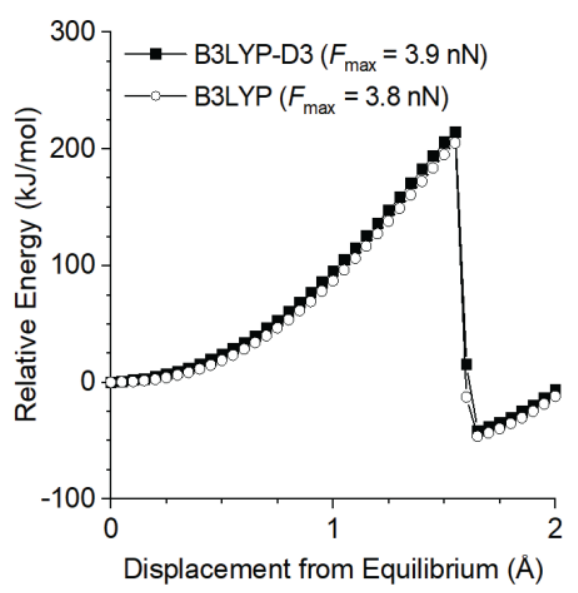
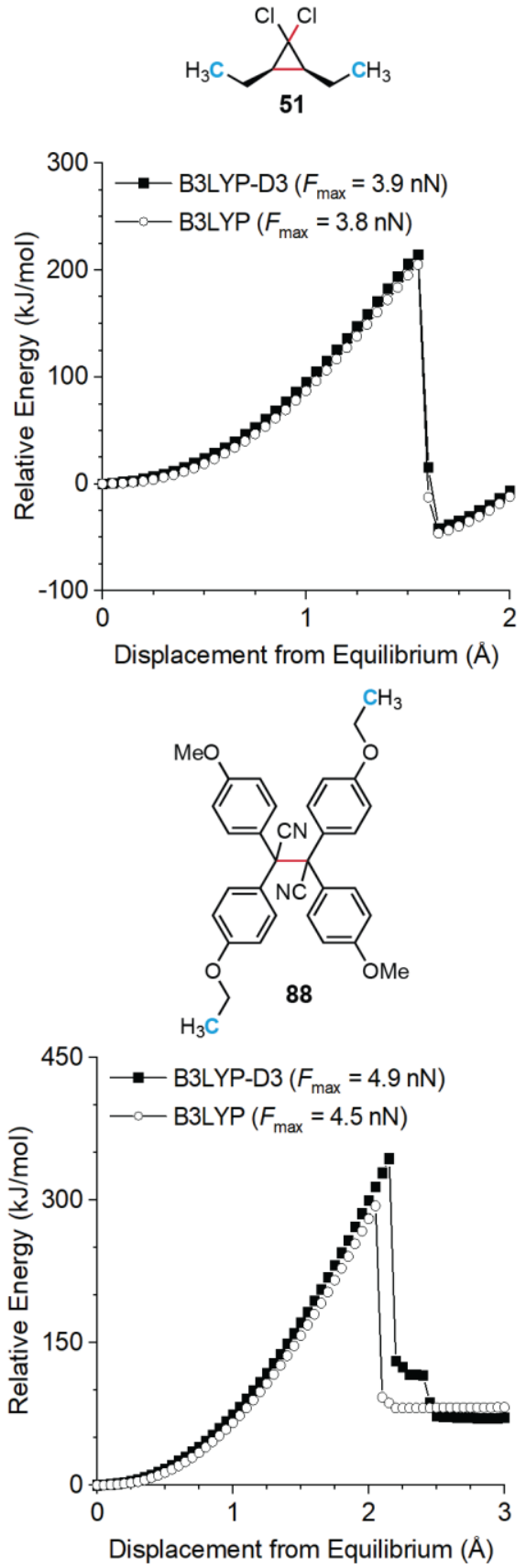

Figure S7. Comparison of CoGEF calculations performed on representative mechanophores at the B3LYP/6-31G* level of DFT and using a dispersion-corrected functional (B3LYP-D3/6-31G*). Use of the dispersion-corrected B3LYP-D3 functional has minimal influence on the results of the CoGEF simulations. The same chemical transformations are predicted in each case. 


\section{Summaries of Individual CoGEF Calculations}

A summary of the results of each individual CoGEF calculation are presented on the pages below. All calculations were performed using DFT at the B3LYP/6-31G* level of theory in vacuum, unless specified otherwise. A reaction scheme depicts the structure of the truncated molecule and the product(s) predicted from the CoGEF calculation. The atoms colored blue indicate the anchor positions (i.e., pulling points) for defining the distance constraint and the bonds that are predicted to cleave are colored red. Representative images of computed structures at critical points in the CoGEF profile are included that depict the force-free equilibrium geometry as well as the structure(s) immediately before and after bond cleavage events. The length of the distance constraint is included below each computed structure and the corresponding positions on the CoGEF curve are denoted. Electrostatic potential maps are included for the products predicted by CoGEF calculations in the heterolytic category. The calculated values of $F_{\max }, E_{\max }$, and forcebond angle are tabulated for each calculation. Note that the former bonds persist as artifacts in Spartan after a reaction is predicted to occur. For references to the primary literature describing the experimental reactivity of each compound, refer to the tables in the main text.
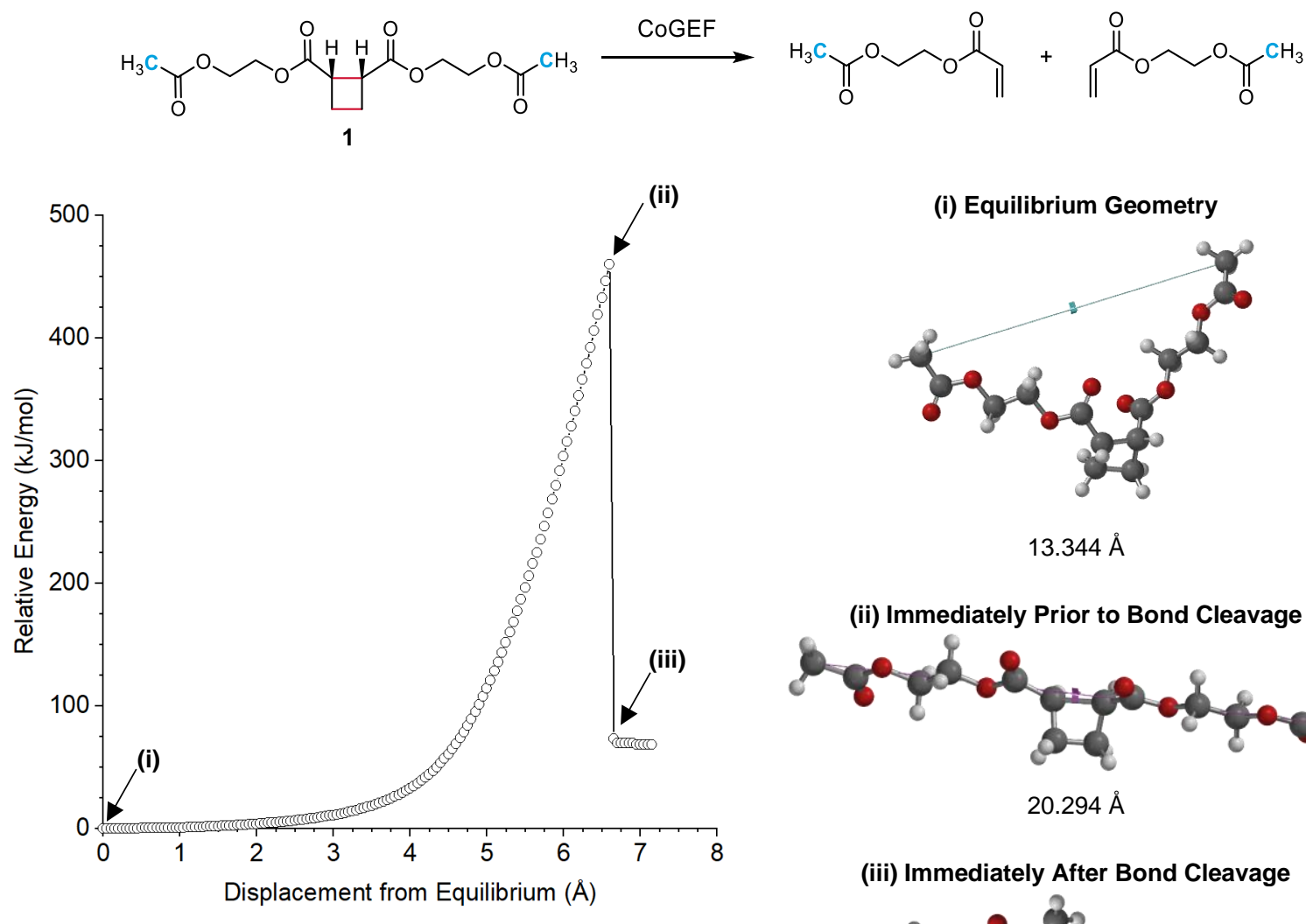

(i) Equilibrium Geometry

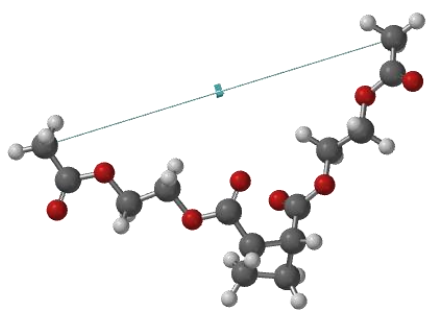

$13.344 \AA$

(ii) Immediately Prior to Bond Cleavage

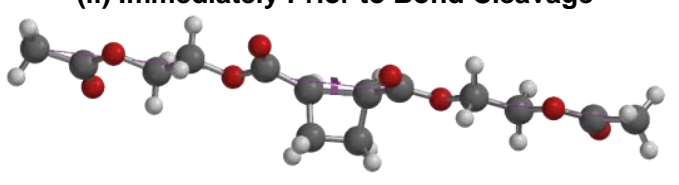

$20.294 \AA$

(iii) Immediately After Bond Cleavage
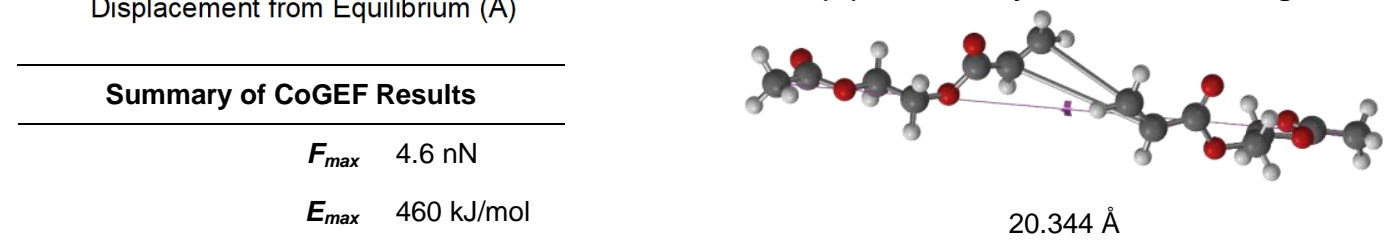

$20.344 \AA$

Force-Bond Angle $4^{\circ}$ 


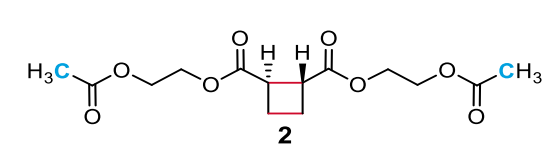

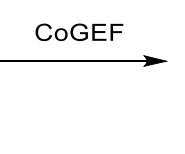

(ii)
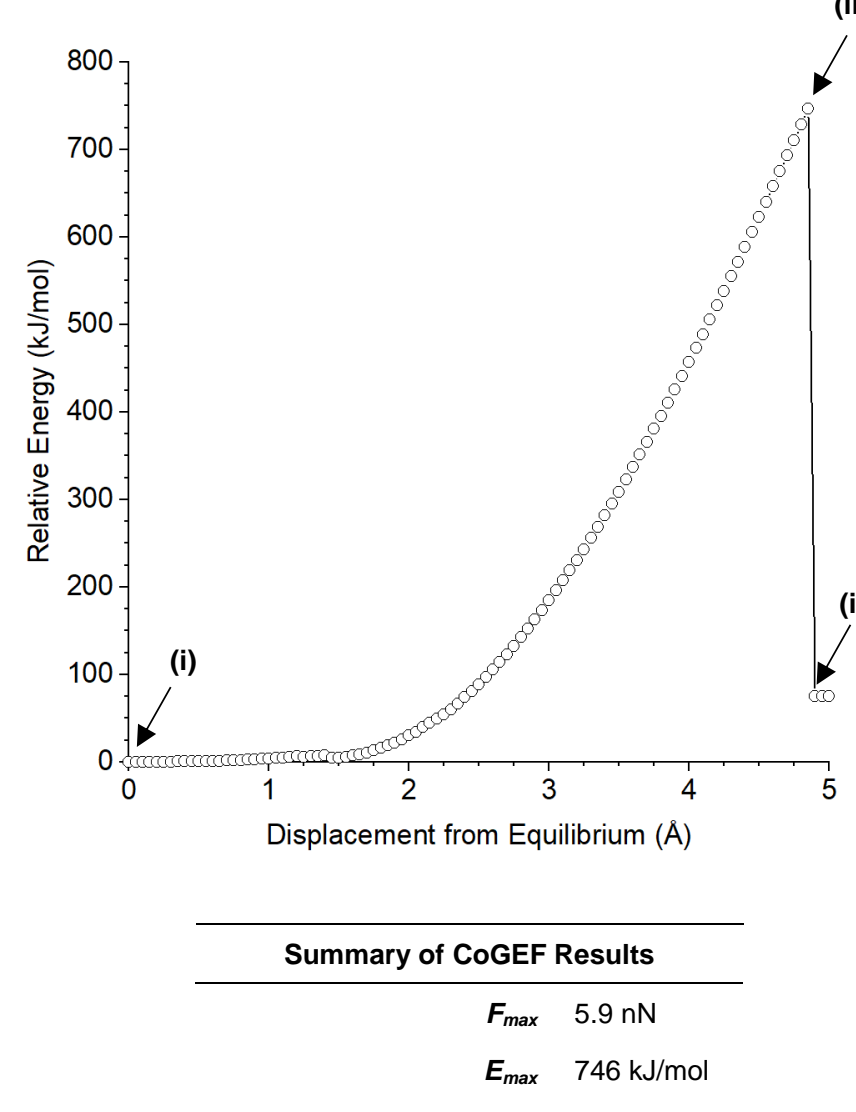

Force-Bond Angle $25^{\circ}$<smiles>C=IC(=O)OCCOC(=O)C=[CH+]C(=O)OCCOC(C)=O</smiles>

(i) Equilibrium Geometry

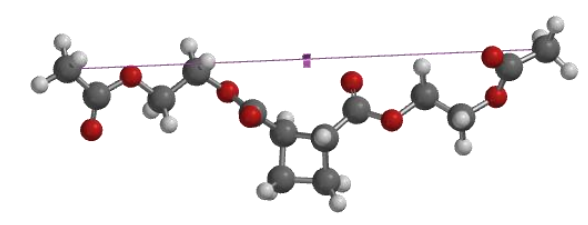

$16.382 \AA$

(ii) Immediately Prior to Bond Cleavage

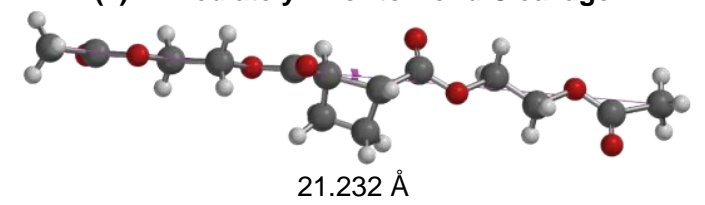

(iii) Immediately After Bond Cleavage

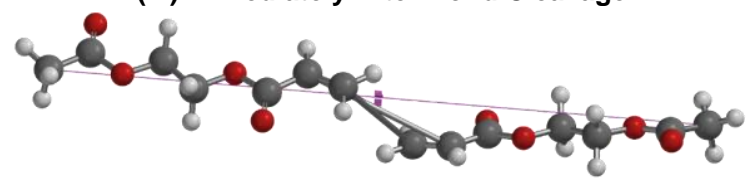

$21.282 \AA$ 
<smiles>CC(=O)OCCOC(=O)[C@H]1CC[C@@]1(C#N)[C@@H](C)C(=O)OCCOC(C)=O</smiles>
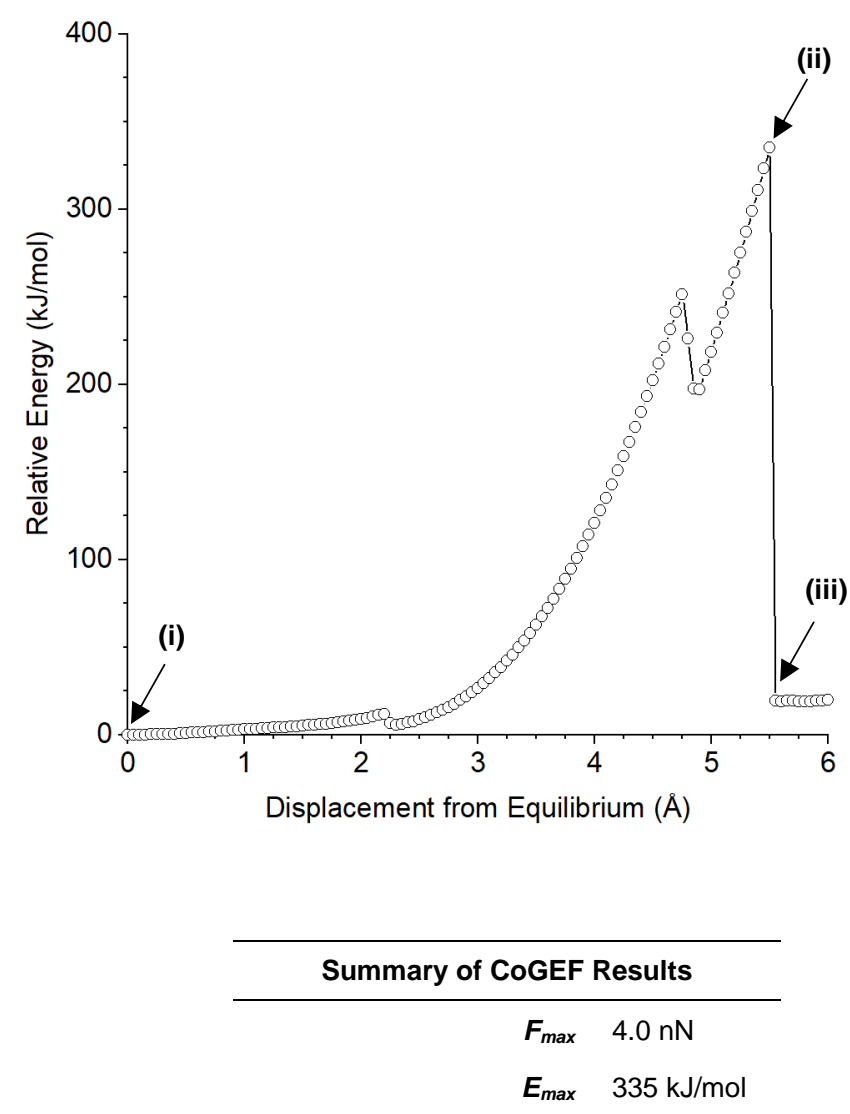

Force-Bond Angle $7.1^{\circ}$<smiles>C=CC(=O)OCCOC(C)=O</smiles>

(i) Equilibrium Geometry

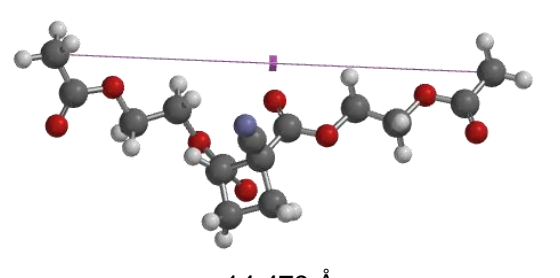

$14.479 \AA$

(ii) Immediately Prior to Bond Cleavage

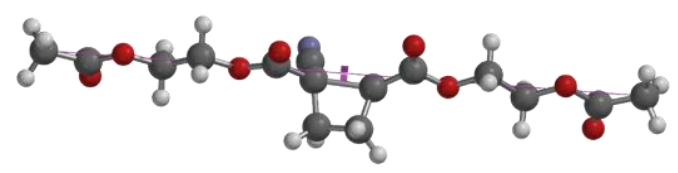

$19.979 \AA$

(iii) Immediately After Bond Cleavage

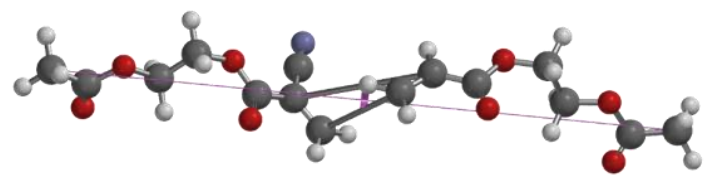

$20.029 \AA$ 

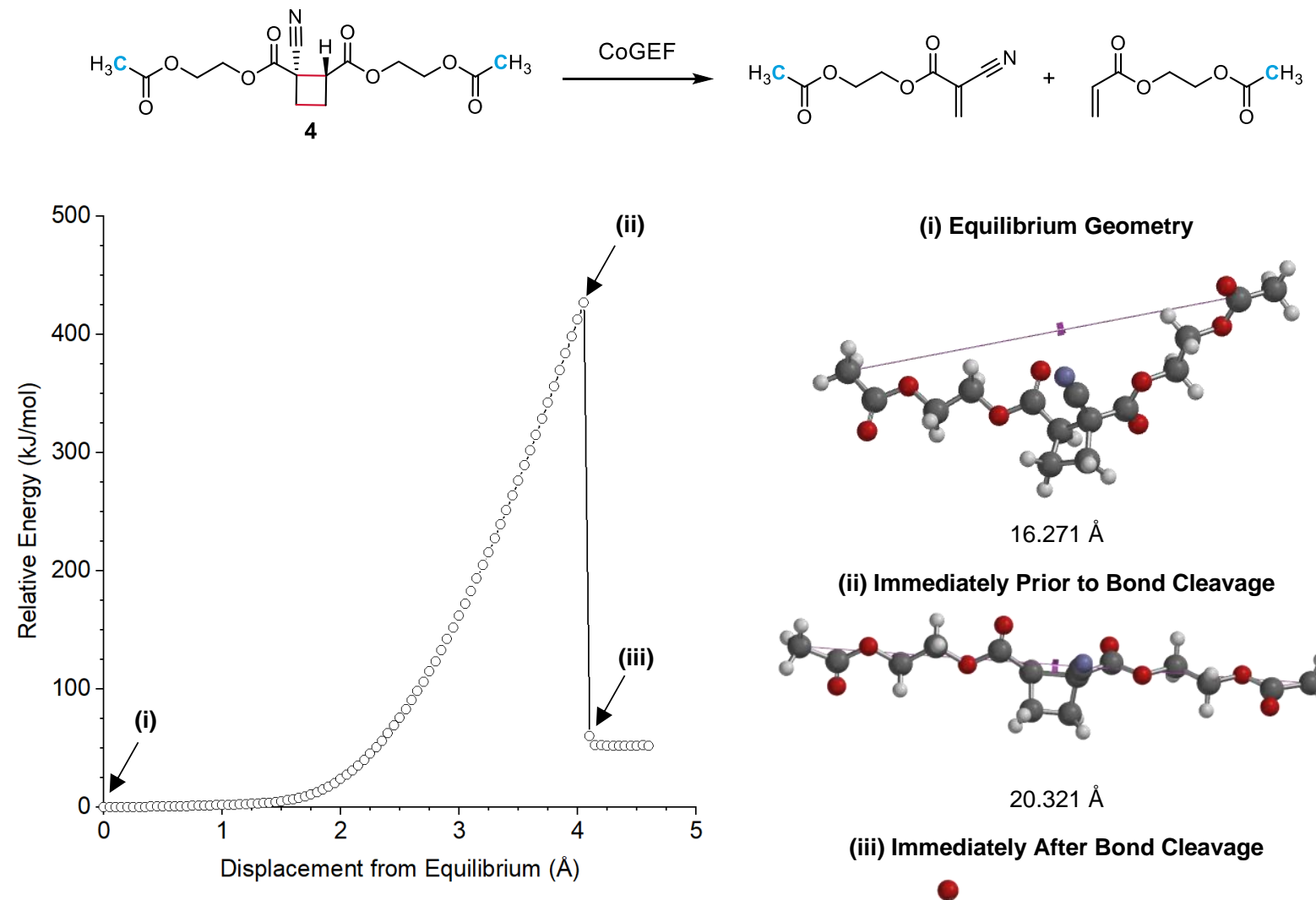

$16.271 \AA$

(ii) Immediately Prior to Bond Cleavage

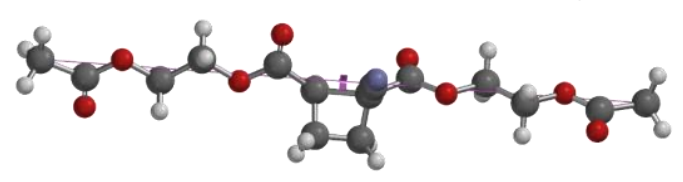

$20.321 \AA$

(iii) Immediately After Bond Cleavage

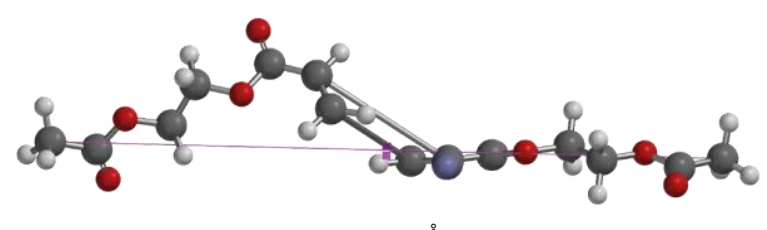

$20.371 \AA$

Force-Bond Angle $20^{\circ}$ 


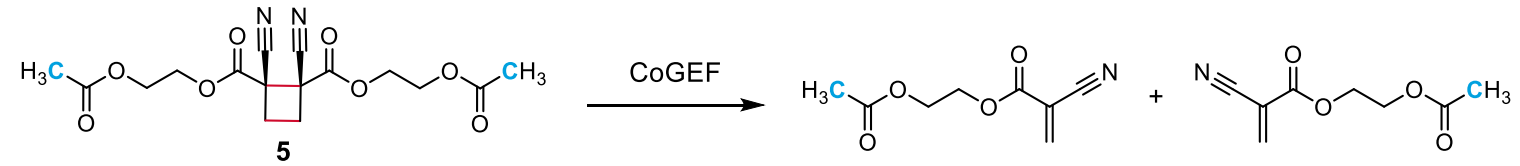

(ii)

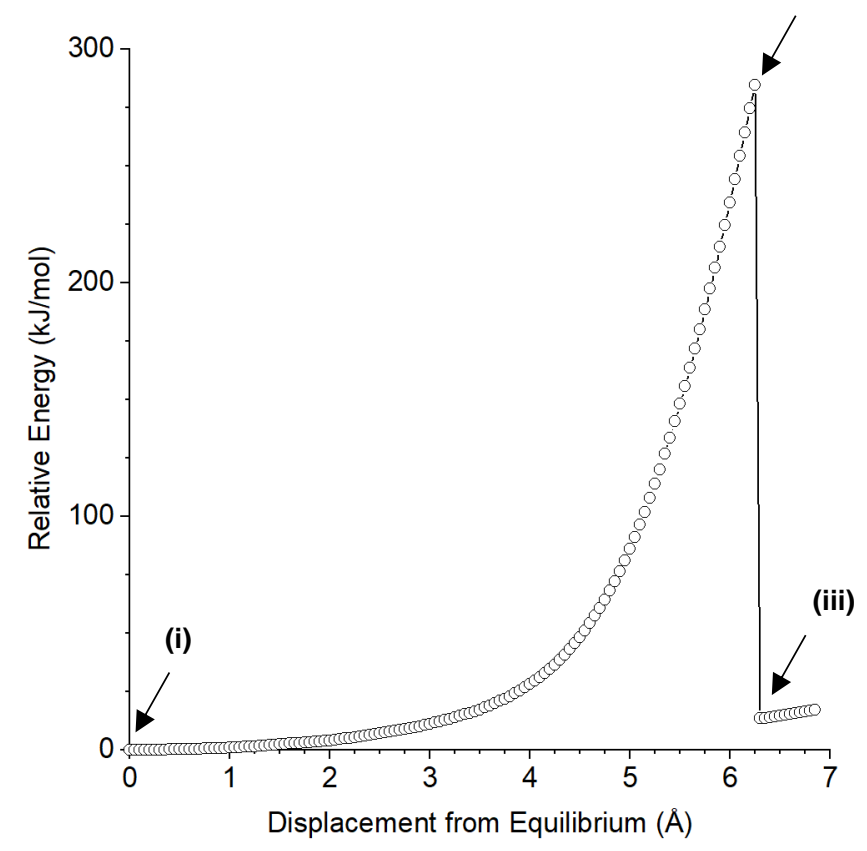

(i) Equilibrium Geometry

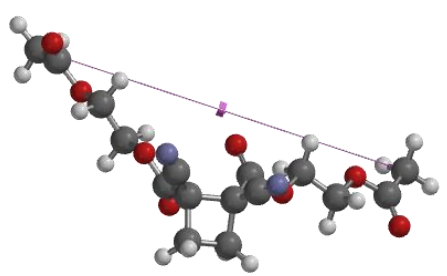

$13.303 \AA$

(ii) Immediately Prior to Bond Cleavage

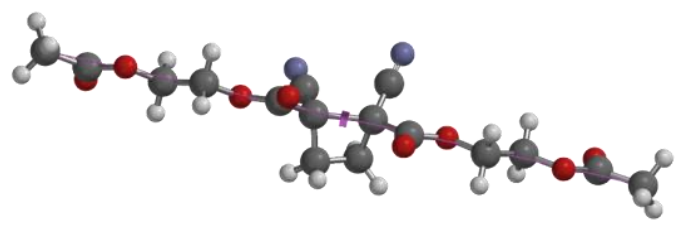

$19.553 \AA$

(iii) Immediately After Bond Cleavage

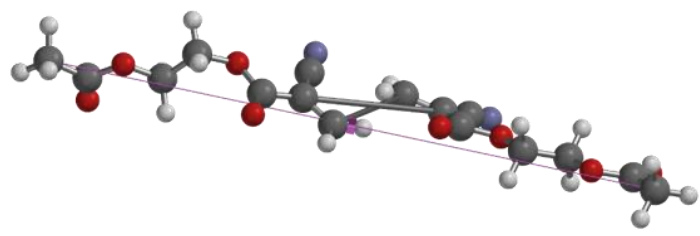

$19.603 \AA$

Force-Bond angle $7.3^{\circ}$ 
<smiles>C=C(C#N)C(=O)OCCOC(=O)C(=C)C#[N+]</smiles>

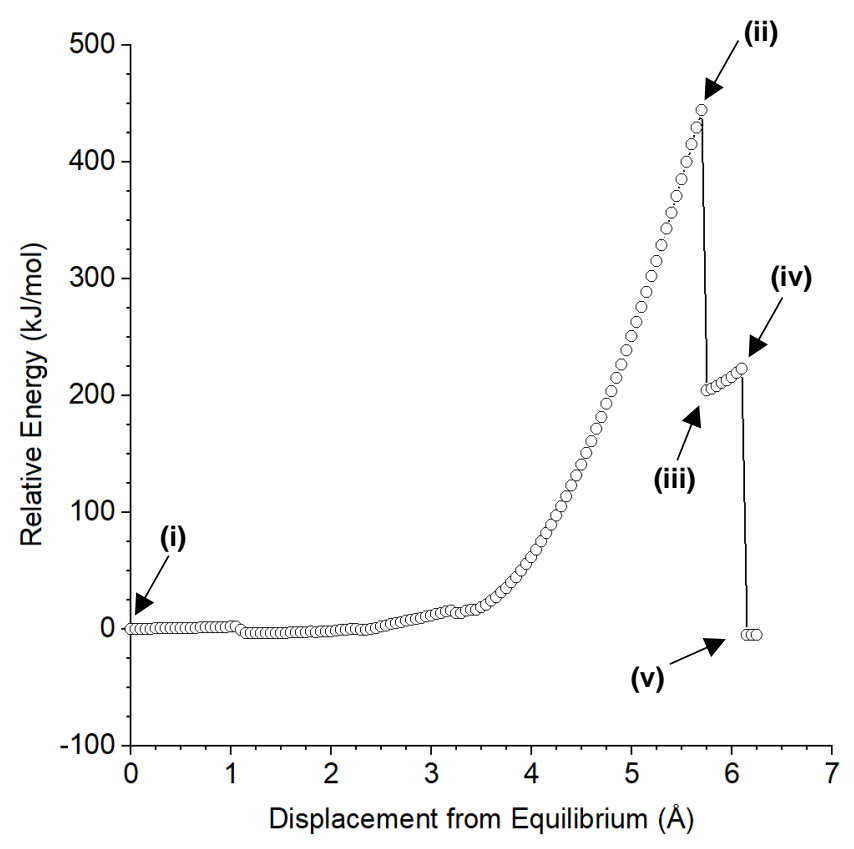

Summary of CoGEF Results

$$
\begin{array}{ll}
\boldsymbol{F}_{\text {max }} & 5.0 \mathrm{nN} \\
\boldsymbol{E}_{\max } & 444 \mathrm{~kJ} / \mathrm{mol}
\end{array}
$$

Force-Bond Angle $27^{\circ}$ (i) Equilibrium Geometry

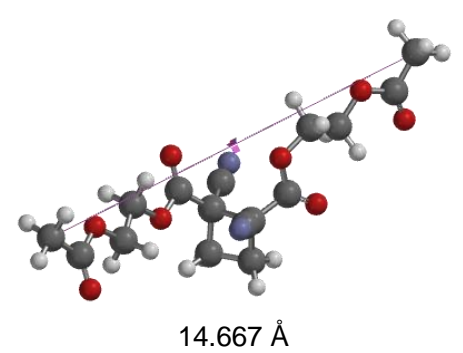

(ii) Immediately Prior to First Bond Cleavage

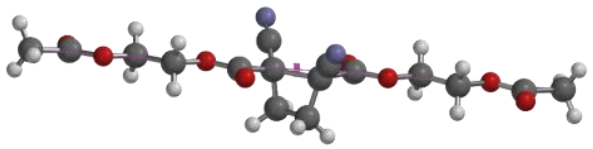

$20.417 \AA$

(iii) Immediately After First Bond Cleavage

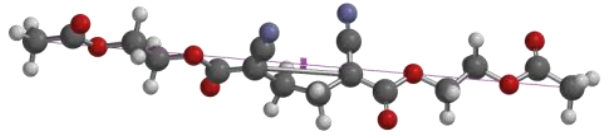

$20.467 \AA$

(iv) Immediately Prior to Second Bond Cleavage

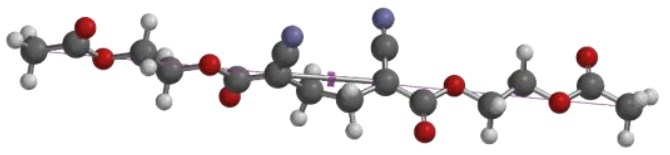

20.817

(iv) Immediately After Second Bond Cleavage

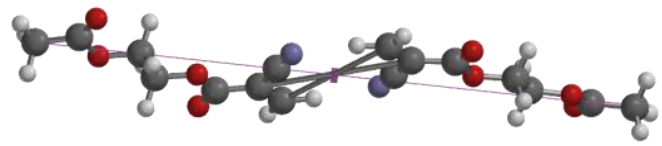

$20.867 \AA$ 


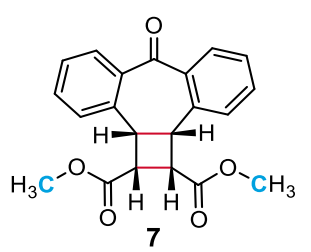

$\stackrel{\text { CoGEF }}{\longrightarrow}$$$
\text { . }
$$

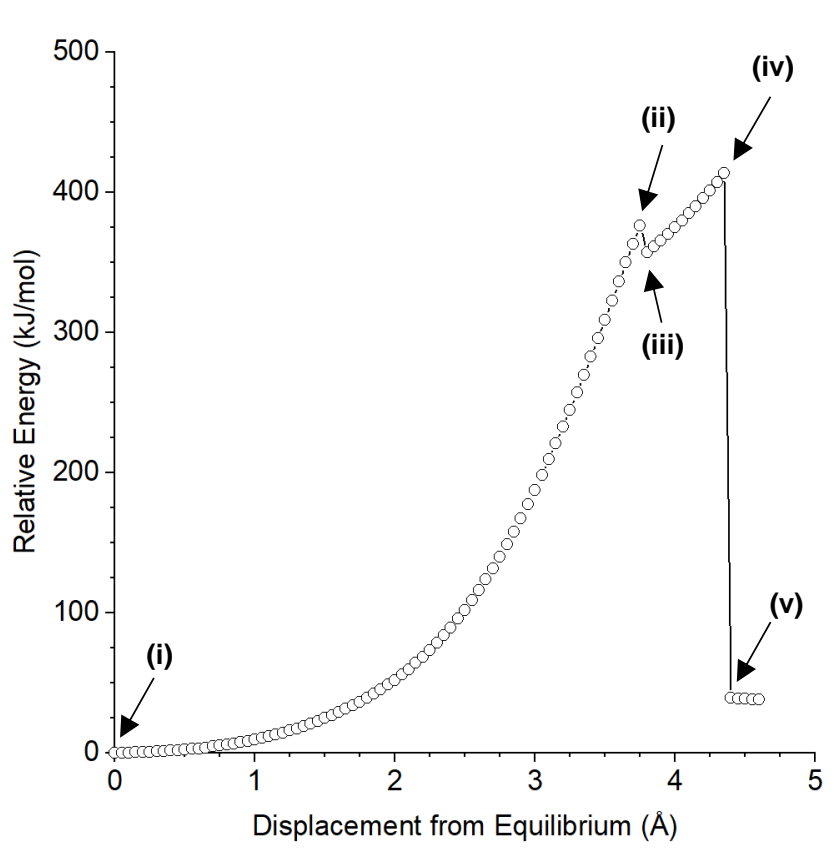

Summary of CoGEF Results

$F_{\text {max }} 4.5 \mathrm{nN}$

$E_{\max } \quad 413 \mathrm{~kJ} / \mathrm{mol}$

Force-Bond Angle $4.3^{\circ}$<smiles>COC(=O)/C=C\c1ccccc1C(=O)c1ccccc1/C=C/C(=O)OC</smiles>

(i) Equilibrium Geometry

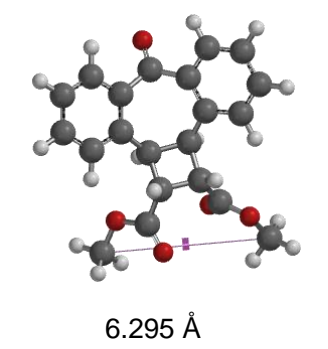

(ii) Immediately Prior to First Bond Cleavage

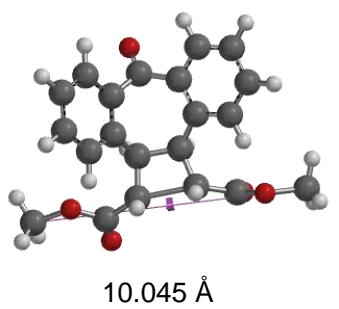

(iii) Immediately After First Bond Cleavage

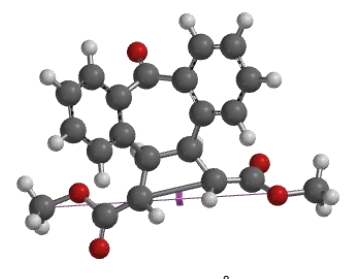

$10.095 \AA$

(iv) Immediately Before Second Bond Cleavage

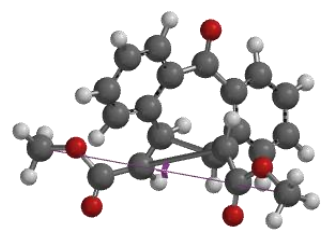

$10.645 \AA$

(v) Immediately After Second Bond Cleavage

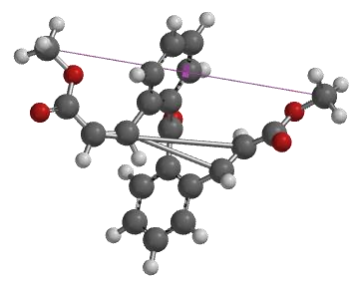

$10.695 \AA$ 


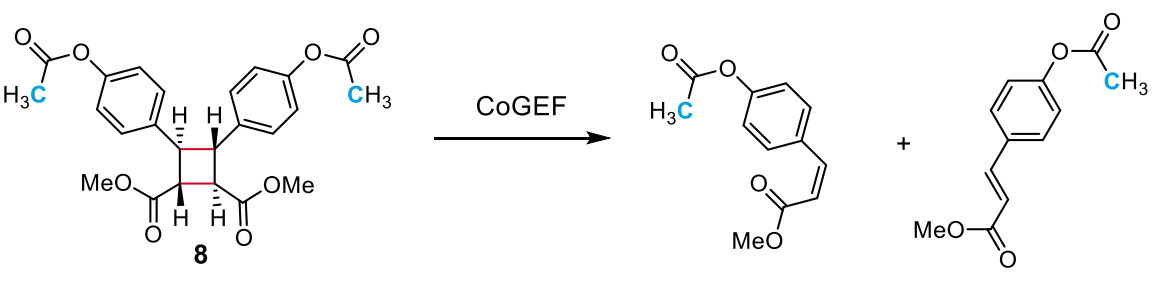

(i) Equilibrium Geometry
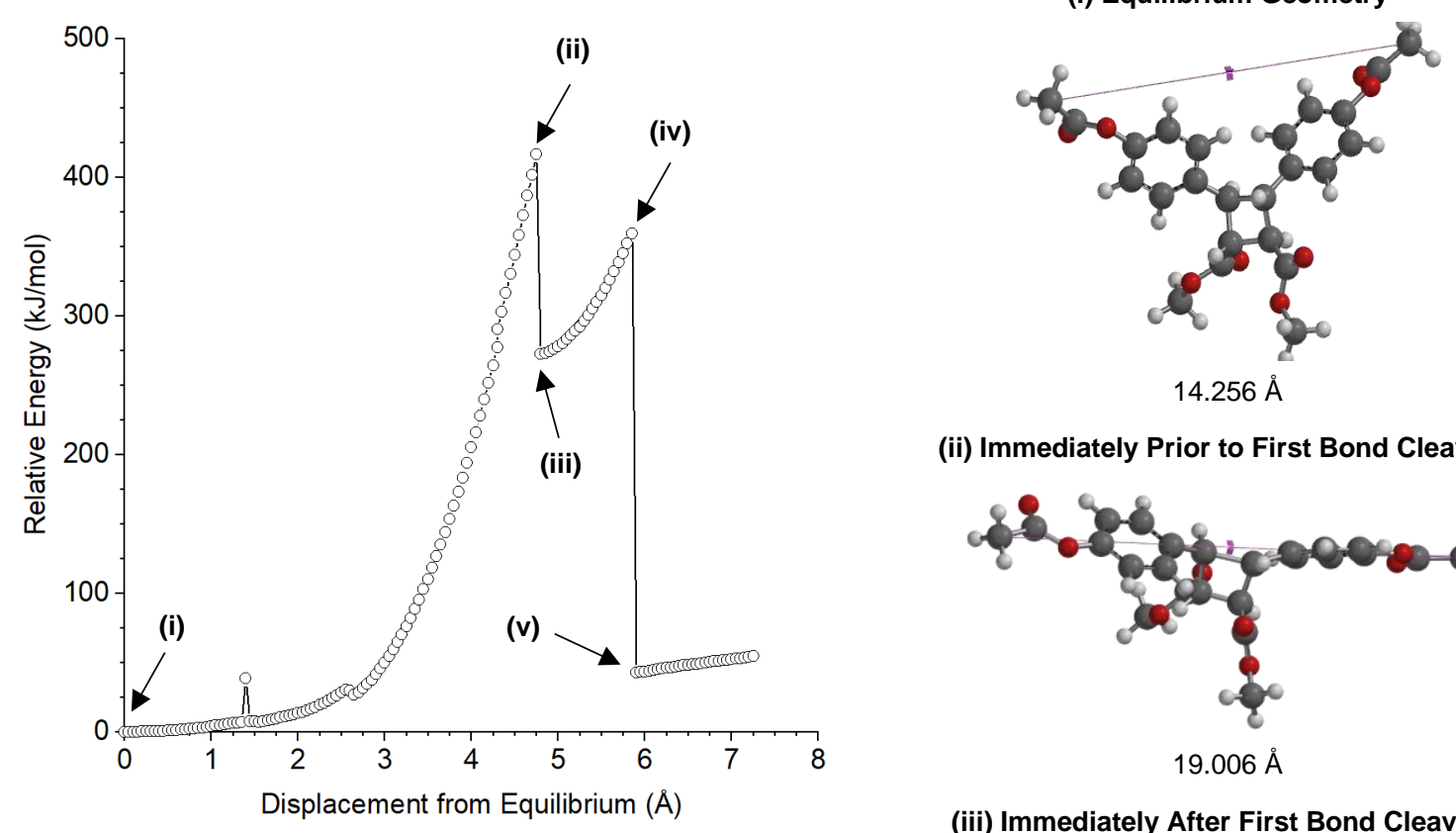

(ii) Immediately Prior to First Bond Cleavage

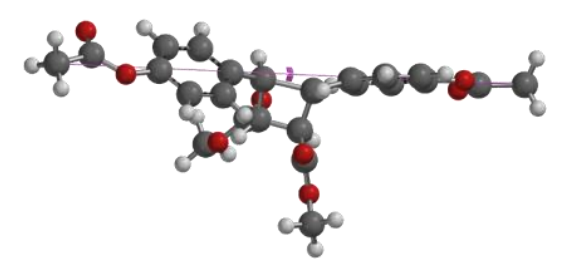

$19.006 \AA$

(iii) Immediately After First Bond Cleavage

Summary of CoGEF Results

$$
\begin{array}{ll}
\boldsymbol{F}_{\text {max }} & 5.0 \mathrm{nN} \\
\boldsymbol{E}_{\text {max }} & 417 \mathrm{~kJ} / \mathrm{mol}
\end{array}
$$

Force-Bond Angle $30^{\circ}$

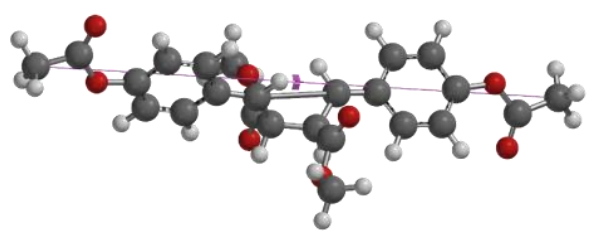

$19.056 \AA$

(iv) Immediately Prior to Second Bond Cleavage

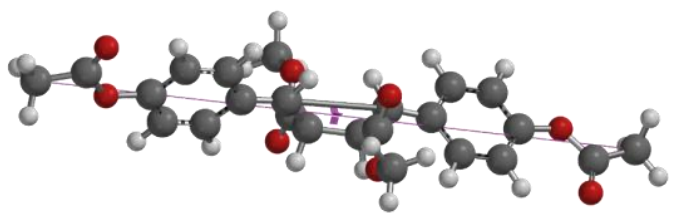

20.106

(v) Immediately After Second Bond Cleavage

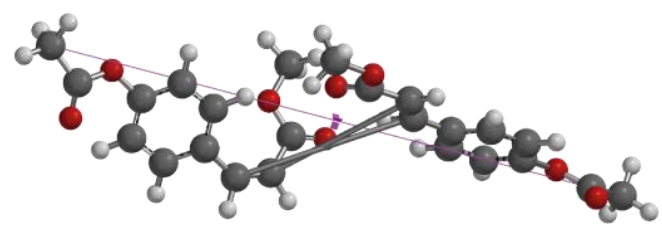

$20.156 \AA$ 

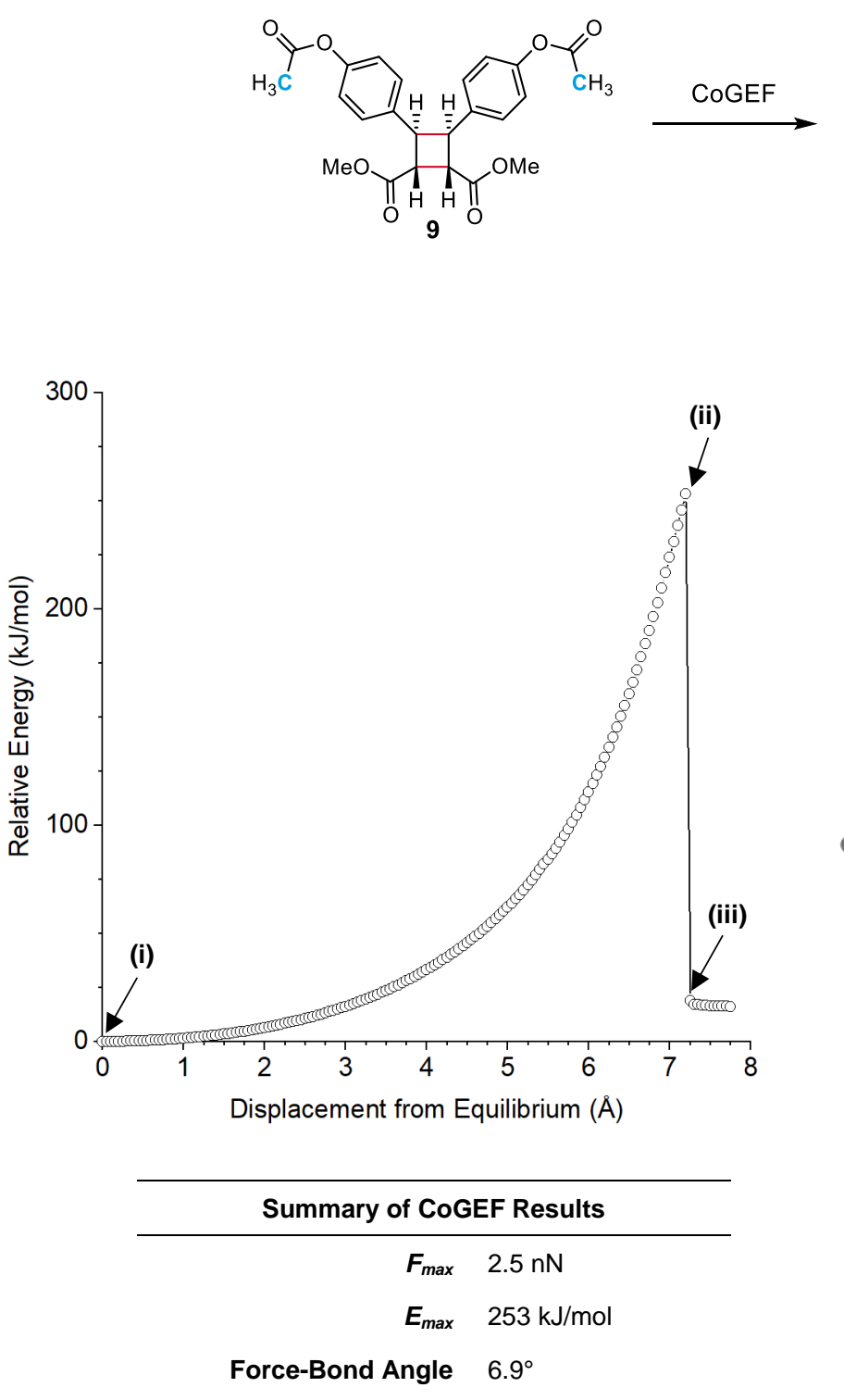<smiles>COC(=O)/C=C/c1ccc(OC(C)=O)cc1</smiles>

(i) Equilibrium Geometry

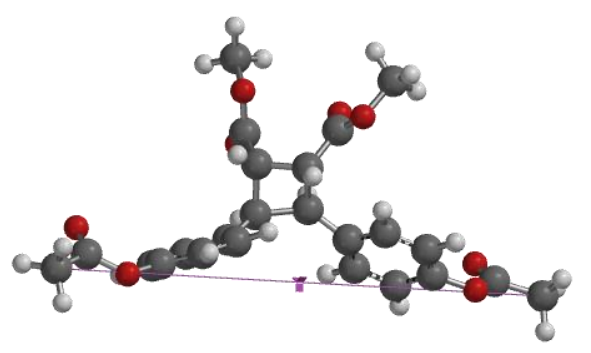

$10.962 \AA$

(ii) Immediately Prior to First Bond Cleavage

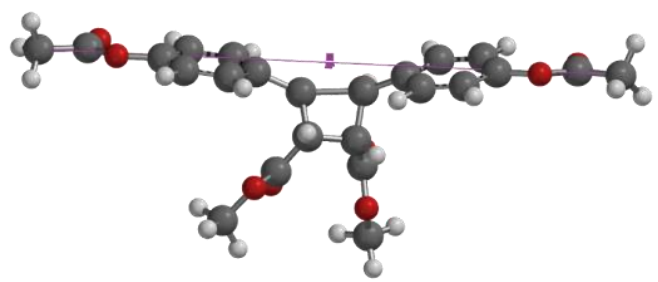

$18.162 \AA$

(iii) Immediately After First Bond Cleavage

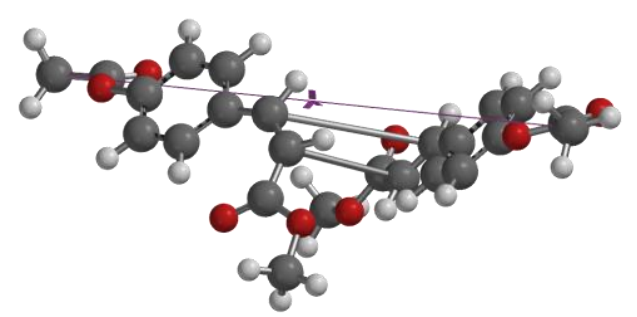

$18.212 \AA$ 

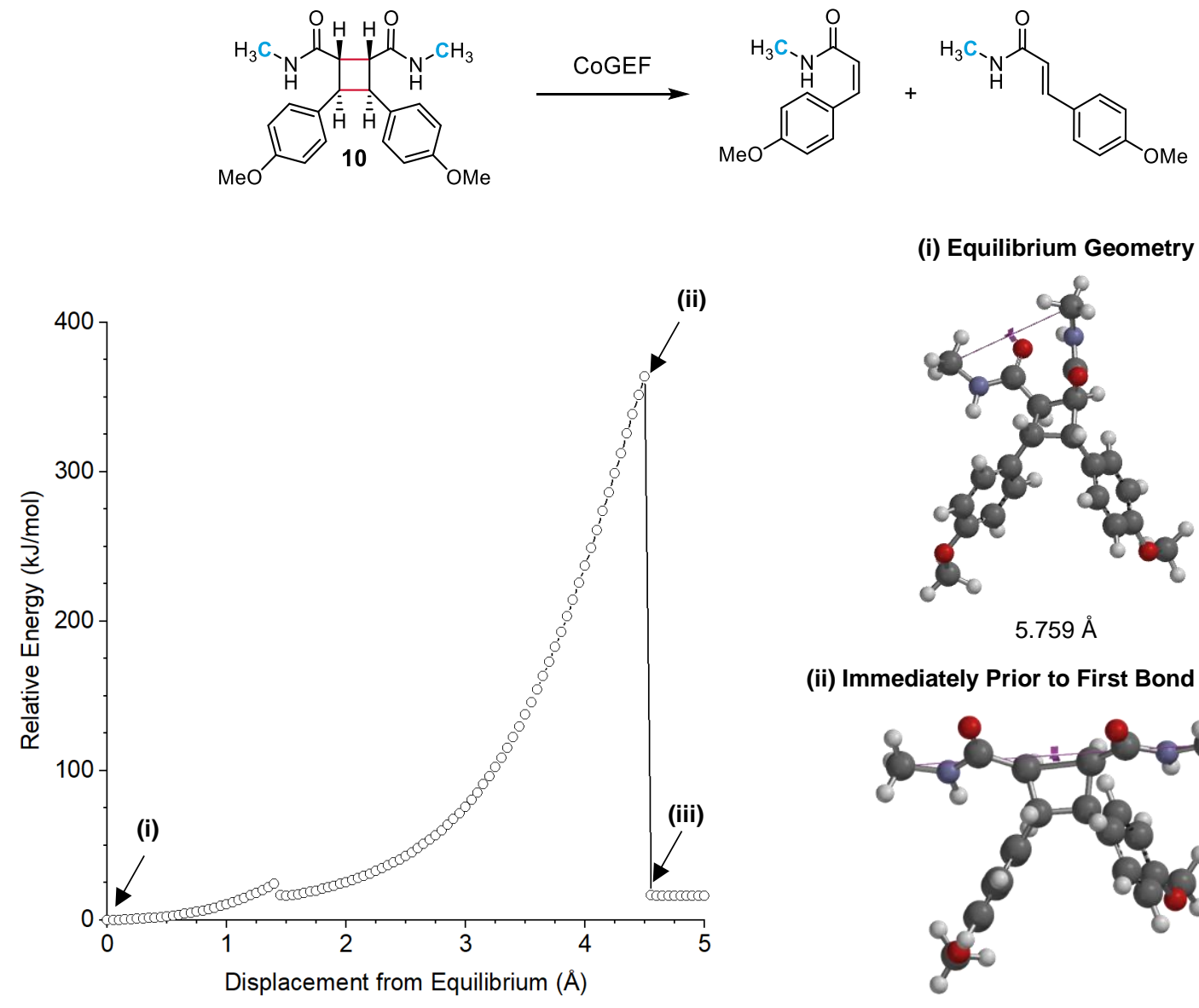

(i) Equilibrium Geometry

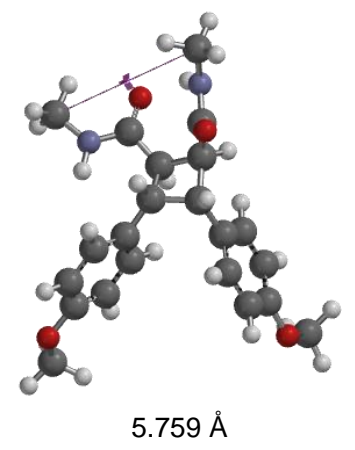

(ii) Immediately Prior to First Bond Cleavage

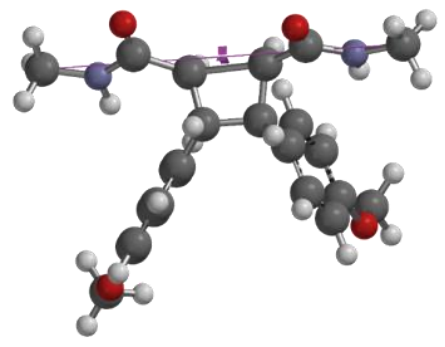

$10.259 \AA$

(iii) Immediately After First Bond Cleavage

Summary of CoGEF Results

$$
\begin{array}{ll}
\boldsymbol{F}_{\max } & 4.4 \mathrm{nN} \\
\boldsymbol{E}_{\max } & 364 \mathrm{~kJ} / \mathrm{mol}
\end{array}
$$

Force-Bond Angle $2.7^{\circ}$

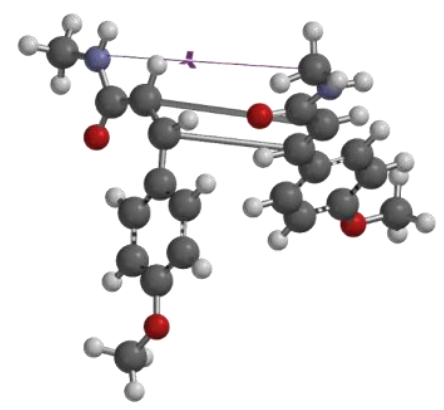

$10.309 \AA$ 


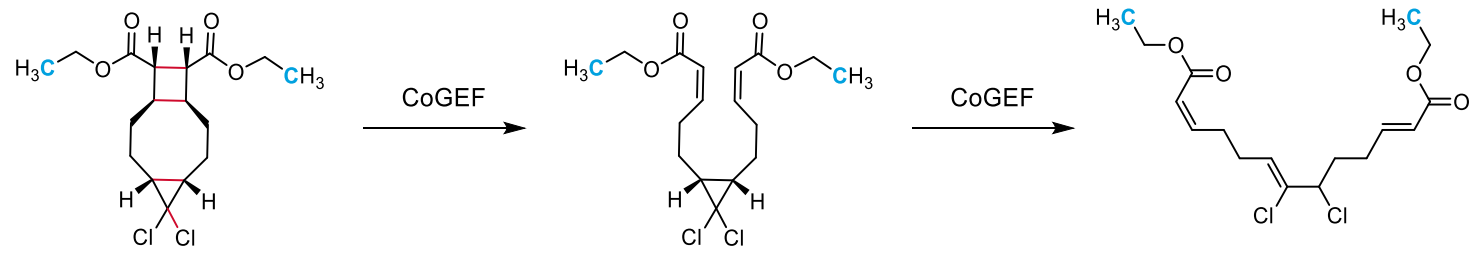

11

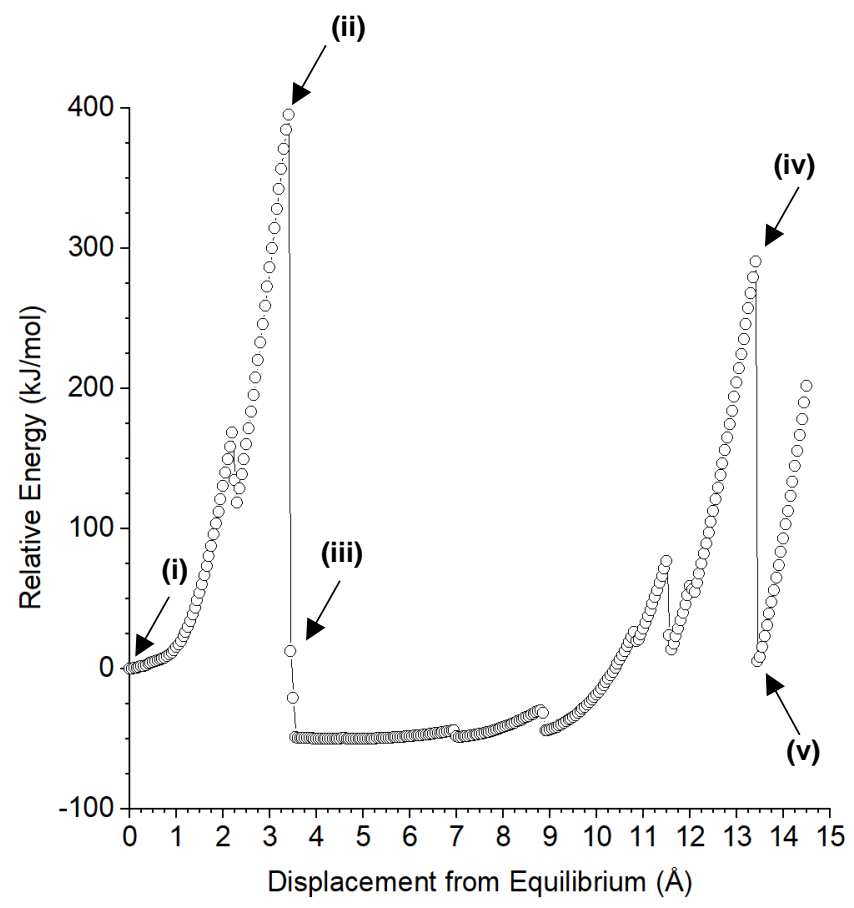

(i) Equilibrium Geometry

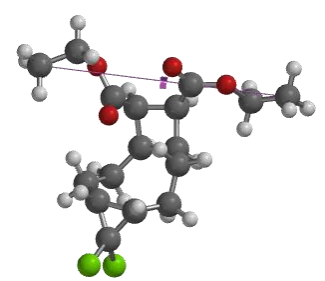

$9.485 \AA$

(ii) Immediately Prior to First Bond Cleavage

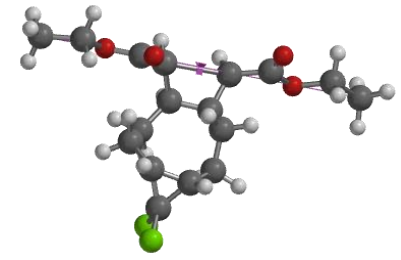

$12.885 \AA$

(iii) Immediately After First Bond Cleavage

\begin{tabular}{ccc}
\hline \multicolumn{3}{c}{ Summary of CoGEF Results } \\
\hline & cyclobutane & $g \mathrm{DCC}$ \\
\hline $\boldsymbol{F}_{\max }$ & $4.7 \mathrm{nN}$ & $3.8 \mathrm{nN}$ \\
$\boldsymbol{E}_{\max }$ & $395 \mathrm{~kJ} / \mathrm{mol}$ & $291 \mathrm{~kJ} / \mathrm{mol}$ \\
Force-Bond Angle & $2.1^{\circ}$ & $0.4^{\circ}$
\end{tabular}

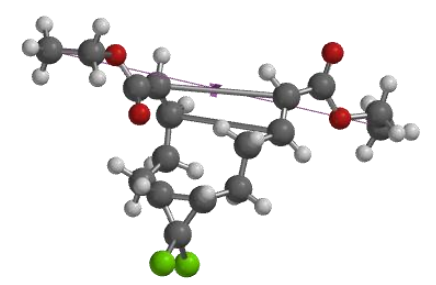

$12.935 \AA$

(iv) Immediately Before Second Bond Cleavage

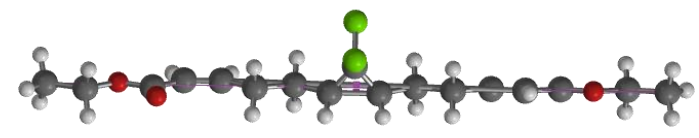

$22.885 \AA$

(v) Immediately After Second Bond Cleavage

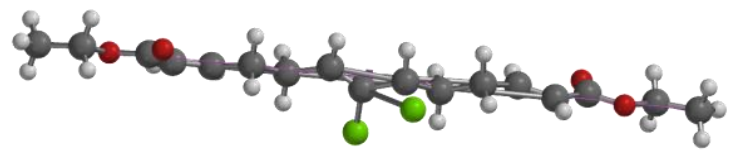

$22.935 \AA$ 

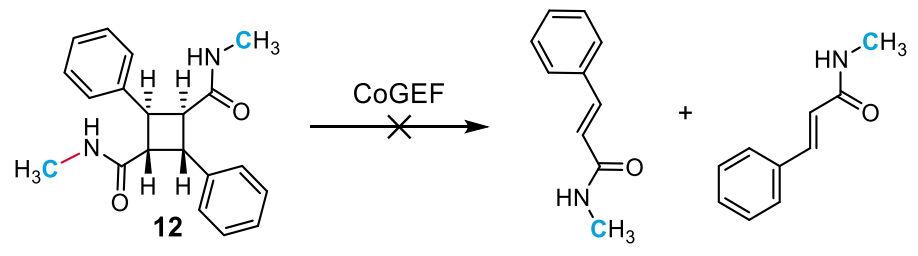

(i) Equilibrium Geometry
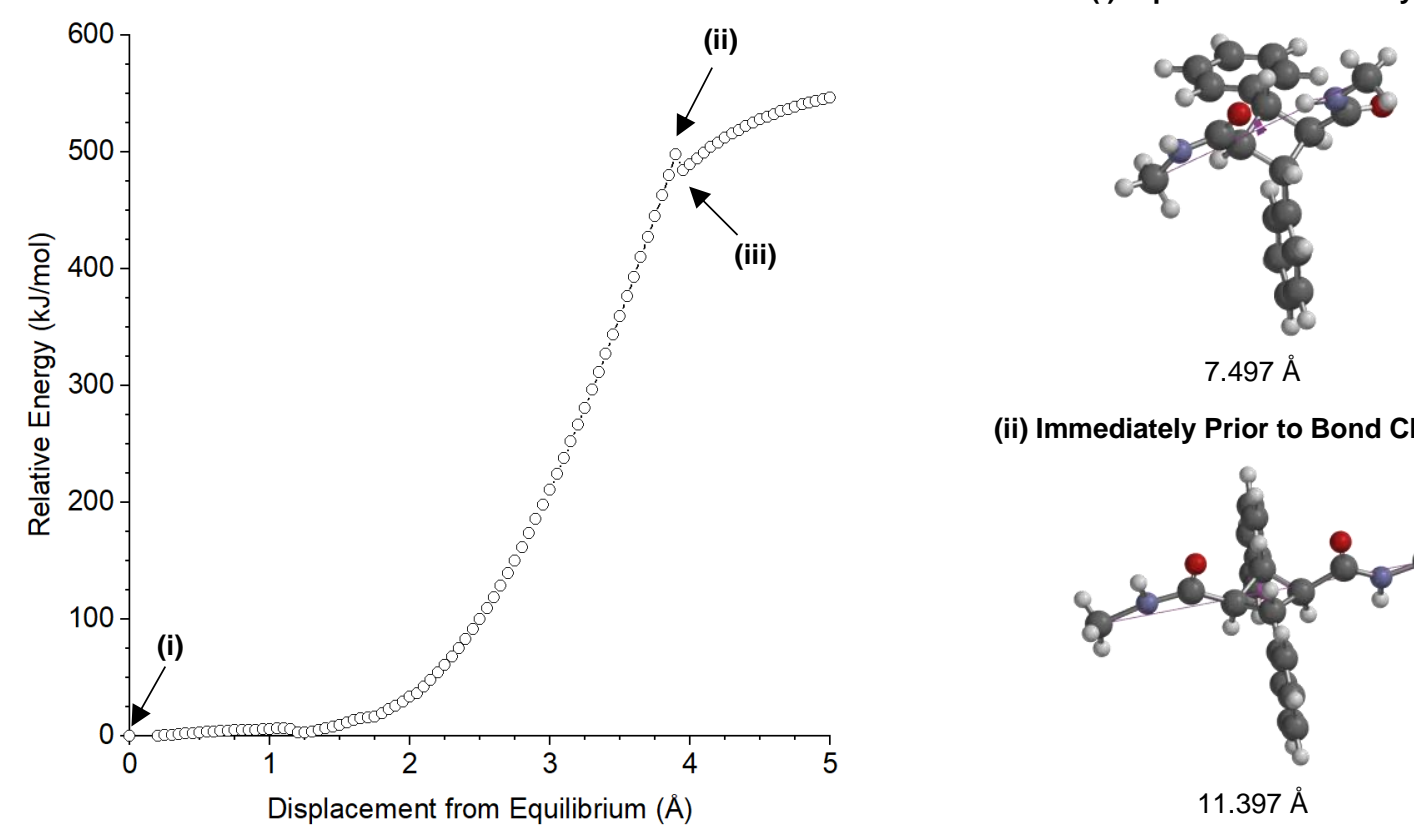

(ii) Immediately Prior to Bond Cleavage

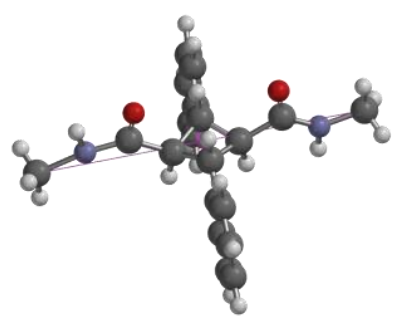

$11.397 \AA$

(iii) Immediately After Bond Cleavage

Summary of CoGEF Results

$\begin{array}{ll}\boldsymbol{F}_{\text {max }} & 5.9 \mathrm{nN} \\ \boldsymbol{E}_{\max } & 498 \mathrm{~kJ} / \mathrm{mol}\end{array}$

Force-Bond Angle $44^{\circ}$

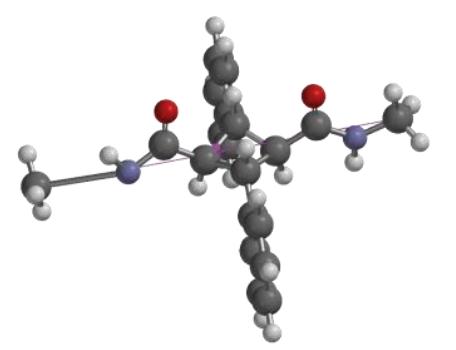

$11.5447 \AA$ 


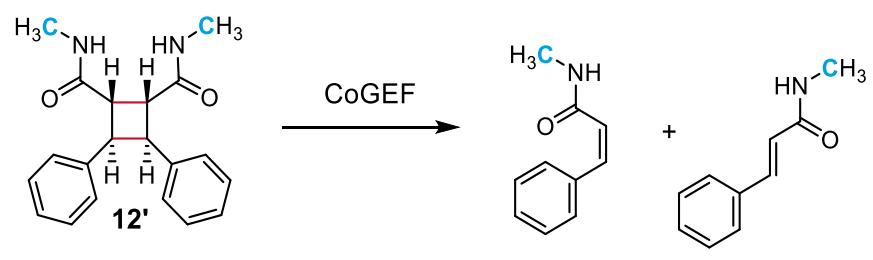

(ii)

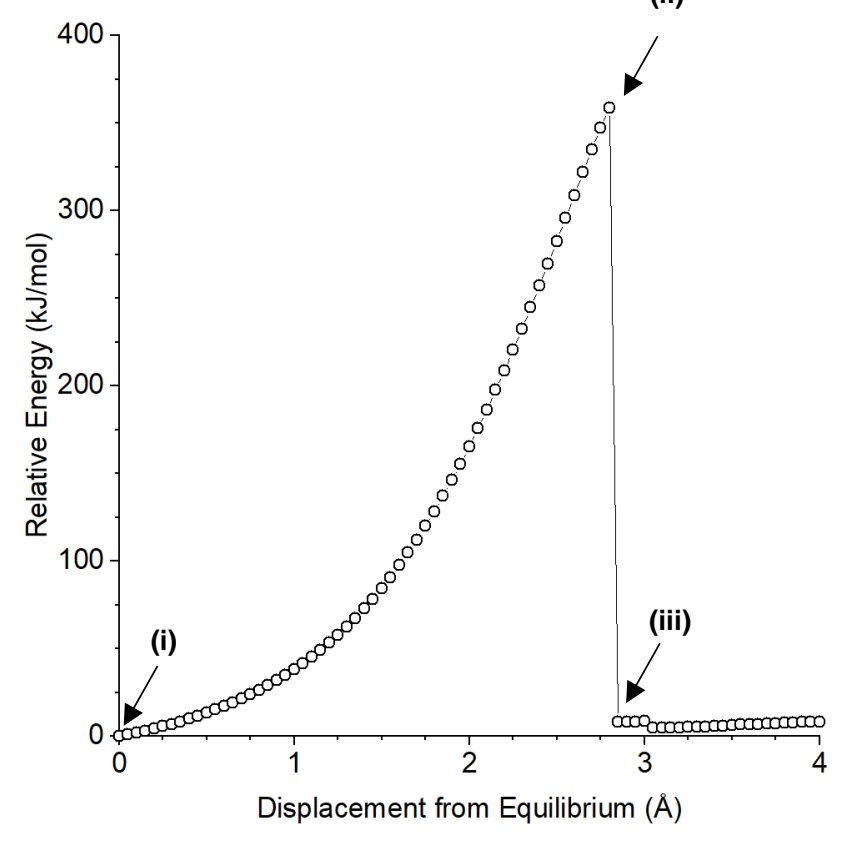

(i) Equilibrium Geometry

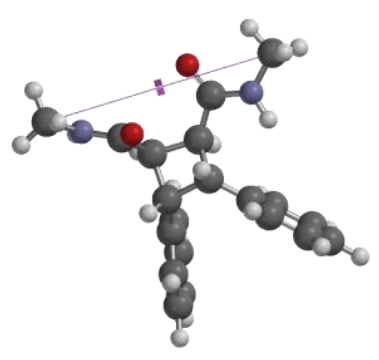

$7.519 \AA$

(ii) Immediately Prior to Bond Cleavage

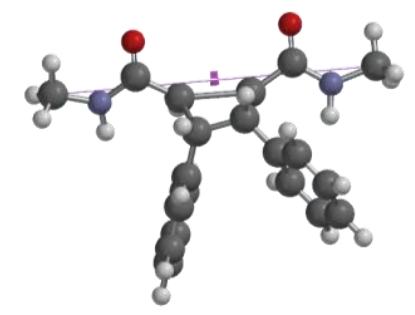

$10.319 \AA$

(iii) Immediately After Bond Cleavage

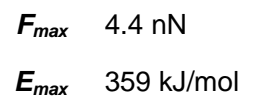

Force-Bond Angle $2.2^{\circ}$

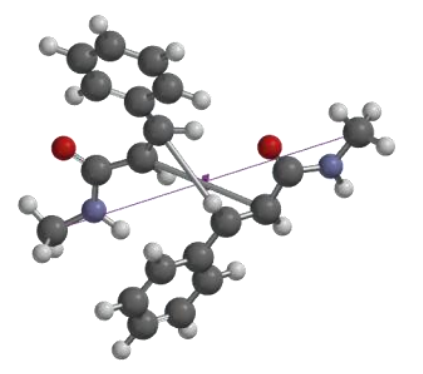

$10.369 \AA$ 

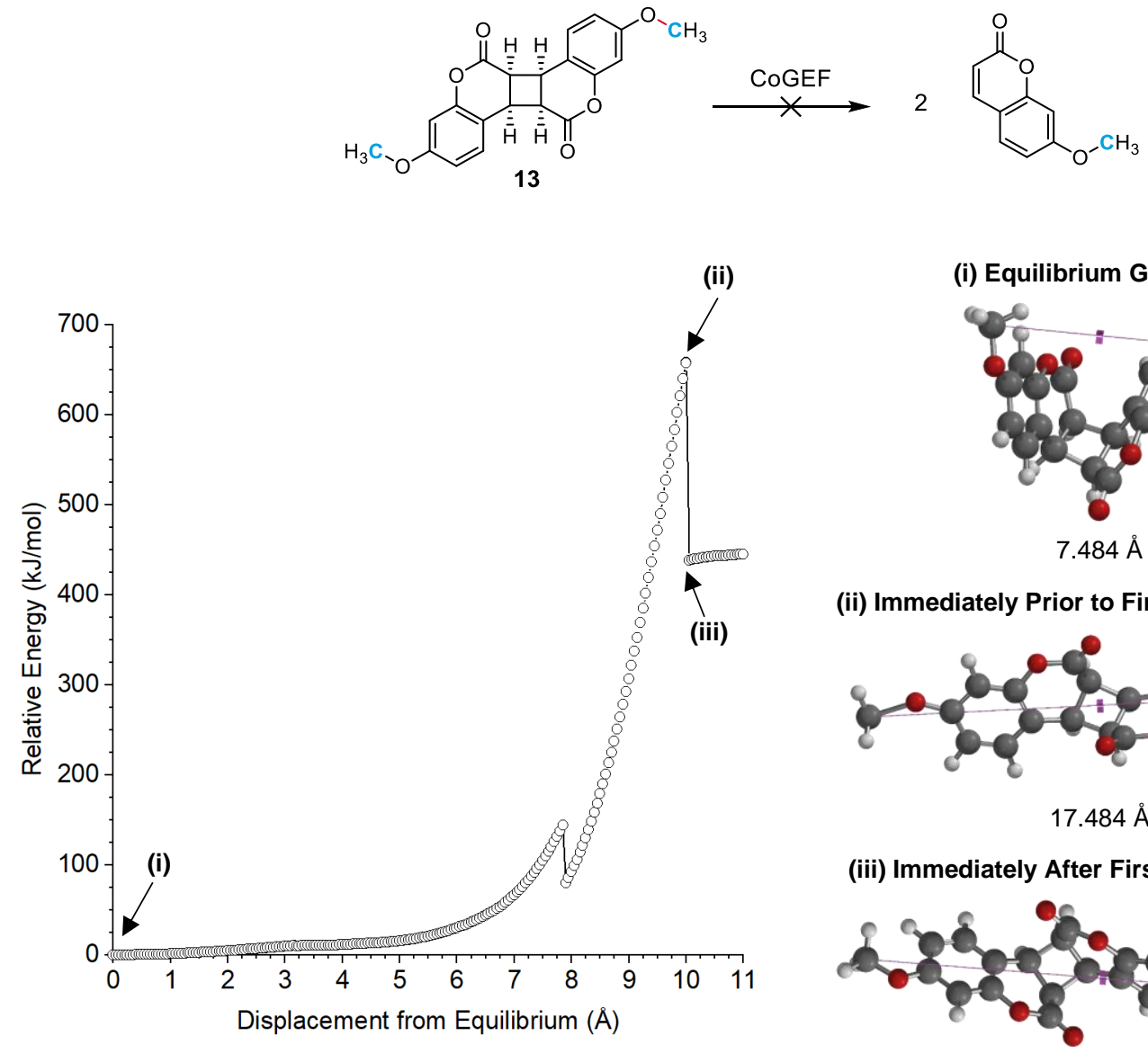

(i) Equilibrium Geometry

(ii)

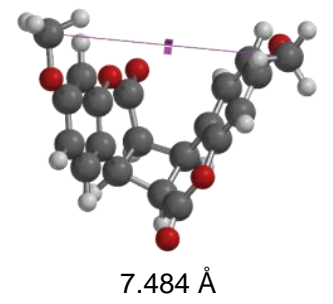

(ii) Immediately Prior to First Bond Cleavage

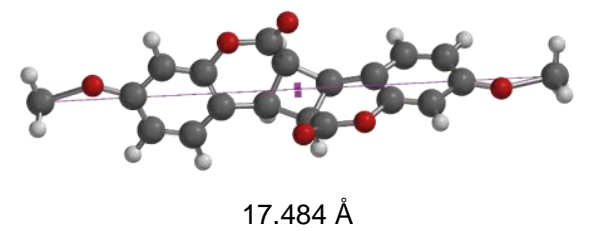

(iii) Immediately After First Bond Cleavage

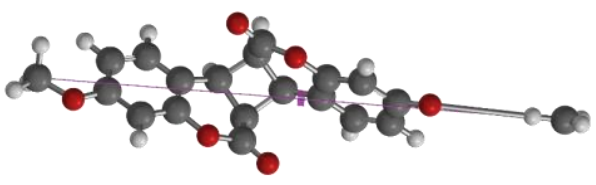

$17.534 \AA$

Summary of CoGEF Results

$\boldsymbol{F}_{\max } \quad 6.3 \mathrm{nN}$

$E_{\max } \quad 658 \mathrm{~kJ} / \mathrm{mol}$

Force-Bond Angle $28^{\circ}$ 

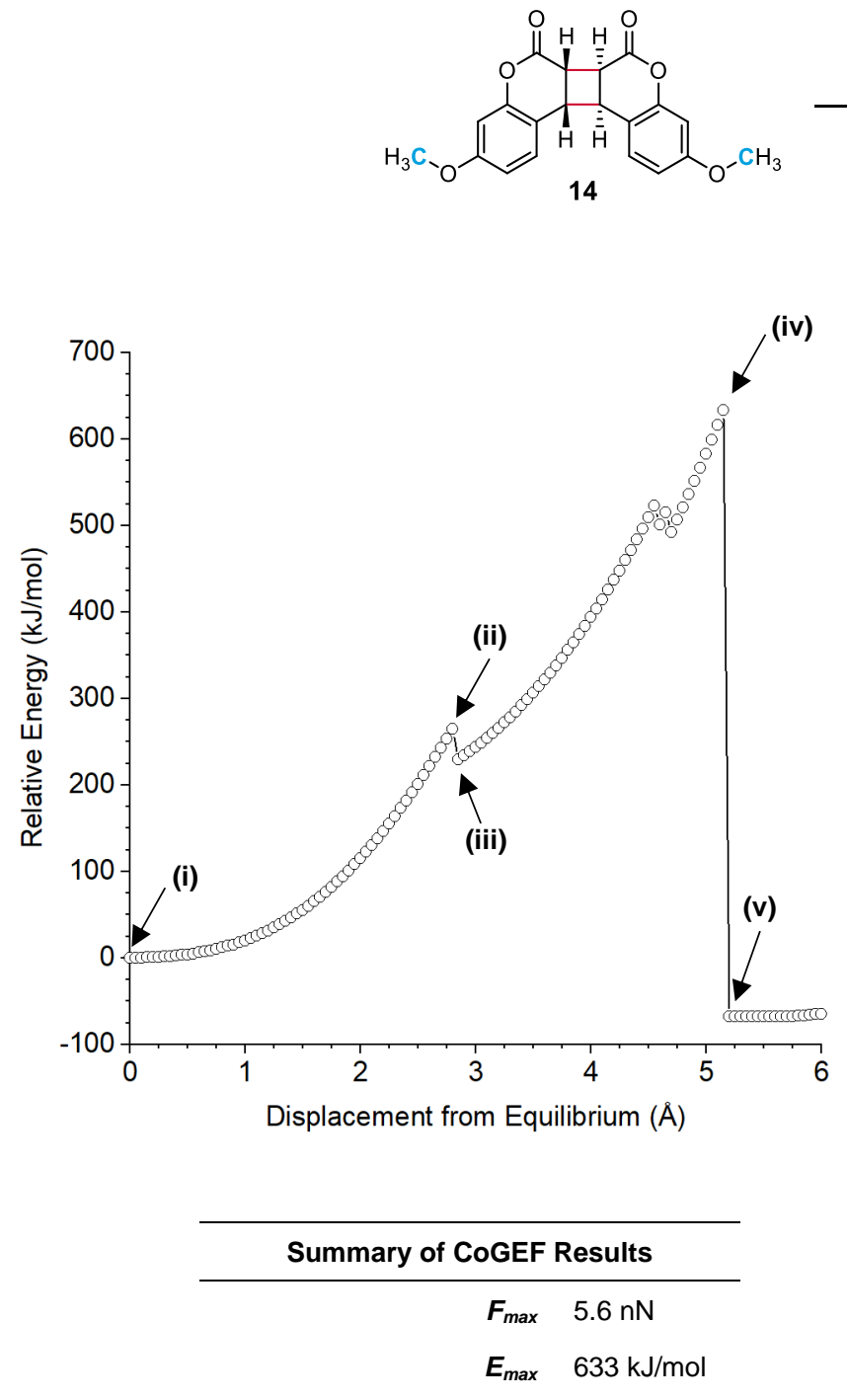

Force-Bond Angle $26^{\circ}$

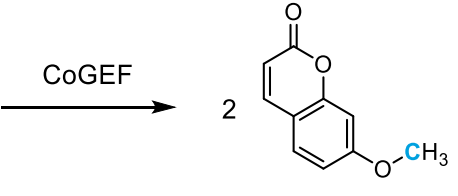

(i) Equilibrium Geometry

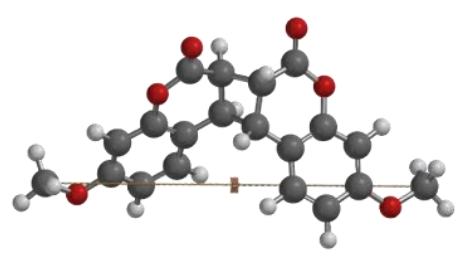

$13.066 \AA$

(ii) Immediately Prior to First Bond Cleavage

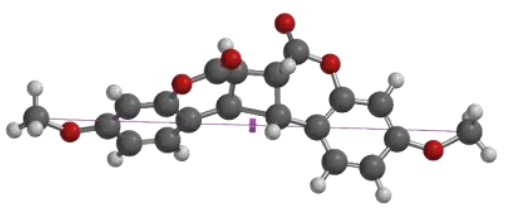

$15.866 \AA$

(iii) Immediately After First Bond Cleavage

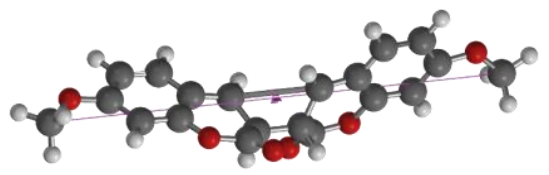

$15.916 \AA$

(iv) Immediately Before Second Bond Cleavage

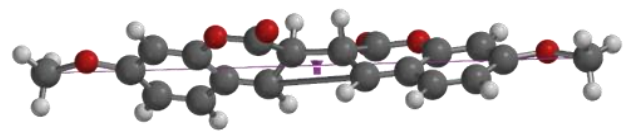

$18.216 \AA$

(v) Immediately After Second Bond Cleavage

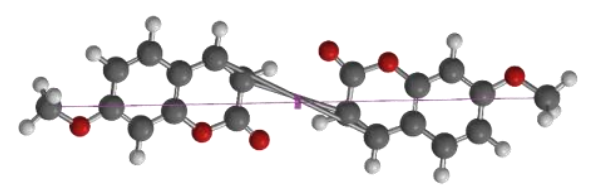

$18.266 \AA$ 
<smiles>CC(=O)OCCOc1ccc2c(c1)OC(=O)[C@@H]1[C@@H]3C(=O)Oc4cc(OCCOC(C)=O)ccc4[C@@]3(C)[C@@H]21</smiles>

(iv)

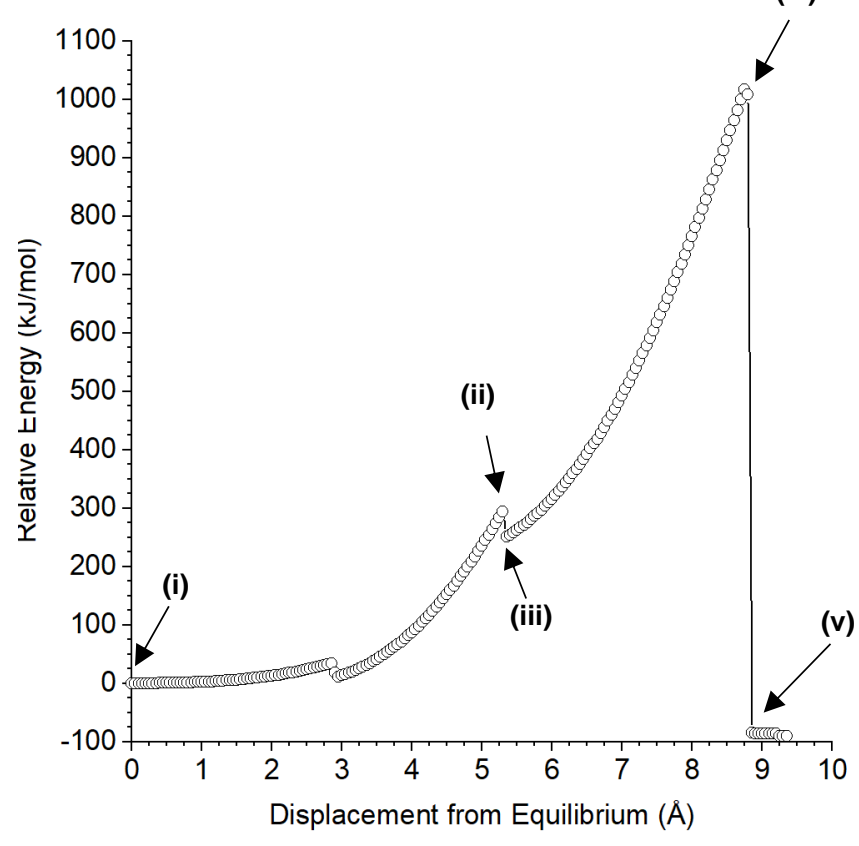

(i) Equilibrium Geometry

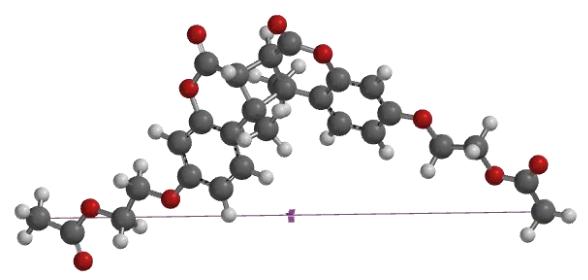

$20.607 \AA$

(ii) Immediately Prior to First Bond Cleavage

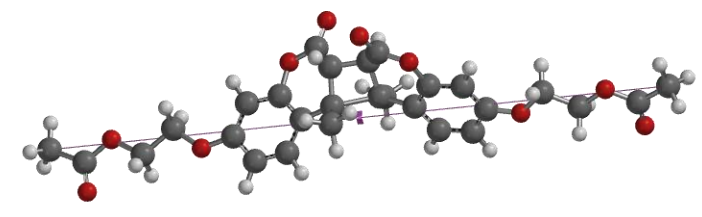

$25.907 \AA$

(iii) Immediately After First Bond Cleavage

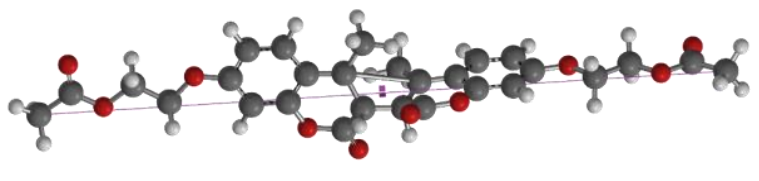

$25.957 \AA$

(iv) Immediately Before Second Bond Cleavage

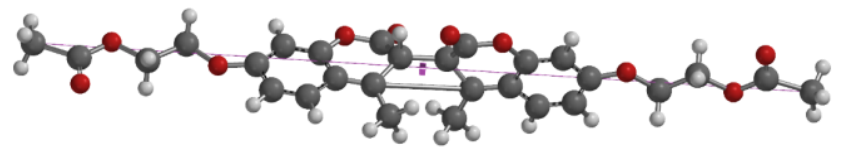

$29.407 \AA$

(v) Immediately After Second Bond Cleavage

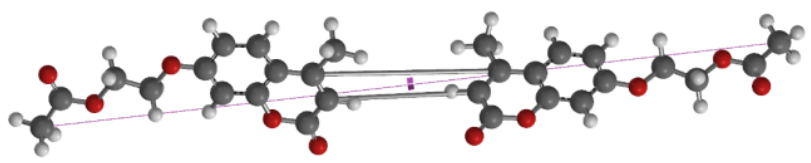

$29.457 \AA$ 


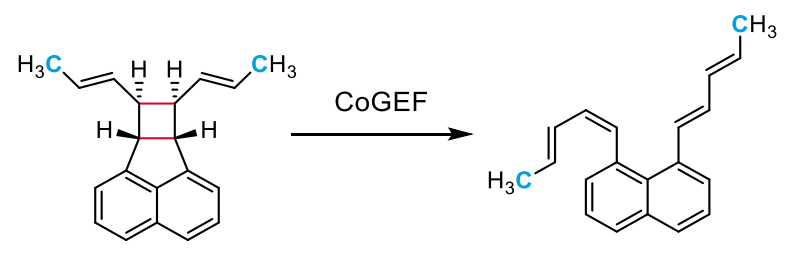

16

(i) Equilibrium Geometry

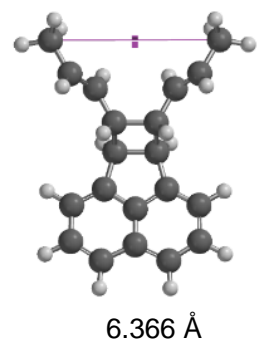

(v) Immediately Prior to Second Bond Cleavage

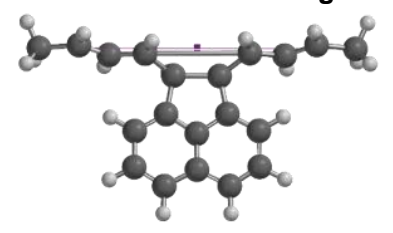

$12.216 \AA$ (ii) Immediately Prior to First Bond Cleavage

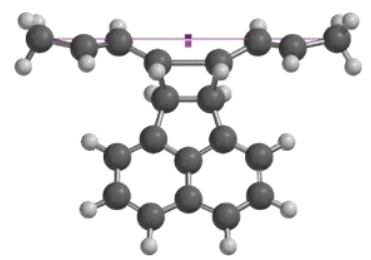

$10.066 \AA$ (iii) Immediately After First Bond

Cleavage

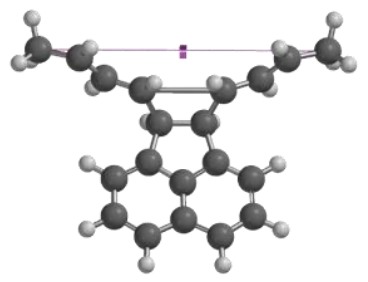

$10.116 \AA$

(vi) Immediately After Second Bond Cleavage

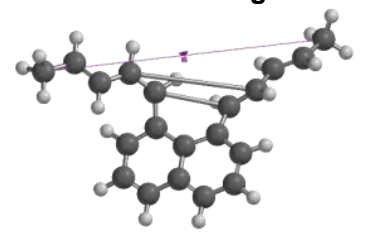

$12.266 \AA$

(iv)

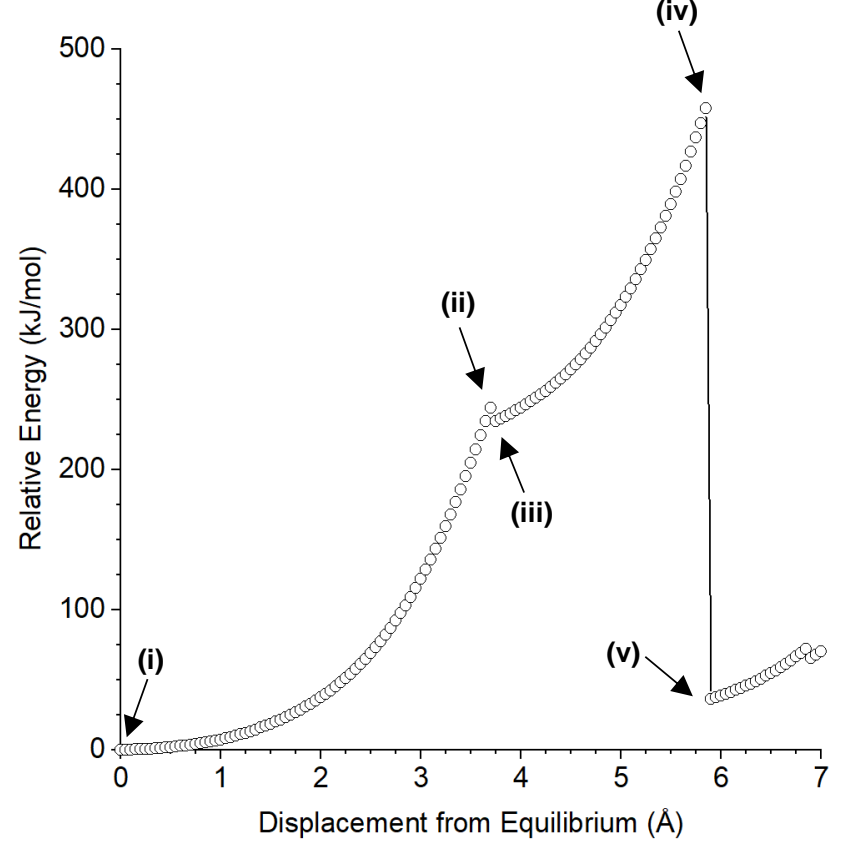

Summary of CoGEF Results

$\boldsymbol{F}_{\max } \quad 3.3 \mathrm{nN}$

$E_{\max } 244 \mathrm{~kJ} / \mathrm{mol}$

Force-Bond Angle $0.0^{\circ}$ 


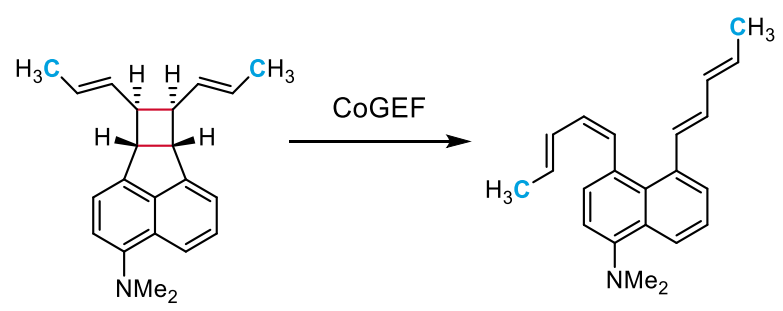

17

(i) Equilibrium Geometry

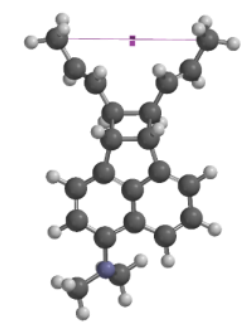

$6.351 \AA$

(v) Immediately Prior to Second Bond Cleavage

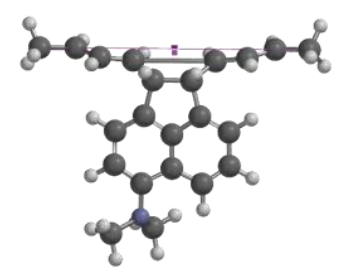

$11.351 \AA$ (ii) Immediately Prior to First Bond Cleavage

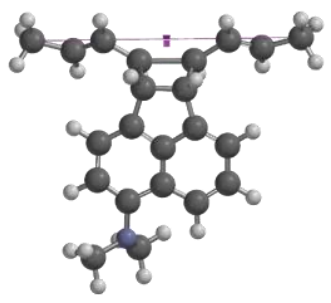

$10.051 \AA$ (iii) Immediately After First Bond

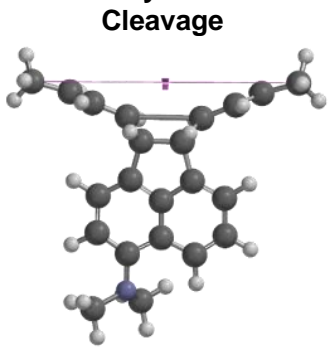

$10.101 \AA$

(vi) Immediately After Second Bond Cleavage

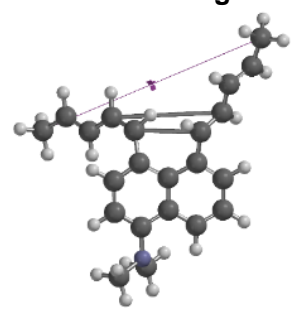

$11.401 \AA$

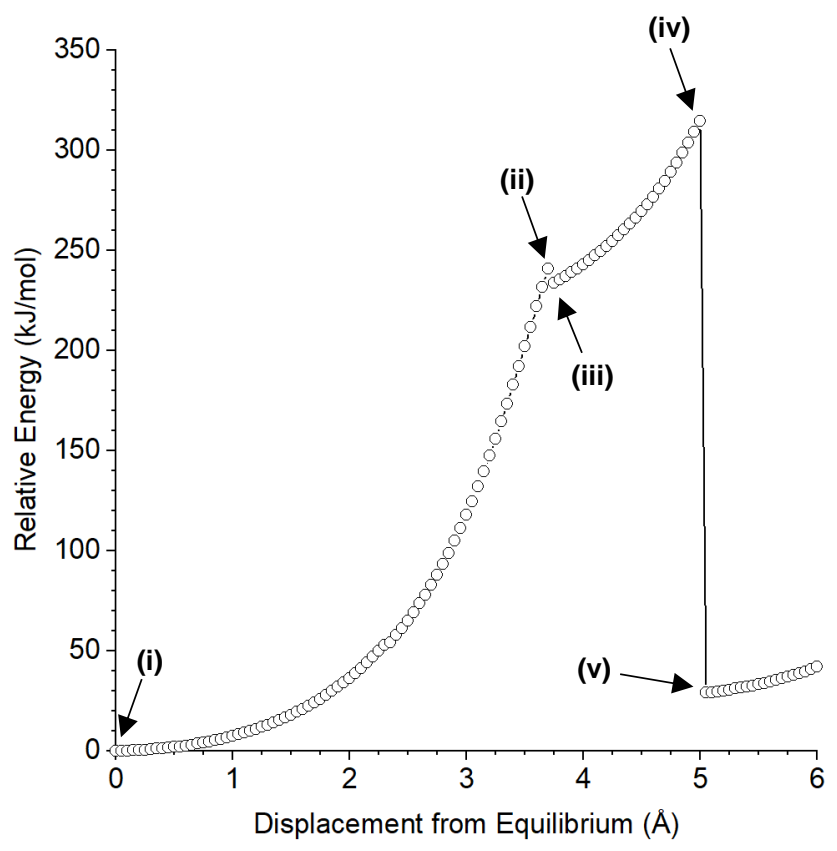

Summary of CoGEF Results

$\boldsymbol{F}_{\max } \quad 3.3 \mathrm{nN}$

$E_{\max } 241 \mathrm{~kJ} / \mathrm{mol}$

Force-Bond Angle $1.0^{\circ}$ 


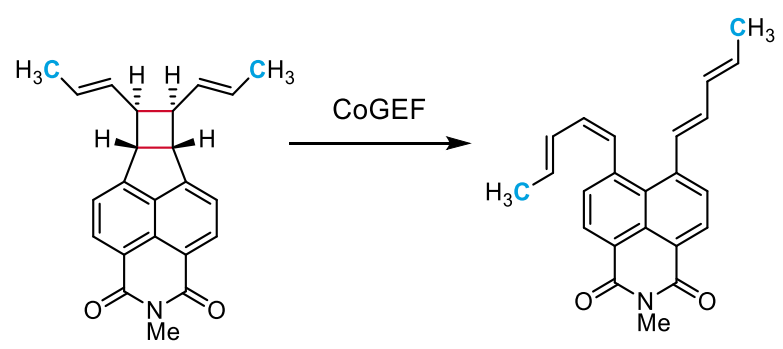

18

(i) Equilibrium Geometry

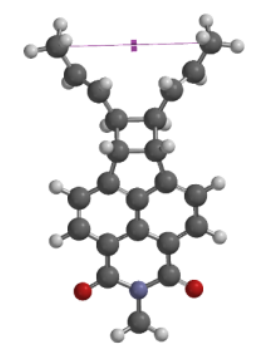

$6.319 \AA$

(v) Immediately Prior to Second Bond Cleavage

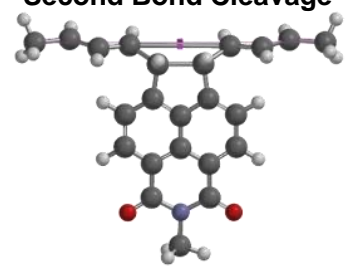

$12.569 \AA$ (ii) Immediately Prior to First Bond Cleavage

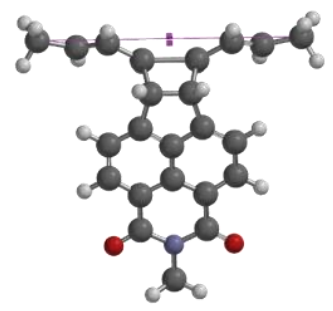

$10.069 \AA$

(vi) Immediately After Second

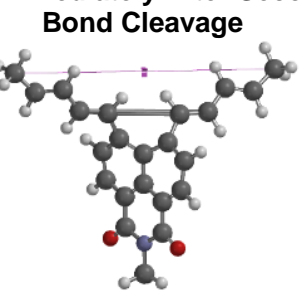

$12.619 \AA$ (iii) Immediately After First Bond Cleavage

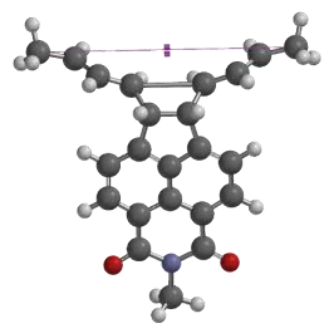

$10.119 \AA$

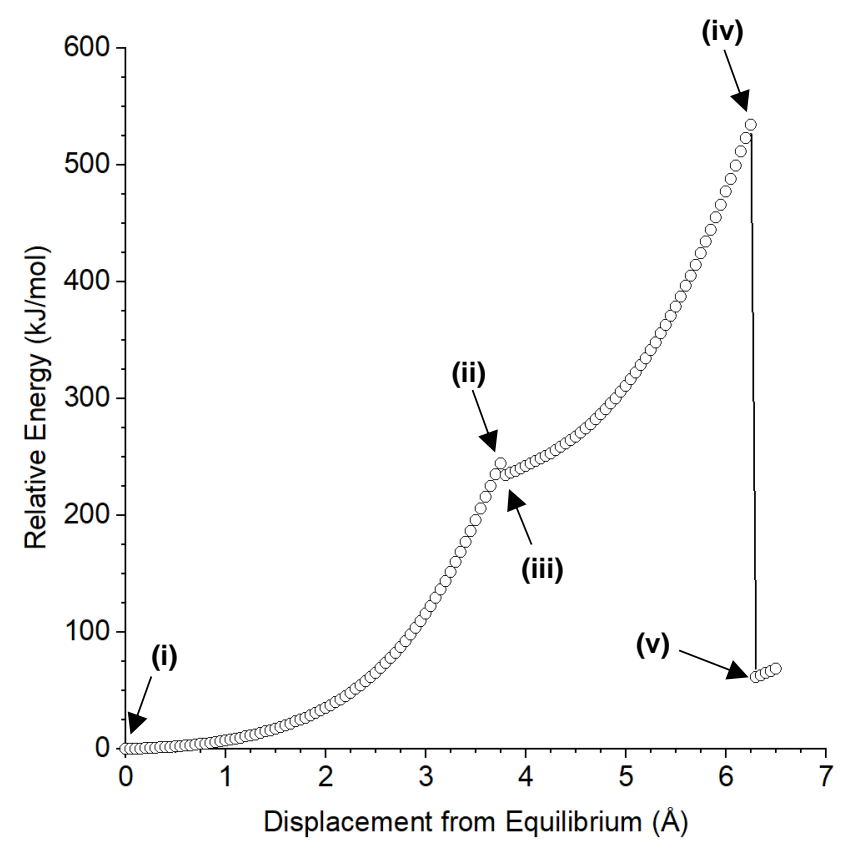




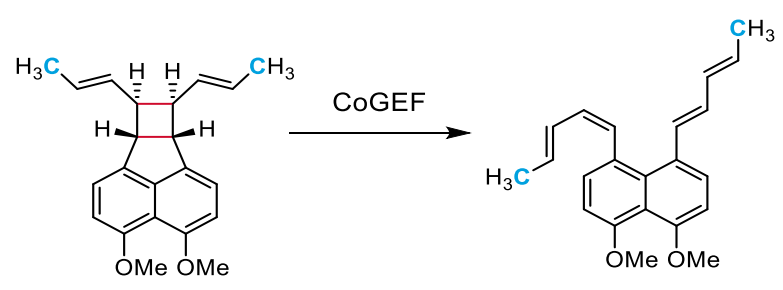

19

(i) Equilibrium Geometry

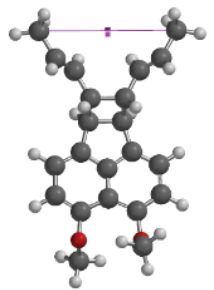

$6.325 \AA$

(v) Immediately Prior to Second Bond Cleavage

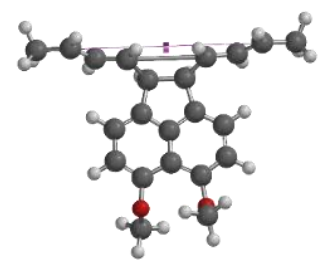

$11.325 \AA$ (ii) Immediately Prior to First Bond Cleavage

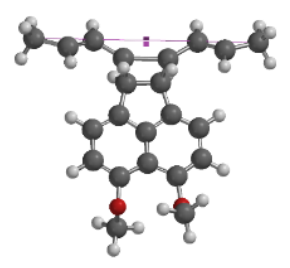

$10.025 \AA$ (iii) Immediately After First Bond Cleavage

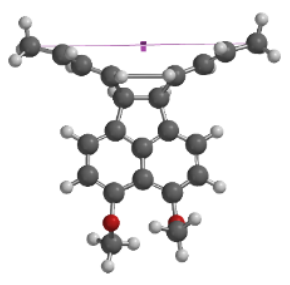

$10.075 \AA$

(vi) Immediately After Second Bond Cleavage

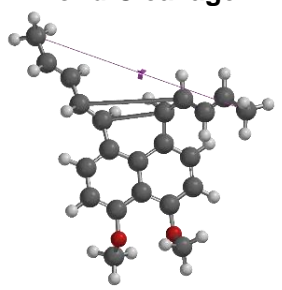

$11.375 \AA$

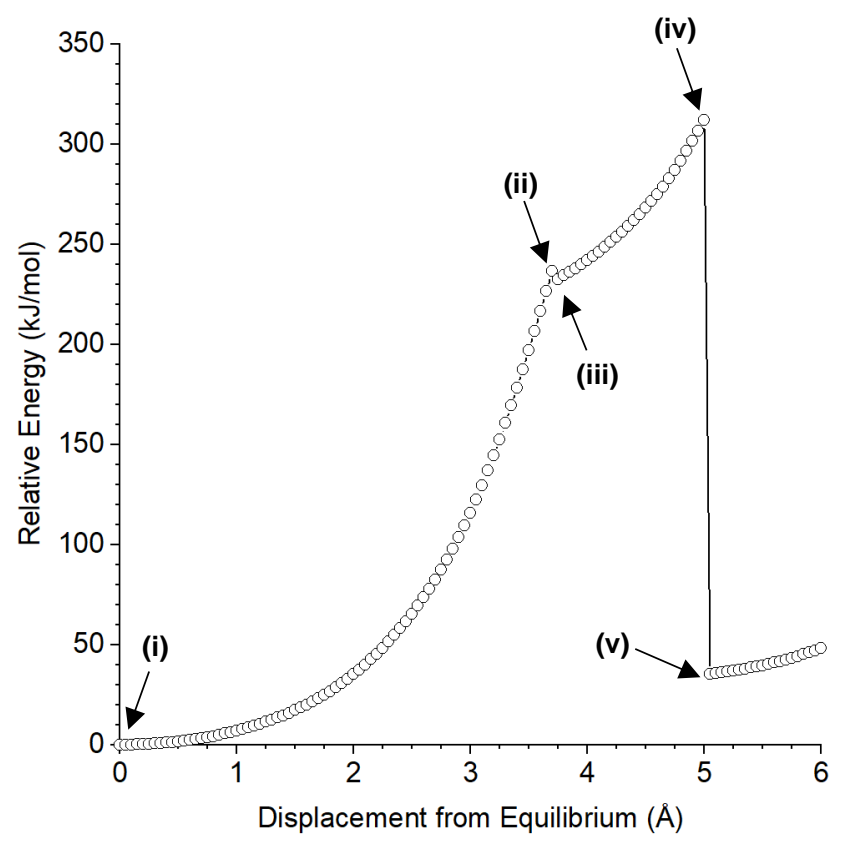

Summary of CoGEF Results

$\boldsymbol{F}_{\max } 3.3 \mathrm{nN}$

$E_{\max } 236 \mathrm{~kJ} / \mathrm{mol}$

Force-Bond Angle $1.2^{\circ}$ 

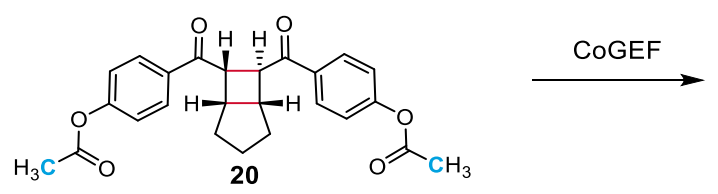<smiles>CC(=O)Oc1ccc(C(=O)/C=C/CCC/C=C/C(=O)c2ccc(OC(C)=O)cc2)cc1</smiles>

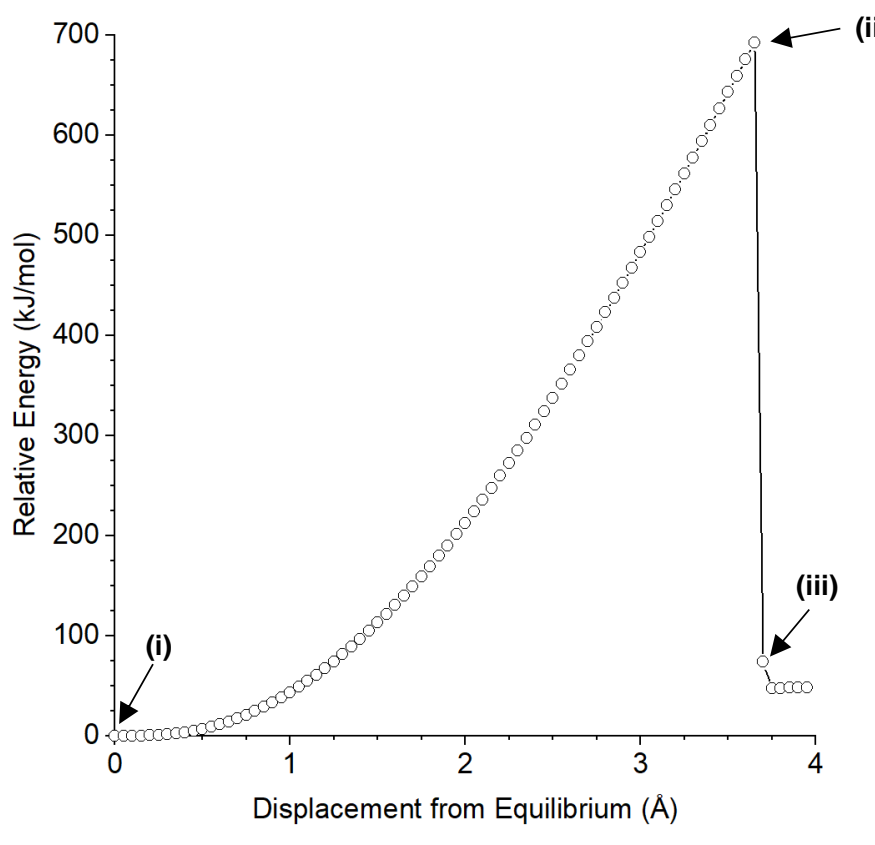

(i) Equilibrium Geometry

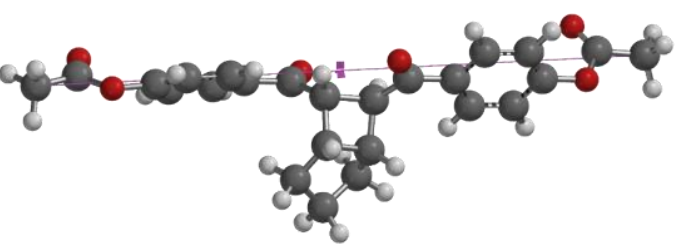

$18.900 \AA$

(i) Immediately Prior to Bond Cleavage

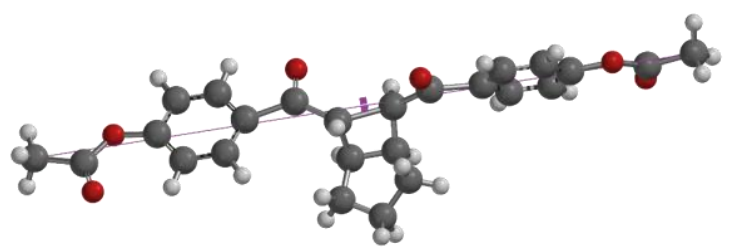

$22.550 \AA$

Summary of CoGEF Results

$$
\begin{array}{ll}
\boldsymbol{F}_{\text {max }} & 5.5 \mathrm{nN} \\
\boldsymbol{E}_{\text {max }} & 692 \mathrm{~kJ} / \mathrm{mol}
\end{array}
$$

Force-Bond Angle $16^{\circ}$

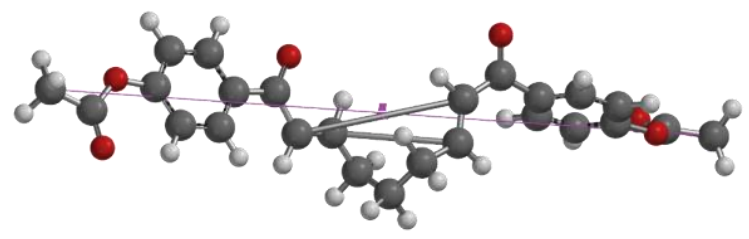

$22.600 \AA$ 


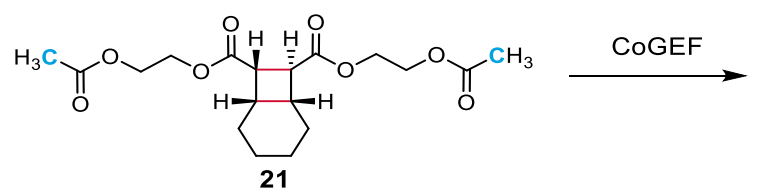<smiles>CC(=O)OCCOC(=O)/C=C/C=C/C(=O)OCCOC(C)=O</smiles>

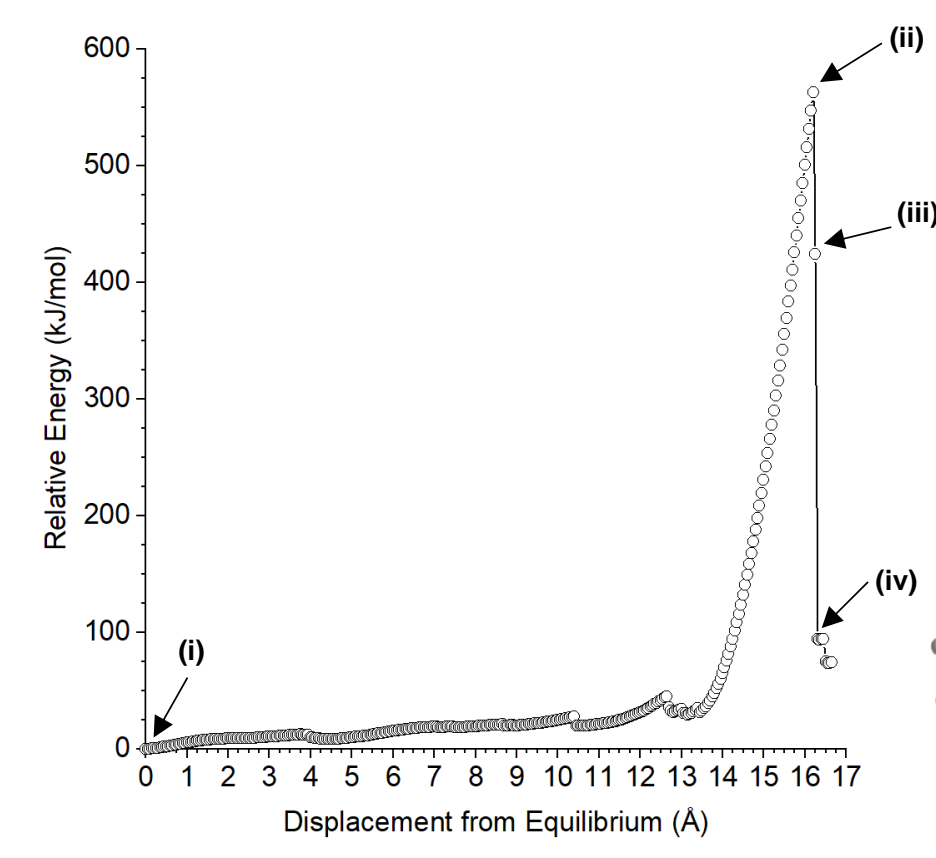

(i) Equilibrium Geometry

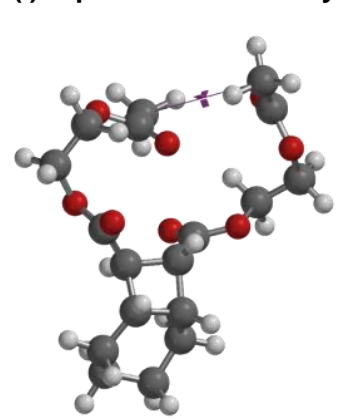

$4.462 \AA$

(ii) Immediately Prior to First Bond Cleavage

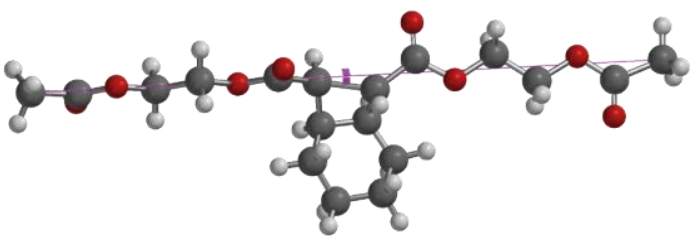

$20.662 \AA$

(iii) Immediately After First Bond Cleavage

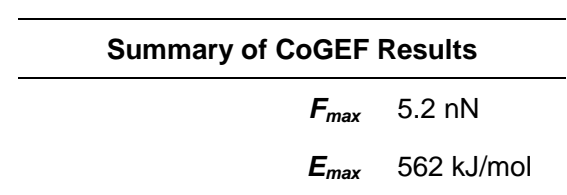

Force-Bond Angle $17^{\circ}$

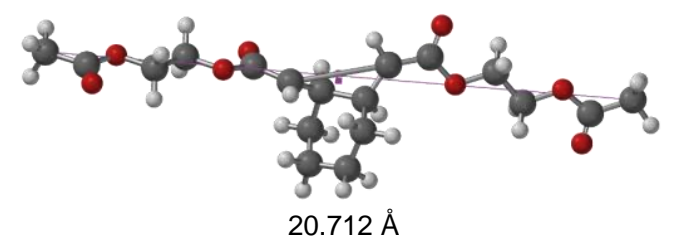

(iv) Immediately After Second Bond Cleavage

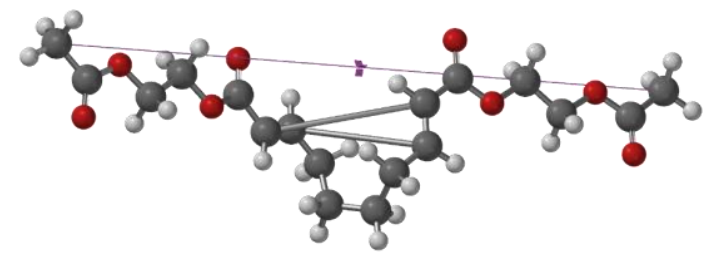

$20.762 \AA$ 
<smiles>CC(=O)OCCOC(=O)/C=C\CCCC/C=C\C(=O)OCCOC(C)=O</smiles>

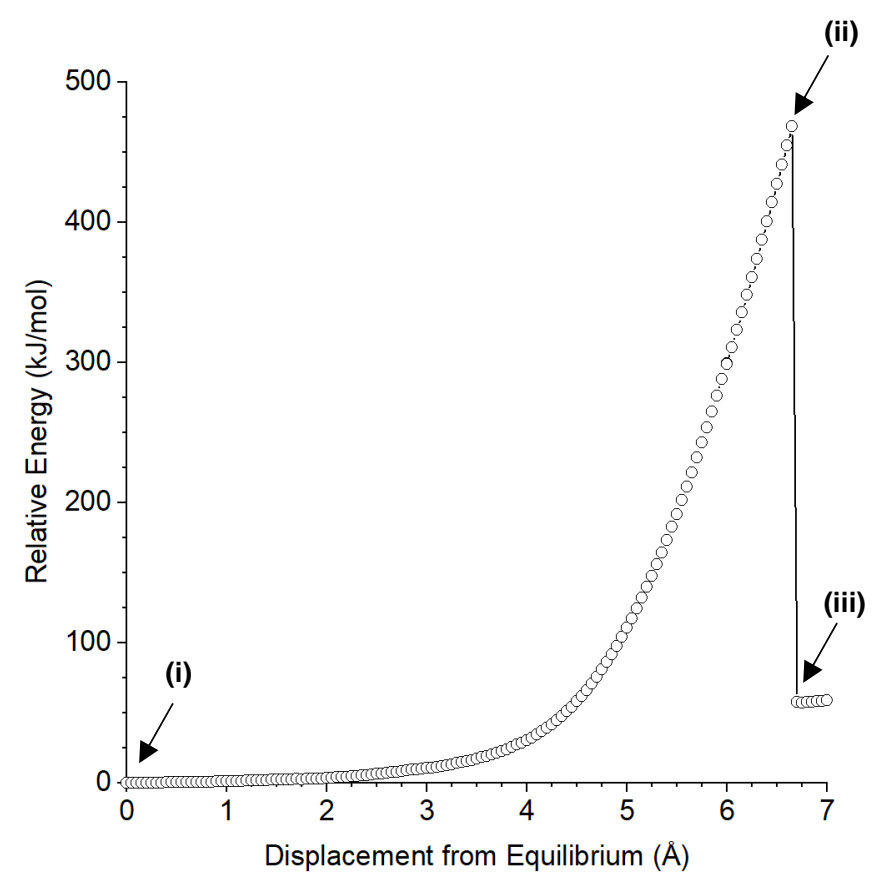

(ii)

(i) Equilibrium Geometry

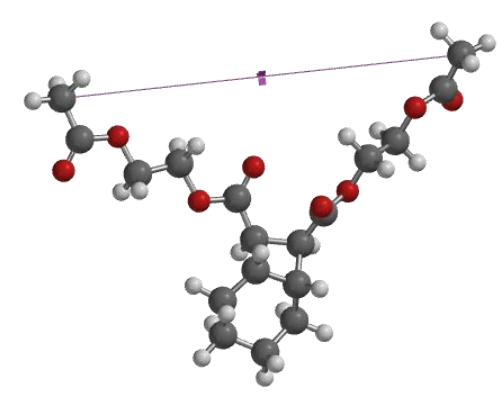

$13.678 \AA$

(ii) Immediately Prior to Bond Cleavage

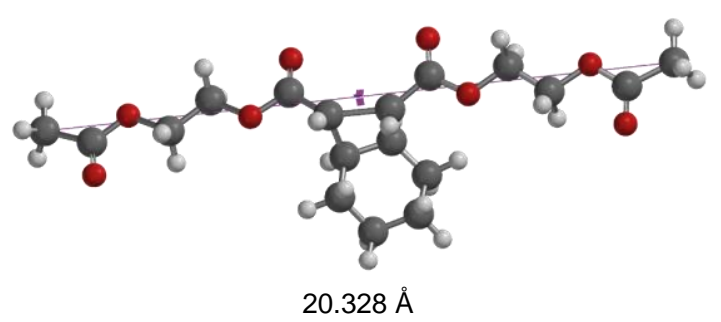

(iii) Immediately After Bond Cleavage

Summary of CoGEF Results

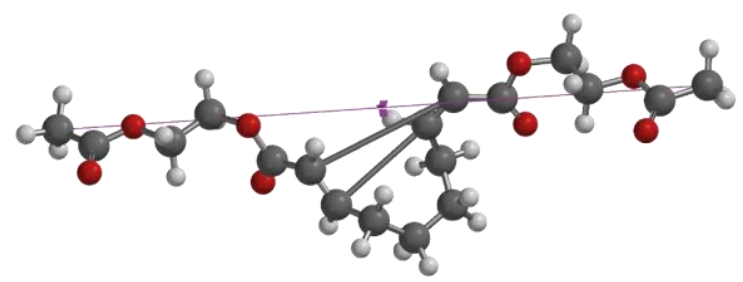

$20.378 \AA$ 

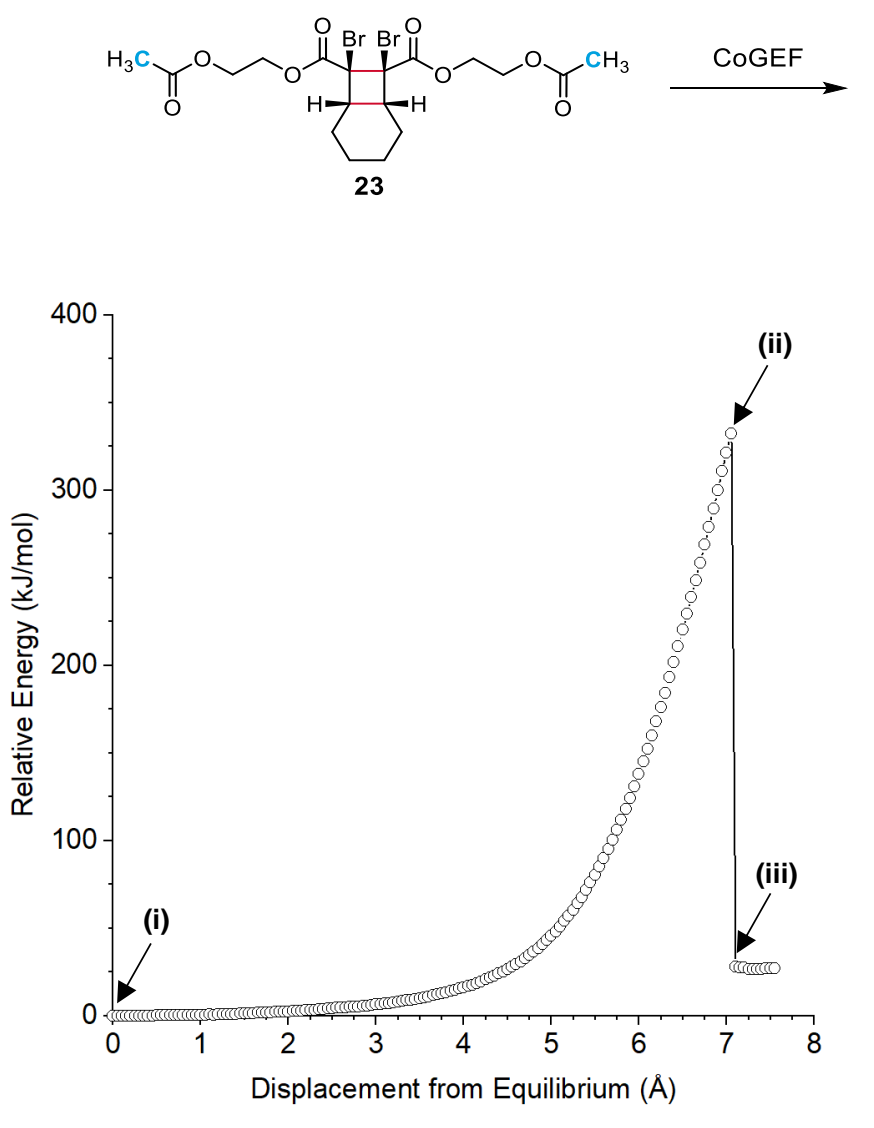

\begin{aligned} & \hline Summary of CoGEF Results \\ & \hline $\boldsymbol{F}_{\max } 3.6 \mathrm{nN} \\ & \boldsymbol{E}_{\max } 332 \mathrm{~kJ} / \mathrm{mol} \\ &$ Force-Bond Angle $6.2^{\circ}\end{aligned}$<smiles>CC(=O)OCCOC(=O)/C(Br)=C\CCCC/C=C(/Br)C(=O)OCCOC(C)=O</smiles>

(i) Equilibrium Geometry

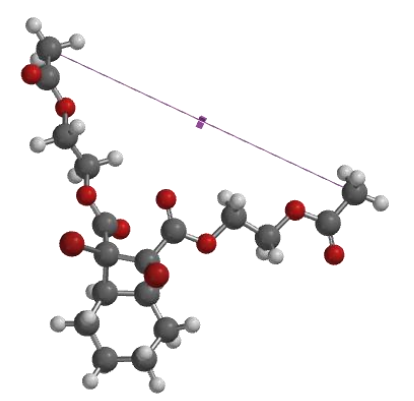

$12.604 \AA$

(ii) Immediately Prior to First Bond Cleavage

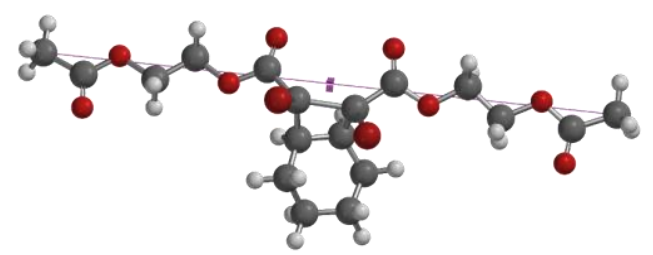

$19.654 \AA$

(iii) Immediately After First Bond Cleavage

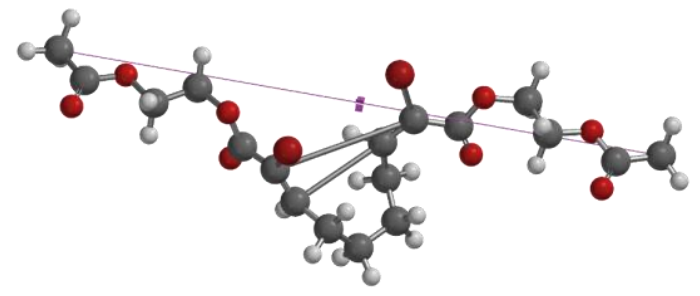

$19.704 \AA$ 


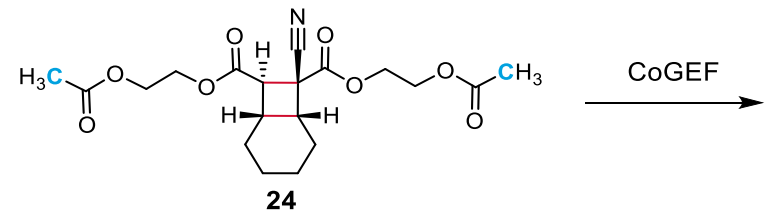<smiles>CC(=O)OCCOC(=O)/C=C\CCCC/C=C(\C#N)C(=O)OCCOC(C)=O</smiles>

(i) Equilibrium Geometry
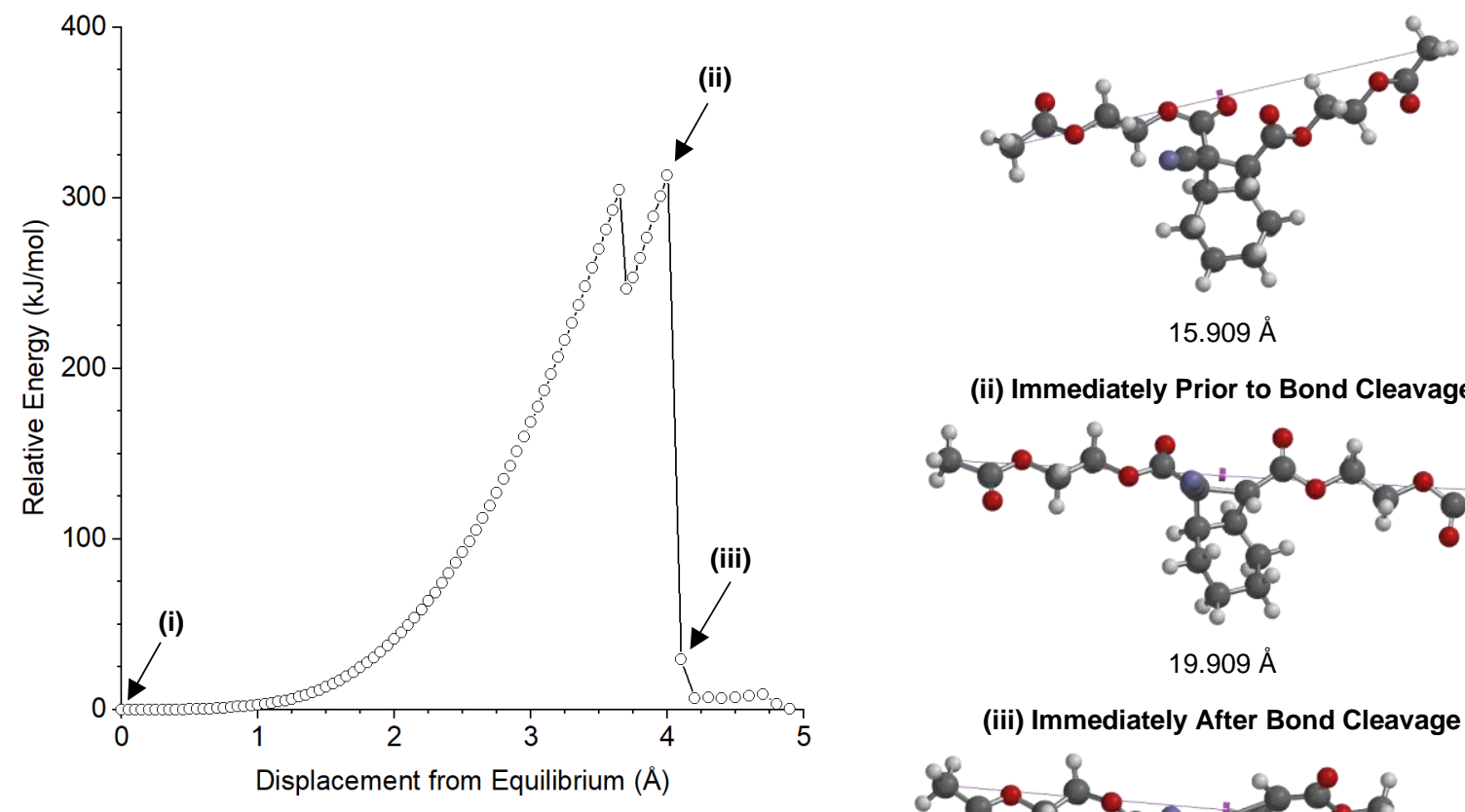

$15.909 \AA$

(ii) Immediately Prior to Bond Cleavage

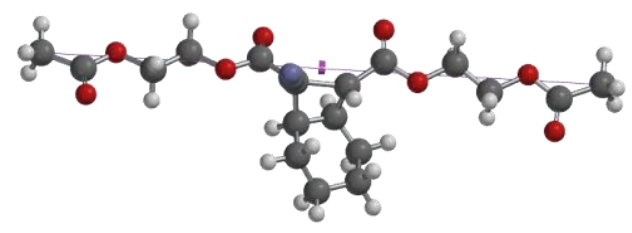

$19.909 \AA$

(iii) Immediately After Bond Cleavage

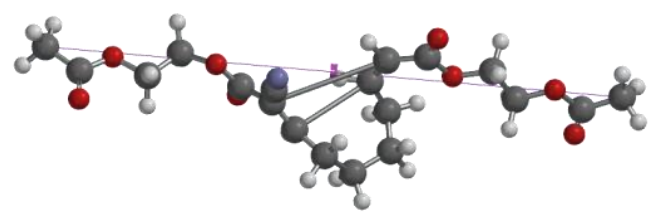

Summary of CoGEF Results

$20.009 \AA$

$\boldsymbol{F}_{\max } 4.0 \mathrm{nN}$

$E_{\max } 313 \mathrm{~kJ} / \mathrm{mol}$

Force-Bond Angle $8.5^{\circ}$ 

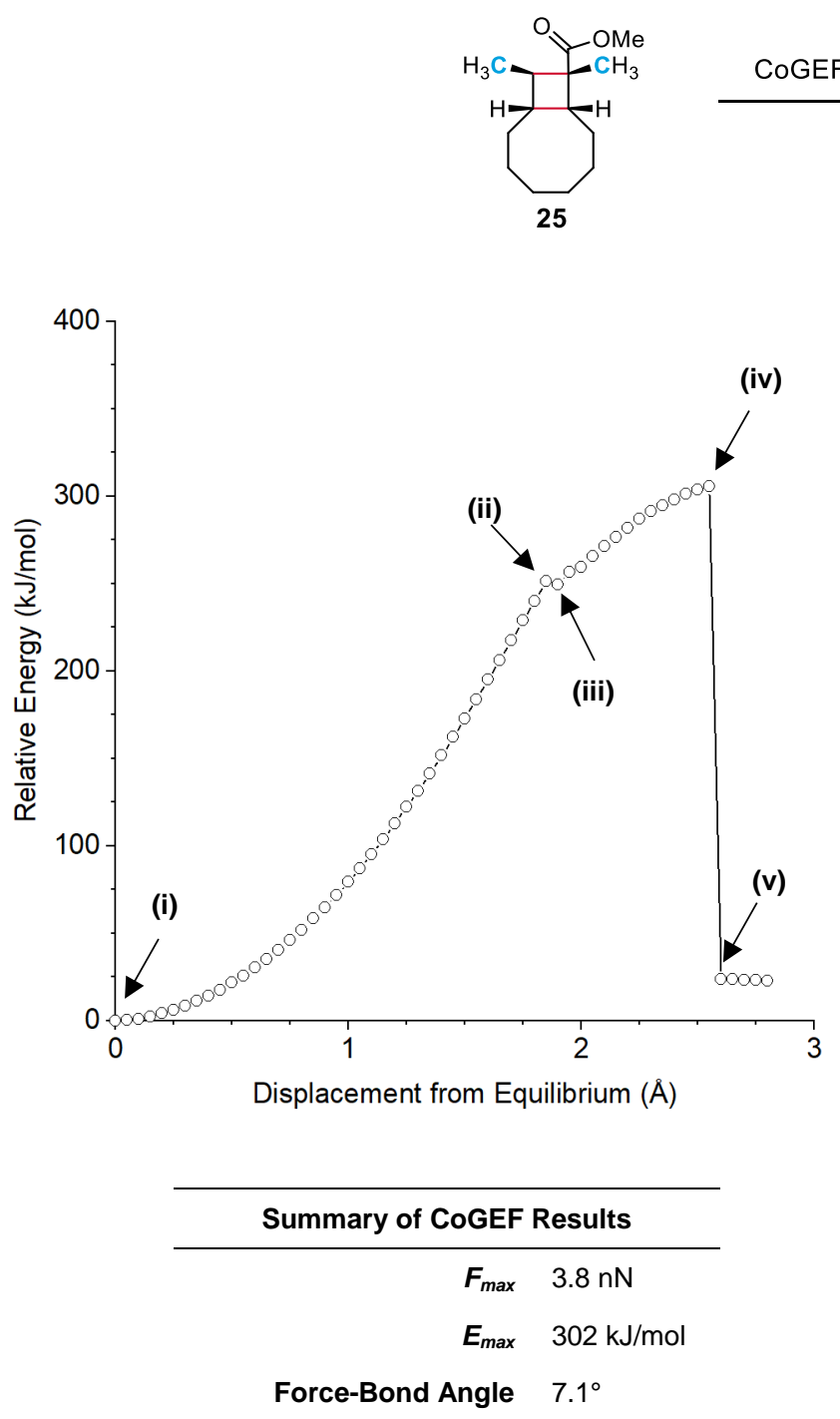

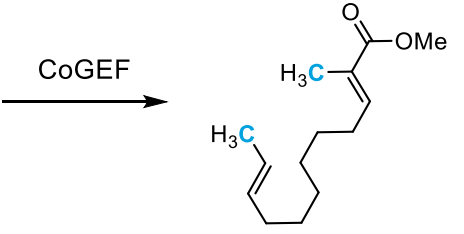

(i) Equilibrium Geometry

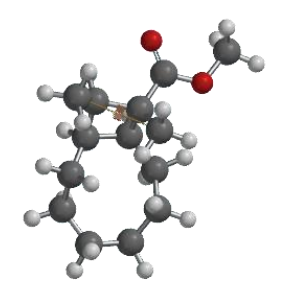

$3.052 \AA$

(ii) Immediately Prior to First Bond Cleavage

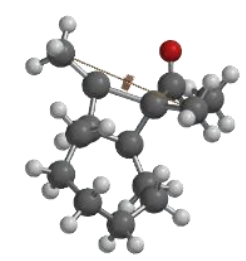

$4.902 \AA$

(iii) Immediately After First Bond Cleavage

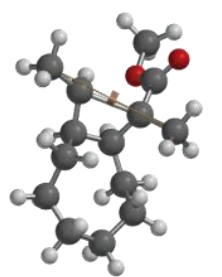

$4.952 \AA$

(iv) Immediately Prior to Second Bond Cleavage

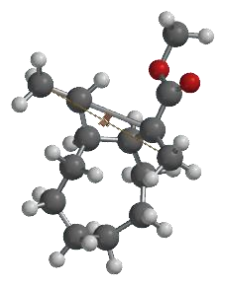

$5.552 \AA$

(v) Immediately After Second Bond Cleavage

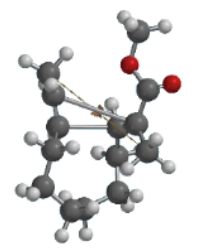

$5.602 \AA$ 


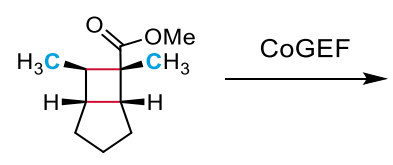

26

(ii)

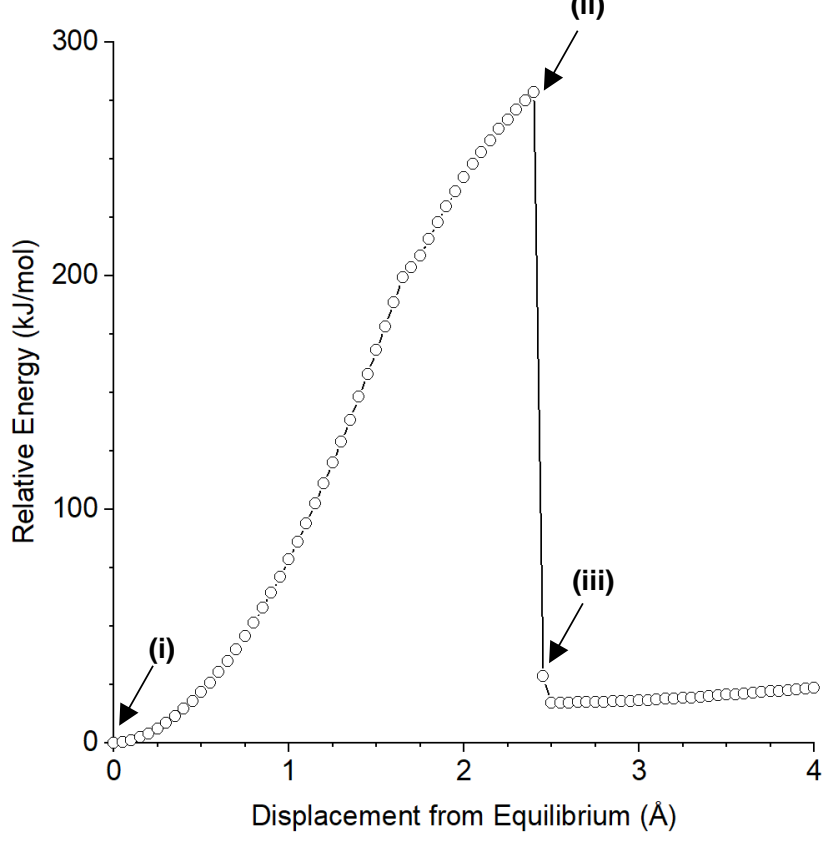

Summary of CoGEF Results

$\boldsymbol{F}_{\text {max }} \quad 3.5 \mathrm{nN}$

$E_{\max } 278 \mathrm{~kJ} / \mathrm{mol}$

Force-Bond Angle $3.1^{\circ}$

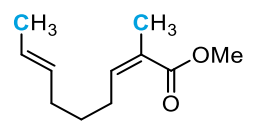

(i) Equilibrium Geometry

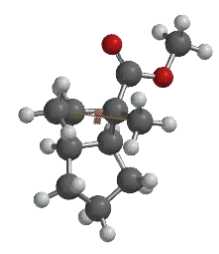

$3.057 \AA$

(ii) Immediately Prior to Bond Cleavage

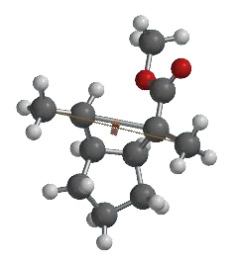

$5.457 \AA$

(iii) Immediately After Bond Cleavage

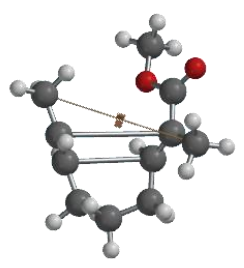

$5.507 \AA$ 


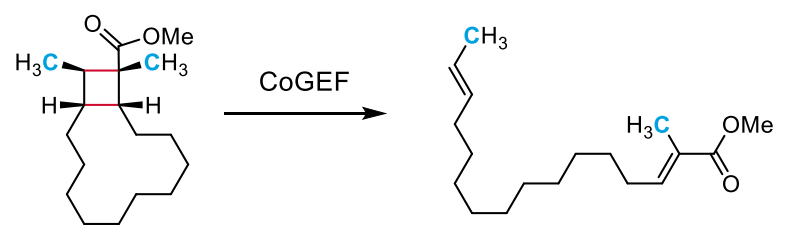

27

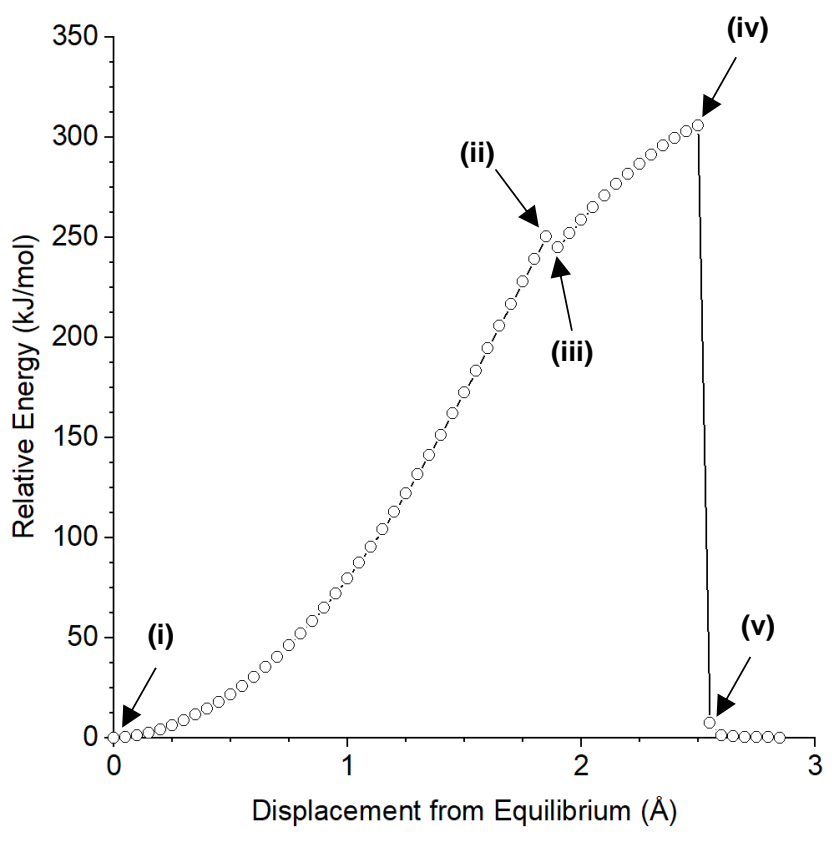

Summary of CoGEF Results

$F_{\max } \quad 3.7 \mathrm{nN}$

$E_{\max } \quad 306 \mathrm{~kJ} / \mathrm{mol}$

Force-Bond Angle $7.3^{\circ}$ (i) Equilibrium Geometry

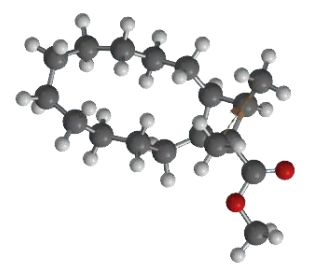

$3.051 \AA$

(ii) Immediately Prior to First Bond Cleavage

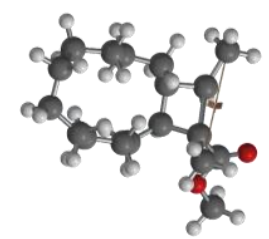

$4.901 \AA$

(iii) Immediately After First Bond Cleavage

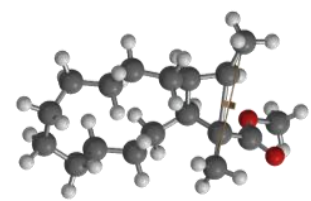

$4.951 \AA$

(iv) Immediately Prior to Second Bond Cleavage

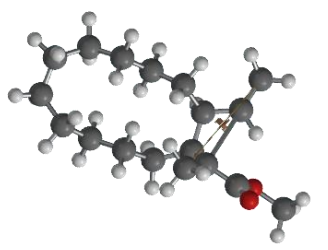

$5.551 \AA$

(v) Immediately After Second Bond Cleavage

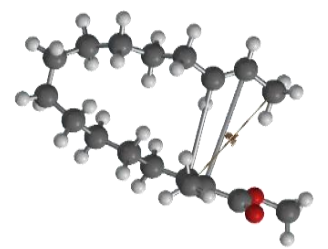

$5.601 \AA$ 

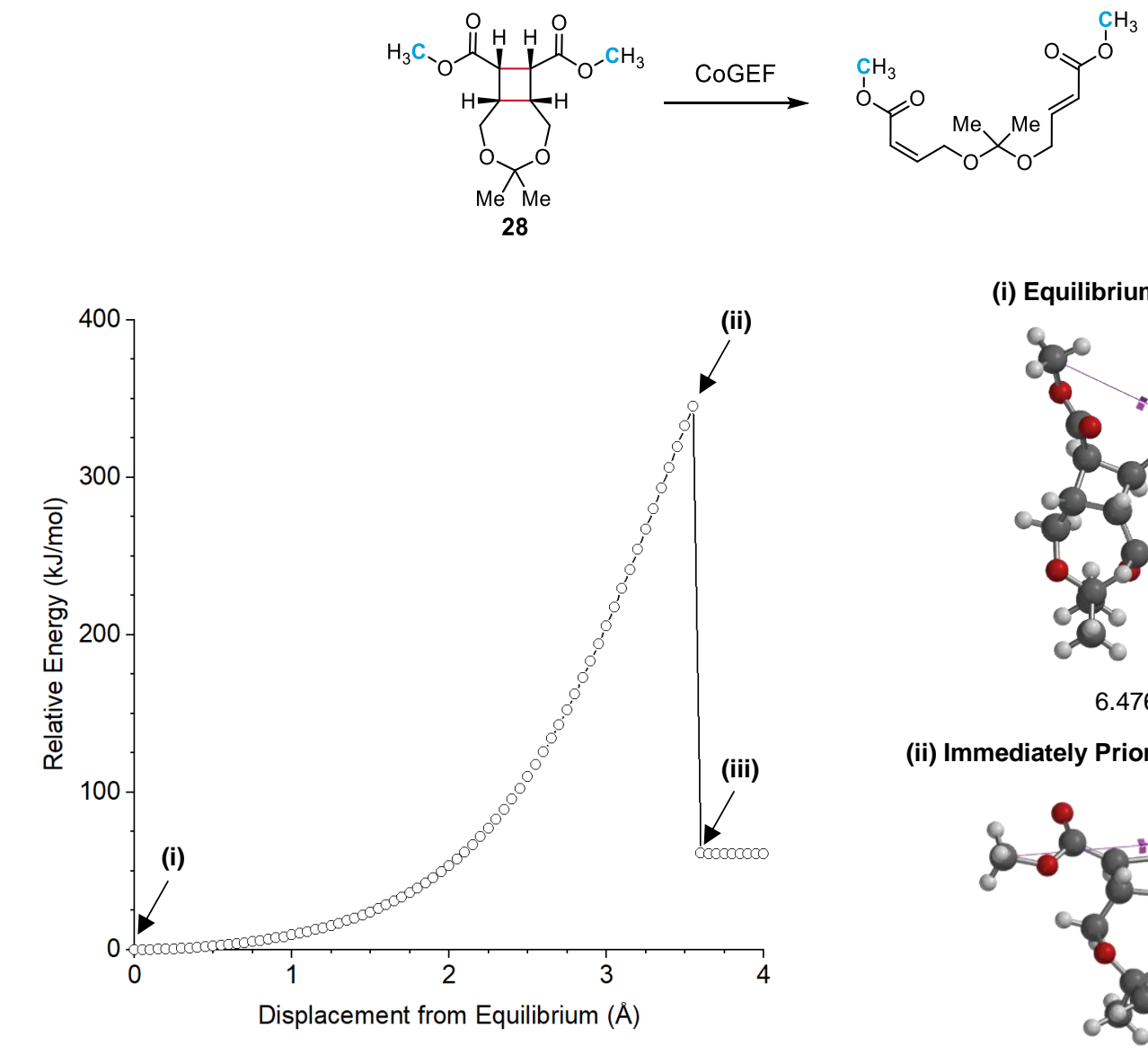

(i) Equilibrium Geometry

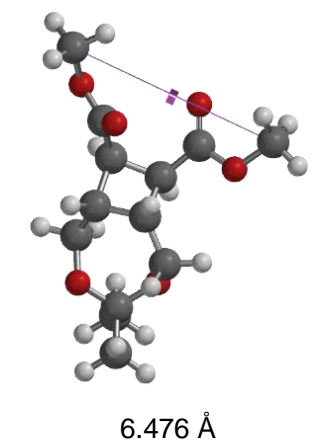

(ii) Immediately Prior to Bond Cleavage

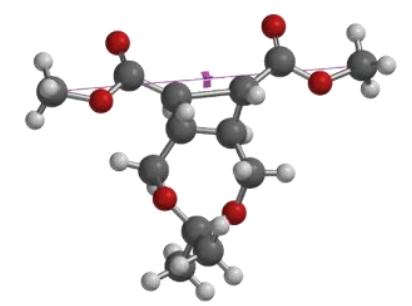

$10.026 \AA$

(iii) Immediately After Bond Cleavage

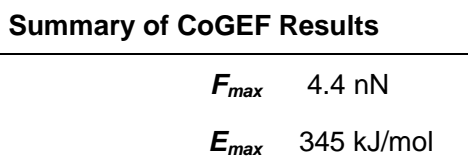

Force-Bond Angle $4.6^{\circ}$

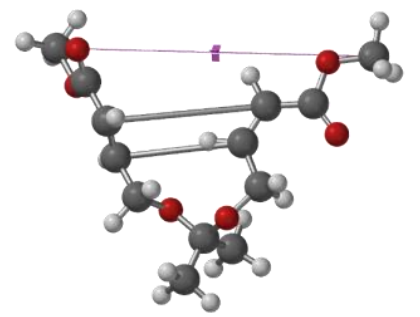

$10.076 \AA$ 


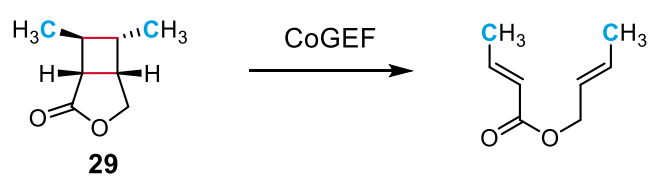

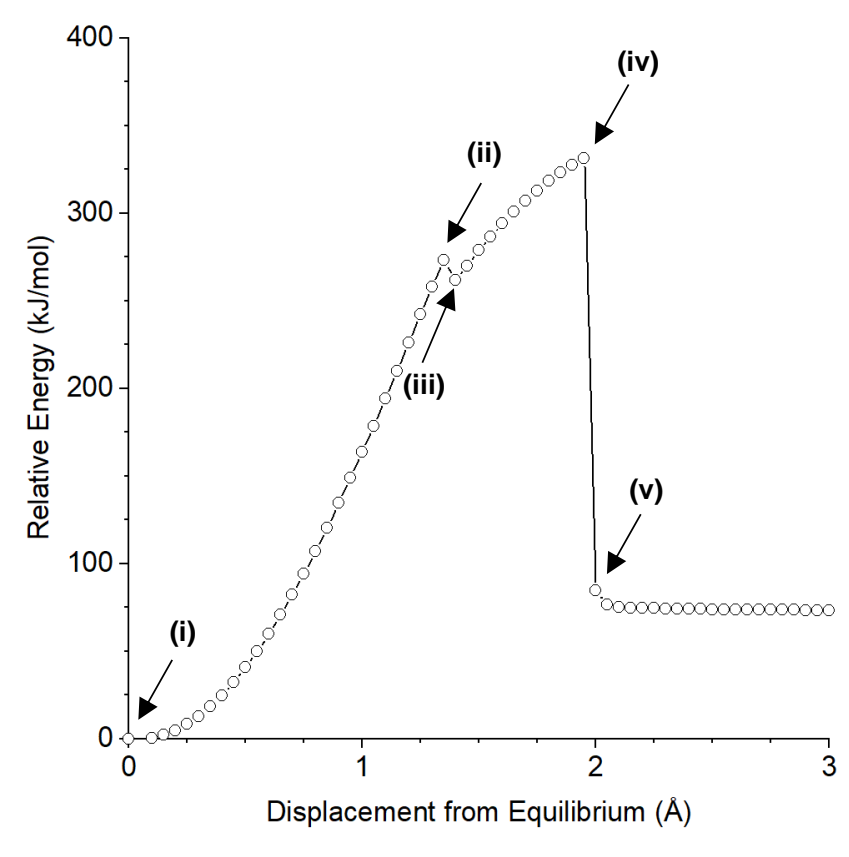

Summary of CoGEF Results

$$
\begin{array}{ll}
\boldsymbol{F}_{\text {max }} & 5.4 \mathrm{nN} \\
\boldsymbol{E}_{\max } & 331 \mathrm{~kJ} / \mathrm{mol}
\end{array}
$$

Force-Bond Angle $24^{\circ}$ (i) Equilibrium Geometry

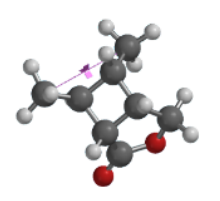

$3.769 \AA$

(ii) Immediately Prior to First Bond Cleavage

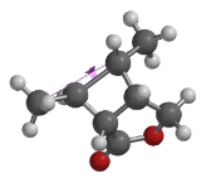

$5.119 \AA$

(iii) Immediately After First Bond Cleavage

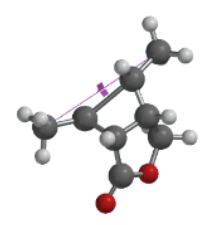

$5.169 \AA$

(iv) Immediately Prior to Second Bond Cleavage

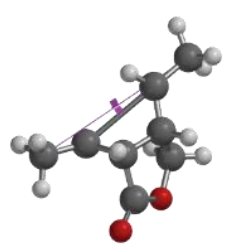

$5.719 \AA$

(v) Immediately After Second Bond Cleavage

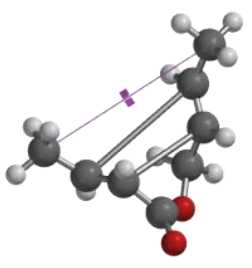

$5.769 \AA$ 

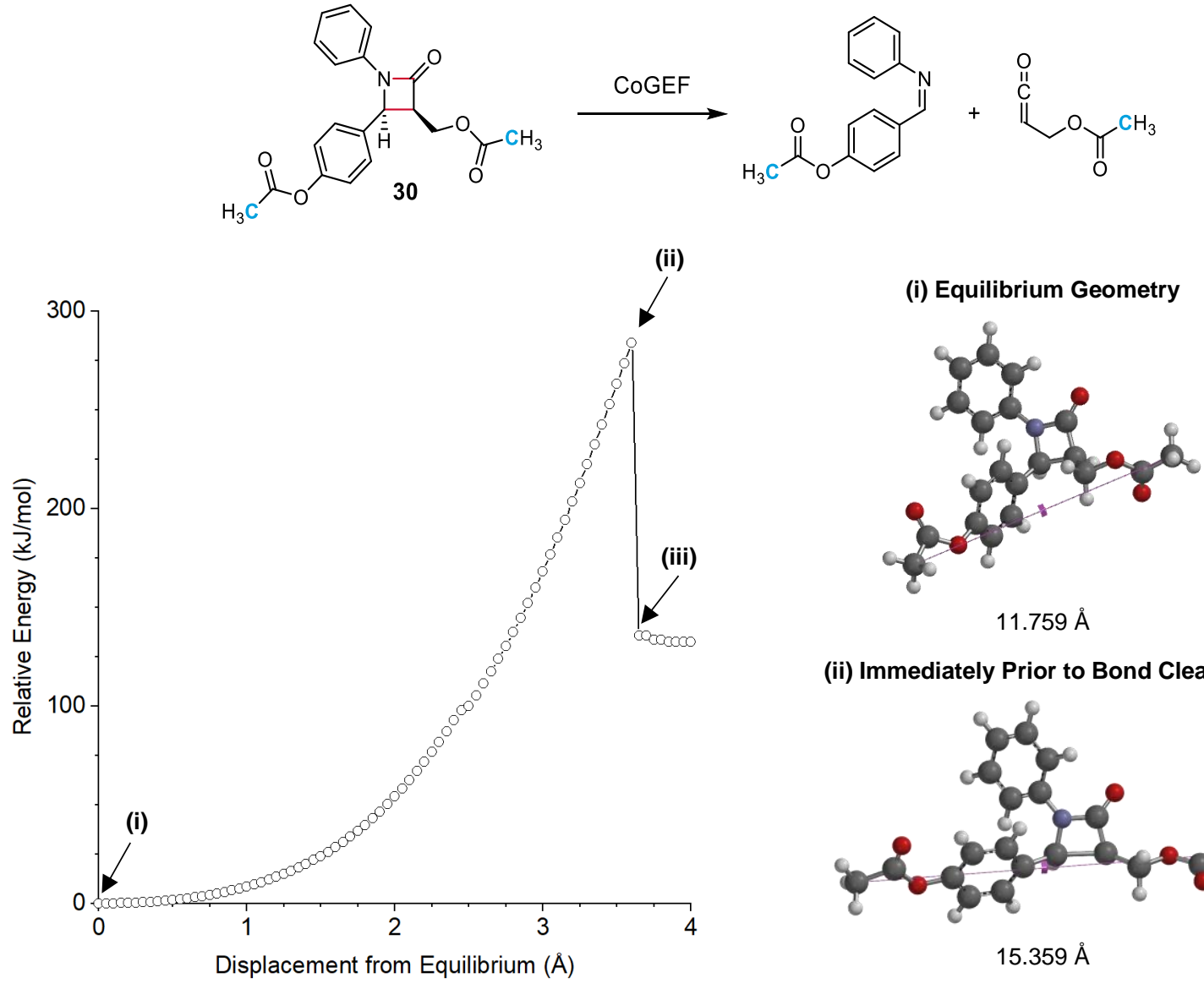

(ii) Immediately Prior to Bond Cleavage

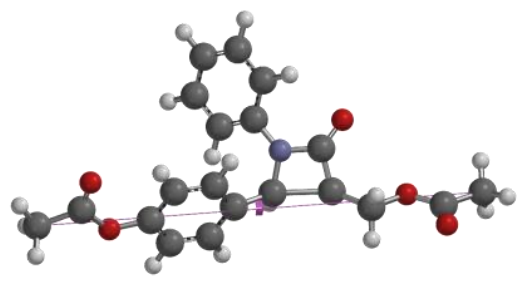

$15.359 \AA$

(iii) Immediately After Bond Cleavage

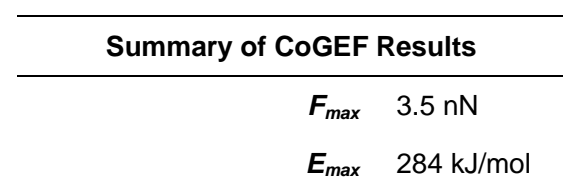

Force-Bond Angle $9.3^{\circ}$

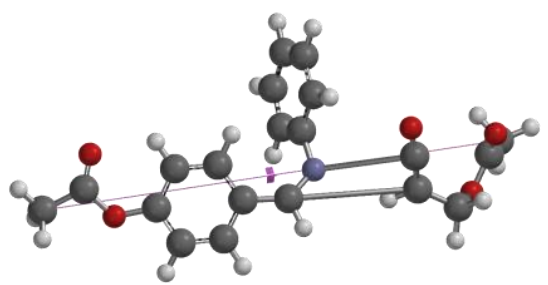

$15.409 \AA$ 
<smiles>CC(=O)OCCOc1ccc(N2C(=O)C(c3ccccc3)(c3ccccc3)N2c2ccc(OCCOC(C)=O)cc2)cc1</smiles><smiles>CC(=O)OCCOc1ccc(N=C=O)cc1</smiles>

(ii)

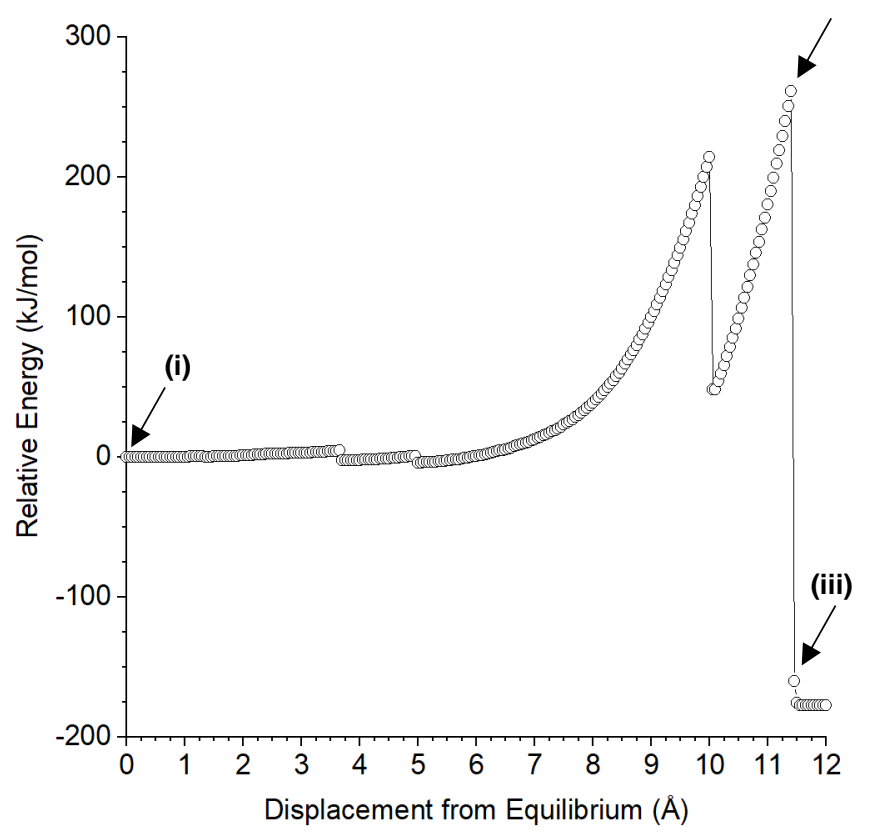

Summary of CoGEF Results

$\boldsymbol{F}_{\max } \quad 3.6 \mathrm{nN}$

$E_{\max } \quad 260 \mathrm{~kJ} / \mathrm{mol}$

Force-Bond Angle $34^{\circ}$ (i) Equilibrium Geometry

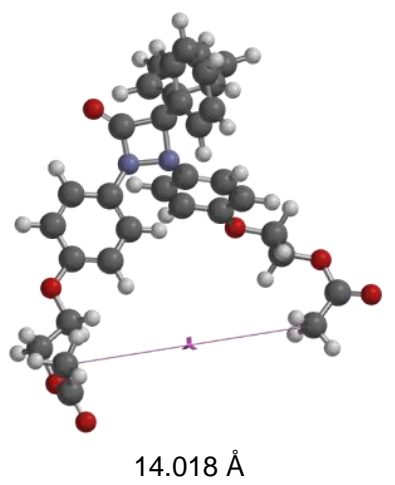

(ii) Immediately Prior to Bond Cleavage

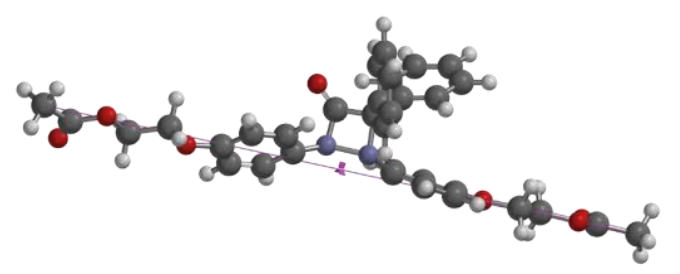

$25.418 \AA$

(iii) Immediately After Bond Cleavage

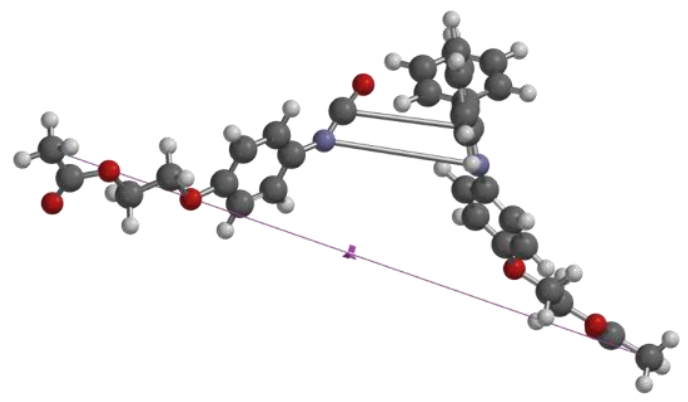

$25.468 \AA$ 

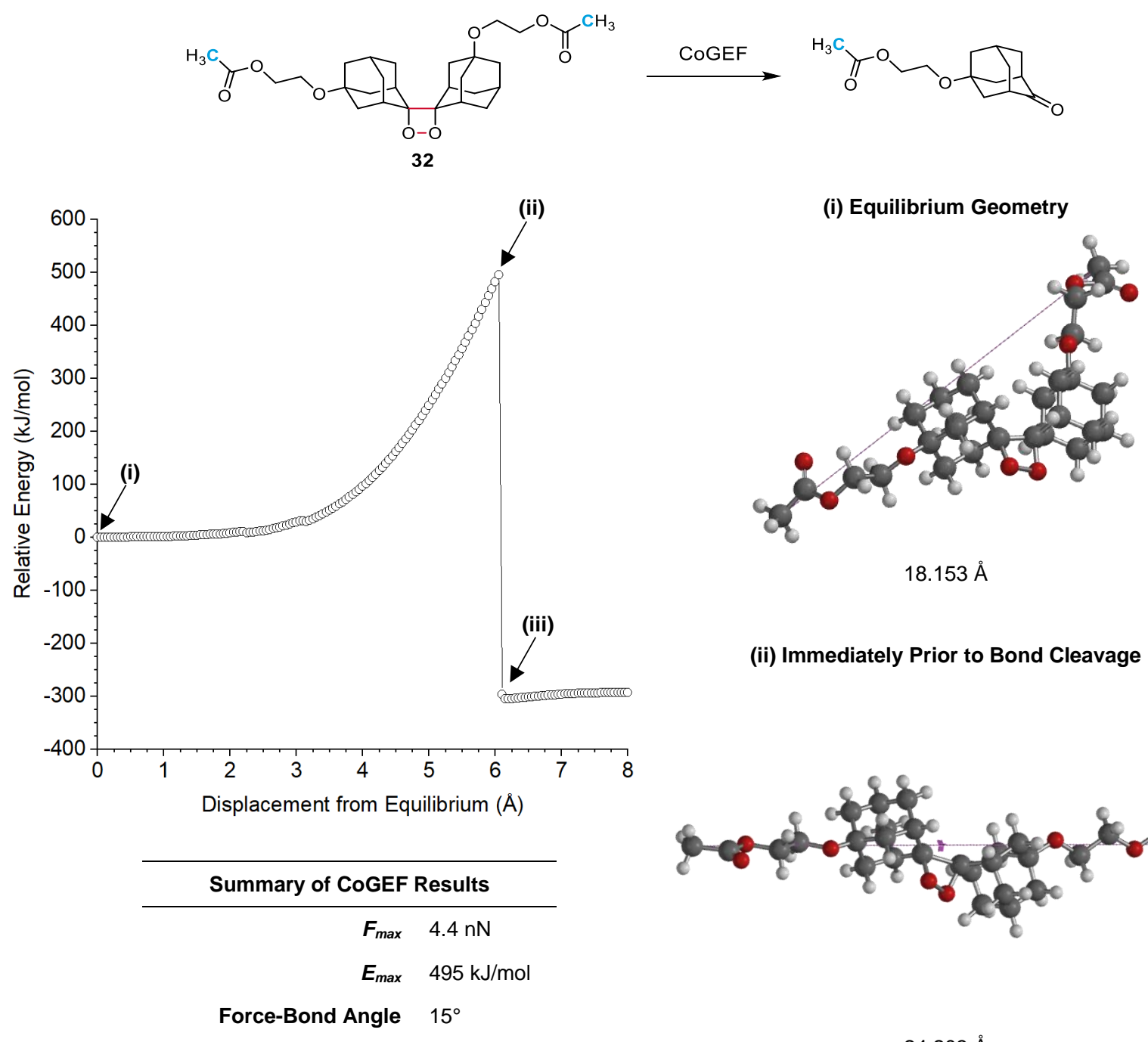

$18.153 \AA$

(ii) Immediately Prior to Bond Cleavage

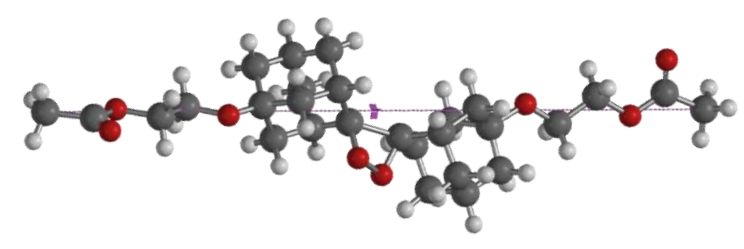

$24.203 \AA$

(iii) Immediately After Bond Cleavage

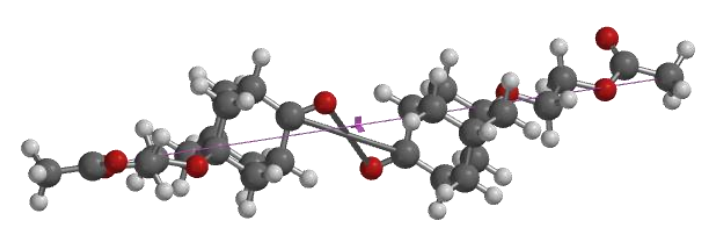

$24.253 \AA$ 

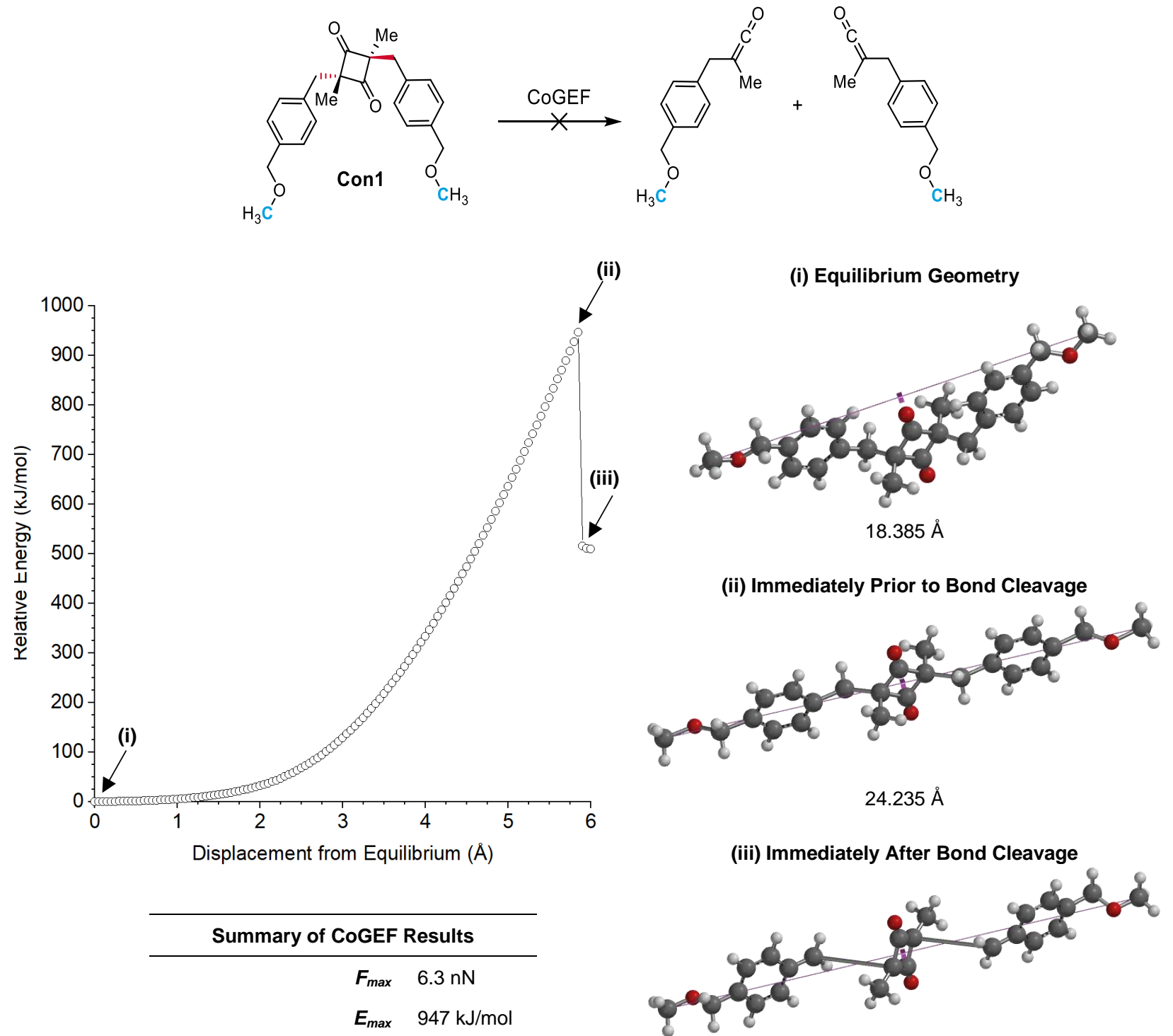

Force-Bond Angle $43^{\circ}$

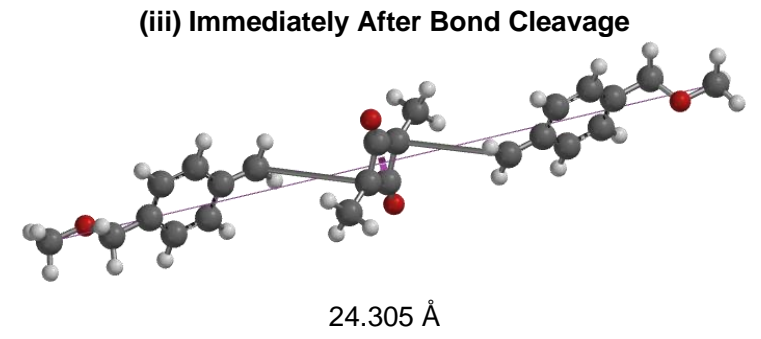



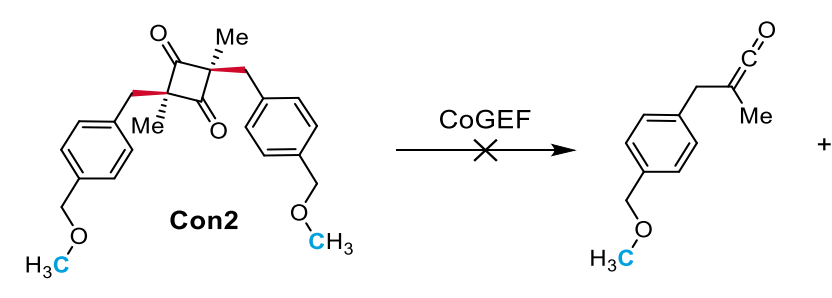

(ii)

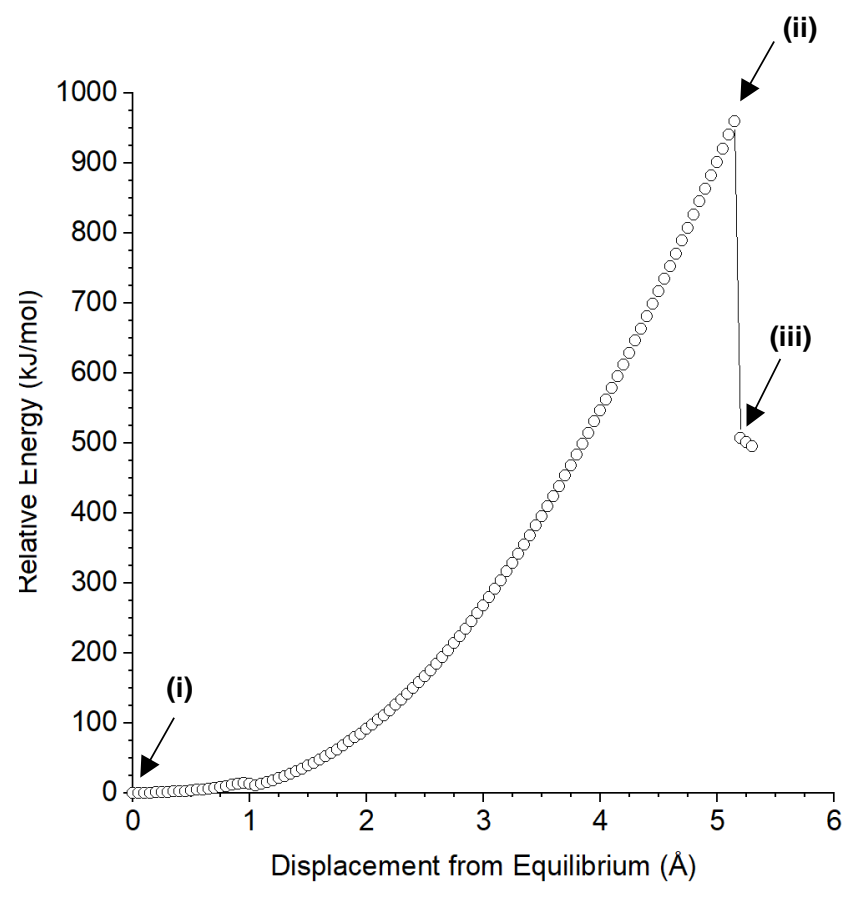

Summary of CoGEF Results

$F_{\max } \quad 6.4 \mathrm{nN}$

$E_{\max } \quad 959 \mathrm{~kJ} / \mathrm{mol}$

Force-Bond Angle $42^{\circ}$

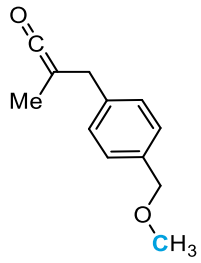

(i) Equilibrium Geometry

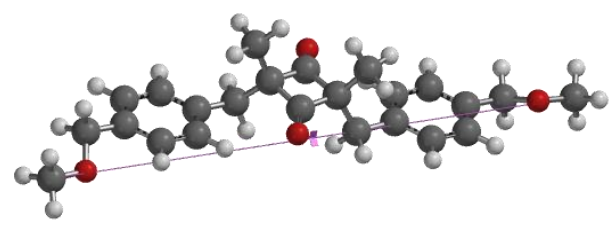

$19.350 \AA$

(ii) Immediately Prior to Bond Cleavage

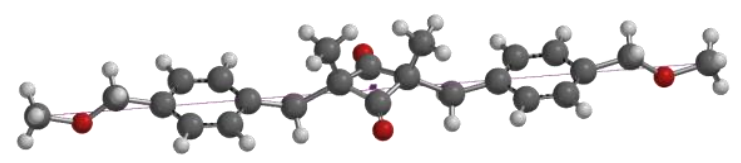

$24.200 \AA$

(iii) Immediately After Bond Cleavage

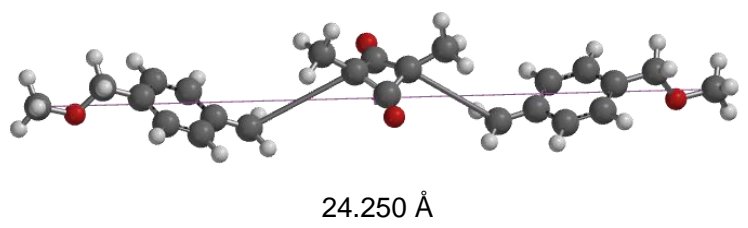




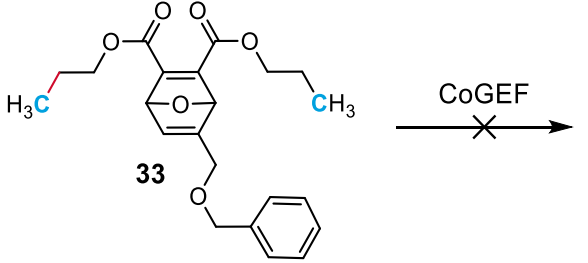

(ii)

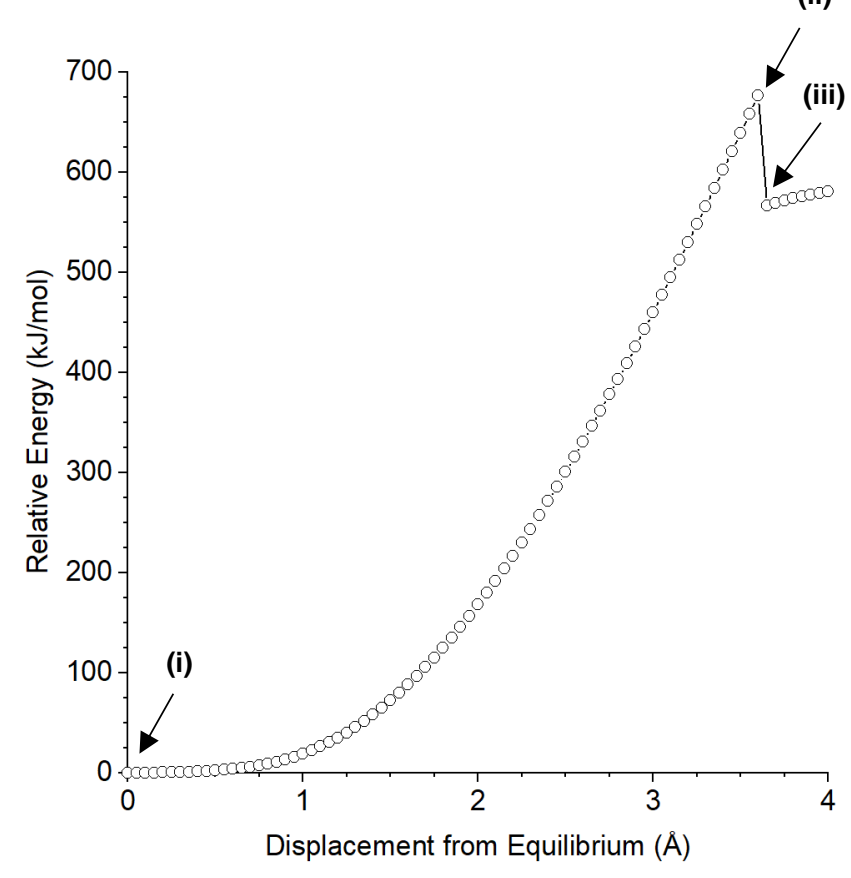

Summary of CoGEF Results

$F_{\max } \quad 6.2 \mathrm{nN}$

$E_{\max } \quad 676 \mathrm{~kJ} / \mathrm{mol}$

Force-Bond Angle $78^{\circ}$ (i) Equilibrium Geometry

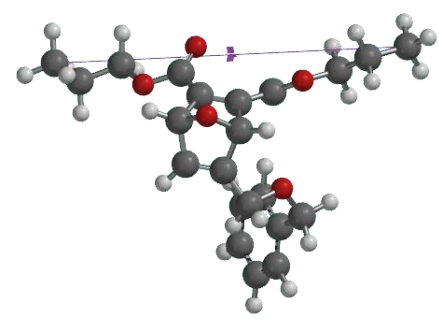

$12.509 \AA$

(ii) Immediately Prior to Bond Cleavage

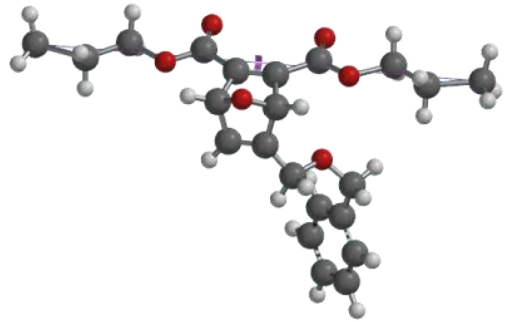

$16.109 \AA$

(iii) Immediately After Bond Cleavage

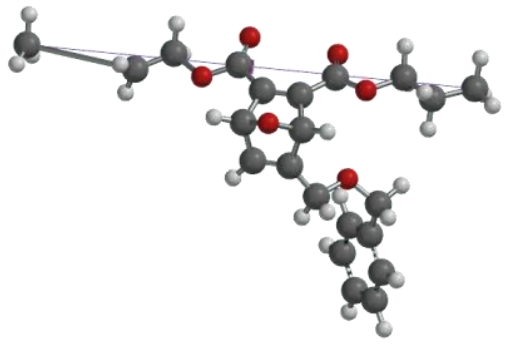

$16.159 \AA$ 

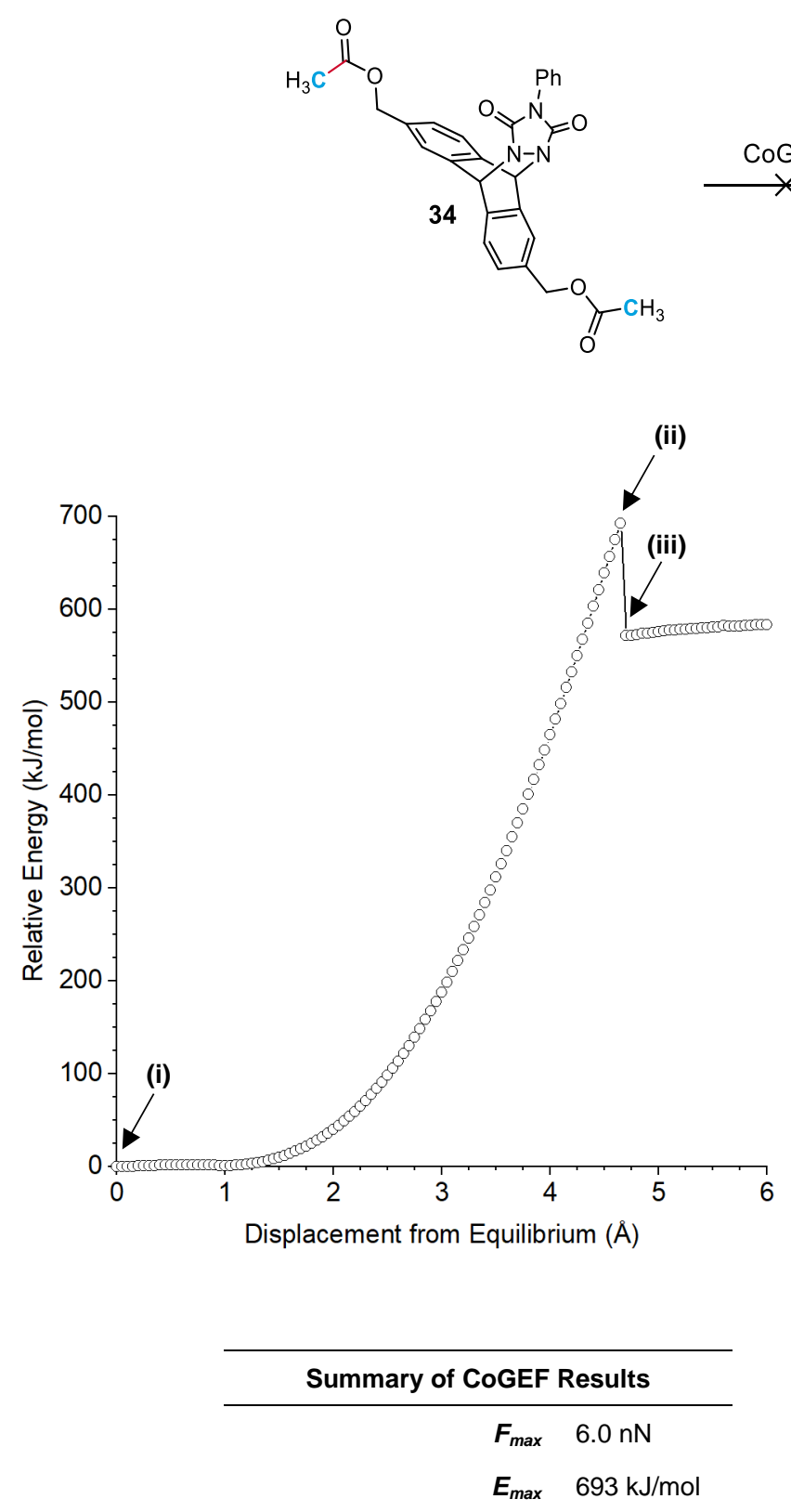

Force-Bond Angle $85^{\circ}$

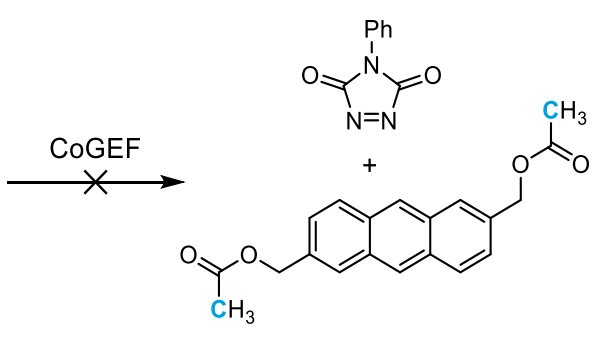

(i) Equilibrium Geometry

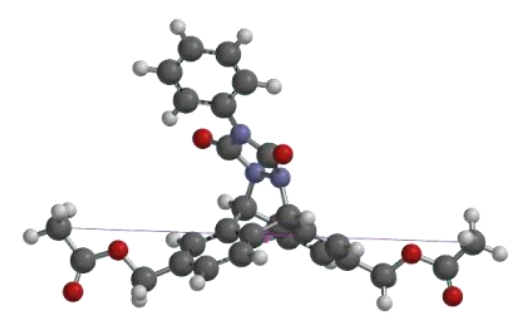

$15.358 \AA$

(ii) Immediately Prior to Bond Cleavage

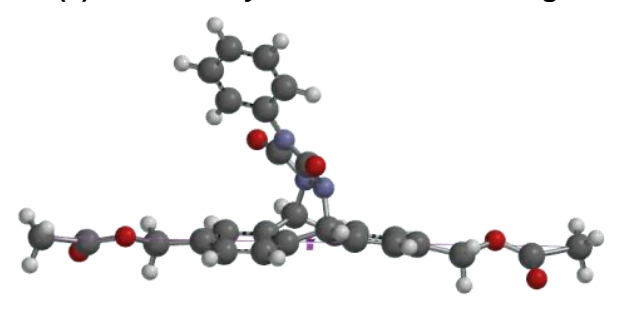

$20.008 \AA$

(iii) Immediately After Bond Cleavage

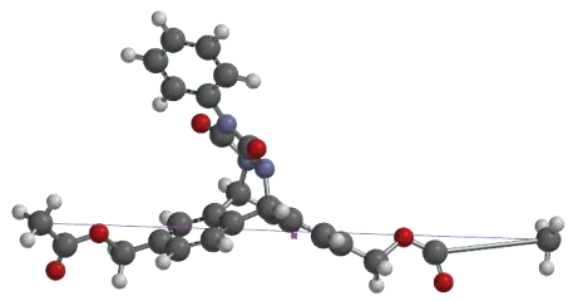

$20.058 \AA$ 


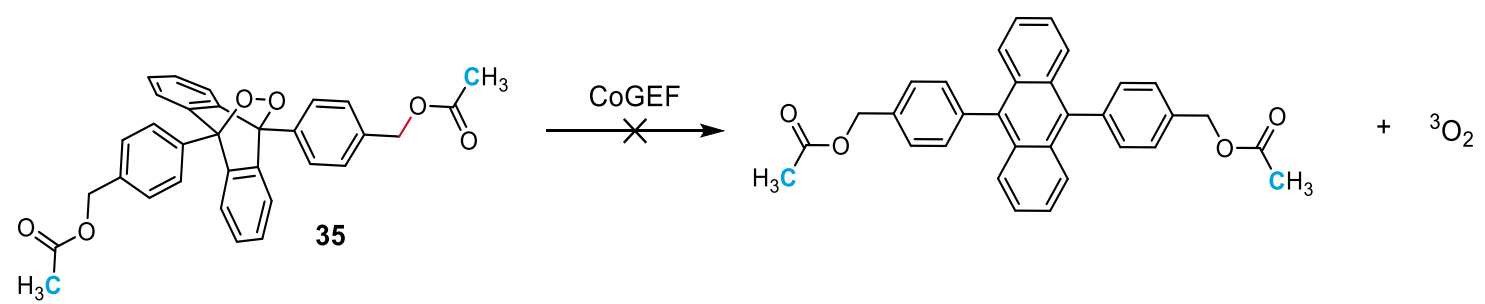

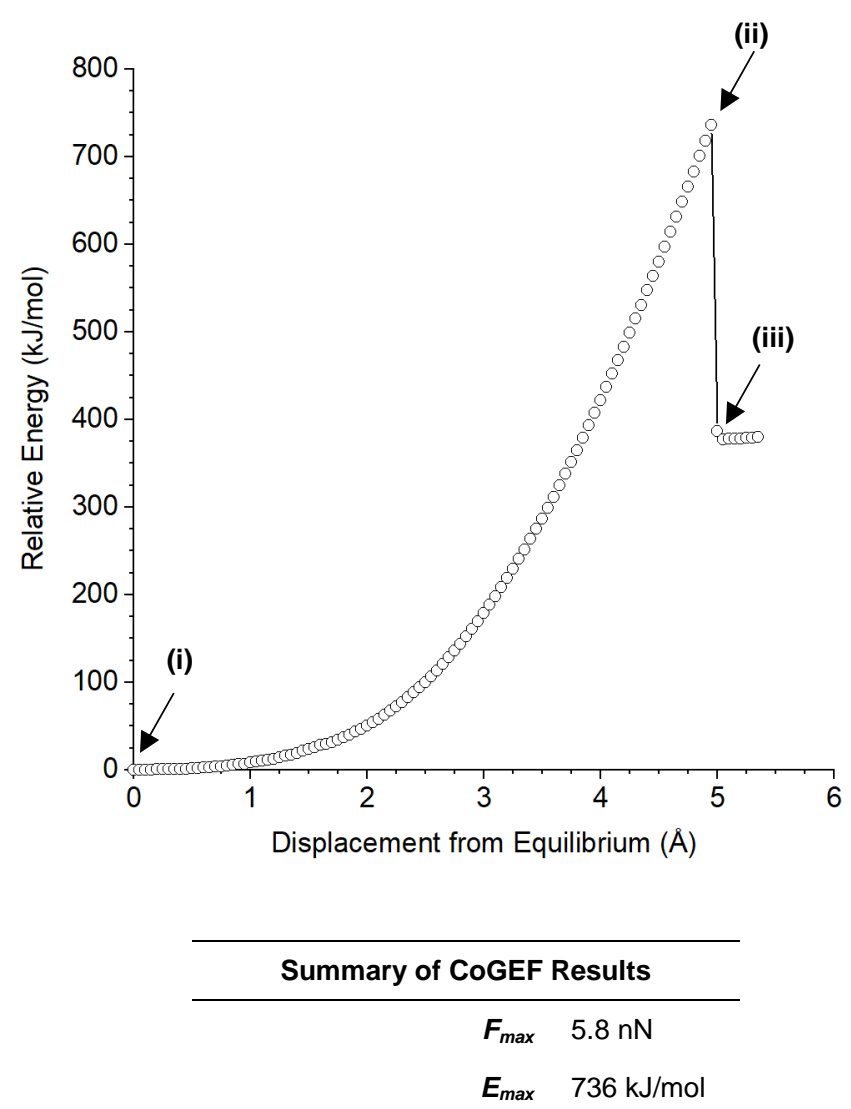

Force-Bond Angle $66^{\circ}$ (i) Equilibrium Geometry

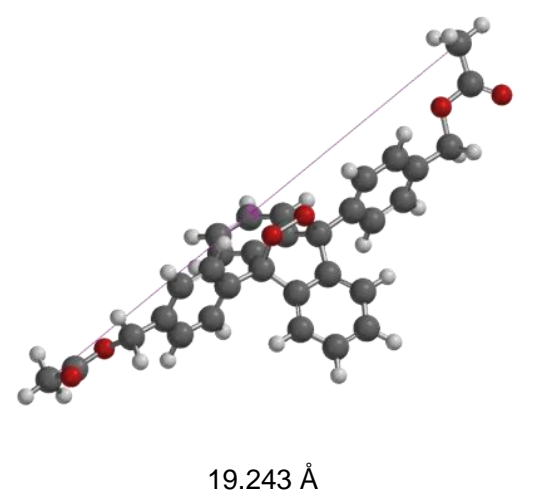

(ii) Immediately Prior to Bond Cleavage

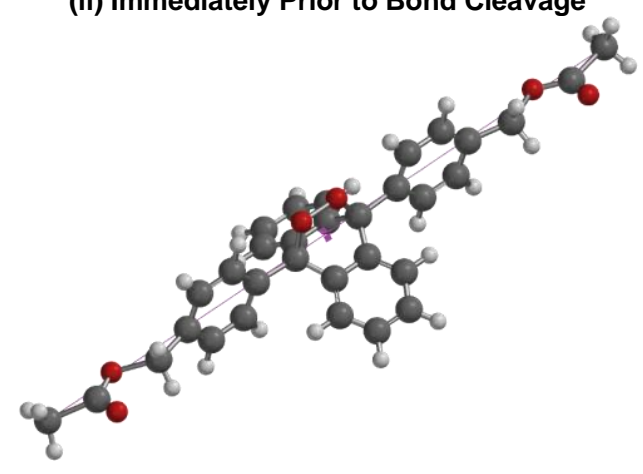

$24.193 \AA$

(iii) Immediately After Bond Cleavage

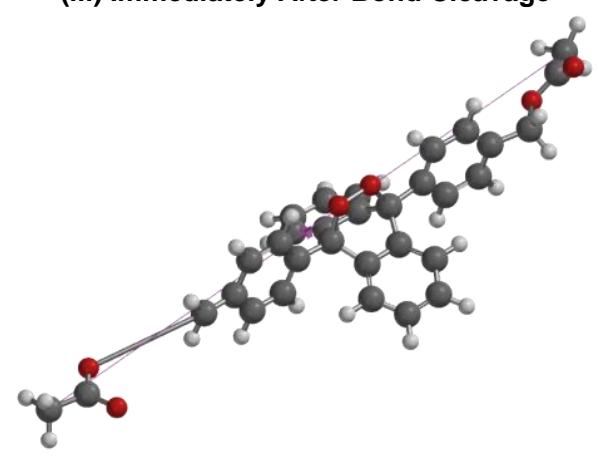

$24.243 \AA$ 
<smiles>CC(=O)OCC1C=CC(C)C(SCc2ccc(CSC3(c4ccccn4)SC(C)CC=CC3COC(C)=O)cc2)(c2ccccn2)S1</smiles>

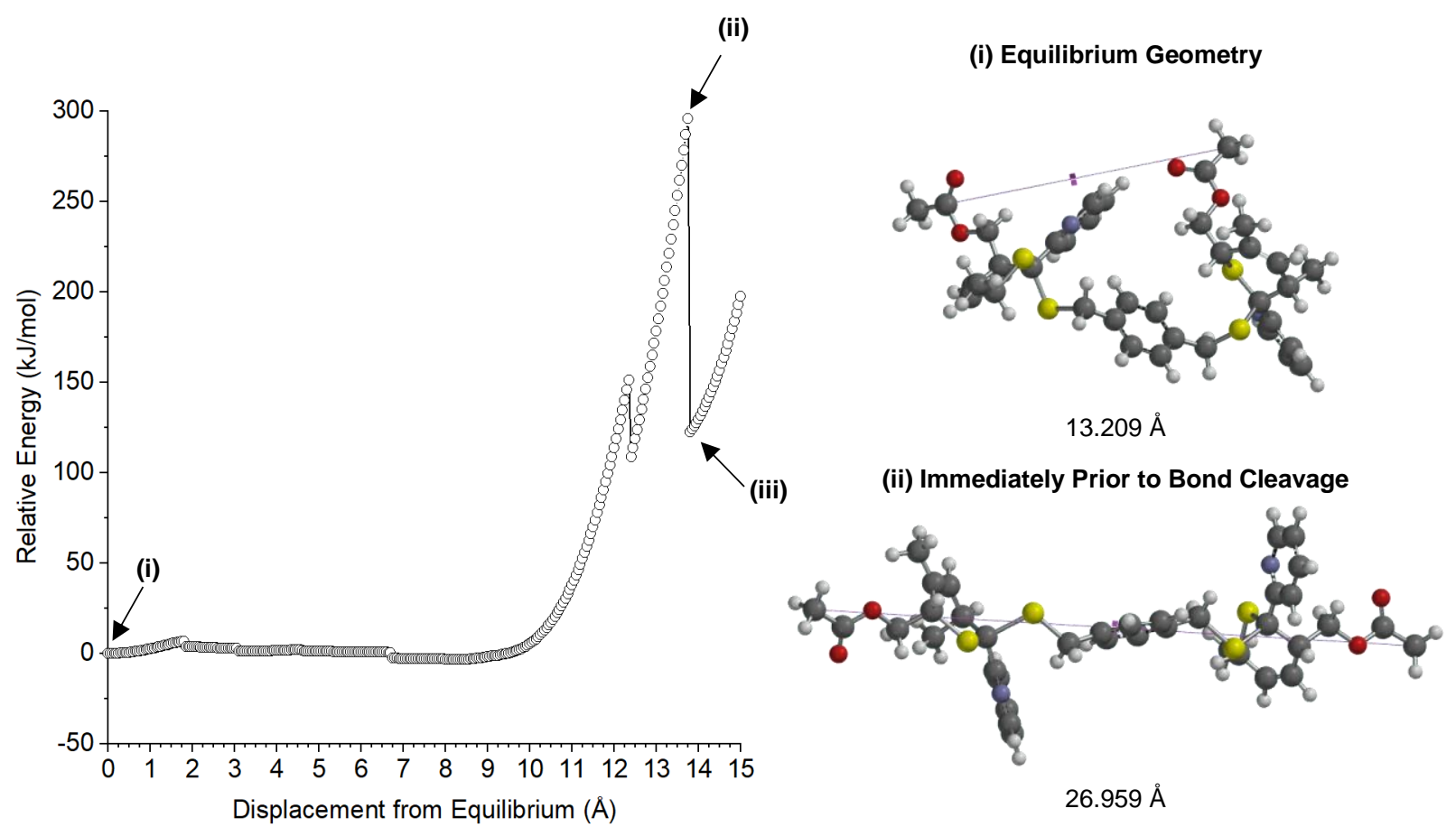

(iii) Immediately After Bond Cleavage

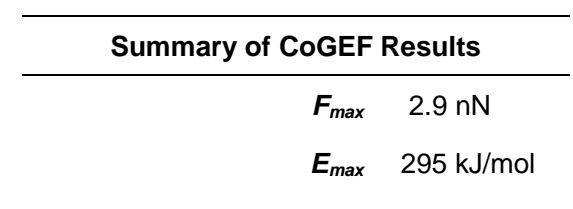

Force-Bond Angle $32^{\circ}$

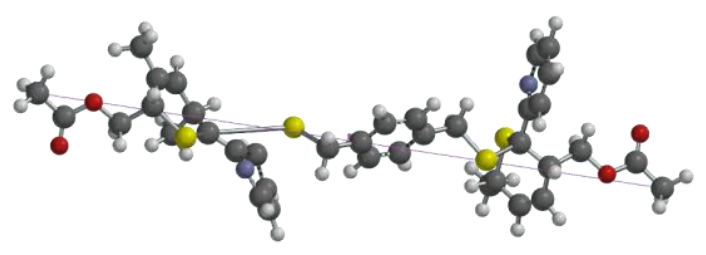

$27.009 \AA$ 

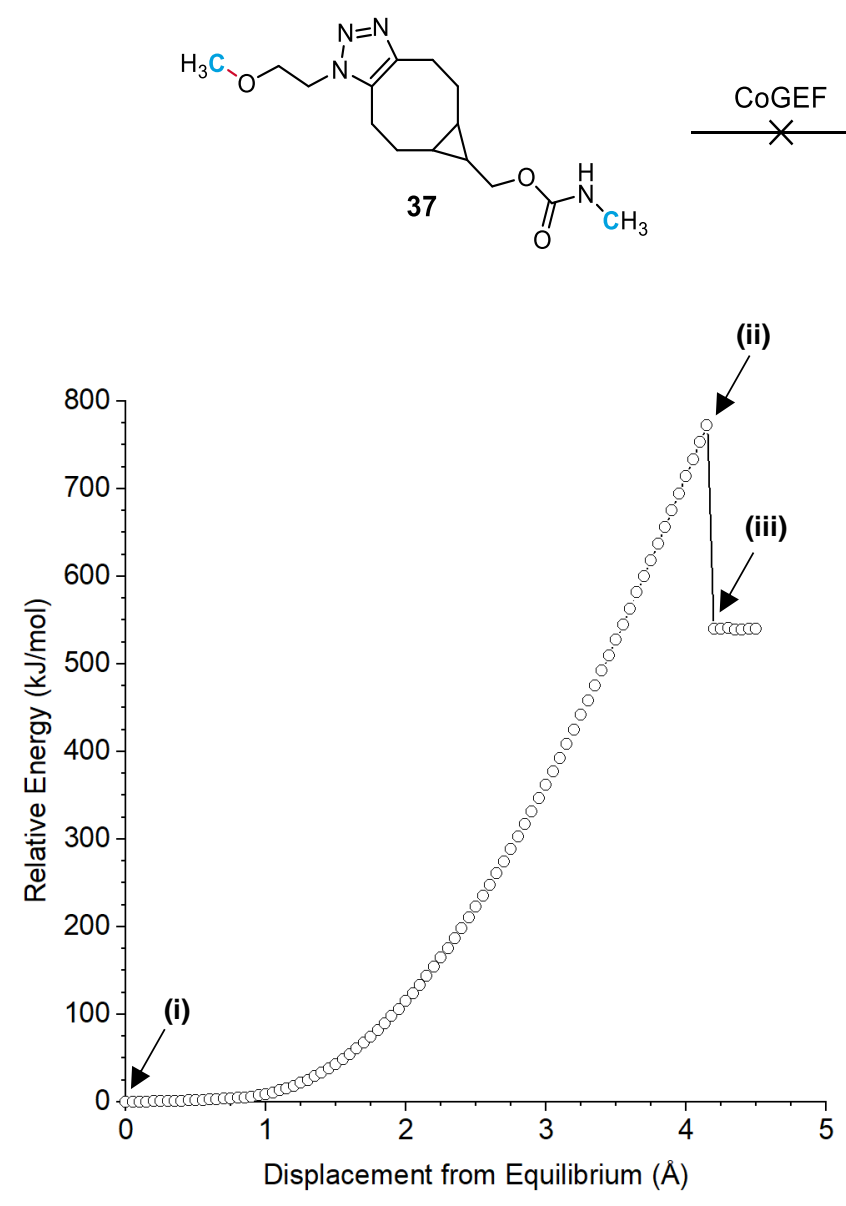

Summary of CoGEF Results

$$
\begin{array}{ll}
\boldsymbol{F}_{\text {max }} & 6.5 \mathrm{nN} \\
\boldsymbol{E}_{\text {max }} & 772 \mathrm{~kJ} / \mathrm{mol}
\end{array}
$$

Force/Bond angle $8.9^{\circ}$ (i) Equilibrium Geometry

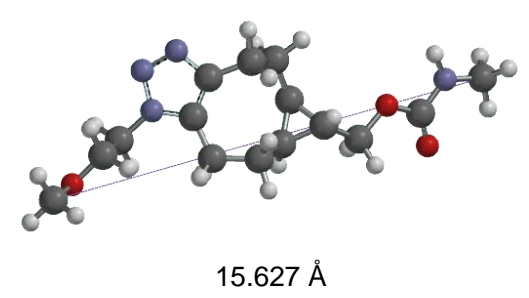

(ii) Immediately Prior to Bond Cleavage

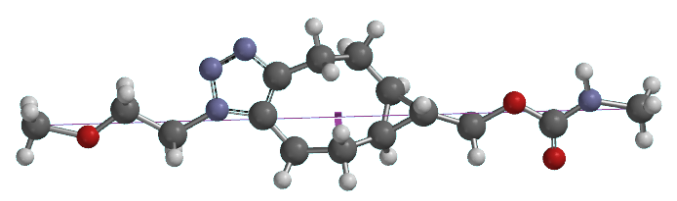

$19.777 \AA$

(iii) Immediately After Bond Cleavage

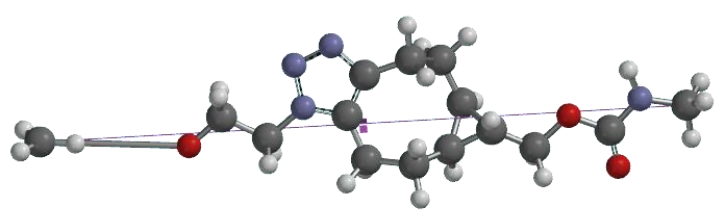

$19.827 \AA$ 


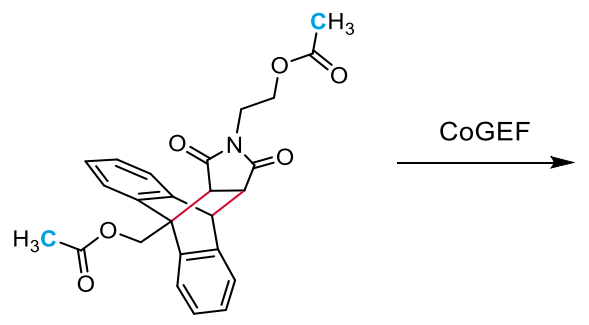

38

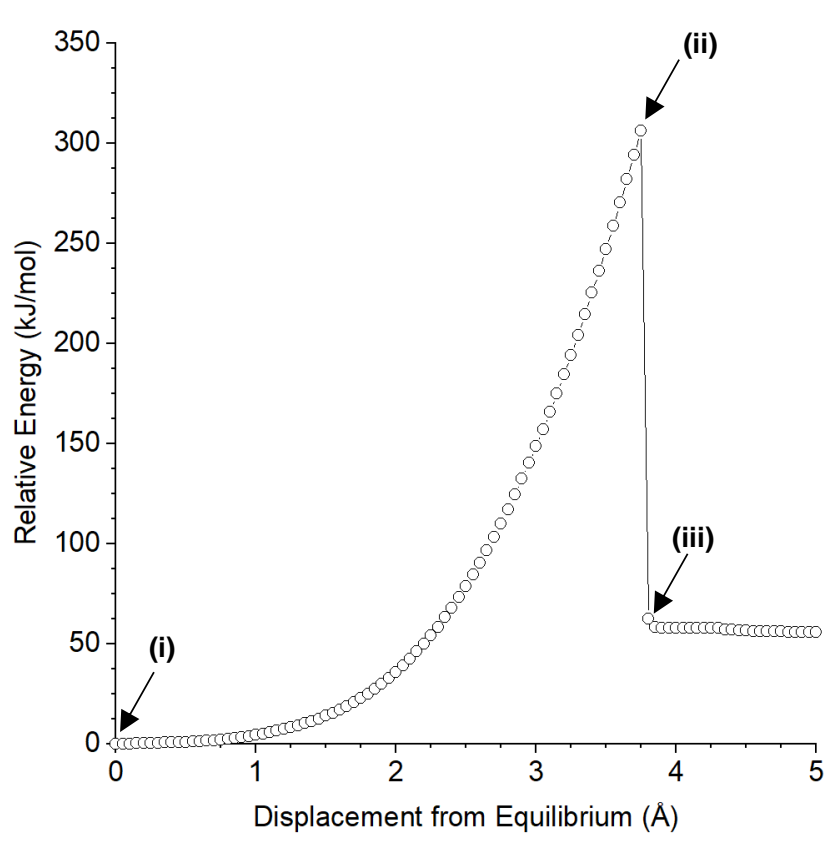

Summary of CoGEF Results

$\boldsymbol{F}_{\max } \quad 4.1 \mathrm{nN}$

$E_{\max } \quad 306 \mathrm{~kJ} / \mathrm{mol}$

Force/Bond angle $25^{\circ}$

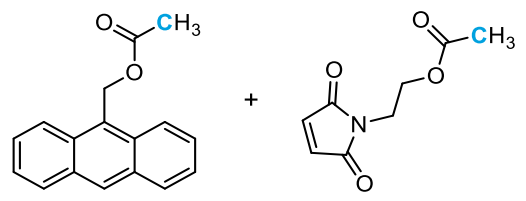

(i) Equilibrium Geometry

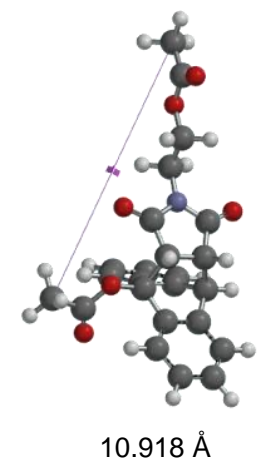

(ii) Immediately Prior to Bond Cleavage

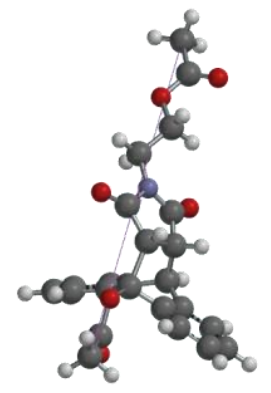

$14.668 \AA$

(iii) Immediately After Bond Cleavage

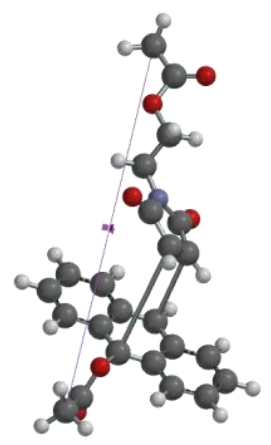

$14.718 \AA$ 

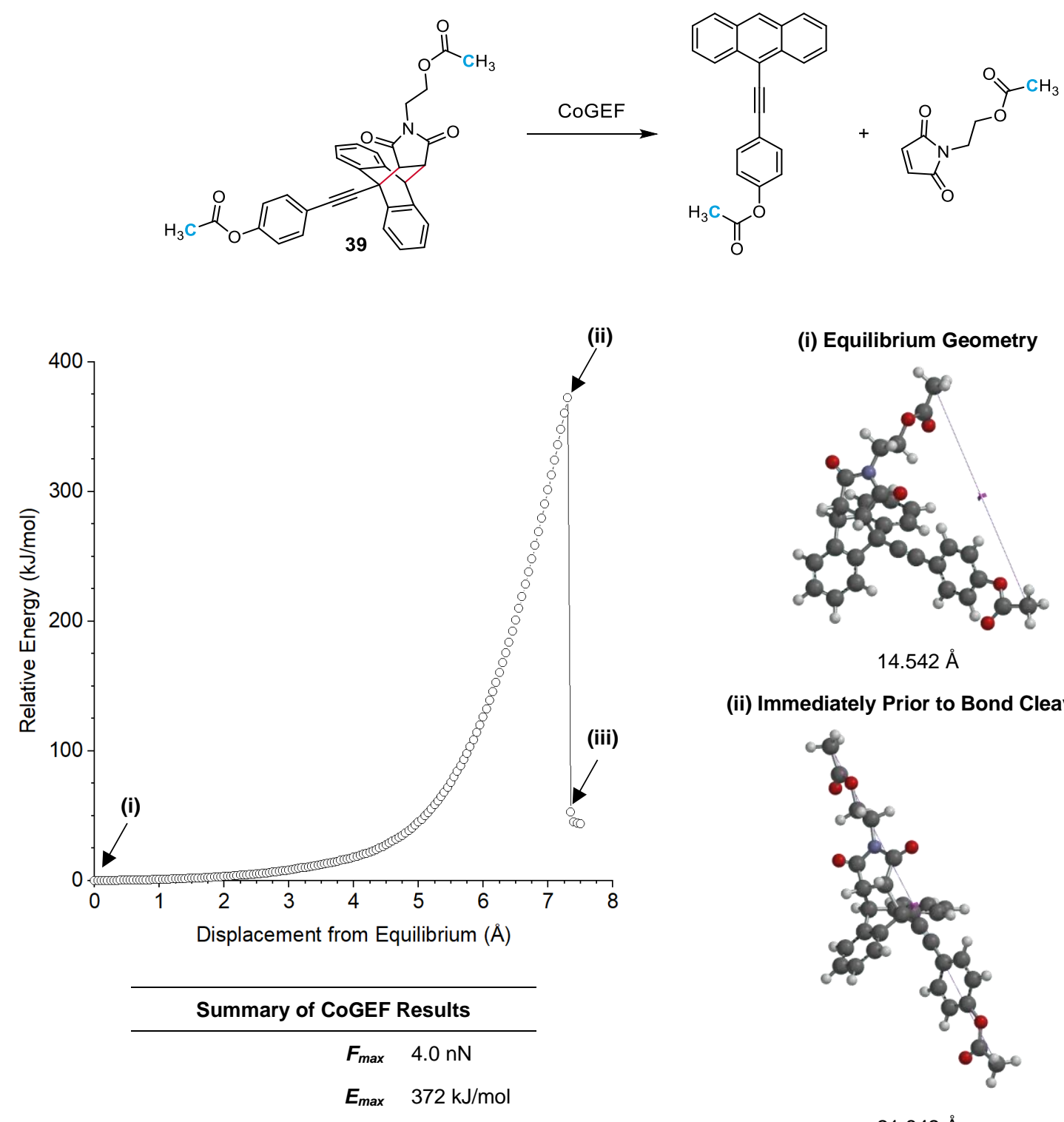

(ii) Immediately Prior to Bond Cleavage

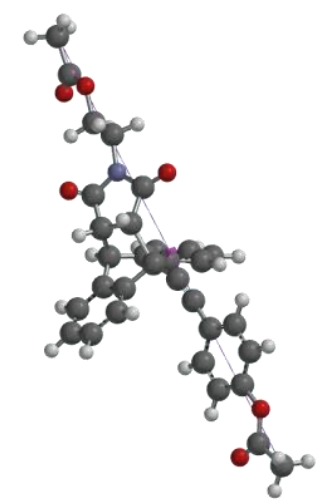

$21.842 \AA$

(iii) Immediately After Bond Cleavage

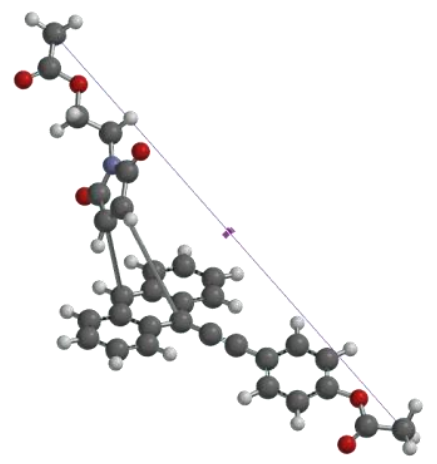

$21.892 \AA$ 


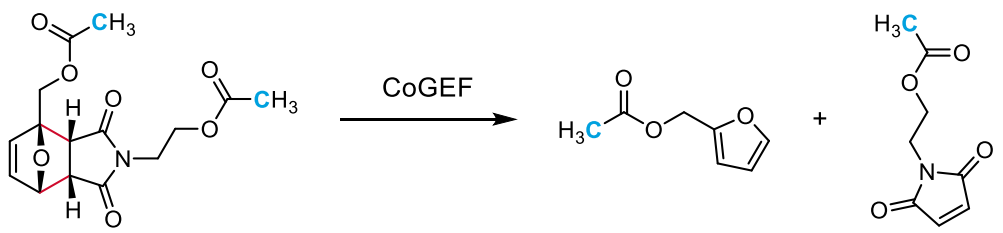

40

(ii)

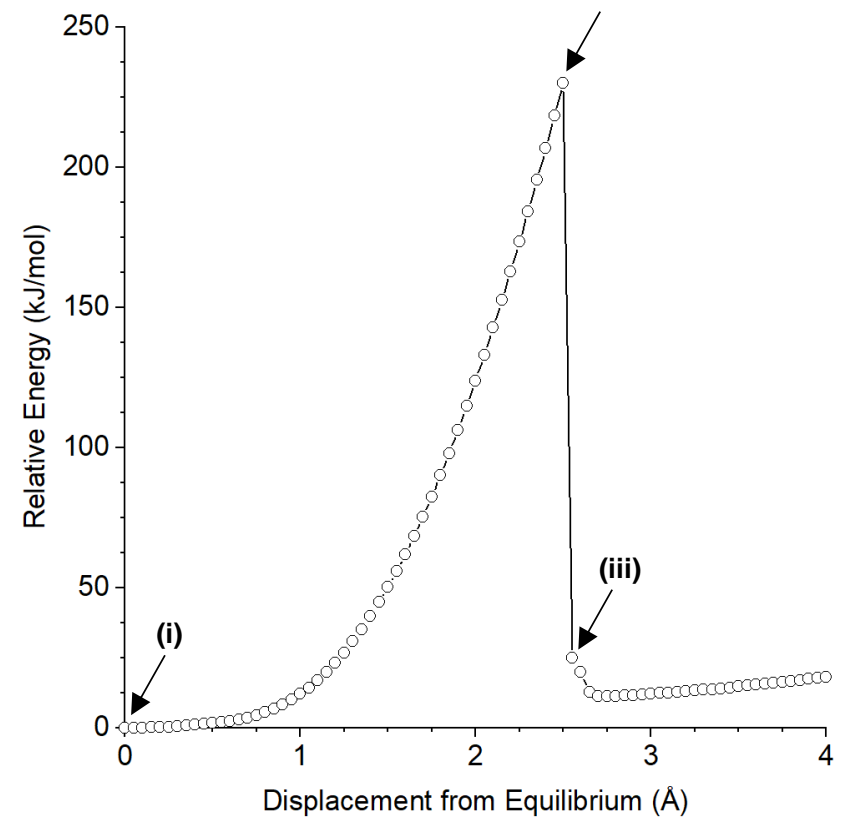

Summary of CoGEF Results

$\boldsymbol{F}_{\max } \quad 3.9 \mathrm{nN}$

$E_{\max } \quad 230 \mathrm{~kJ} / \mathrm{mol}$

Force-Bond Angle $26^{\circ}$ (i) Equilibrium Geometry

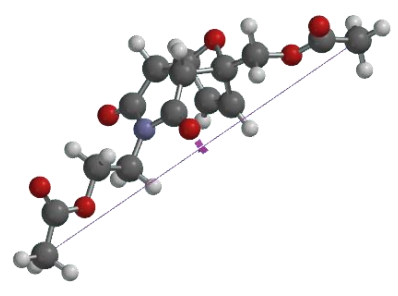

$13.422 \AA$

(ii) Immediately Prior to Bond Cleavage

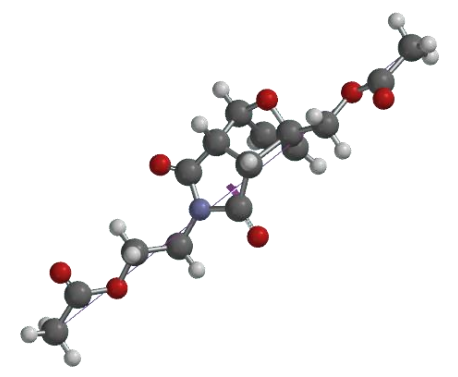

$15.922 \AA$

(iii) Immediately After Bond Cleavage

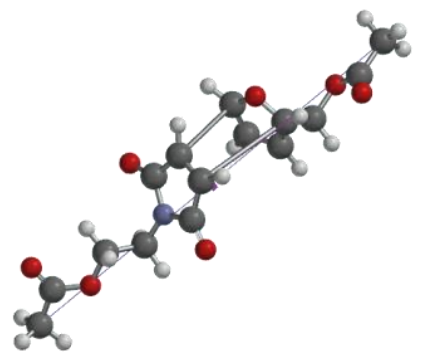

$15.972 \AA$ 

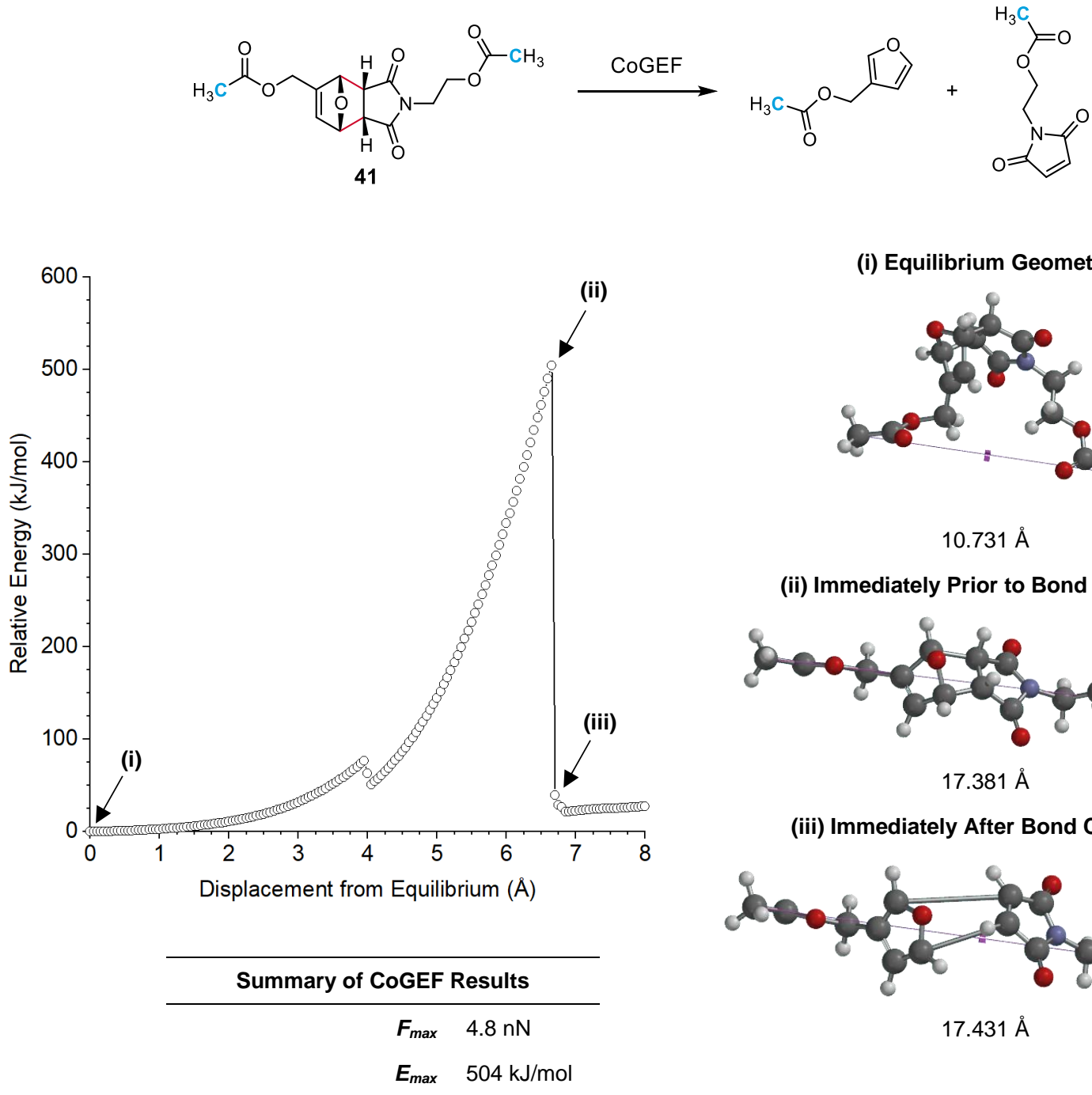

(i) Equilibrium Geometry

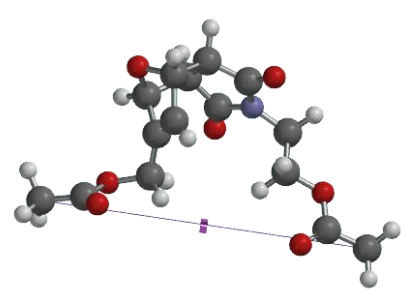

$10.731 \AA$

(ii) Immediately Prior to Bond Cleavage

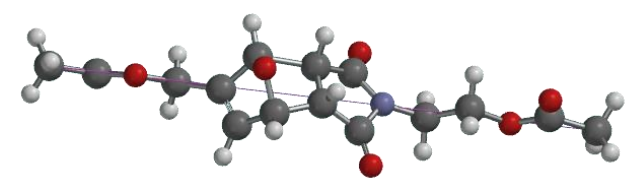

$17.381 \AA$

(iii) Immediately After Bond Cleavage

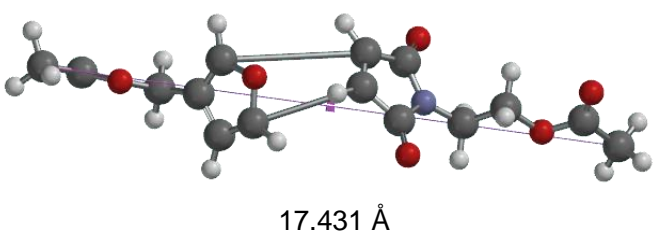

Force-Bond Angle $6.8^{\circ}$ 


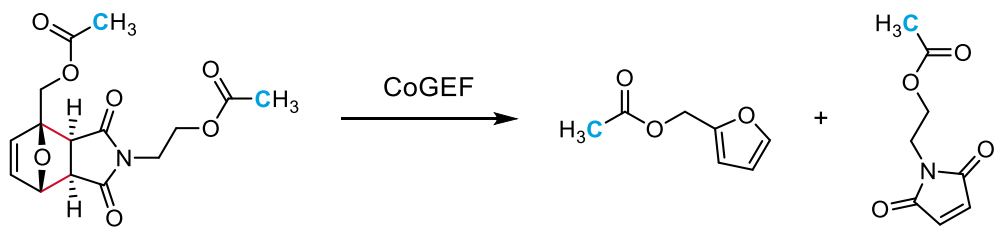

42

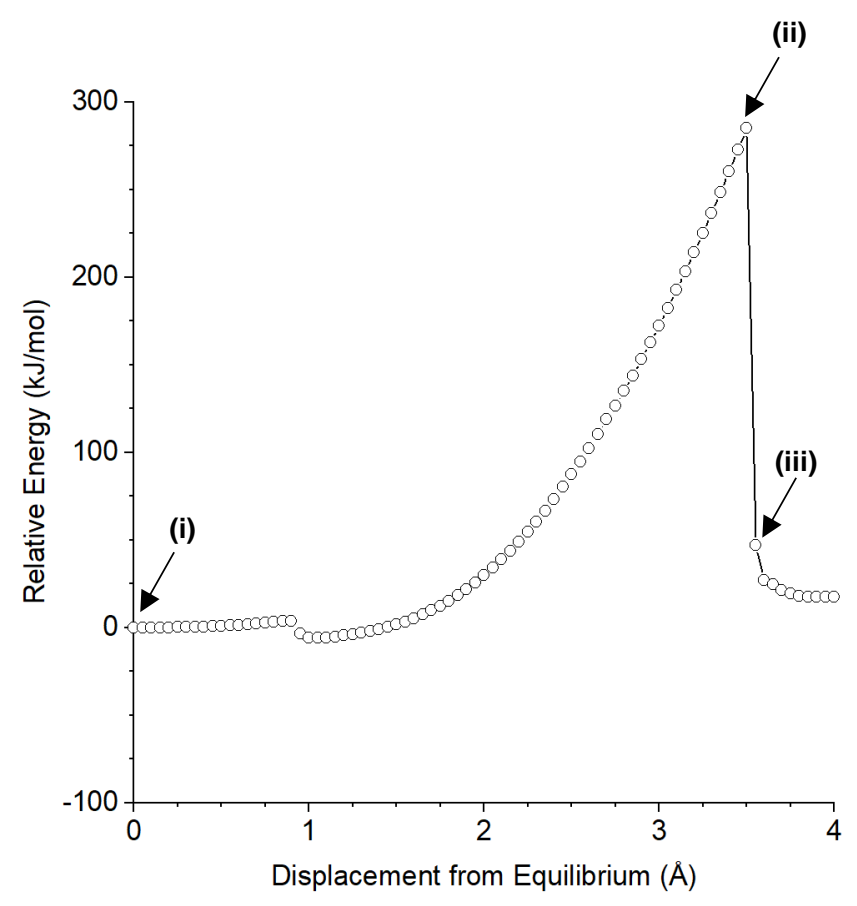

(i) Equilibrium Geometry

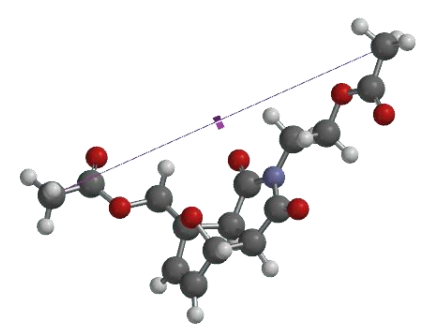

$12.433 \AA$

(ii) Immediately Prior to Bond Cleavage

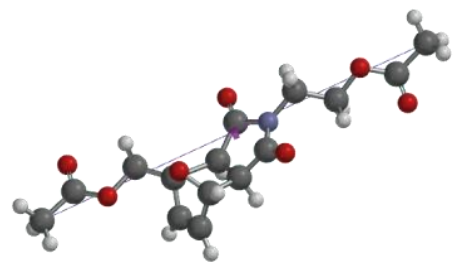

$15.933 \AA ̊$

(iii) Immediately After Bond Cleavage

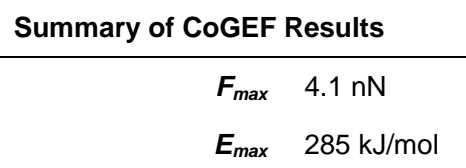

Force-Bond Angle $22^{\circ}$

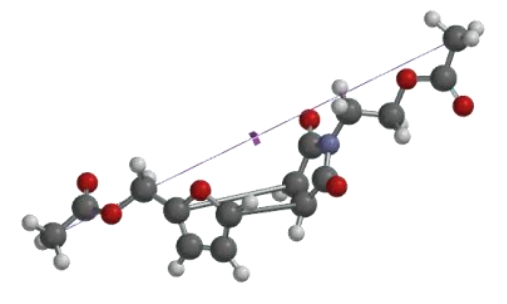

$15.983 \AA$ 


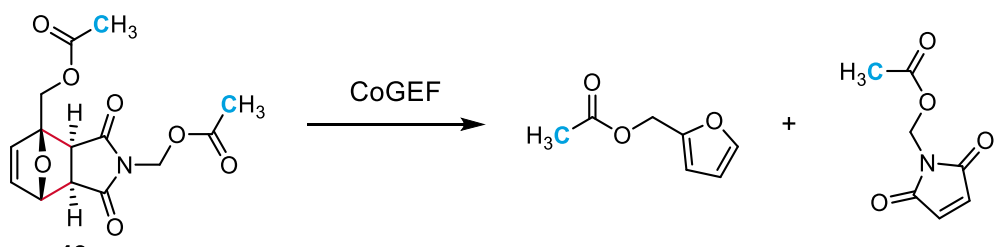

43

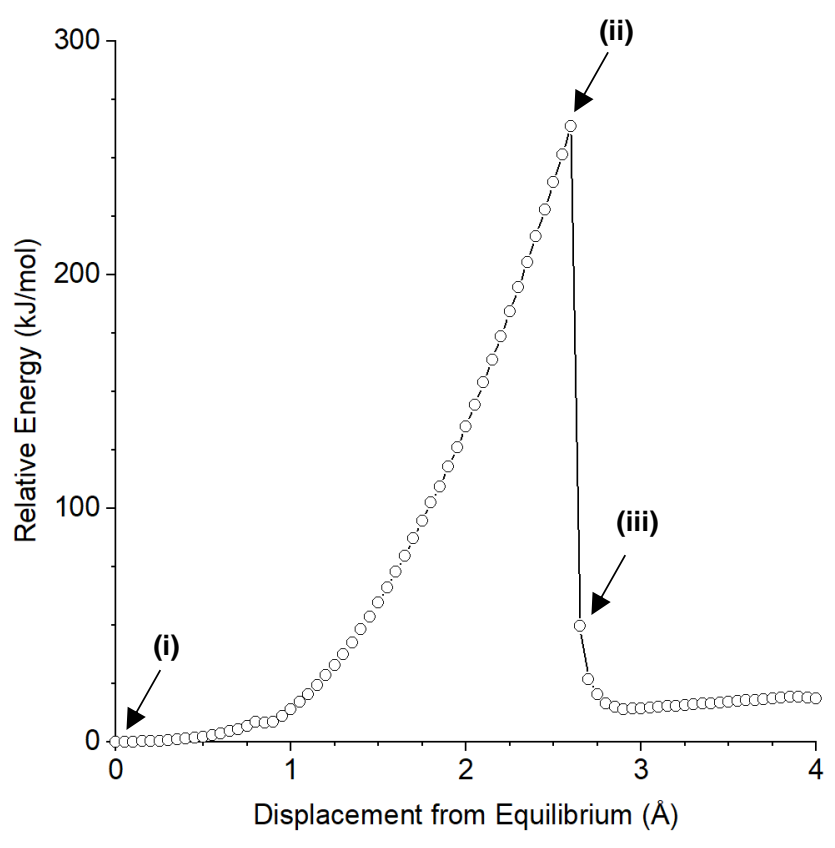

Summary of CoGEF Results

$F_{\max } \quad 4.0 \mathrm{nN}$

$E_{\max } 264 \mathrm{~kJ} / \mathrm{mol}$

Force-Bond Angle $21^{\circ}$ (i) Equilibrium Geometry

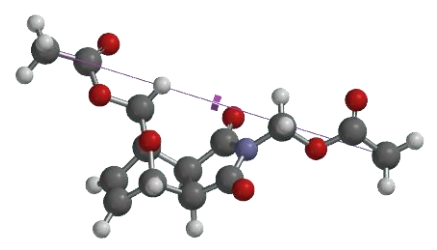

$11.874 \AA$

(ii) Immediately Prior to Bond Cleavage

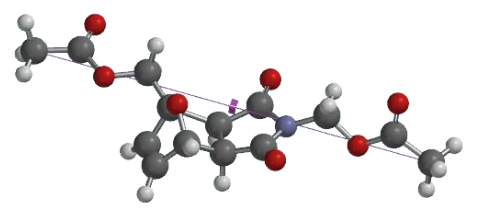

$14.474 \AA$

(iii) Immediately After Bond Cleavage

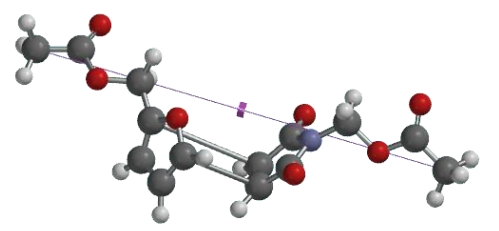

$14.524 \AA$ 

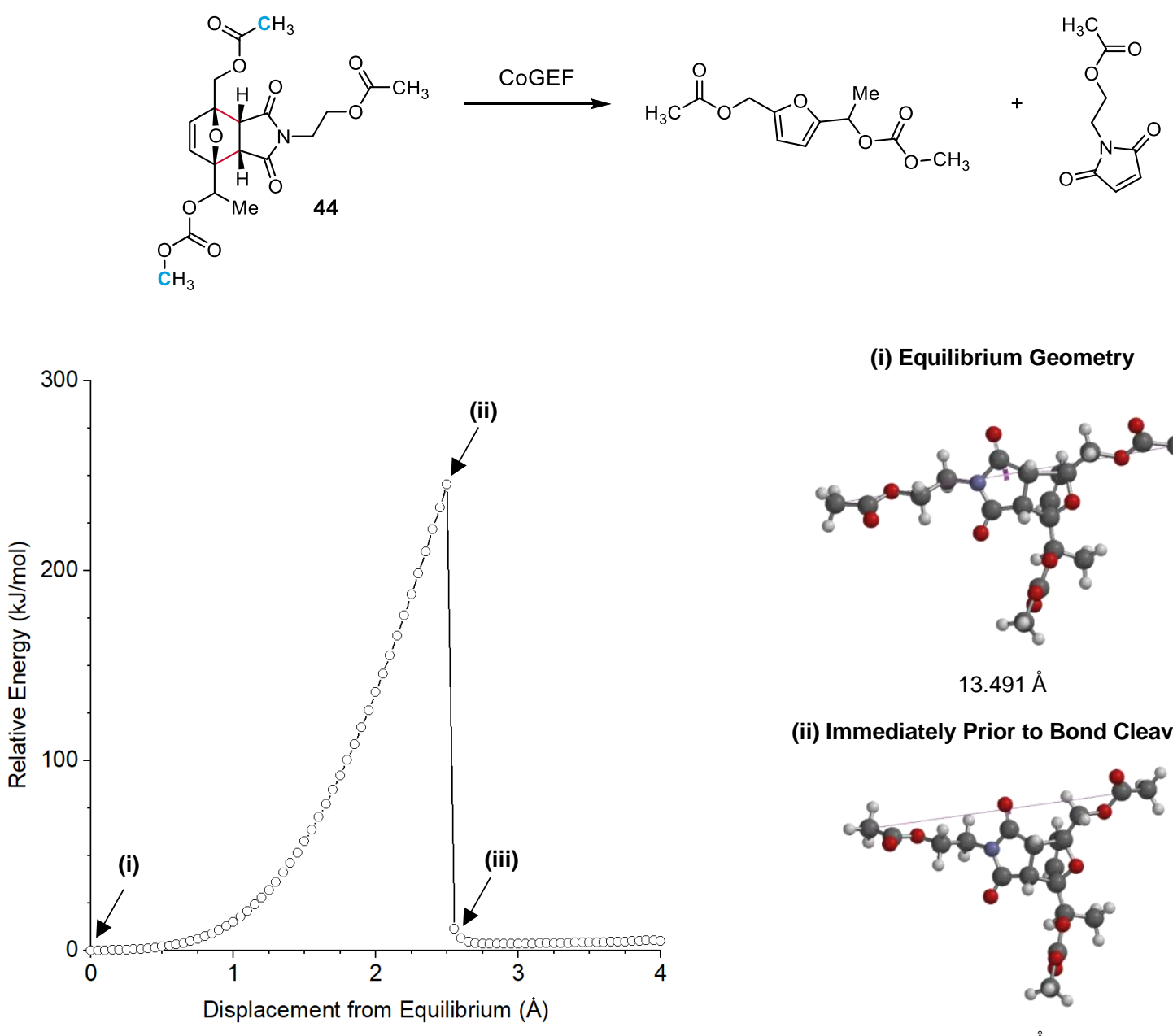

(i) Equilibrium Geometry

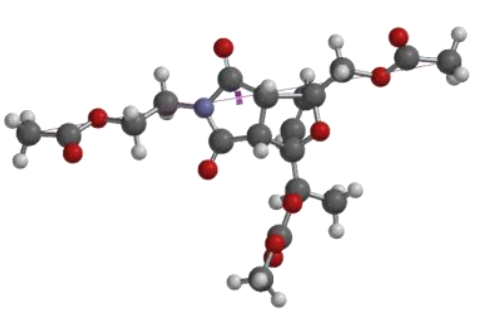

$13.491 \AA$

(ii) Immediately Prior to Bond Cleavage

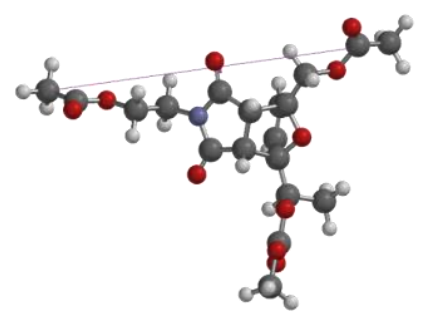

$15.991 \AA$

(iii) Immediately After Bond Cleavage

Summary of CoGEF Results

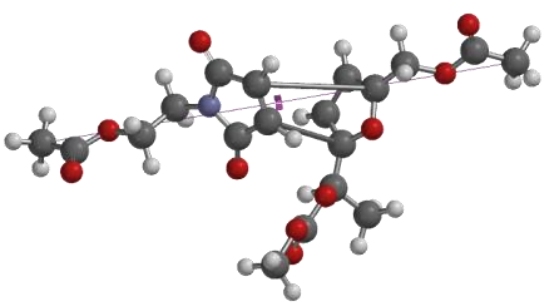

$16.041 \AA$ 


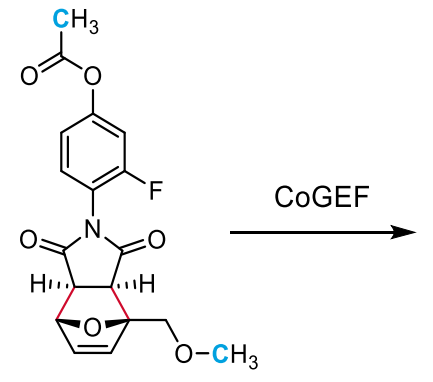

45

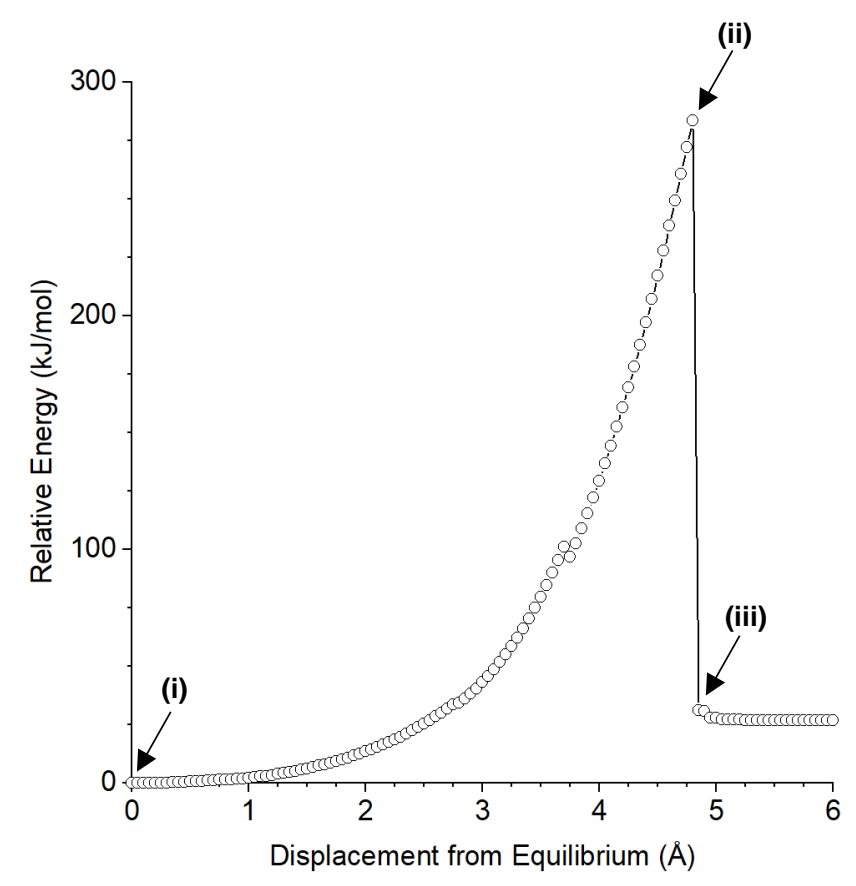

Summary of CoGEF Results

$$
\begin{array}{ll}
\boldsymbol{F}_{\max } & 3.8 \mathrm{nN} \\
\boldsymbol{E}_{\max } & 284 \mathrm{~kJ} / \mathrm{mol}
\end{array}
$$

Force-Bond Angle $21^{\circ}$

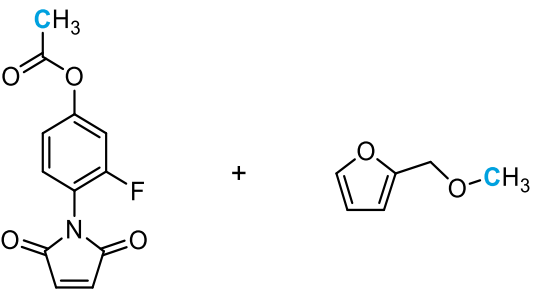

(i) Equilibrium Geometry

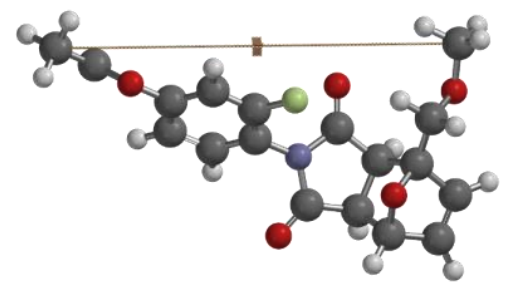

$11.583 \AA$

(ii) Immediately Prior to Bond Cleavage

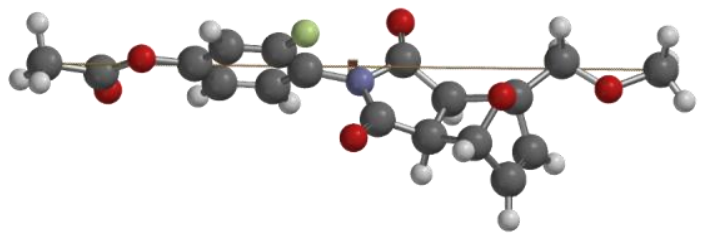

$16.383 \AA$

(iii) Immediately After Bond Cleavage

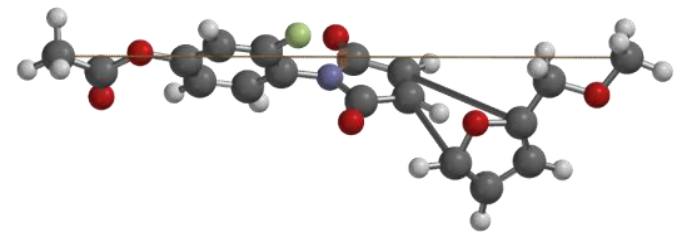

$16.433 \AA$ 


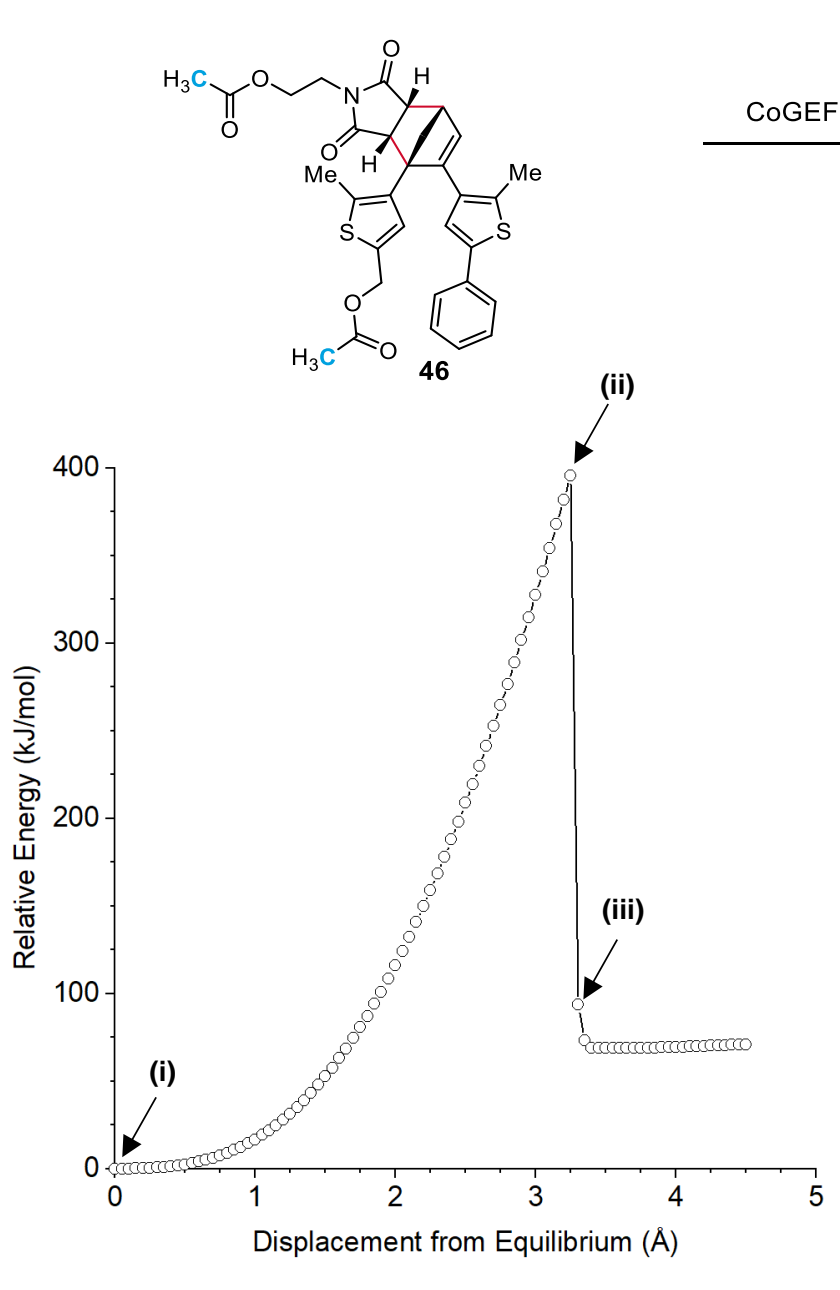

Summary of CoGEF Results

$\boldsymbol{F}_{\max } 4.6 \mathrm{nN}$

$E_{\max } \quad 396 \mathrm{~kJ} / \mathrm{mol}$

Force-Bond Angle $26^{\circ}$
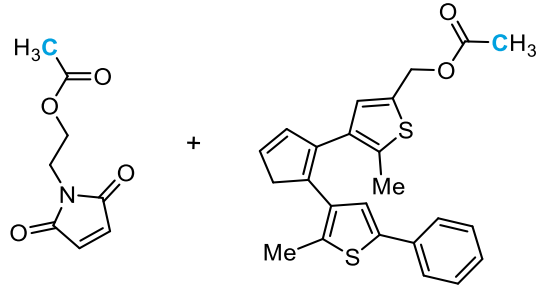

(i) Equilibrium Geometry

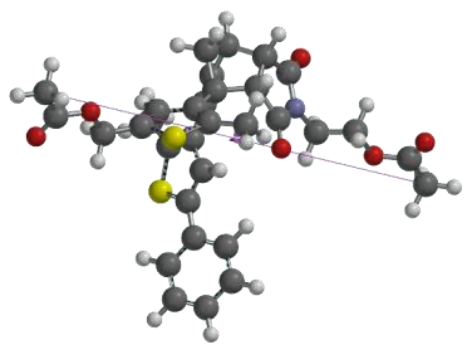

$17.075 \AA$

(ii) Immediately Prior to Bond Cleavage

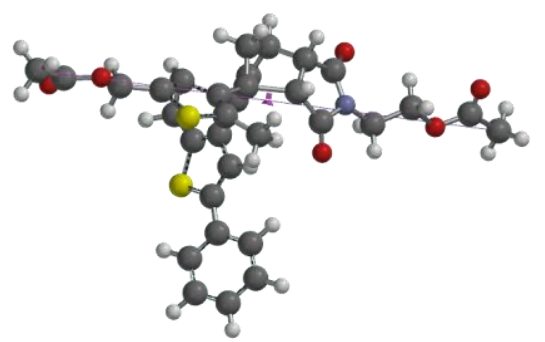

$20.325 \AA$

(iii) Immediately After Bond Cleavage

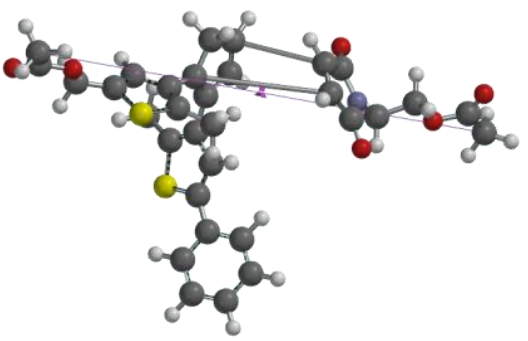

$20.375 \AA$ 


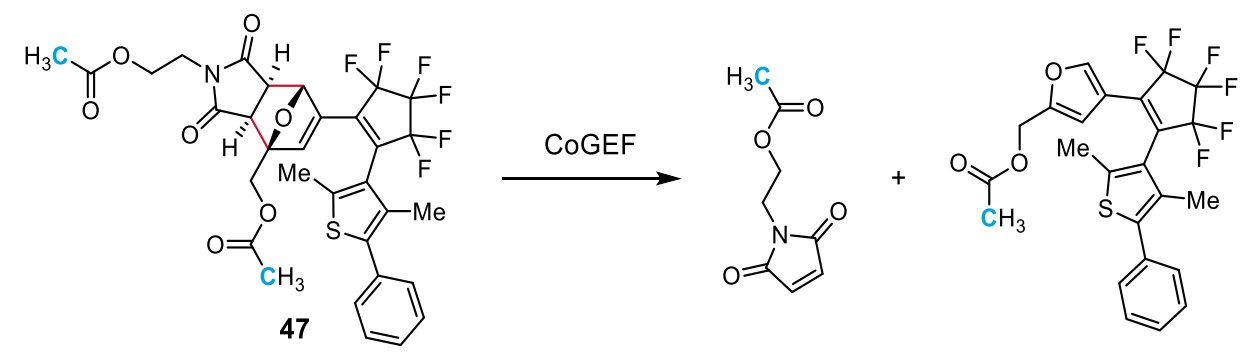

(i) Equilibrium Geometry
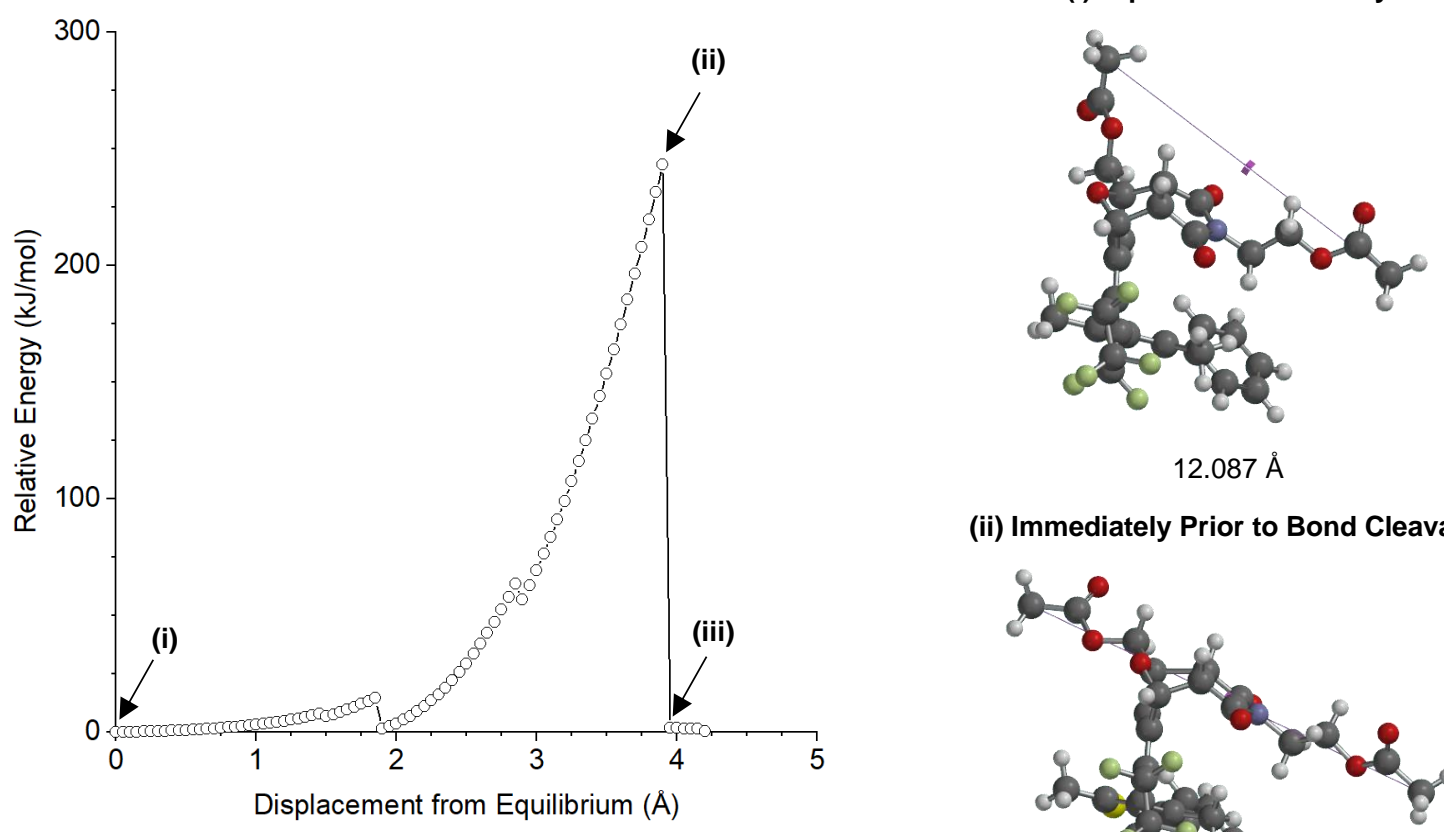

(ii) Immediately Prior to Bond Cleavage

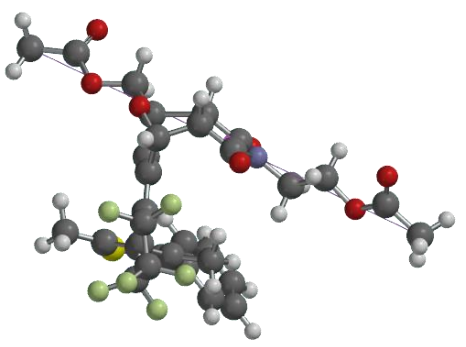

$15.987 \AA$

(iii) Immediately After Bond Cleavage

$\boldsymbol{F}_{\max } \quad 3.9 \mathrm{nN}$

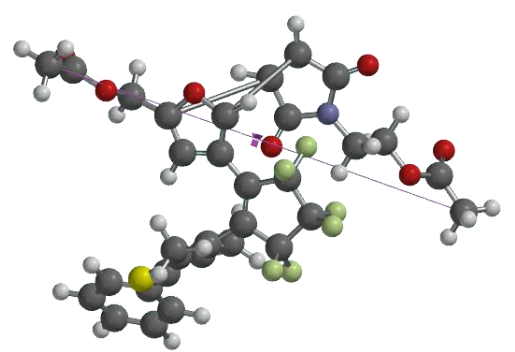

$16.037 \AA$ 

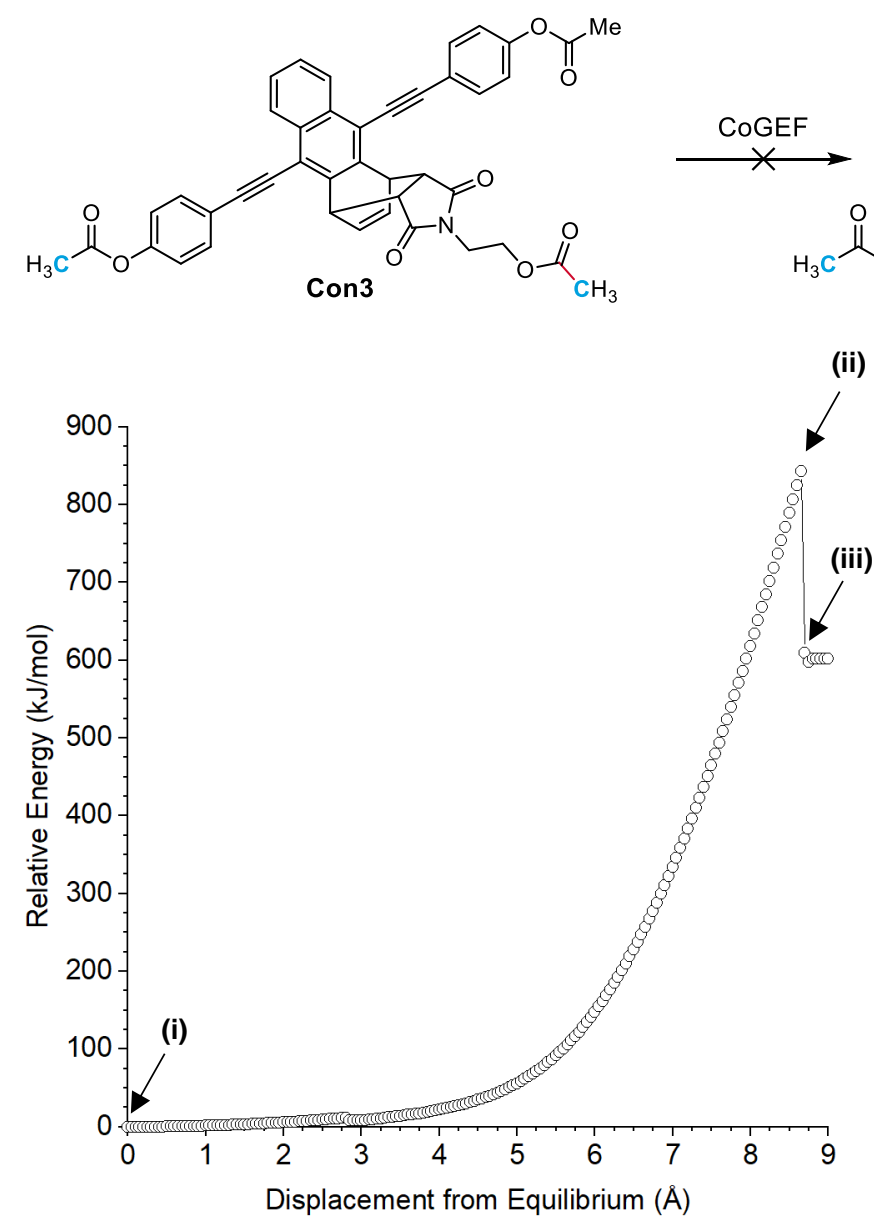

Summary of CoGEF Results

$$
\begin{array}{ll}
\boldsymbol{F}_{\max } & 6.0 \mathrm{nN} \\
\boldsymbol{E}_{\max } & 843 \mathrm{~kJ} / \mathrm{mol}
\end{array}
$$

Force-Bond Angle $29^{\circ}$

(ii)

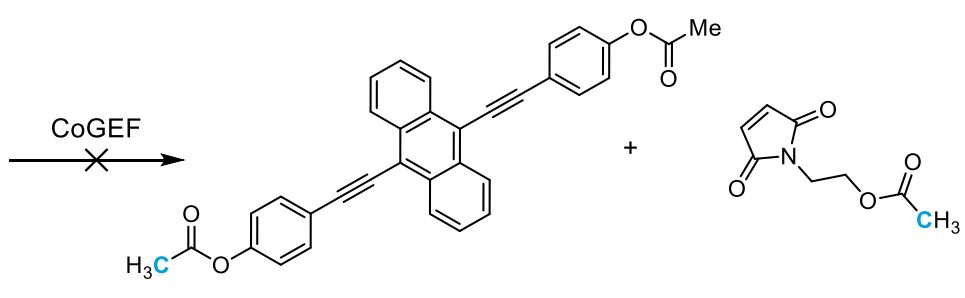

(i) Equilibrium Geometry

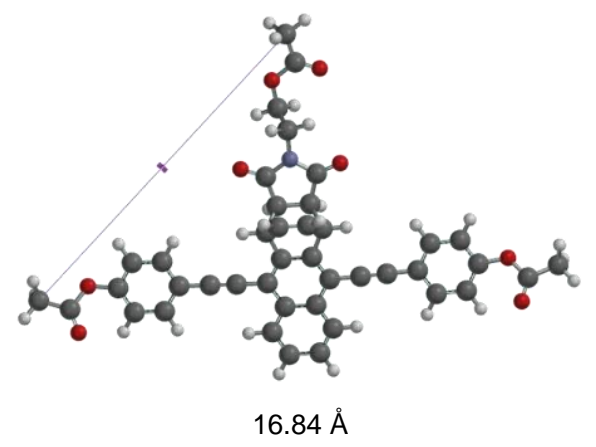

(ii) Immediately Prior to Bond Cleavage

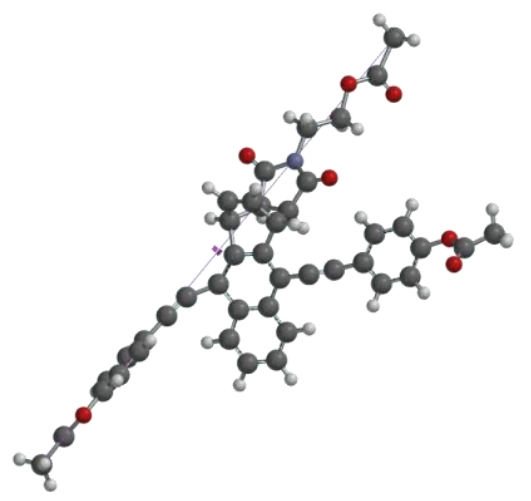

$25.49 \AA$

(iii) Immediately After Bond Cleavage

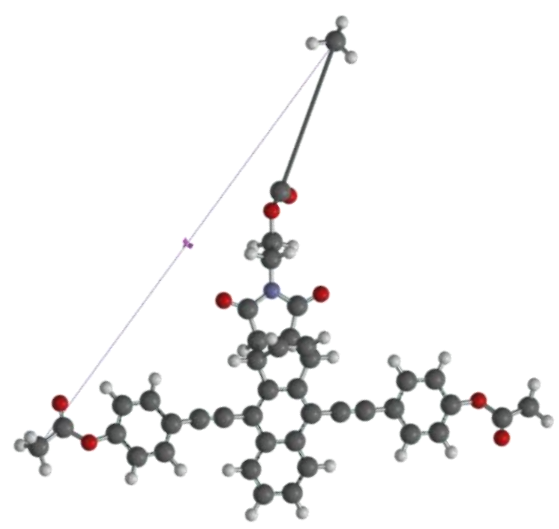

$25.54 \AA$ 

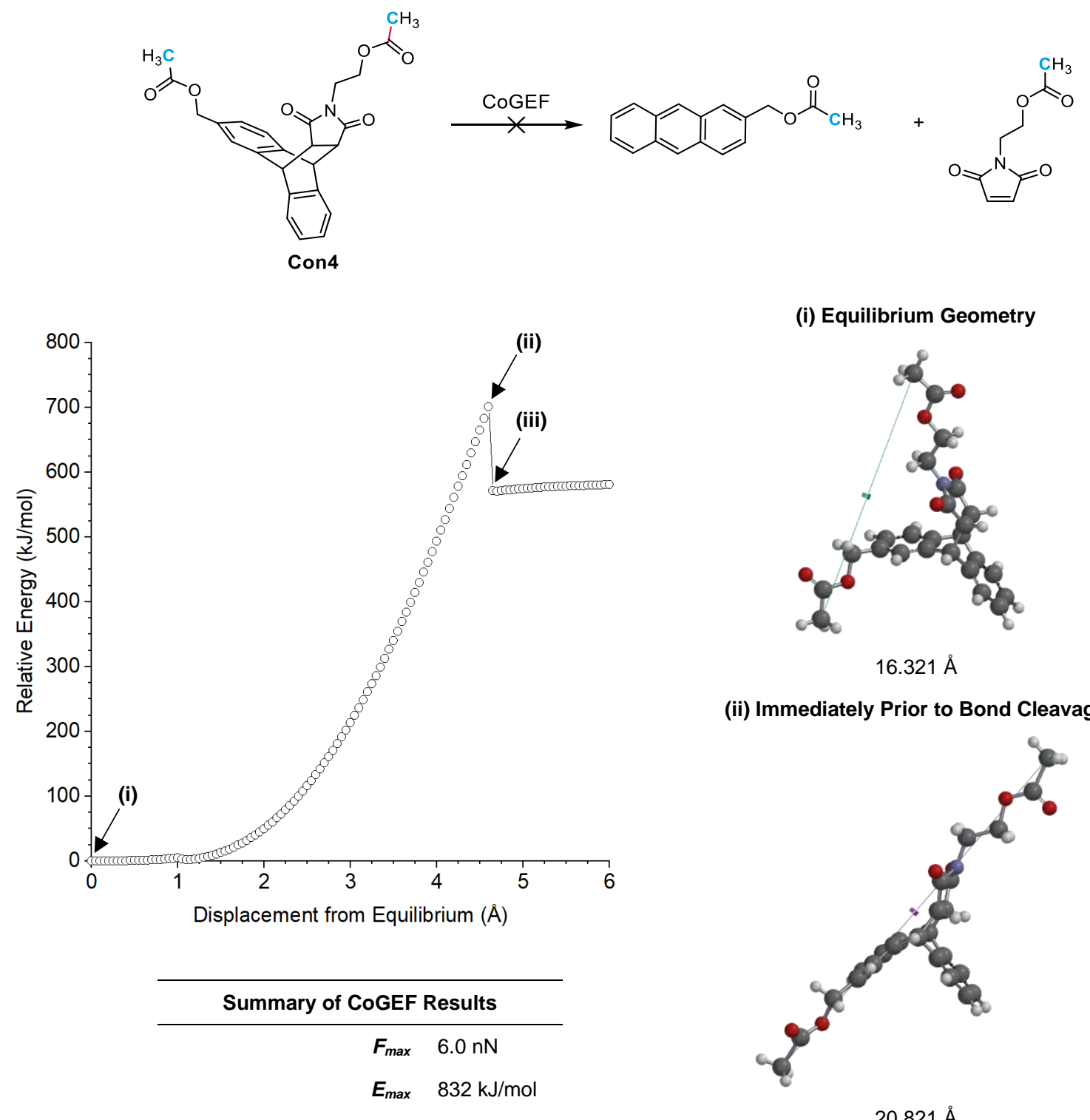

(i) Equilibrium Geometry

Force-Bond Angle $\quad 15^{\circ}$

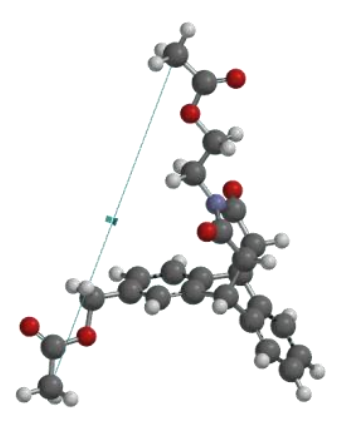

$16.321 \AA$

(ii) Immediately Prior to Bond Cleavage

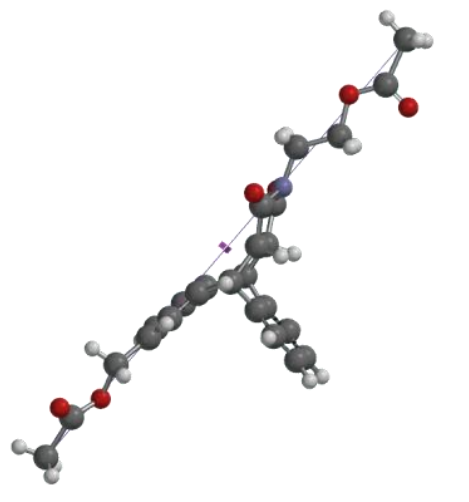

$20.821 \AA$

(iii) Immediately After Bond Cleavage

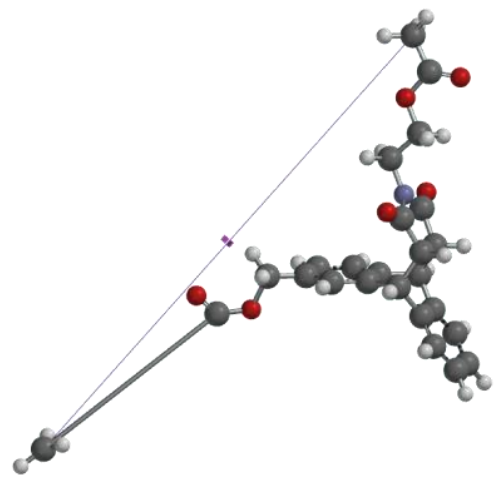

$20.871 \AA$ 
<smiles>CC(=O)OCCN1C(=O)[C@H]2C3C(=O)N1C[C@H]3C1C=C(COC(C)=O)[C@@H]2O1</smiles>

Con5

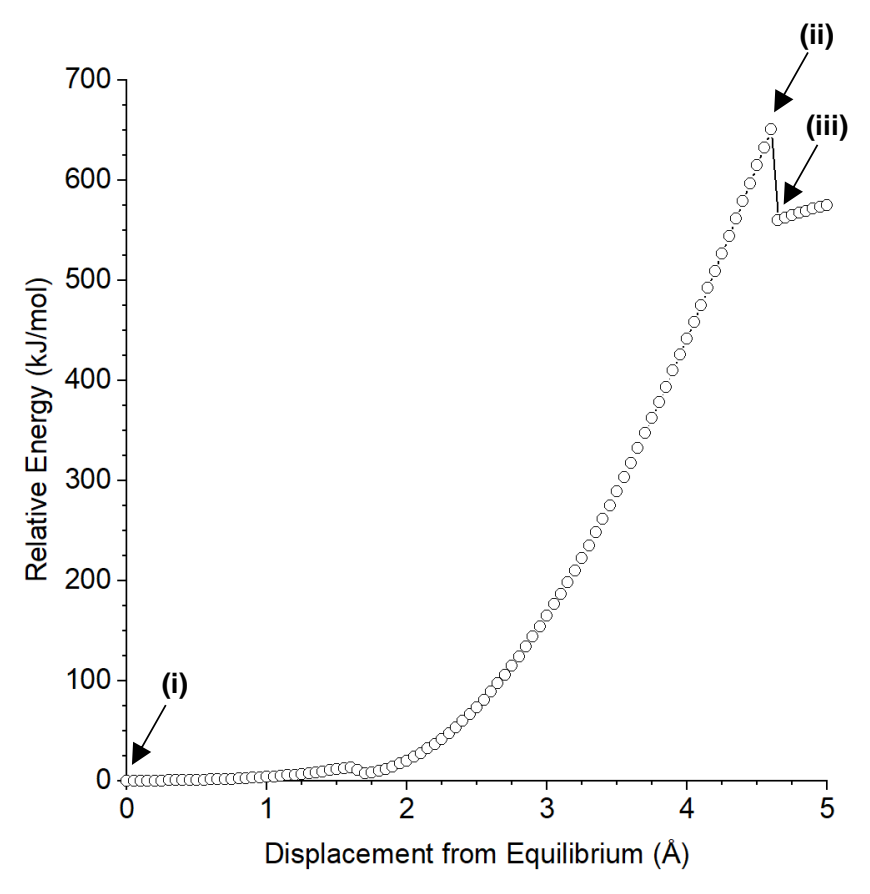

Summary of CoGEF Results

$$
\begin{array}{ll}
\boldsymbol{F}_{\max } & 6.0 \mathrm{nN} \\
\boldsymbol{E}_{\max } & 650 \mathrm{~kJ} / \mathrm{mol}
\end{array}
$$

Force-Bond Angle $39^{\circ}$<smiles>CC(=O)OCCN1C(=O)C=CC1=O</smiles>

(i) Equilibrium Geometry

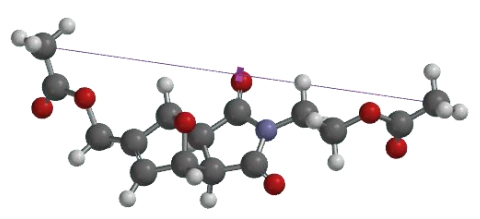

$13.761 \AA$

(ii) Immediately Prior to Bond Cleavage

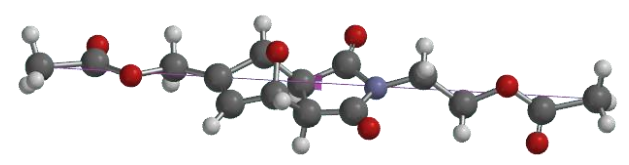

$18.361 \AA$

(iii) Immediately After Bond Cleavage

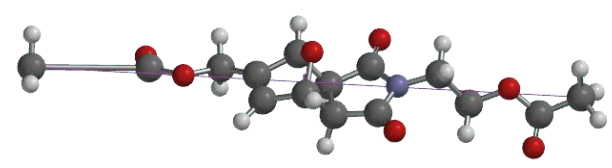

$18.411 \AA$ 

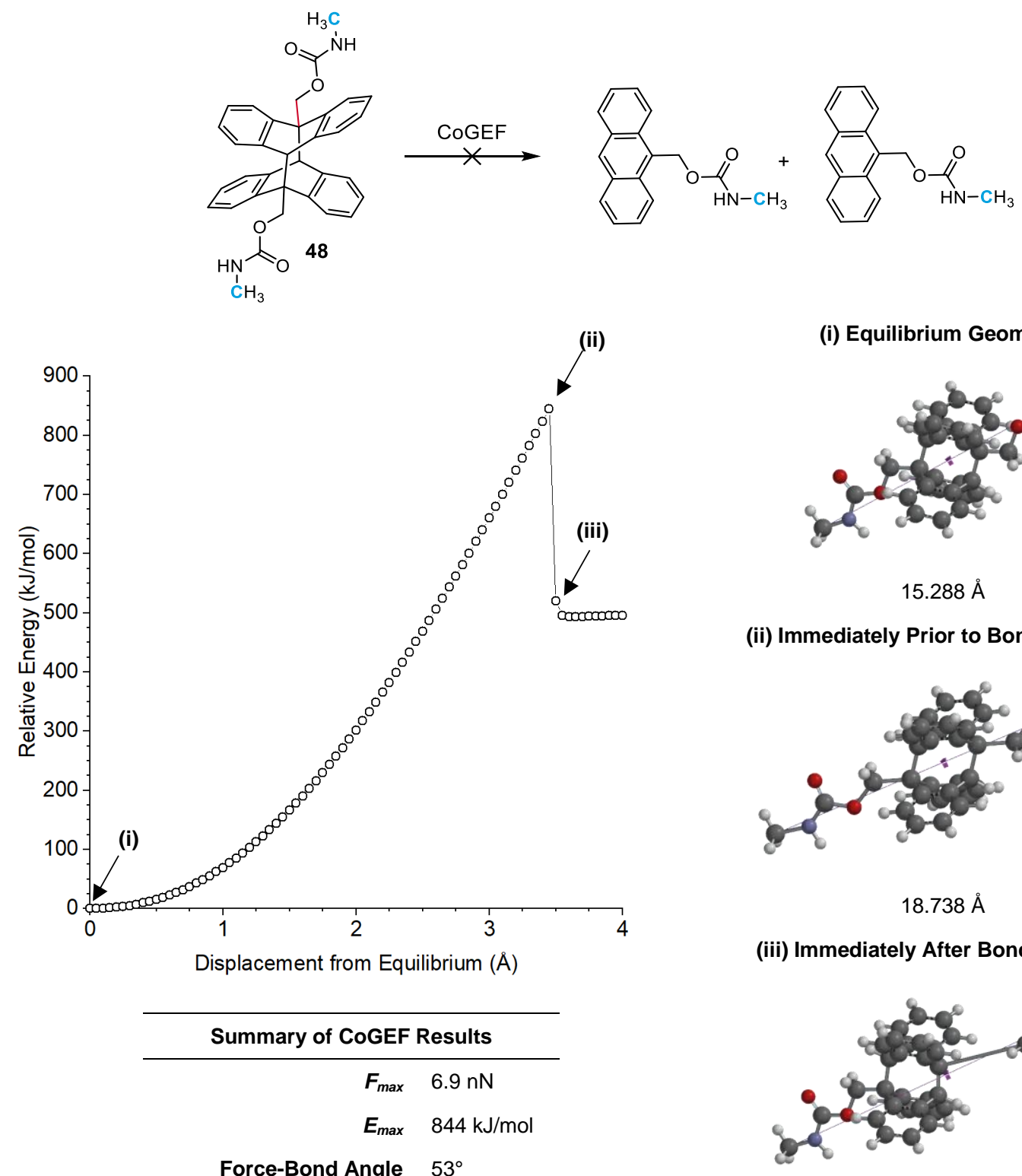

(i) Equilibrium Geometry

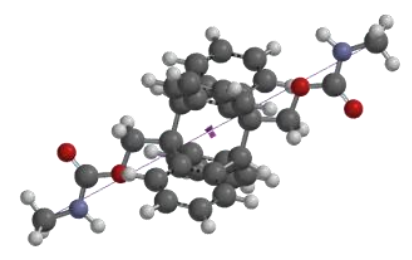

$15.288 \AA$

(ii) Immediately Prior to Bond Cleavage

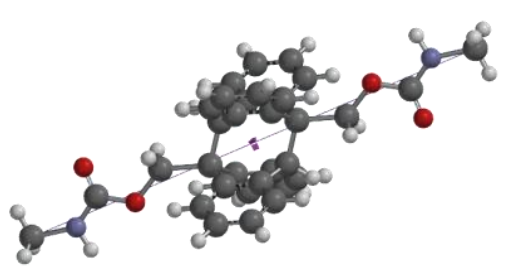

$18.738 \AA$

(iii) Immediately After Bond Cleavage

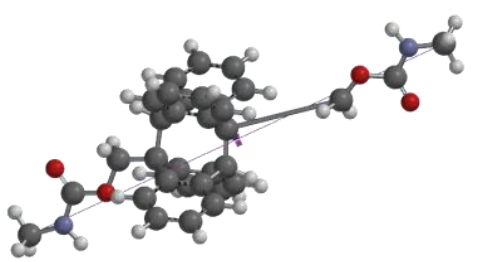

$18.788 \AA$ 

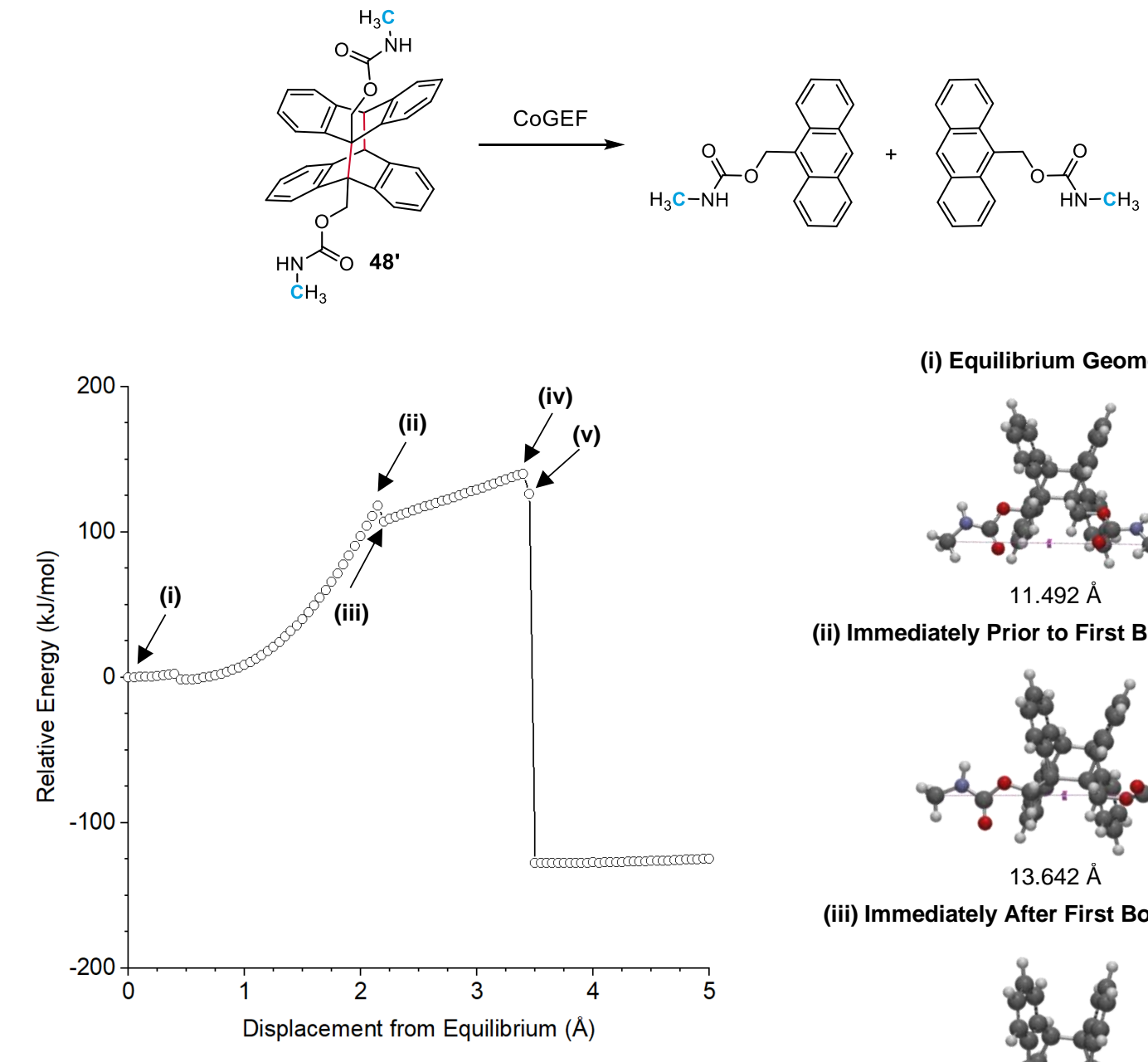

(i) Equilibrium Geometry

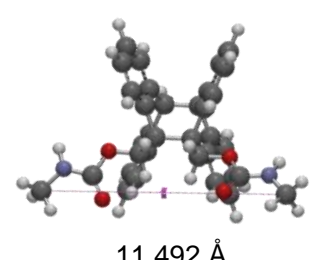

(ii) Immediately Prior to First Bond Cleavage

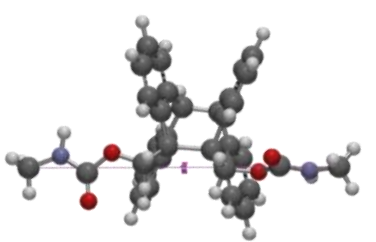

$13.642 \AA$

(iii) Immediately After First Bond Cleavage

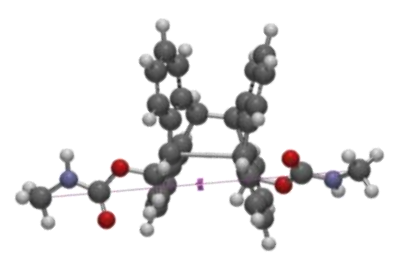

$13.692 \AA$

$\boldsymbol{F}_{\max } 2.3 \mathrm{nN}$

(iv) Immediately Prior to Second Bond Cleavage

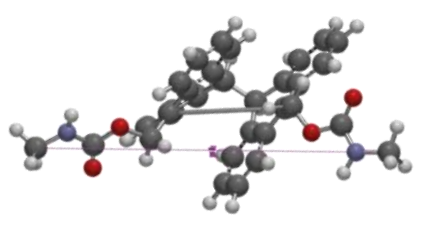

$14.892 \AA$

(v) Immediately After Second Bond Cleavage

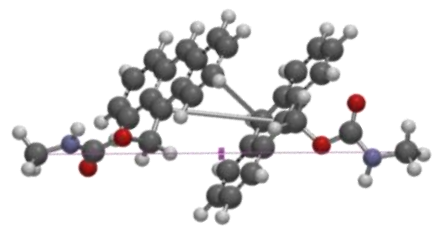

$14.942 \AA$ 

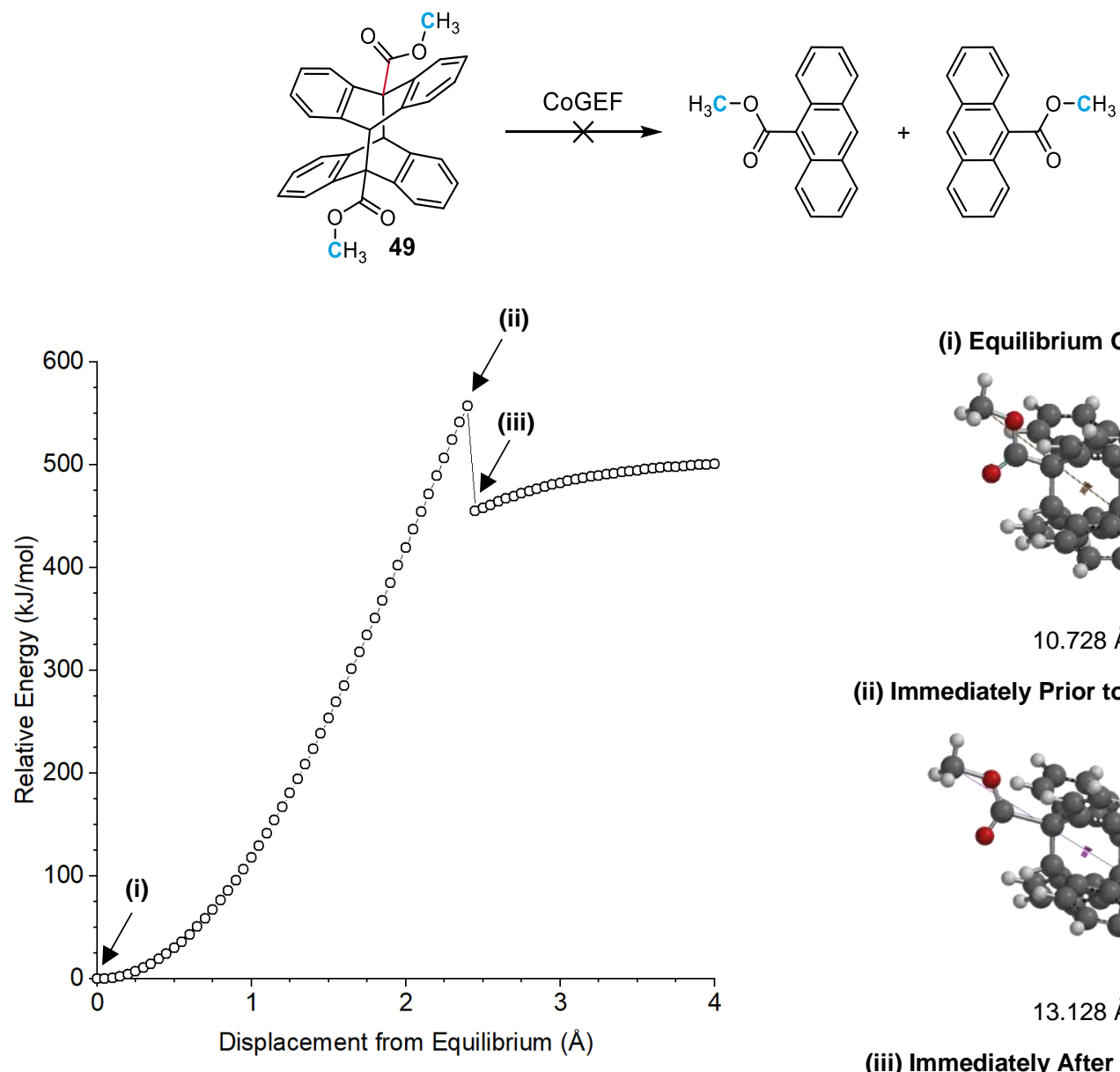

(i) Equilibrium Geometry

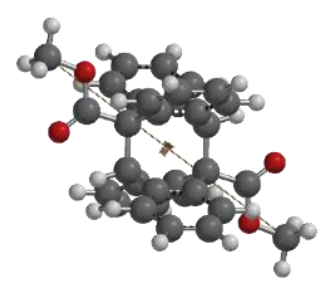

$10.728 \AA$

(ii) Immediately Prior to Bond Cleavage

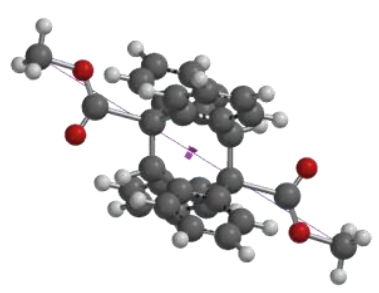

$13.128 \AA$

(iii) Immediately After Bond Cleavage

Summary of CoGEF Results

$$
\begin{array}{ll}
\boldsymbol{F}_{\max } & 5.8 \mathrm{nN} \\
\boldsymbol{E}_{\max } & 557 \mathrm{~kJ} / \mathrm{mol}
\end{array}
$$

Force-Bond Angle $57.8^{\circ}$

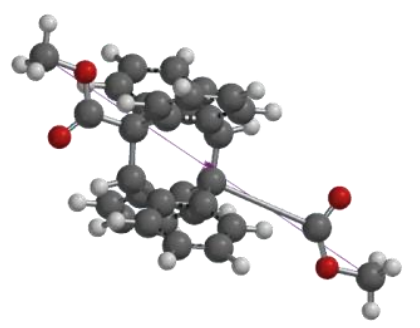

$13.178 \AA$ 

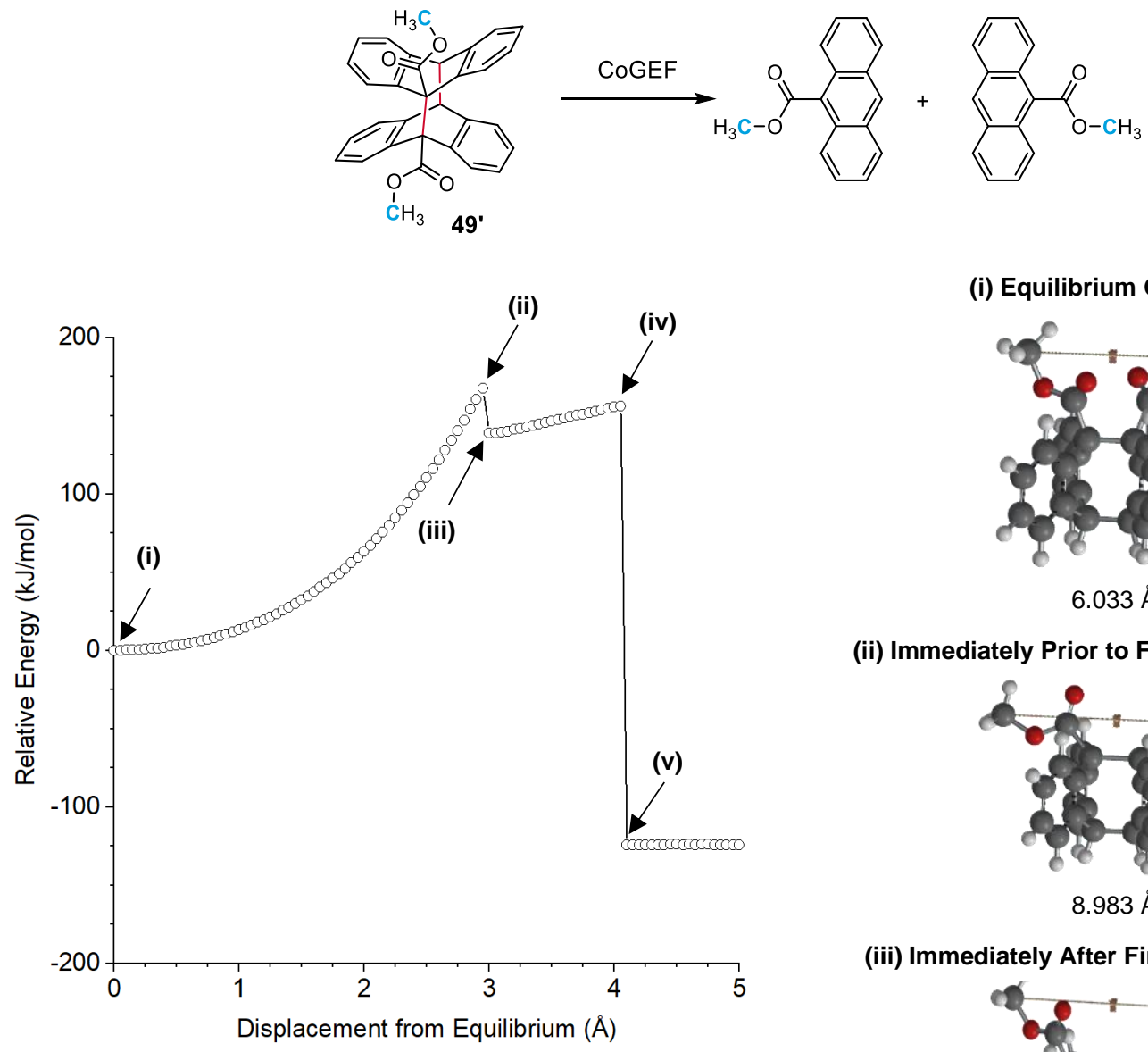

(i) Equilibrium Geometry

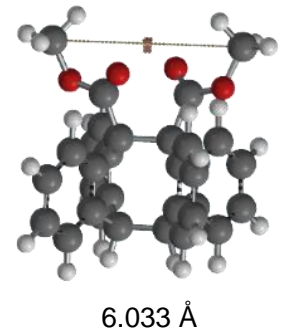

(ii) Immediately Prior to First Bond Cleavage

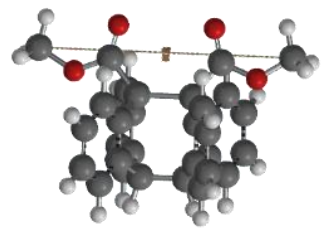

$8.983 \AA$

(iii) Immediately After First Bond Cleavage

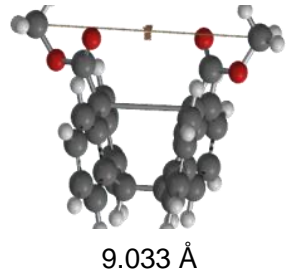

(iv) Immediately Prior to Second Bond Cleavage

Force-Bond Angle $0.5^{\circ}$

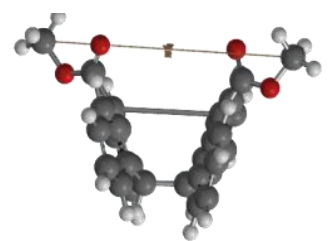

$10.083 \AA$

(v) Immediately After Second Bond Cleavage

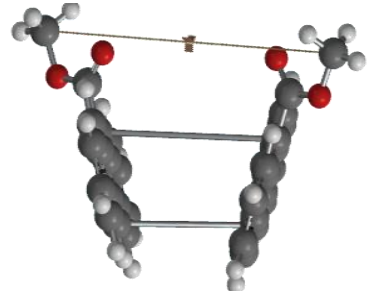

$10.133 \AA$ 
<smiles>CCCC(Cl)(Br)CCC</smiles>

(ii)

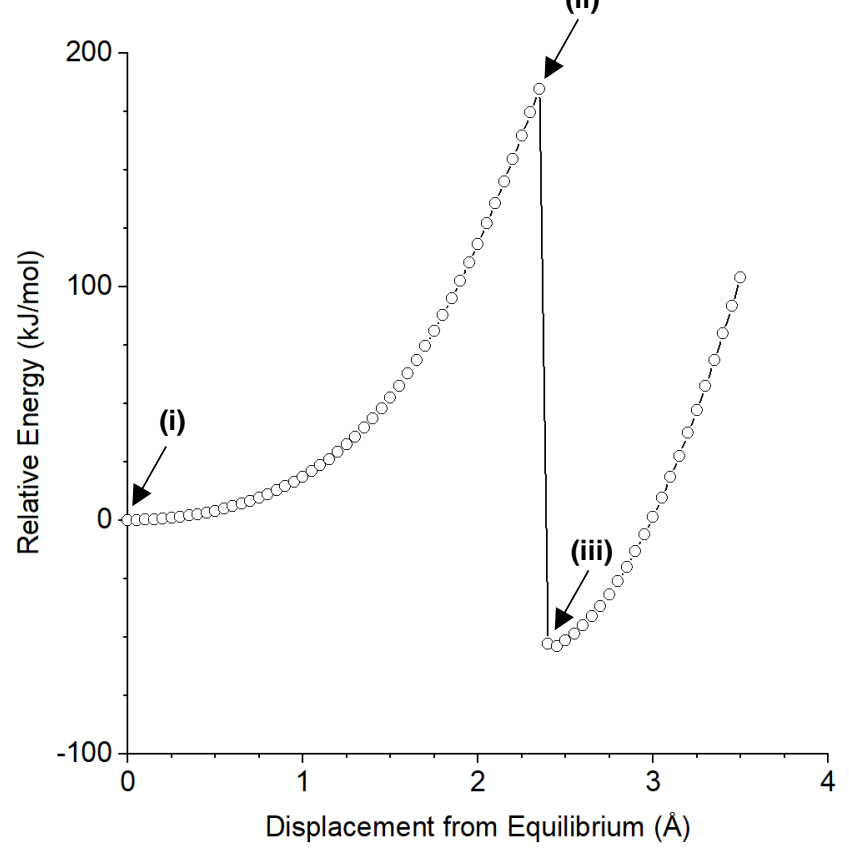

Summary of CoGEF Results

$F_{\text {max }} \quad 3.6 \mathrm{nN}$

$E_{\max } \quad 216 \mathrm{~kJ} / \mathrm{mol}$

Force-Bond Angle $\quad 0.1^{\circ}$<smiles>CCC/C=C(\Br)C(Cl)CCC</smiles>

(i) Equilibrium Geometry

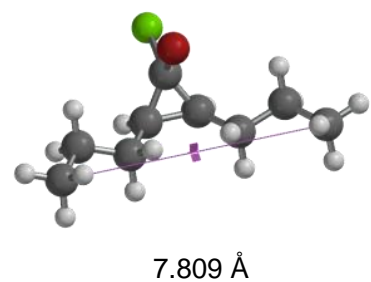

(ii) Immediately Prior to Bond Cleavage

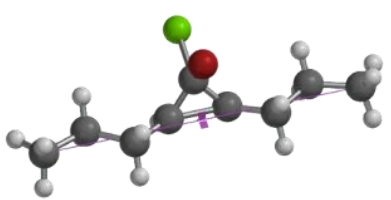

$10.359 \AA$

(iii) Immediately After Bond Cleavage

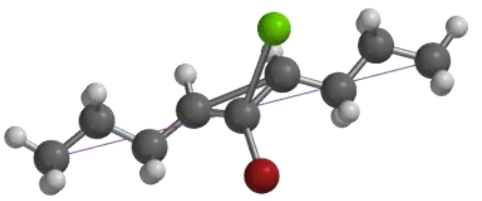

$10.359 \AA$ 


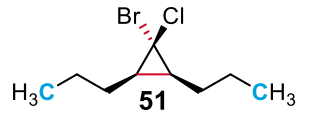

cate.

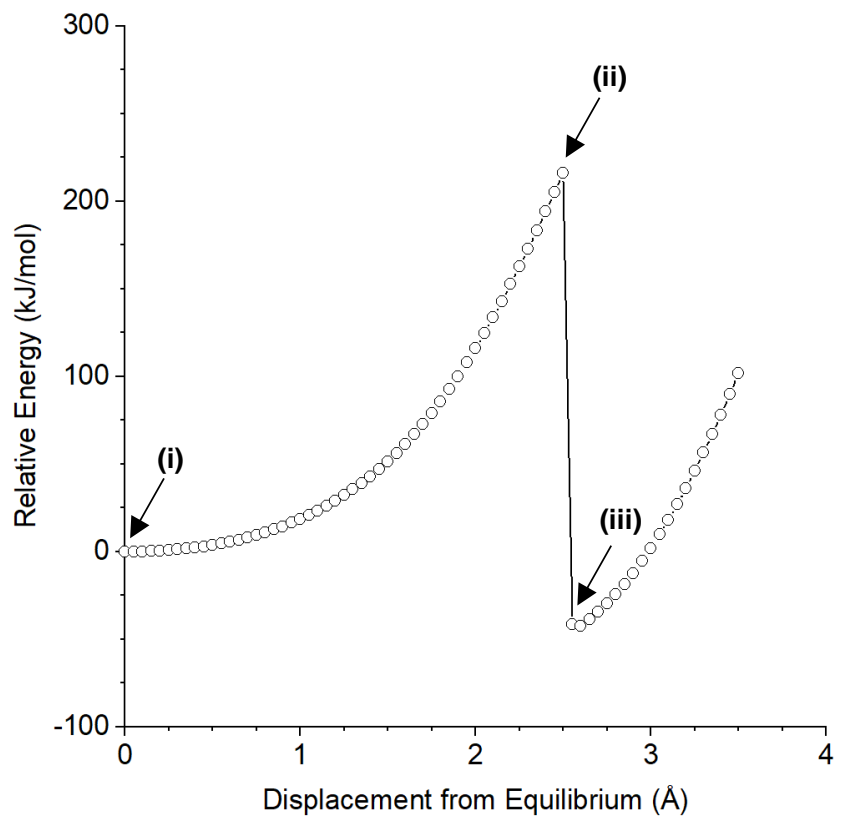

Summary of CoGEF Results

$F_{\max } \quad 3.3 \mathrm{nN}$

$E_{\max } 184 \mathrm{~kJ} / \mathrm{mol}$

Force-Bond Angle $\quad 0.1^{\circ}$ (i) Equilibrium Geometry

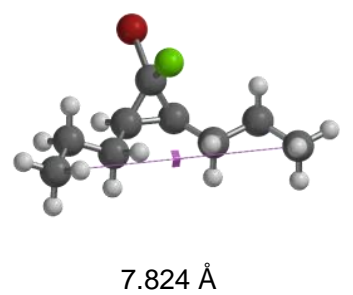

(ii) Immediately Prior to Bond Cleavage

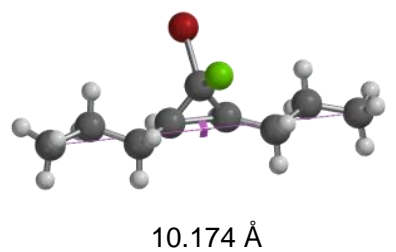

(iii) Immediately After Bond Cleavage

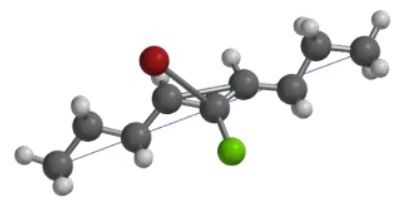

$10.224 \AA$ 
<smiles>CCCC1C(CCC)C1(F)Cl</smiles>

(ii)

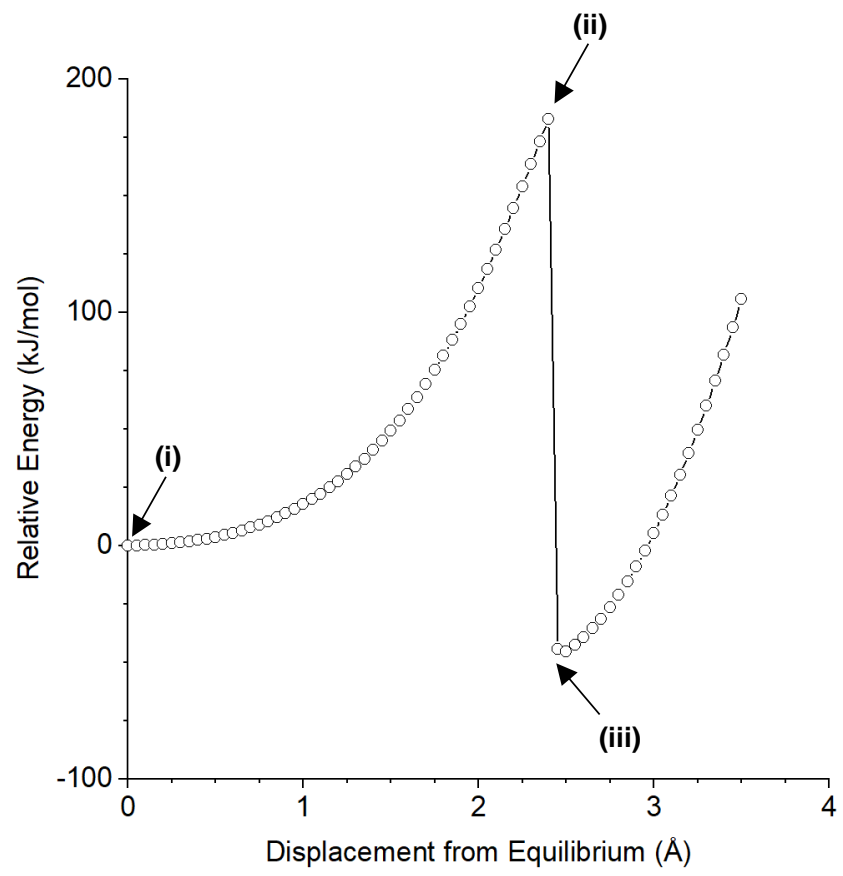

Summary of CoGEF Results

$\boldsymbol{F}_{\max } \quad 3.5 \mathrm{nN}$

$E_{\text {max }} 262 \mathrm{~kJ} / \mathrm{mol}$

Force-Bond Angle $\quad 0.0^{\circ}$ (i) Equilibrium Geometry

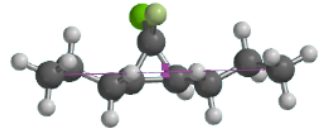

$7.776 \AA$

(ii) Immediately Prior to Bond Cleavage

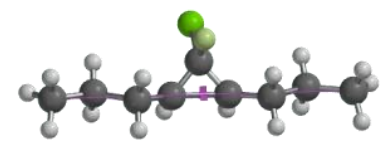

$10.176 \AA$

(iii) Immediately After Bond Cleavage

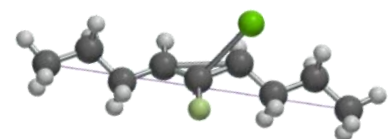

$10.226 \AA$ 
<smiles>CCCC(F)(Cl)CCC</smiles>

(ii)

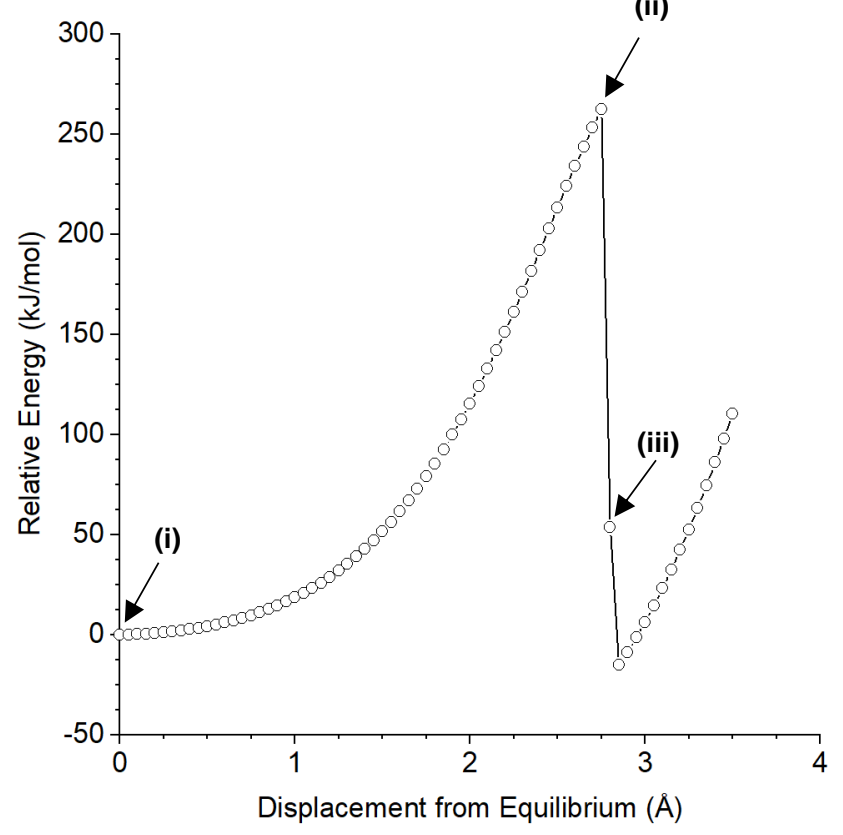

(i) Equilibrium Geometry

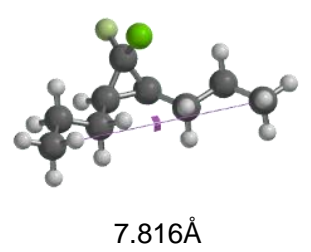

(ii) Immediately Prior to Bond Cleavage

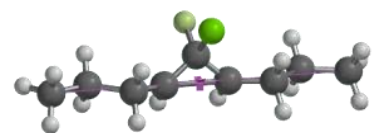

$10.566 \AA$

(iii) Immediately After Bond Cleavage

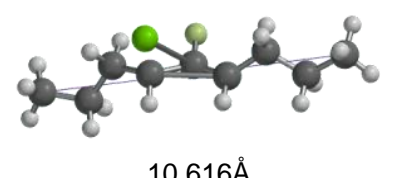

Summary of CoGEF Results

$$
\begin{array}{ll}
\boldsymbol{F}_{\max } & 3.2 \mathrm{nN} \\
\boldsymbol{E}_{\max } & 183 \mathrm{~kJ} / \mathrm{mol}
\end{array}
$$

Force-Bond Angle $\quad 0.1^{\circ}$ 

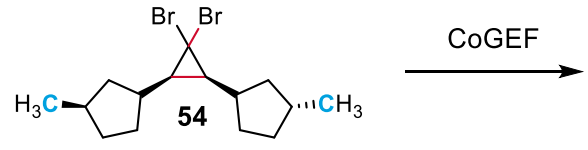

(ii)

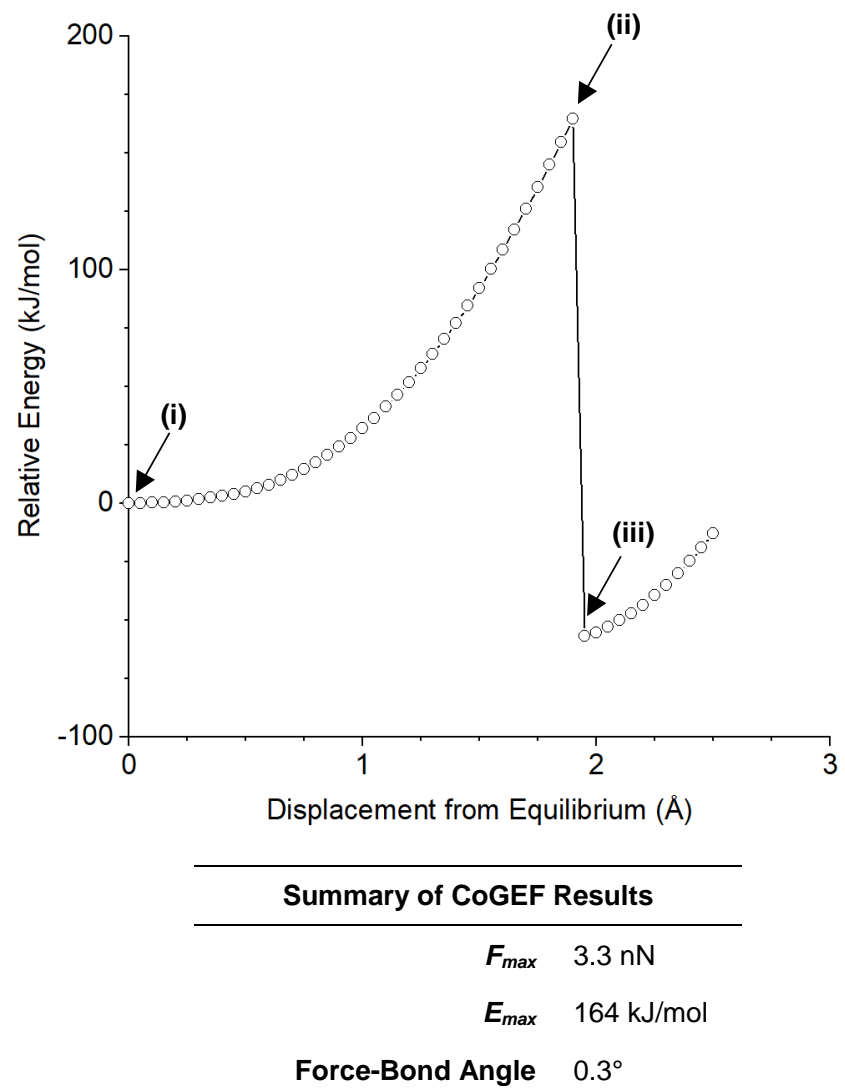

(i) Equilibrium Geometry

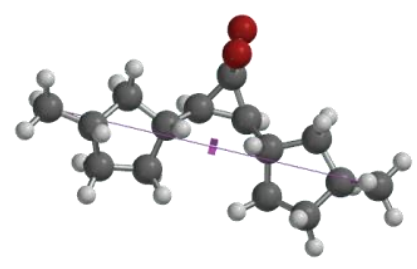

$10.675 \AA$

(ii) Immediately Prior to Bond Cleavage

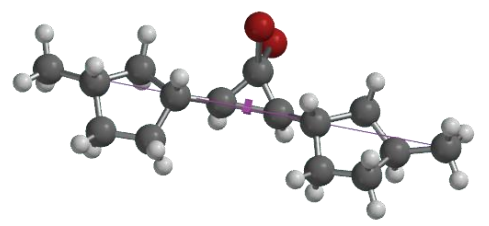

$12.575 \AA$

(iii) Immediately After Bond Cleavage

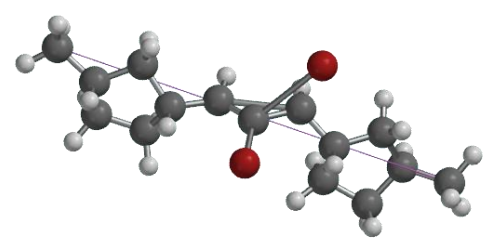

$12.625 \AA$ 

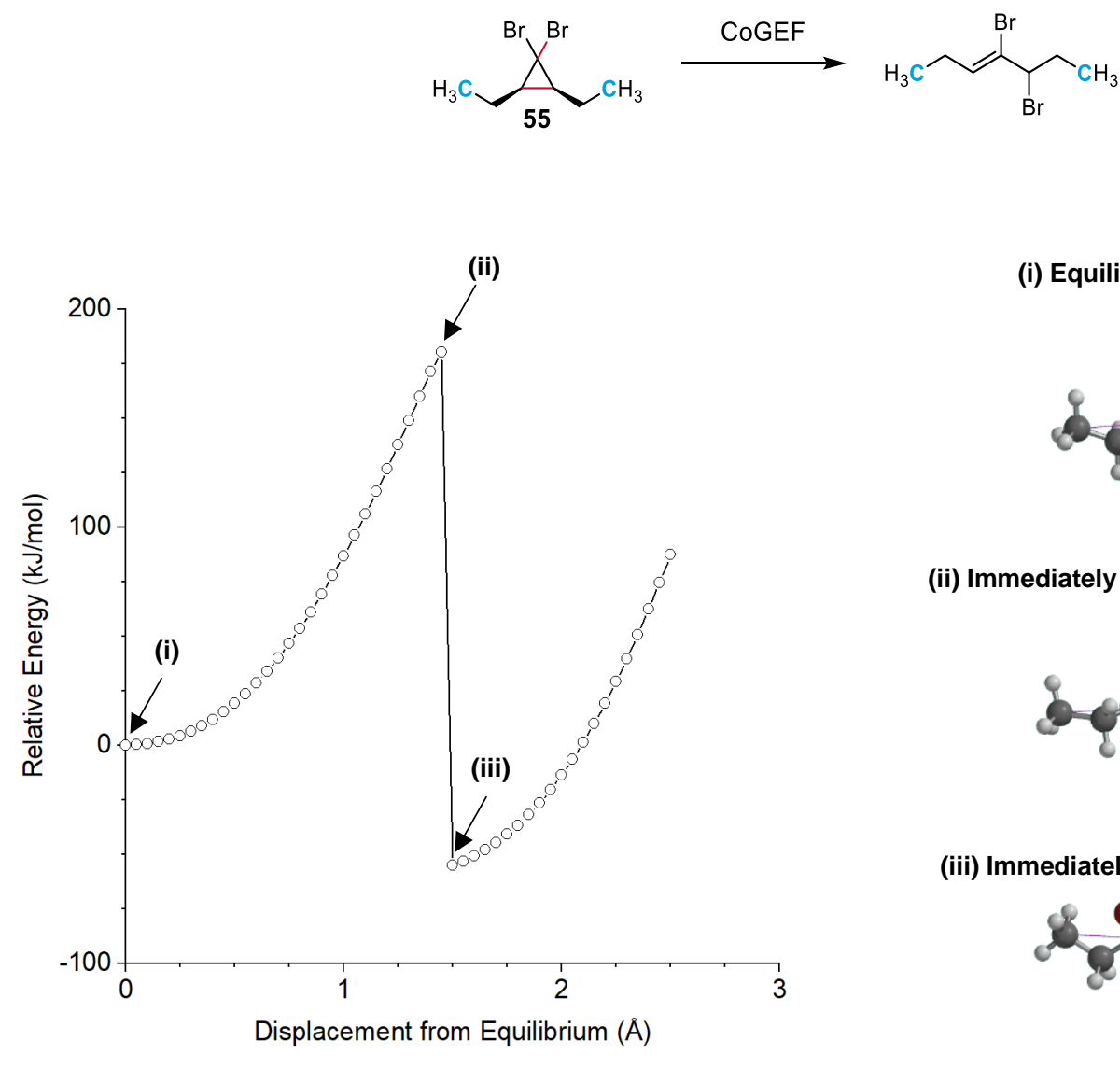

(i) Equilibrium Geometry

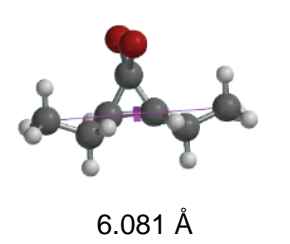

(ii) Immediately Prior to Bond Cleavage

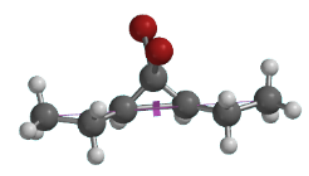

$7.531 \AA$

(iii) Immediately After Bond Cleavage

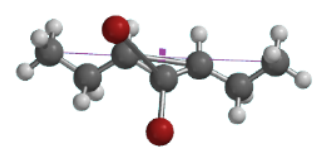

$7.581 \AA$

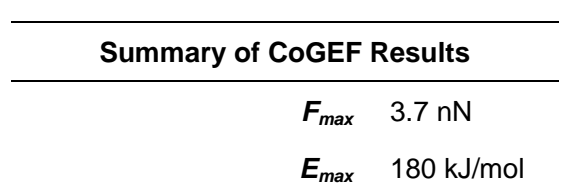

Force-Bond Angle $\quad 0.1^{\circ}$ 


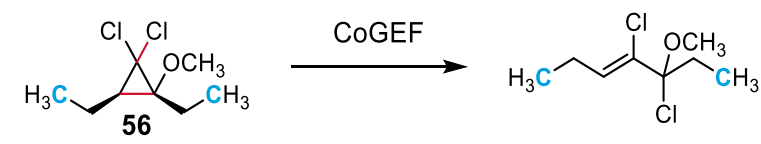

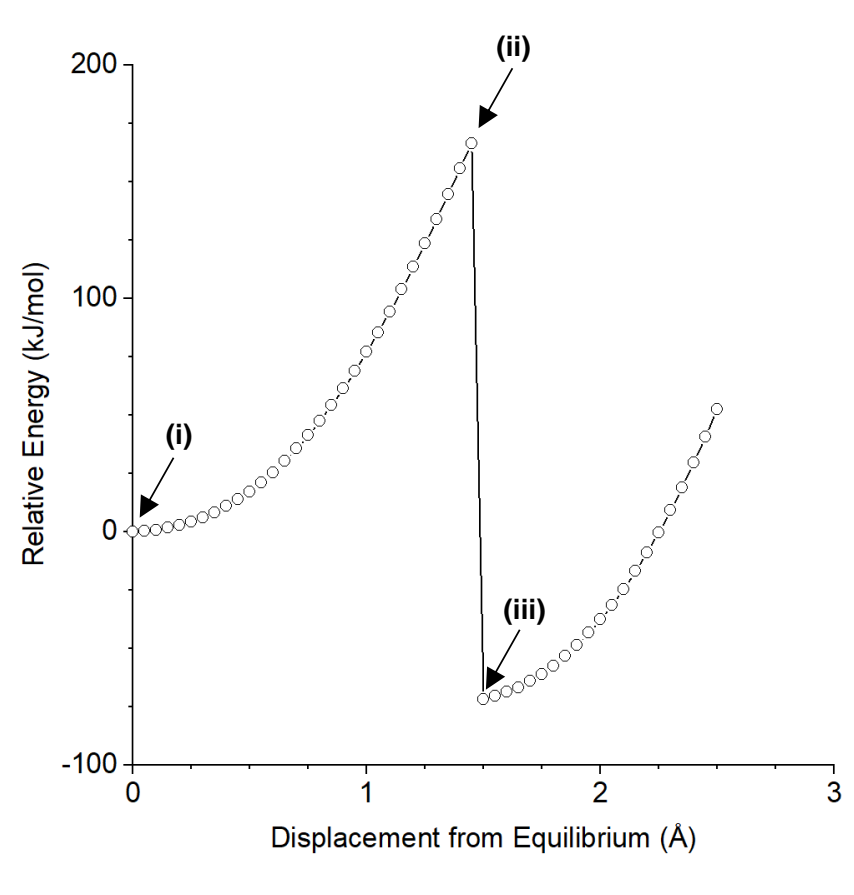

Summary of CoGEF Results

$$
\begin{array}{ll}
\boldsymbol{F}_{\text {max }} & 3.6 \mathrm{nN} \\
\boldsymbol{E}_{\max } & 166 \mathrm{~kJ} / \mathrm{mol}
\end{array}
$$

Force-Bond Angle $3.8^{\circ}$ (i) Equilibrium Geometry

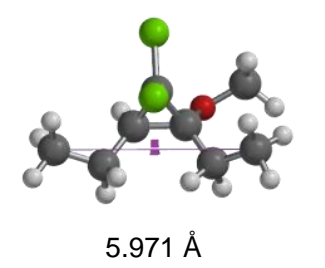

(ii) Immediately Prior to Bond Cleavage

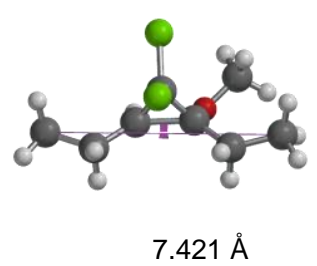

(iii) Immediately After Bond Cleavage

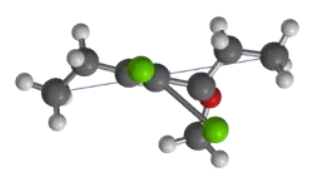

$7.471 \AA$ 

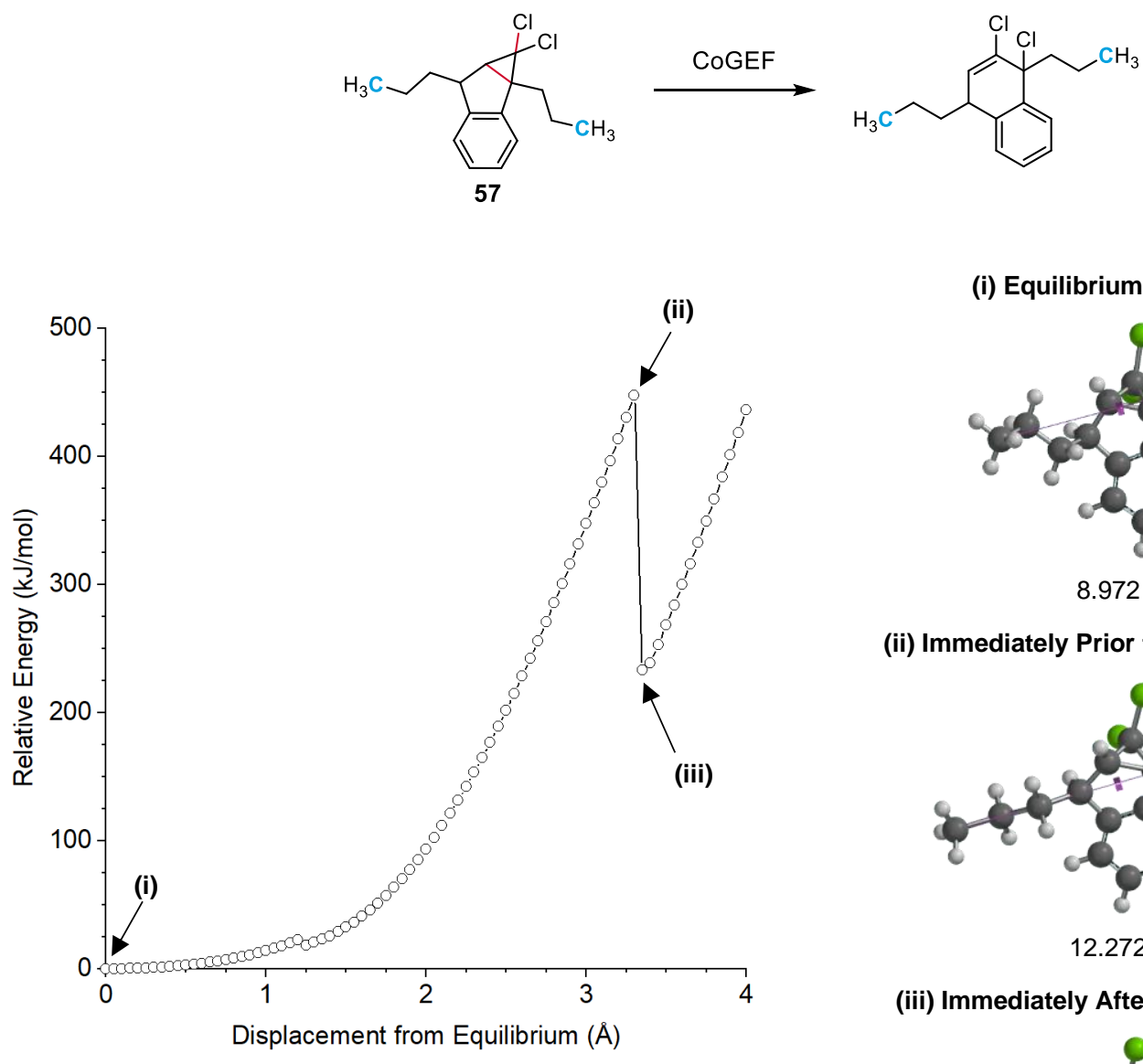

(i) Equilibrium Geometry

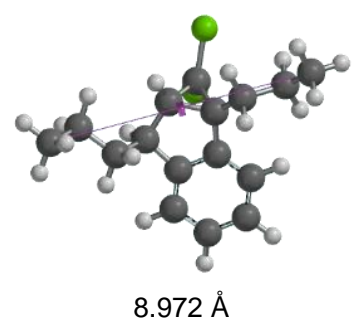

(ii) Immediately Prior to Bond Cleavage

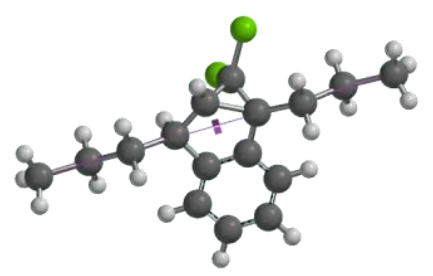

$12.272 \AA$

(iii) Immediately After Bond Cleavage

Summary of CoGEF Results

$$
\begin{array}{ll}
\boldsymbol{F}_{\max } & 5.7 \mathrm{nN} \\
\boldsymbol{E}_{\max } & 448 \mathrm{~kJ} / \mathrm{mol}
\end{array}
$$

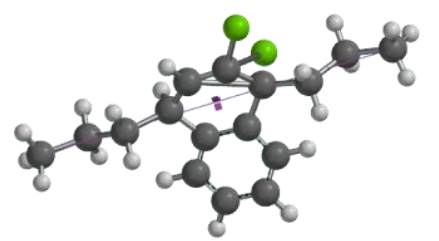

$12.322 \AA$ 

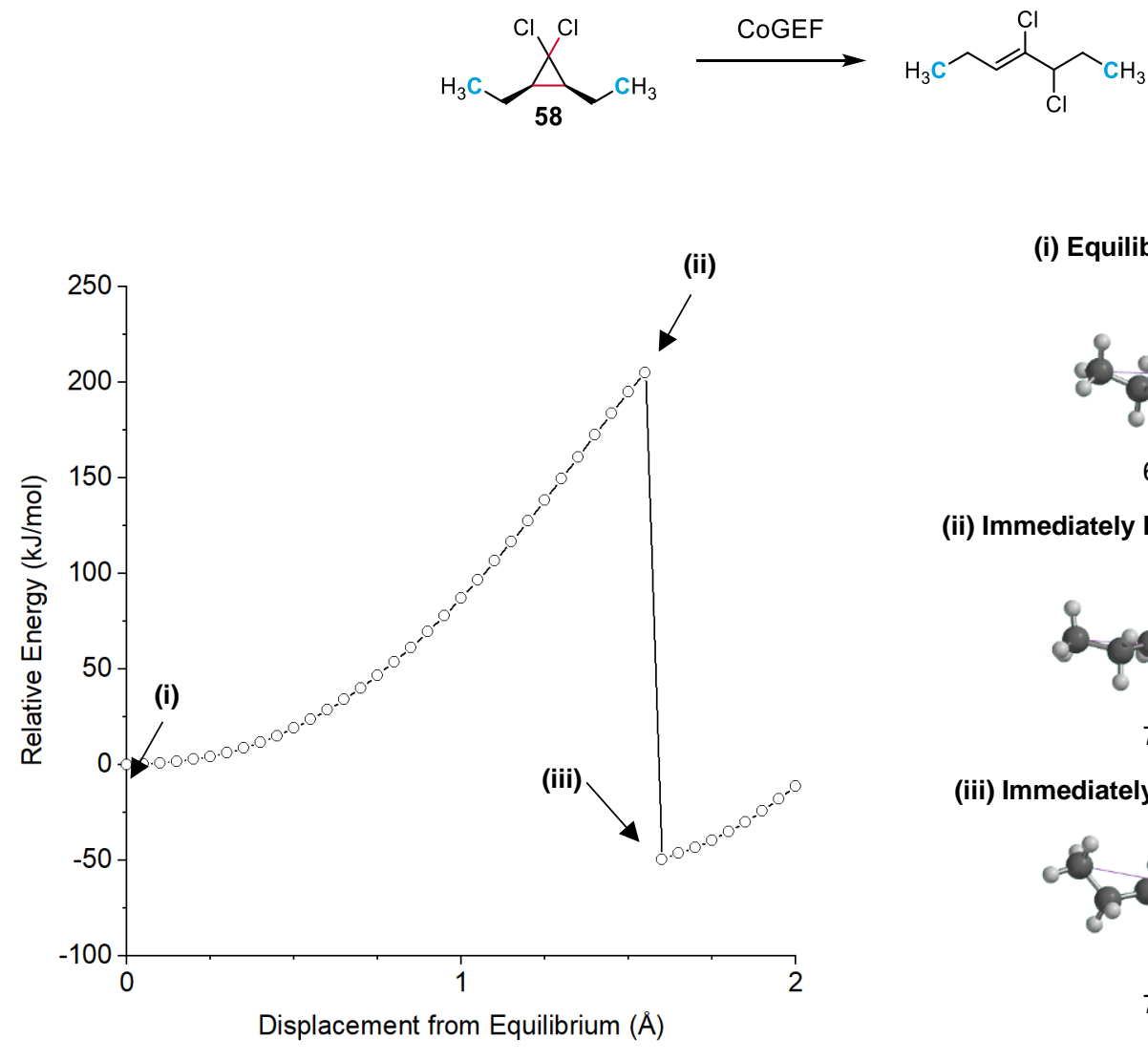

(i) Equilibrium Geometry

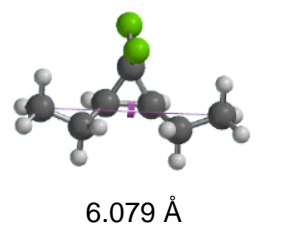

(ii) Immediately Prior to Bond Cleavage

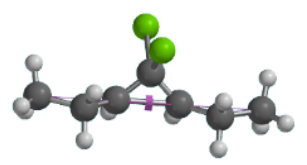

$7.629 \AA$

(iii) Immediately After Bond Cleavage

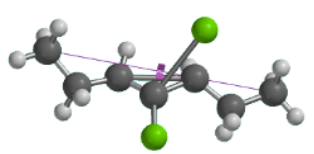

$7.679 \AA$

Summary of CoGEF Results

$$
\begin{array}{ll}
\boldsymbol{F}_{\text {max }} & 3.8 \mathrm{nN} \\
\boldsymbol{E}_{\max } & 205 \mathrm{~kJ} / \mathrm{mol}
\end{array}
$$

Force-Bond Angle $0.0^{\circ}$ 


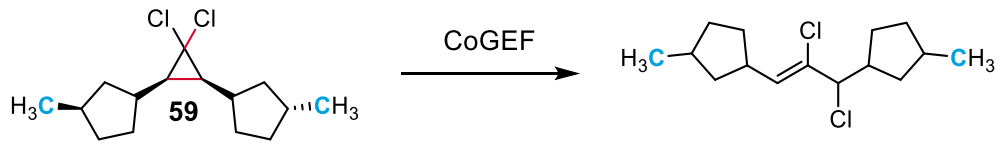

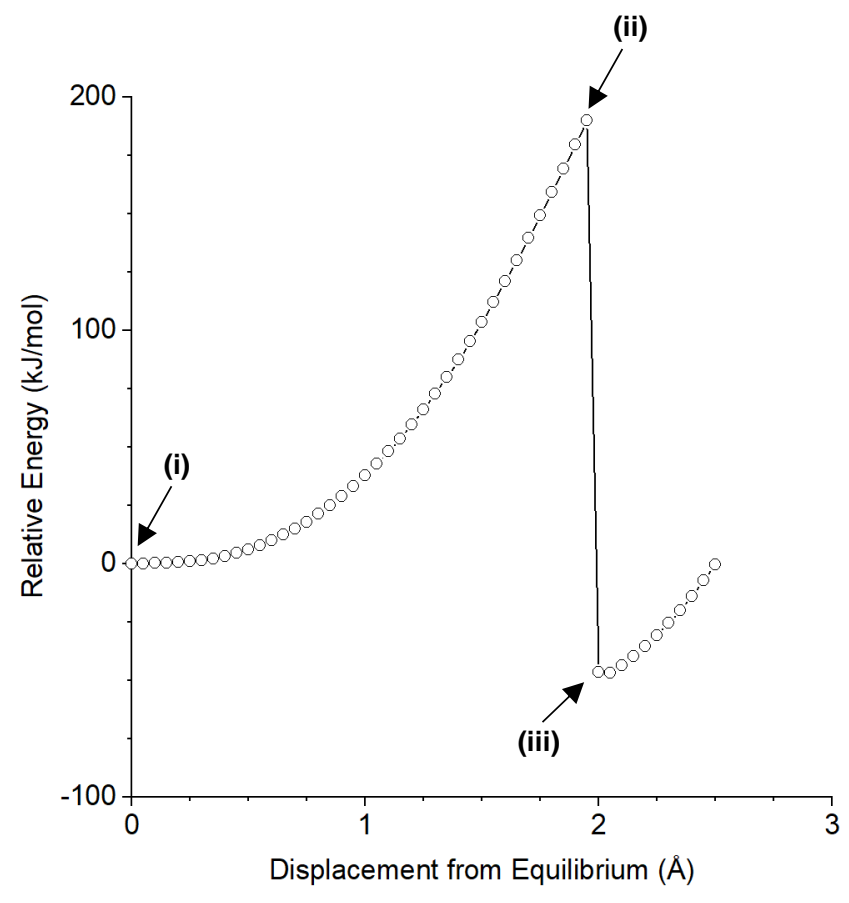

Summary of CoGEF Results

$$
\begin{array}{ll}
\boldsymbol{F}_{\text {max }} & 3.4 \mathrm{nN} \\
\boldsymbol{E}_{\text {max }} & 190 \mathrm{~kJ} / \mathrm{mol}
\end{array}
$$

Force-Bond Angle $\quad 0.4^{\circ}$

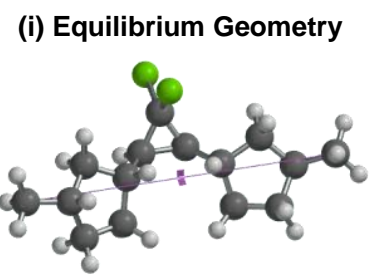

$10.746 \AA$

(ii) Immediately Prior to Bond Cleavage

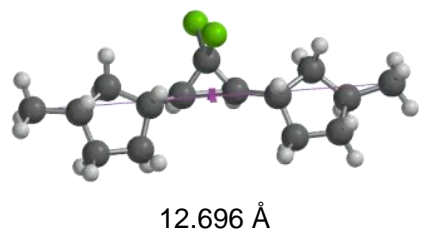

(iii) Immediately After Bond Cleavage

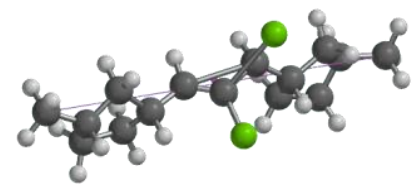

$12.746 \AA$ 


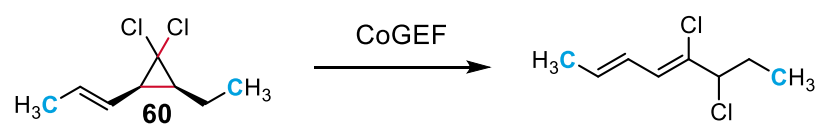

\section{(i) Equilibrium Geometry}
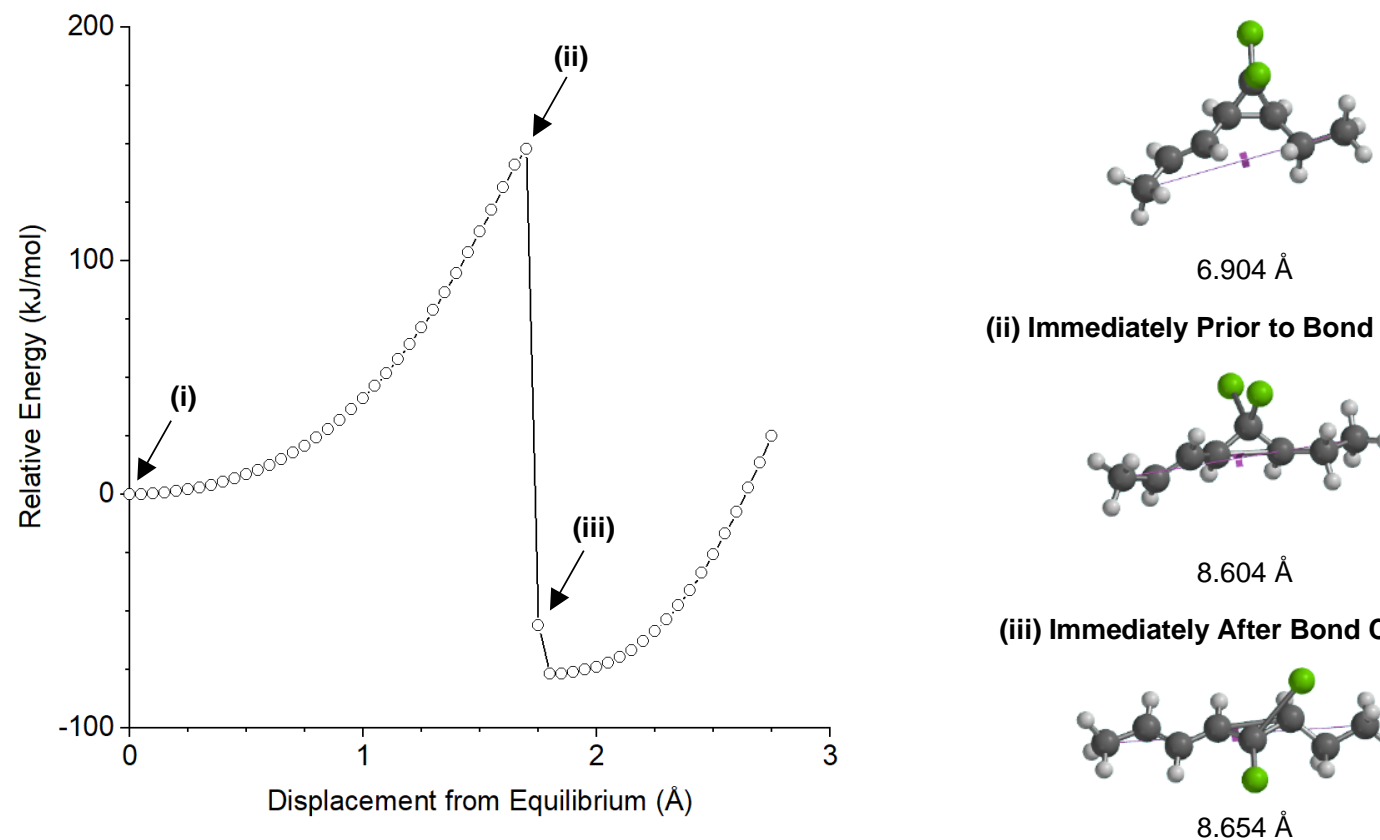

(ii) Immediately Prior to Bond Cleavage

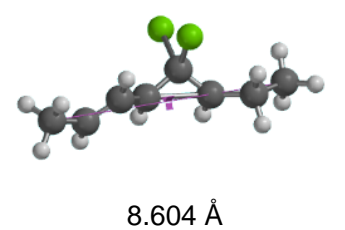

(iii) Immediately After Bond Cleavage

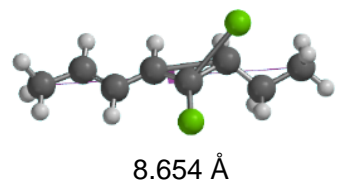

Summary of CoGEF Results

$$
\begin{array}{ll}
F_{\max } & 3.2 \mathrm{nN} \\
E_{\max } & 148 \mathrm{~kJ} / \mathrm{mol}
\end{array}
$$

Force-Bond Angle $9.0^{\circ}$ 


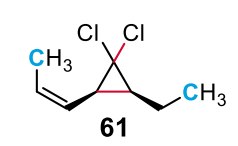

61

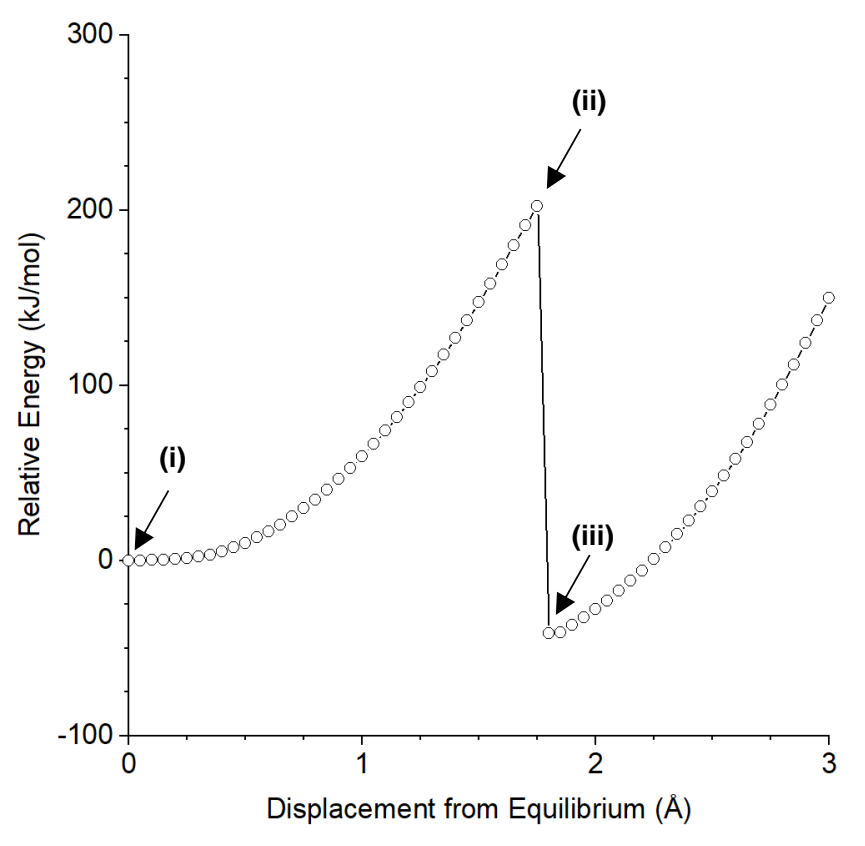

Summary of CoGEF Results

$\boldsymbol{F}_{\max } \quad 3.7 \mathrm{nN}$

$E_{\max } \quad 202 \mathrm{~kJ} / \mathrm{mol}$

Force-Bond Angle $3.9^{\circ}$ (i) Equilibrium Geometry

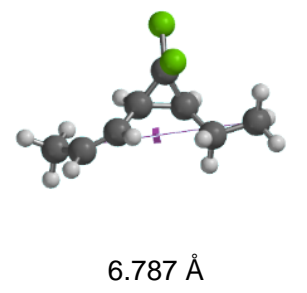

(ii) Immediately Prior to Bond Cleavage

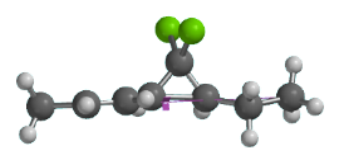

$8.537 \AA$

(iii) Immediately After Bond Cleavage

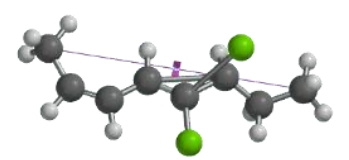

$8.587 \AA$ 

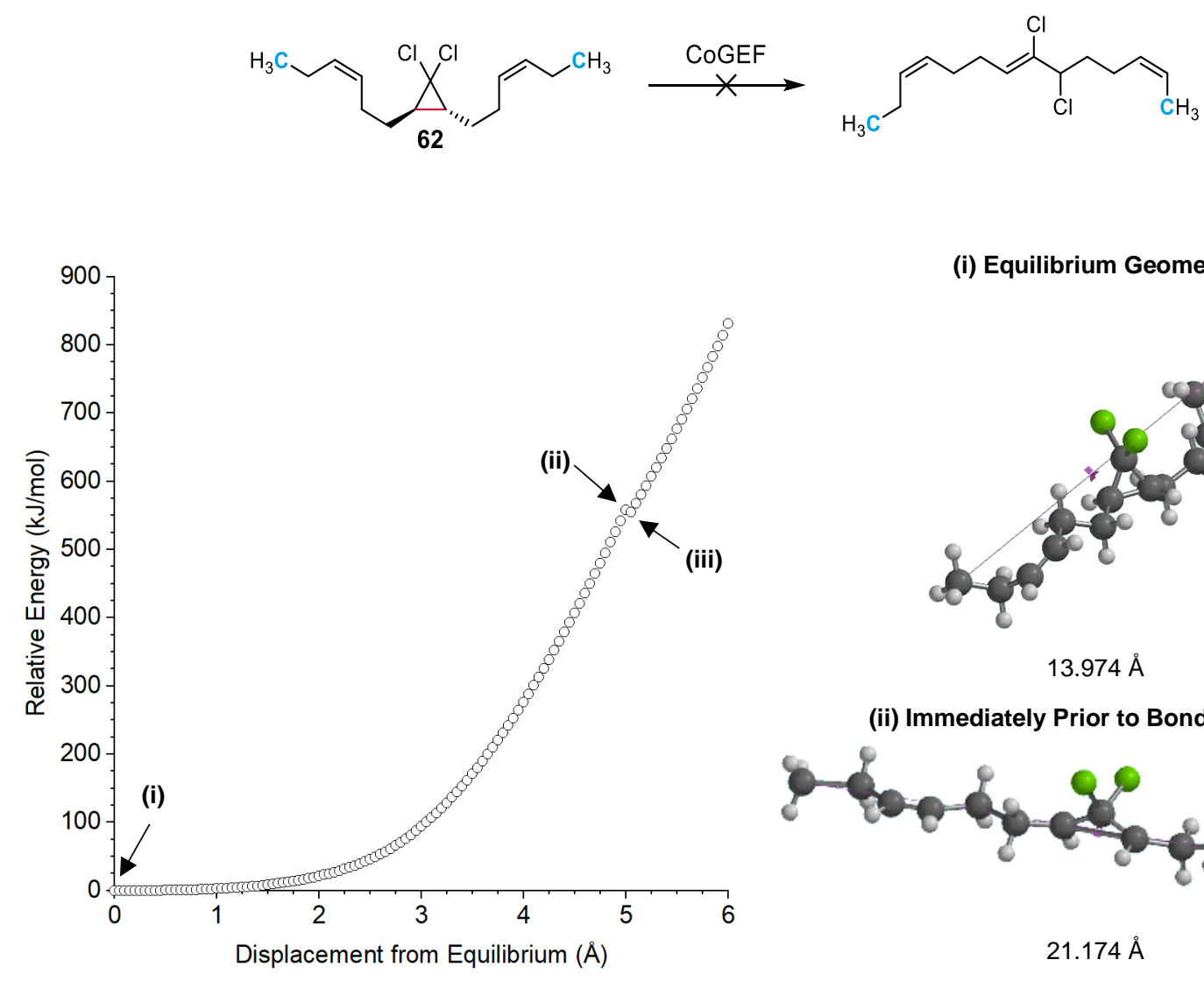

(i) Equilibrium Geometry

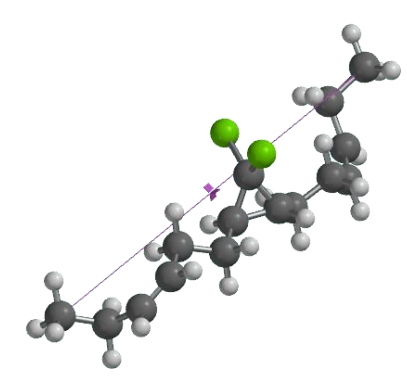

$13.974 \AA$

(ii) Immediately Prior to Bond Cleavage

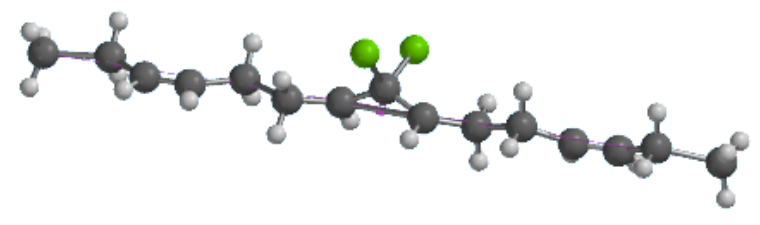

$21.174 \AA$

(iii) Immediately After Bond Cleavage

Summary of CoGEF Results

$\begin{array}{ll}\boldsymbol{F}_{\max } & 5.2 \mathrm{nN} \\ \boldsymbol{E}_{\max } & 557 \mathrm{~kJ} / \mathrm{mol}\end{array}$

Force-Bond Angle $20^{\circ}$

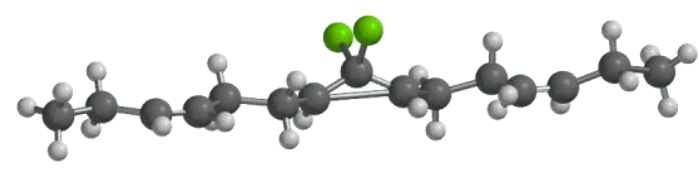

$21.224 \AA$ 

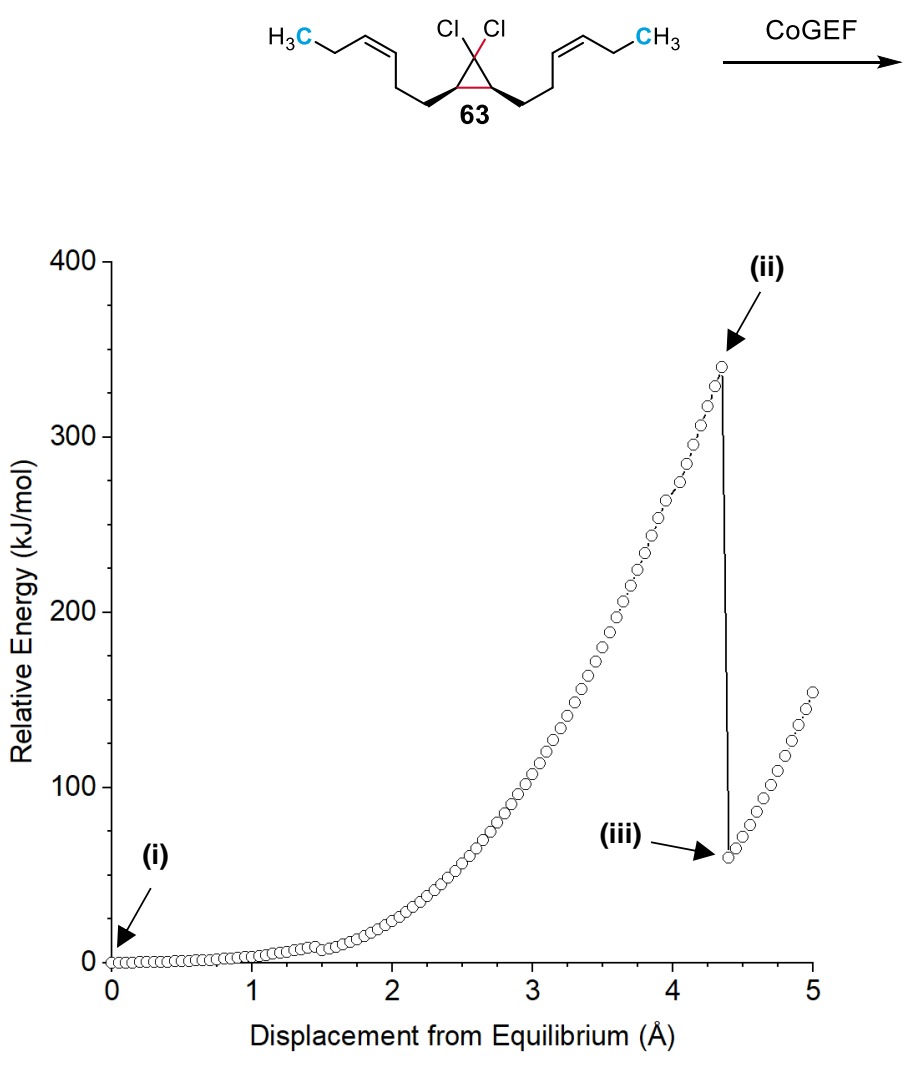

Force/Bond angle $\quad 0.1^{\circ}$

\section{(i) Equilibrium Geometry}

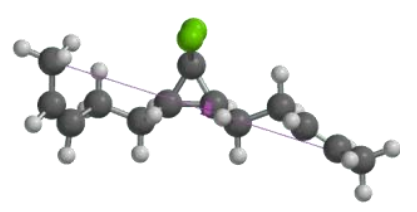

$11.037 \AA$

(i) Immediately Prior to Bond Cleavage

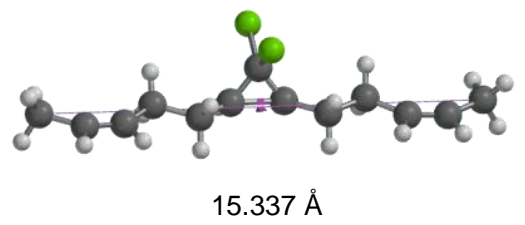

(ii) Immediately After Bond Cleavage

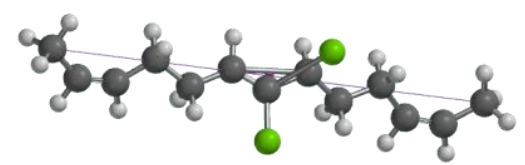

$15.387 \AA$

Summary of CoGEF Results

$$
\begin{array}{ll}
\boldsymbol{F}_{\text {max }} & 3.8 \mathrm{nN} \\
\boldsymbol{E}_{\text {max }} & 340 \mathrm{~kJ} / \mathrm{mol}
\end{array}
$$


<smiles>COC(=O)C(C(=O)OC)N(c1ccc(OC)cc1)C(C(=O)OC)C(=O)OC</smiles>

(i) Equilibrium Geometry

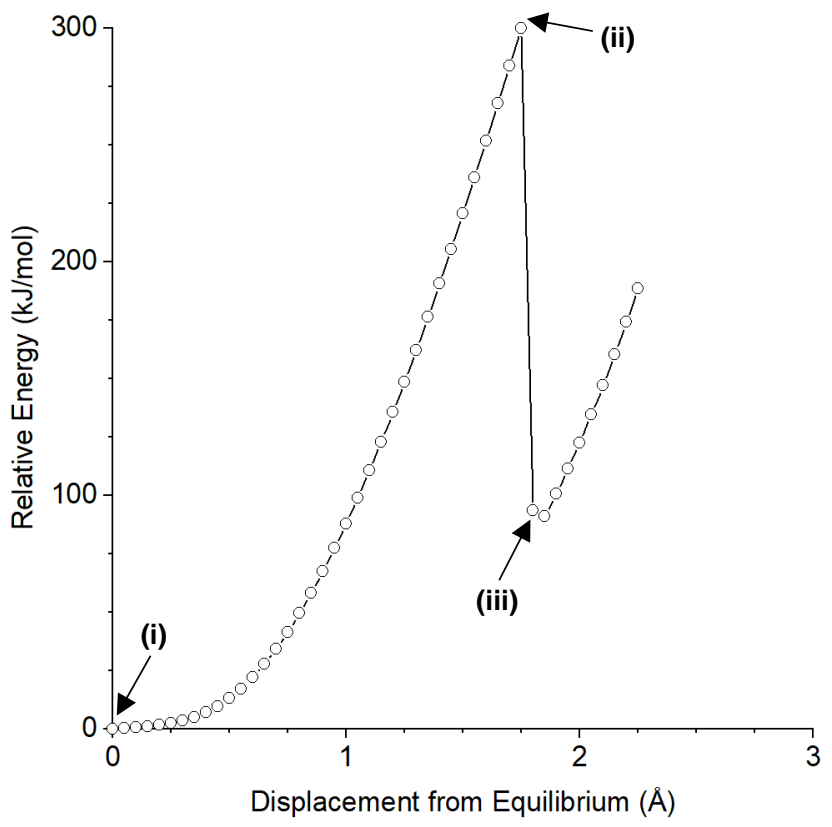

Summary of CoGEF Results

$\boldsymbol{F}_{\max } \quad 5.4 \mathrm{nN}$

$E_{\max } \quad 300 \mathrm{~kJ} / \mathrm{mol}$

Force-Bond Angle $22^{\circ}$

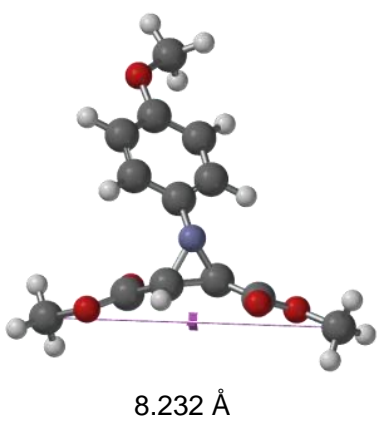

(ii) Immediately Prior to Bond Cleavage

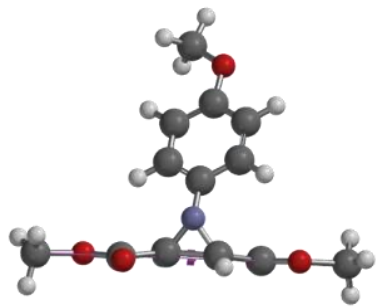

$9.982 \AA$

(iii) Immediately After Bond Cleavage

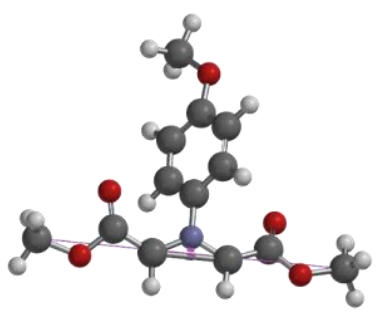

$10.032 \AA$ 


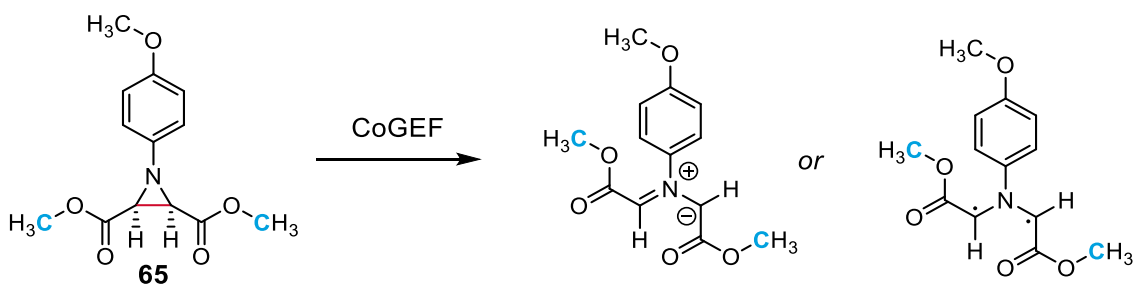

(i) Equilibrium Geometry

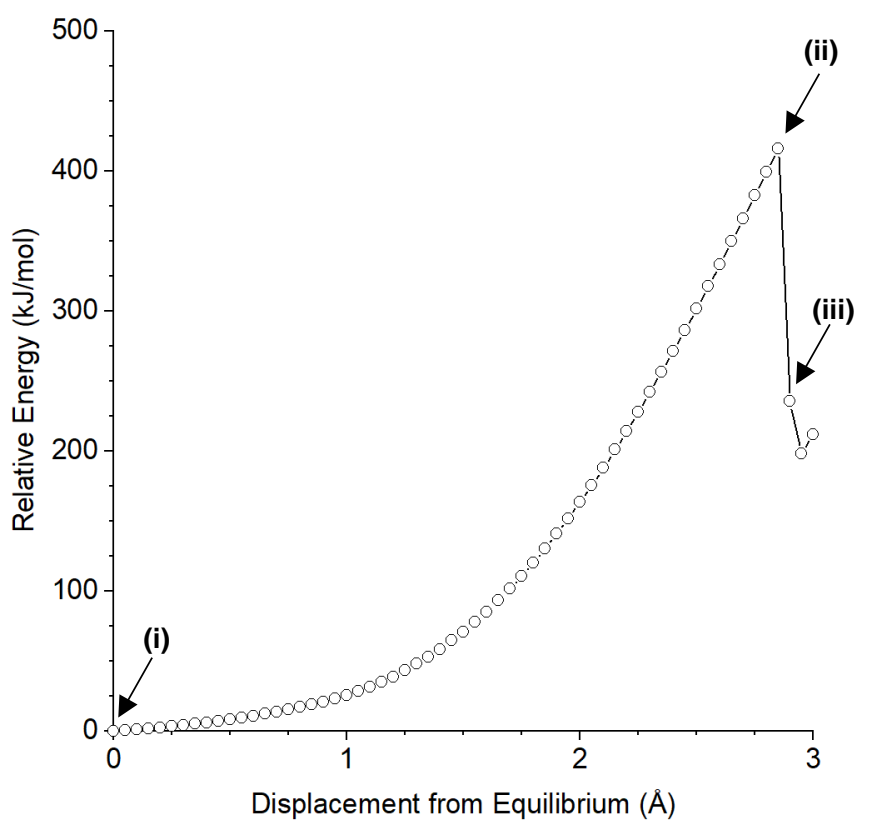

Summary of CoGEF Results

$F_{\max } \quad 5.6 \mathrm{nN}$

$E_{\max } \quad 416 \mathrm{~kJ} / \mathrm{mol}$

Force-Bond Angle $\quad 0.1^{\circ}$

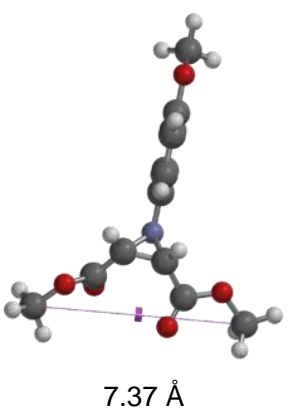

(ii) Immediately Prior to Bond Cleavage

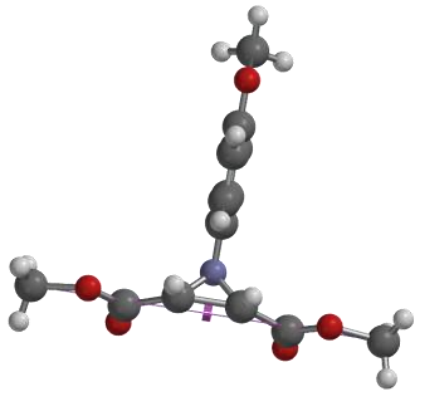

$10.22 \AA$

(iii) Immediately After Bond Cleavage

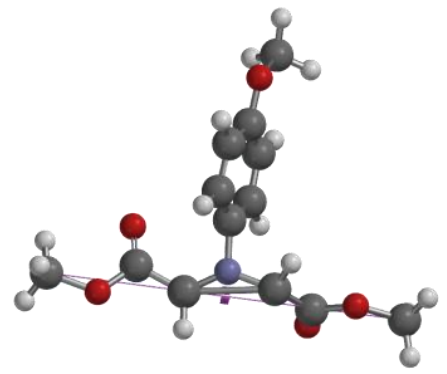

$10.27 \AA$ 
<smiles>CC1CCC(C2OC2C2CC[C@@H](C)C2)C1</smiles>

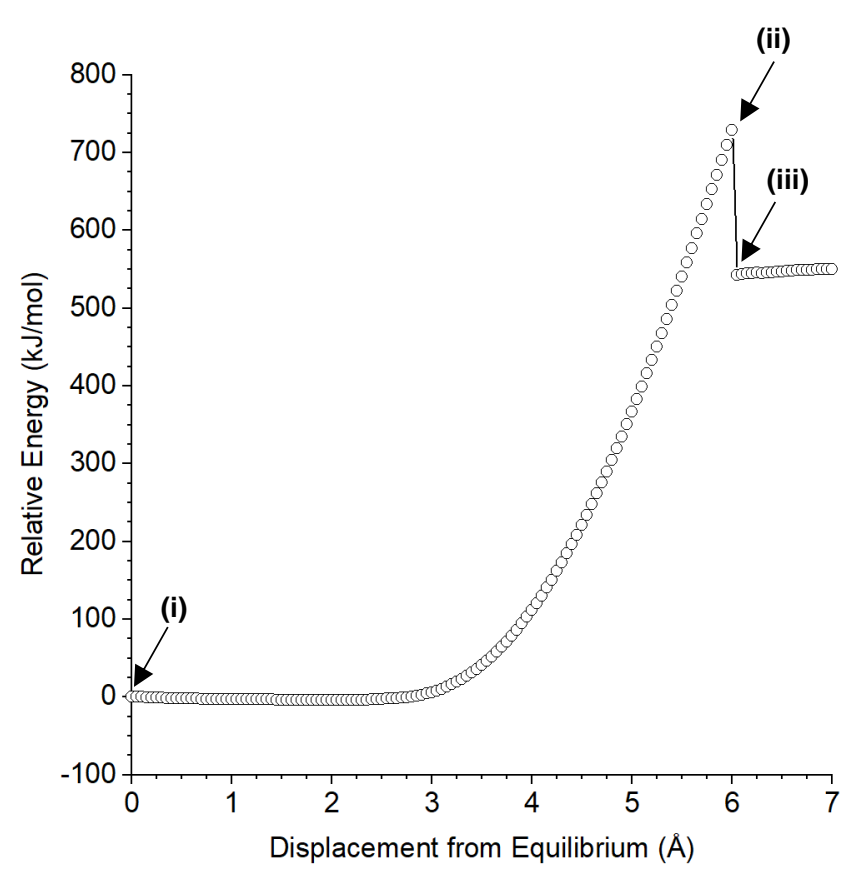

(i) Equilibrium Geometry

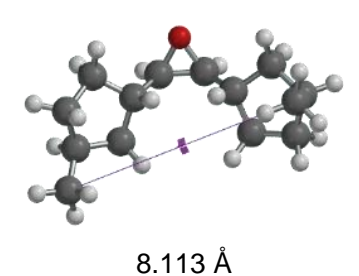

$8.113 \AA$

(ii) Immediately Prior to Bond Cleavage

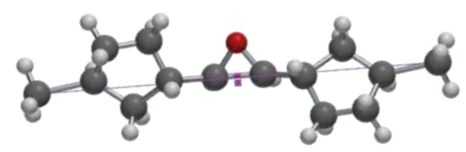

$14.113 \AA$

(iii) Immediately After Bond Cleavage

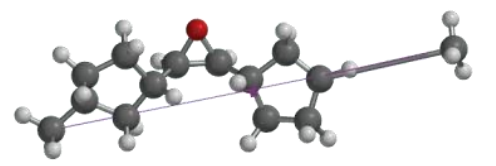

$14.163 \AA$

Summary of CoGEF Results

$\boldsymbol{F}_{\max } \quad 6.4 \mathrm{nN}$

$E_{\max } 729 \mathrm{~kJ} / \mathrm{mol}$

Force-Bond Angle $\quad 0.8^{\circ}$ 
<smiles>C/C=C/B1OC1CCC</smiles>

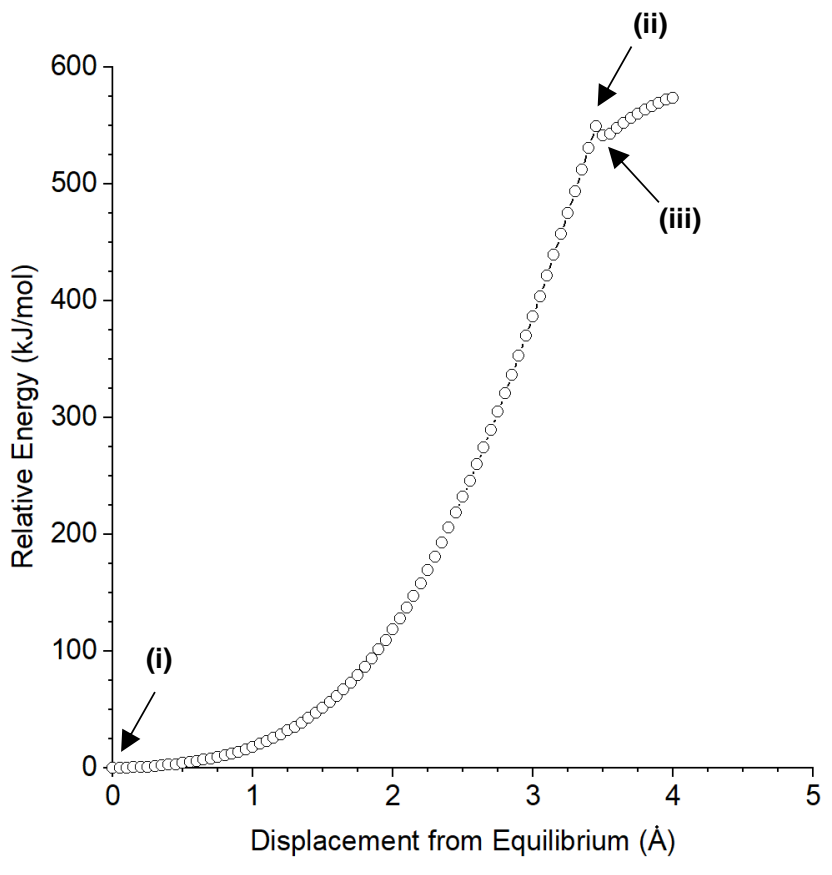

Summary of CoGEF Results

$$
\begin{array}{ll}
\boldsymbol{F}_{\max } & 6.2 \mathrm{nN} \\
\boldsymbol{E}_{\max } & 549 \mathrm{~kJ} / \mathrm{mol}
\end{array}
$$

Force-Bond Angle $1.5^{\circ}$
CoGEF $X$ $\mathrm{H}_{3} \mathrm{C}$

(i) Equilibrium Geometry

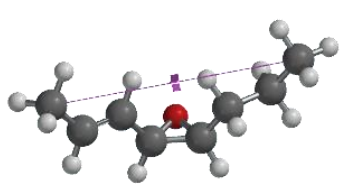

$7.555 \AA$

(ii) Immediately Prior to Bond Cleavage

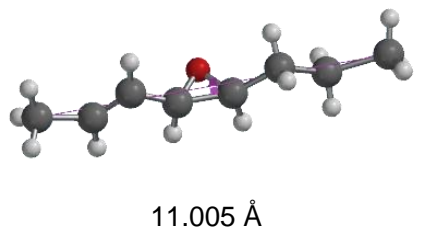

(iii) Immediately After Bond Cleavage

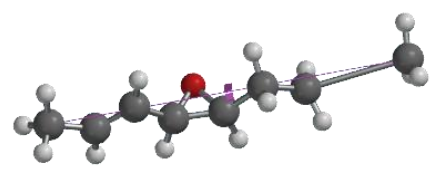

$11.055 \AA$ 

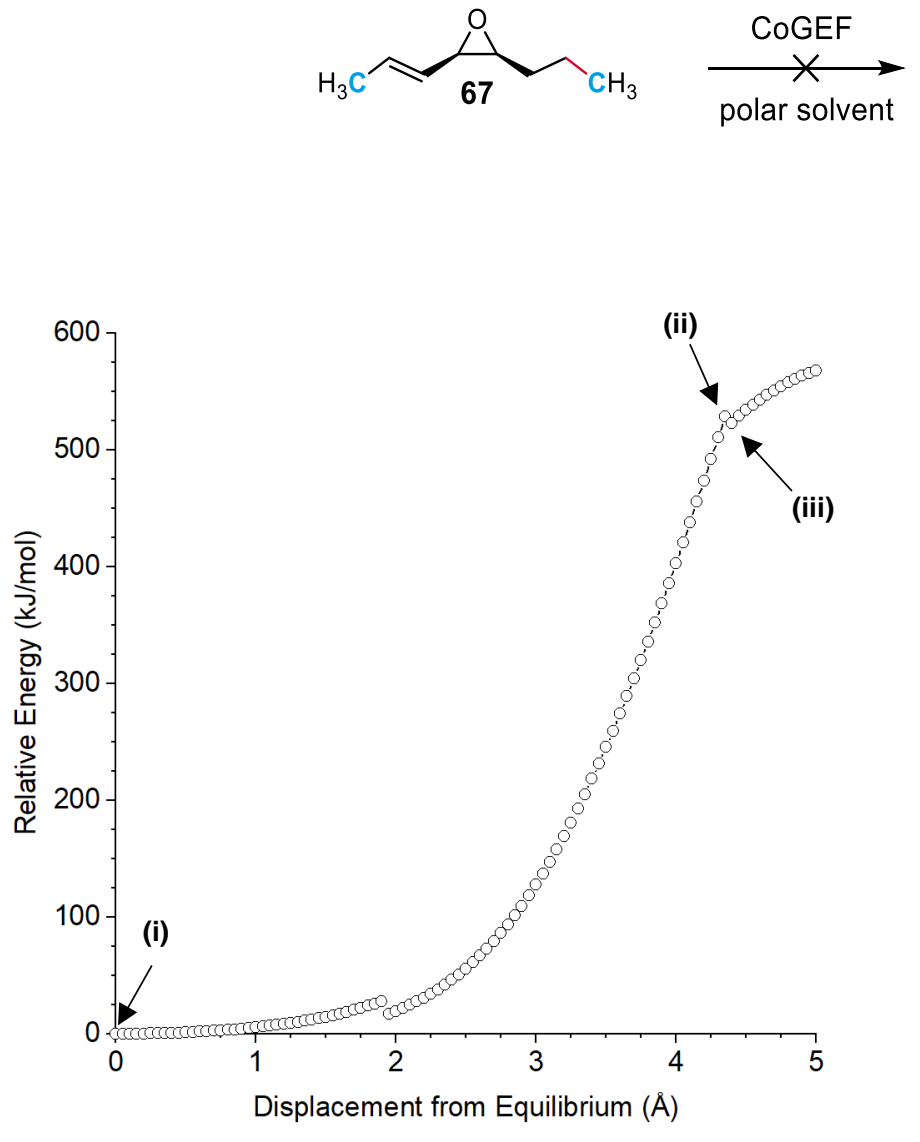

Summary of CoGEF Results

$\begin{array}{ll}\boldsymbol{F}_{\max } & 6.1 \mathrm{nN} \\ \boldsymbol{E}_{\max } & 529 \mathrm{~kJ} / \mathrm{mol}\end{array}$

Force-Bond Angle $1.6^{\circ}$<smiles></smiles>

(i) Equilibrium Geometry

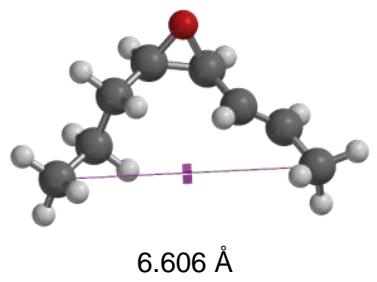

(ii) Immediately Prior to Bond Cleavage

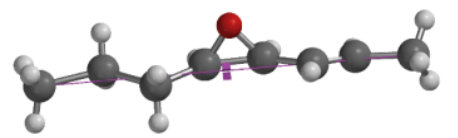

$10.956 \AA$

(iii) Immediately After Bond Cleavage

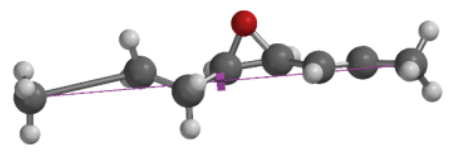

$11.006 \AA$ 

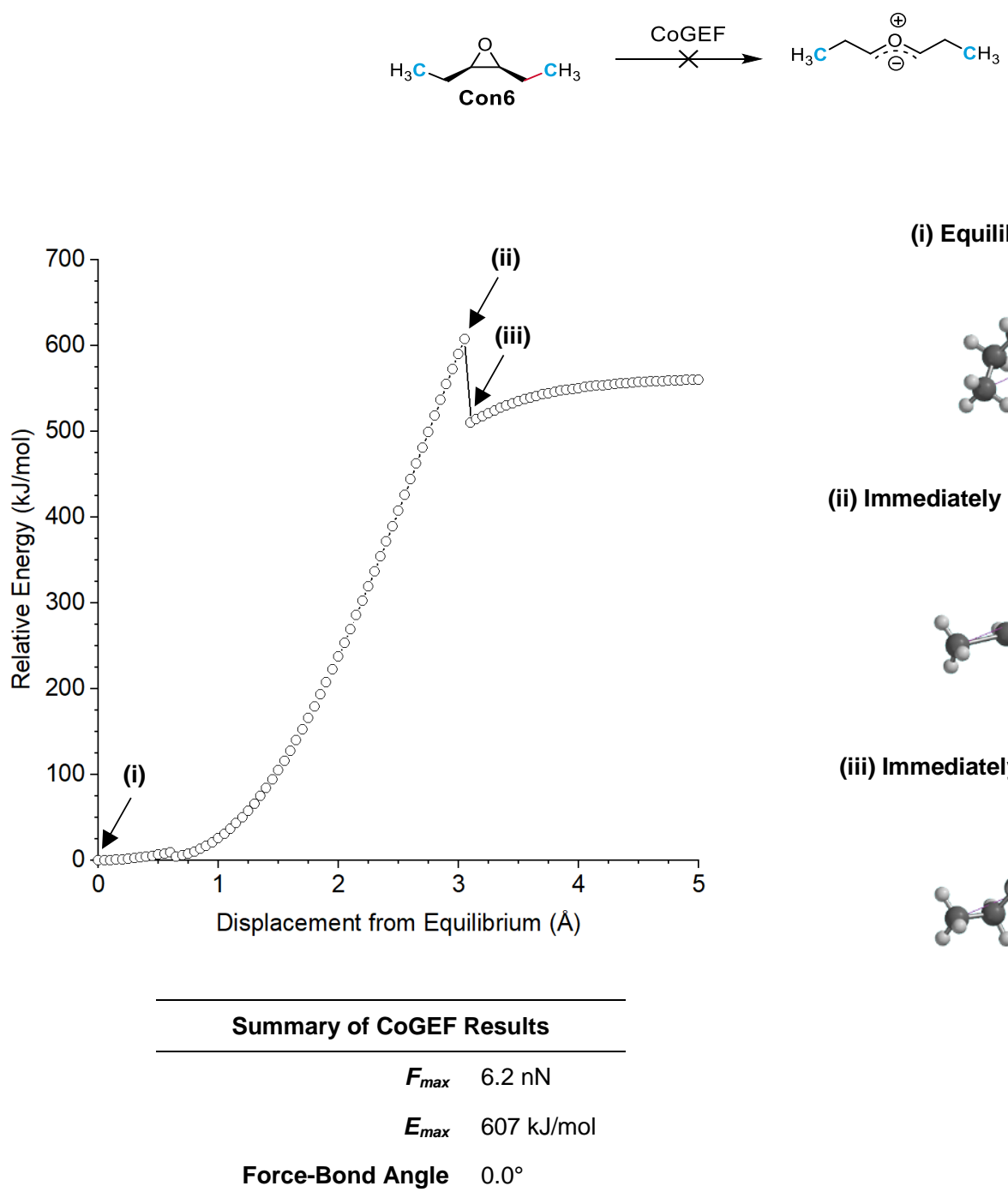

(i) Equilibrium Geometry

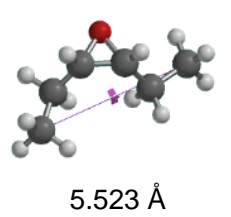

(ii) Immediately Prior to Bond Cleavage

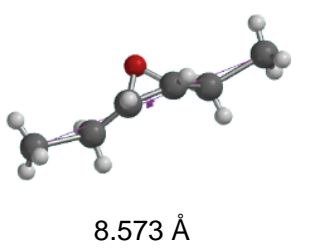

(iii) Immediately After Bond Cleavage

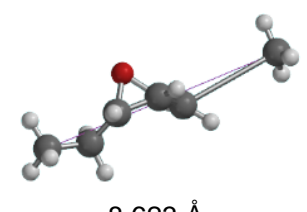

$8.623 \AA$

Force-Bond Angle $\quad 0.0^{\circ}$ 

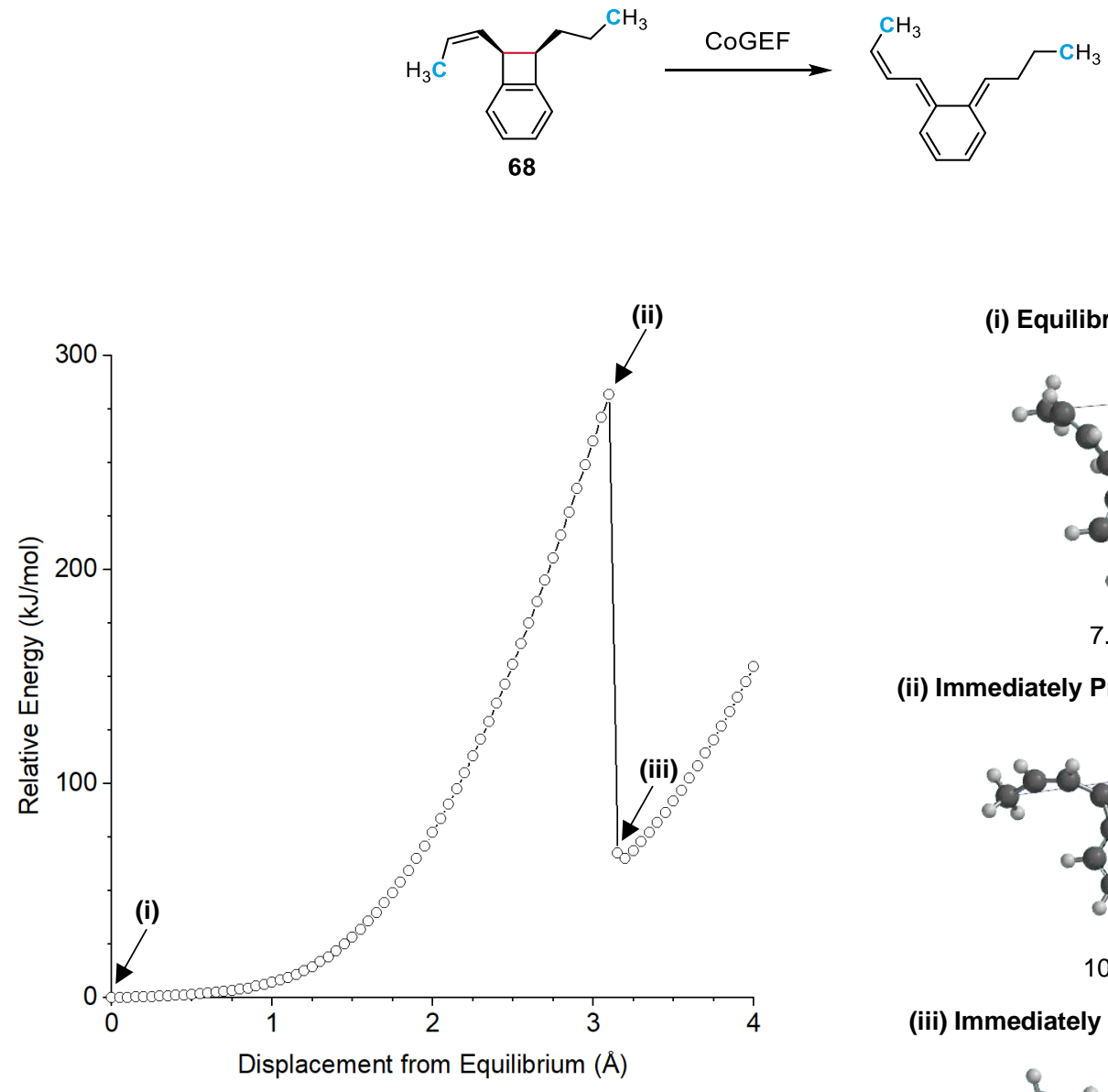

(i) Equilibrium Geometry

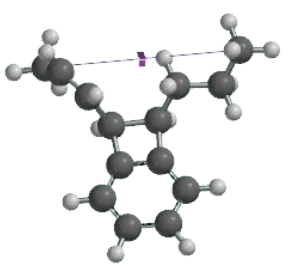

$7.067 \AA$

(ii) Immediately Prior to Bond Cleavage

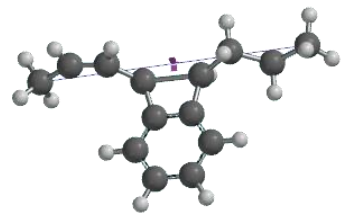

$10.167 \AA$

(iii) Immediately After Bond Cleavage

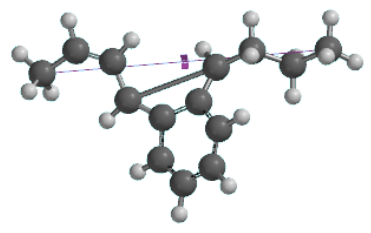

$10.217 \AA$

Force/Bond angle $3.2^{\circ}$ 


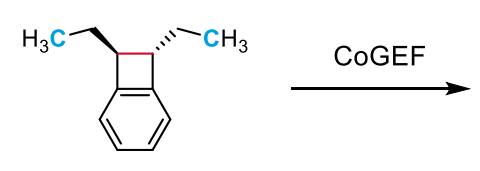

69

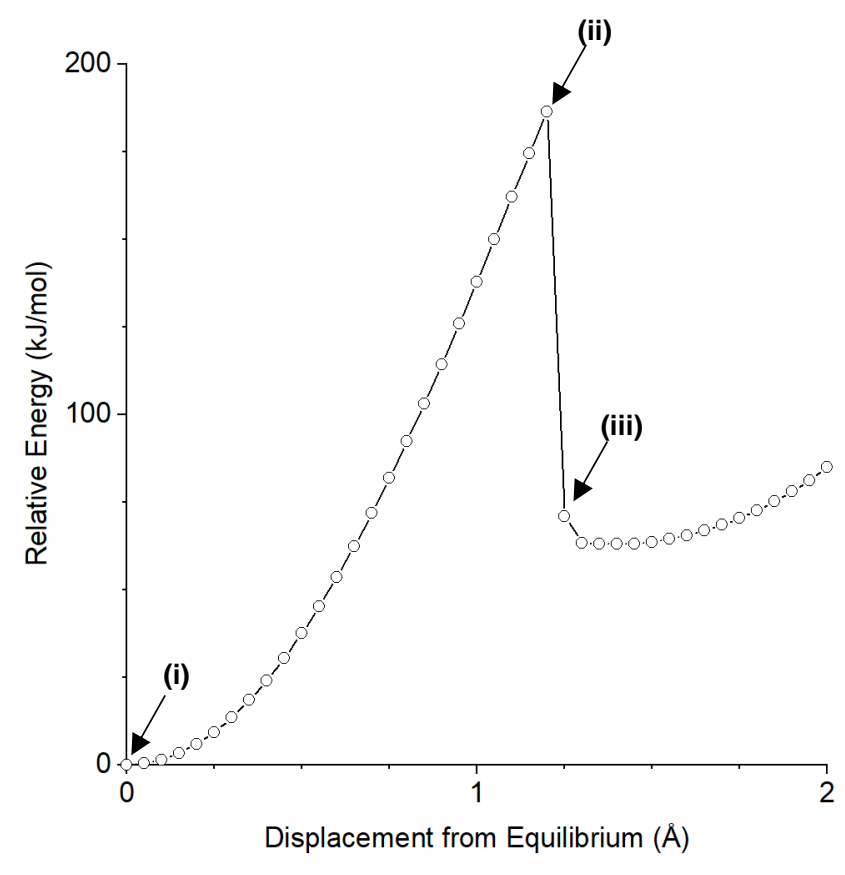

Summary of CoGEF Results

$$
\begin{array}{ll}
\boldsymbol{F}_{\max } & 4.1 \mathrm{nN} \\
\boldsymbol{E}_{\max } & 186 \mathrm{~kJ} / \mathrm{mol}
\end{array}
$$

Force/Bond angle $17^{\circ}$

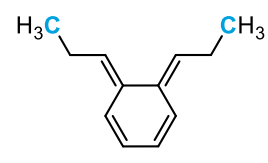

(i) Equilibrium Geometry

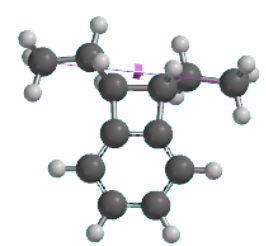

$6.463 \AA$

(ii) Immediately Prior to Bond Cleavage

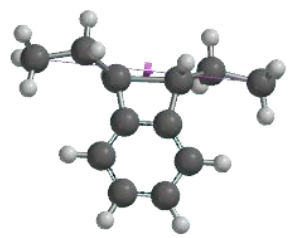

$7.663 \AA$

(iii) Immediately After Bond Cleavage

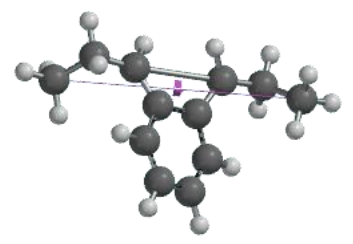

$7.713 \AA$ 


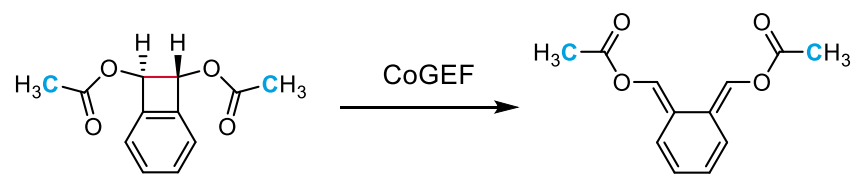

70

(i) Equilibrium Geometry
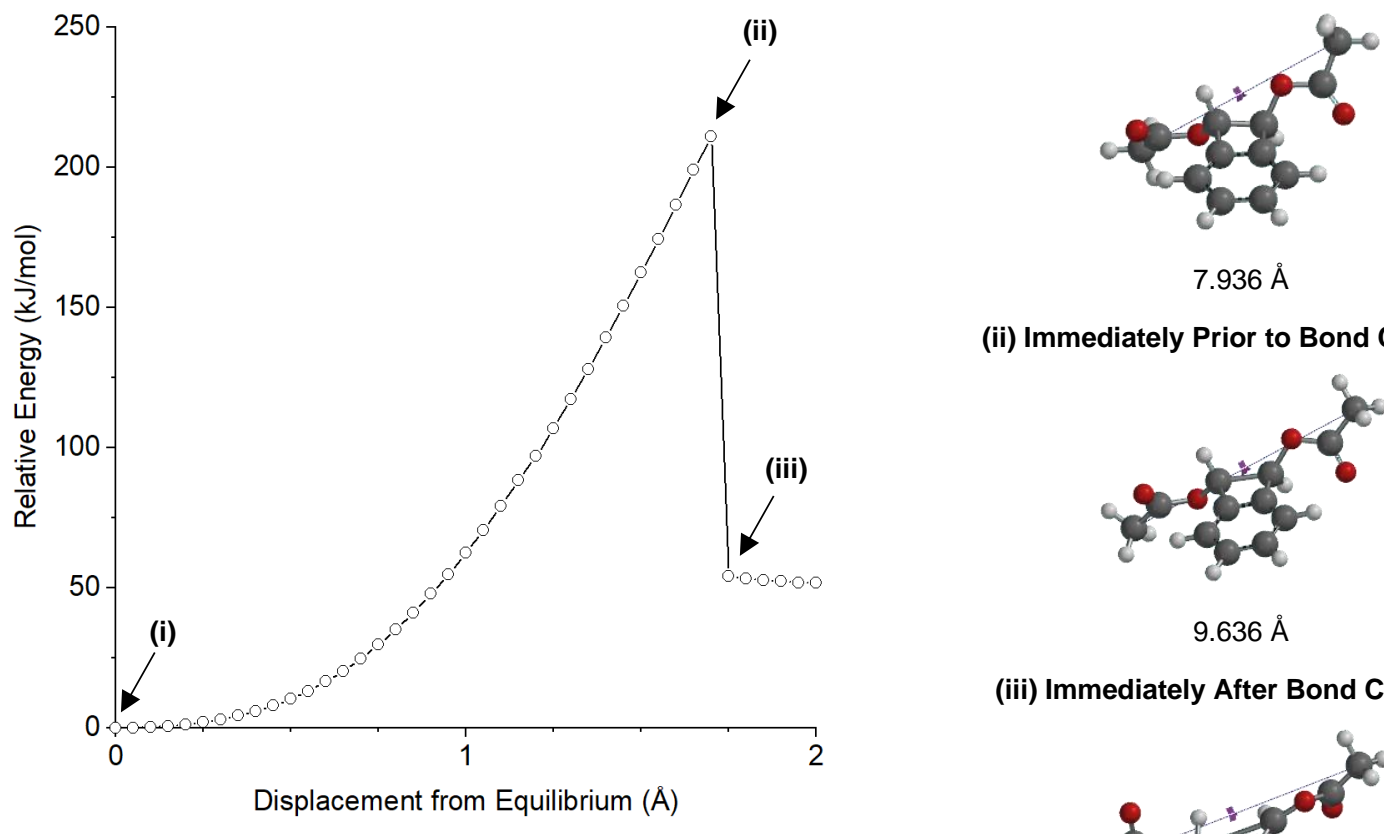

$7.936 \AA$

(ii) Immediately Prior to Bond Cleavage

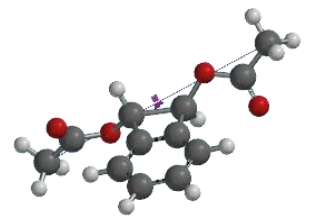

$9.636 \AA$

(iii) Immediately After Bond Cleavage

Summary of CoGEF Results

$$
\begin{array}{ll}
\boldsymbol{F}_{\max } & 4.1 \mathrm{nN} \\
\boldsymbol{E}_{\max } & 211 \mathrm{~kJ} / \mathrm{mol}
\end{array}
$$

Force-Bond Angle $25^{\circ}$

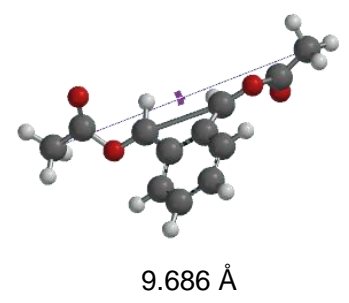




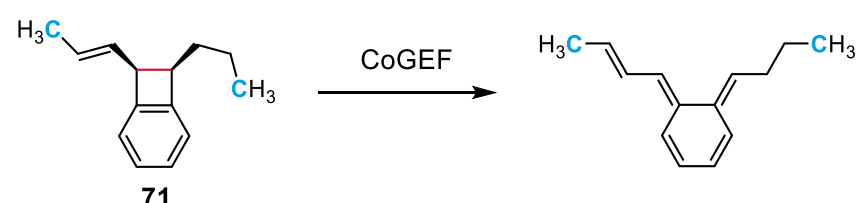

(i) Equilibrium Geometry
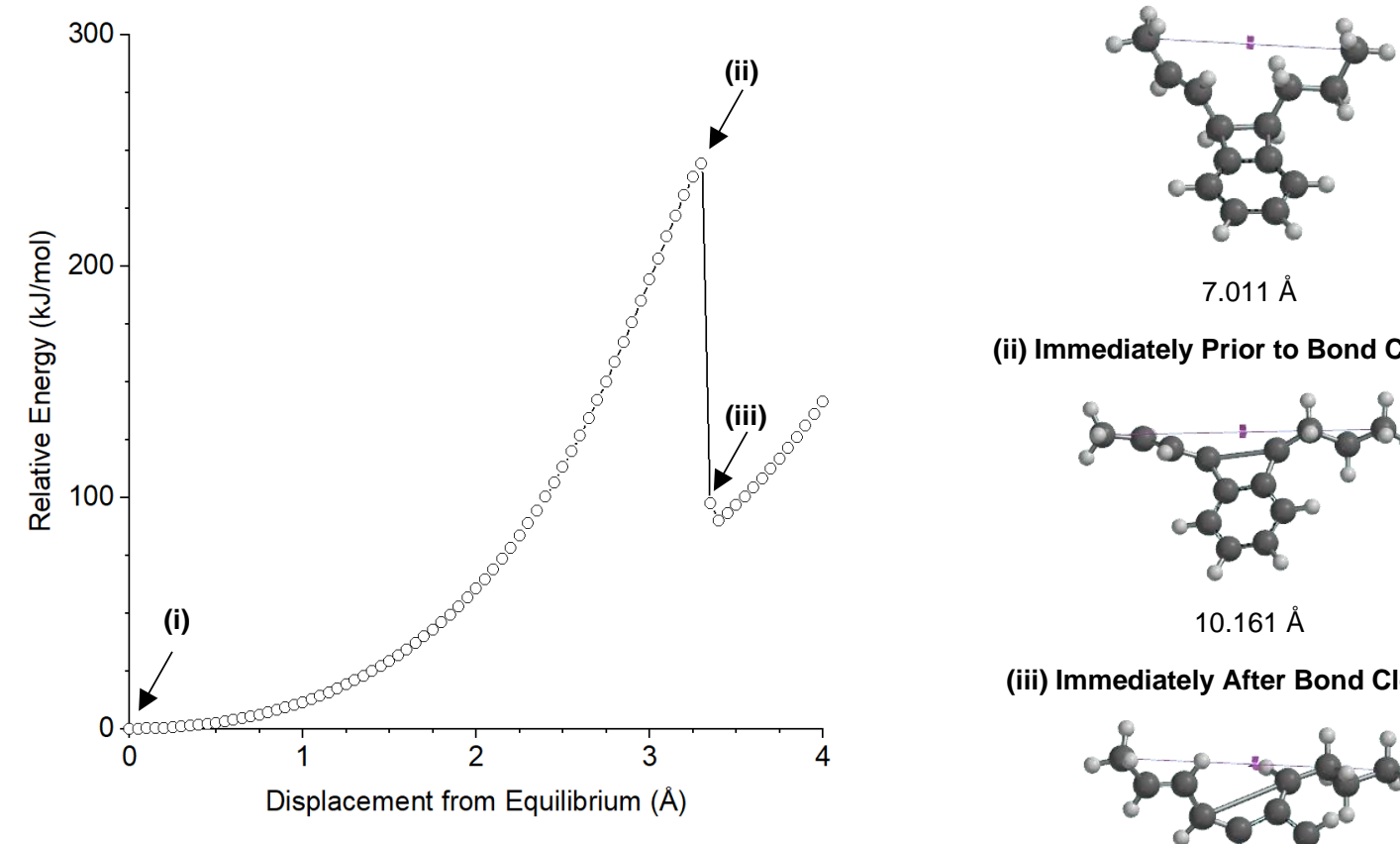

$7.011 \AA$

(ii) Immediately Prior to Bond Cleavage

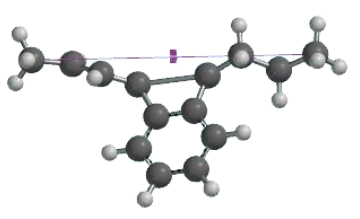

$10.161 \AA$

(iii) Immediately After Bond Cleavage

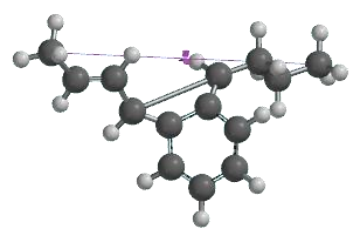

Summary of CoGEF Results

$10.211 \AA$

$F_{\max } \quad 3.1 \mathrm{nN}$

$E_{\max } 244 \mathrm{~kJ} / \mathrm{mol}$

Force-Bond Angle $2.8^{\circ}$ 


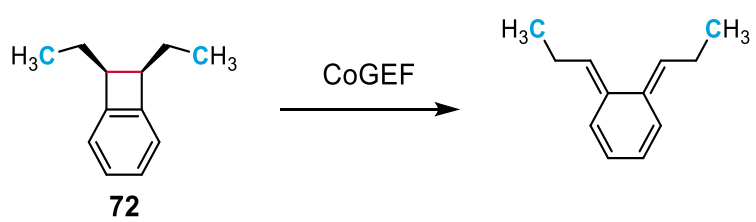

(i) Equilibrium Geometry

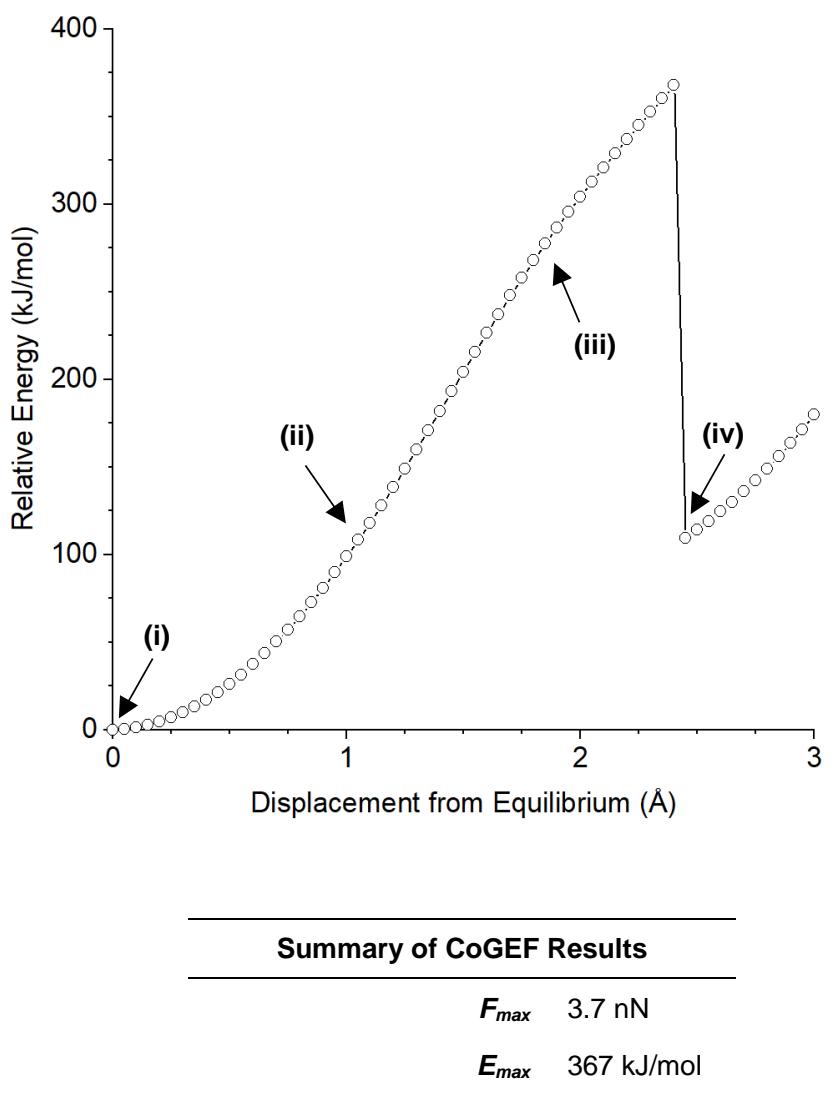

Force/Bond angle $\quad 0.0^{\circ}$

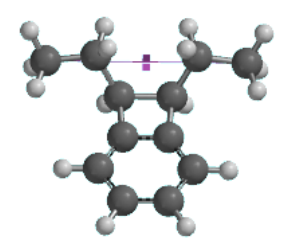

$6.123 \AA$

(ii) Prior to Bond Cleavage

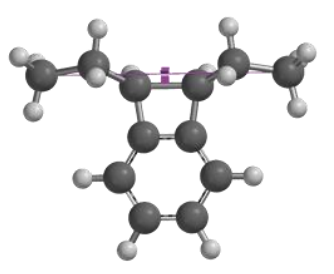

$7.273 \AA$

(iii) After Bond Cleavage

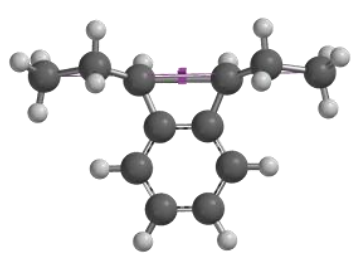

8.123

(iv) After Formation of Double Bonds

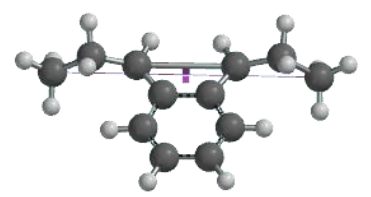

$8.573 \AA$ 


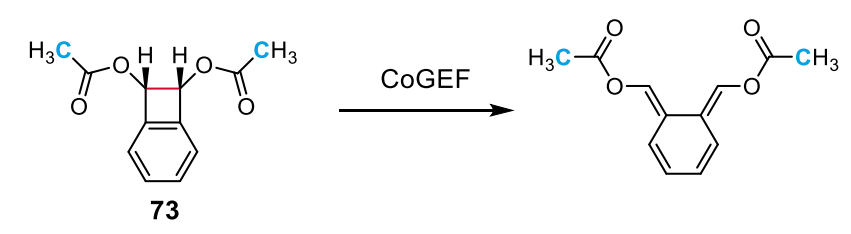

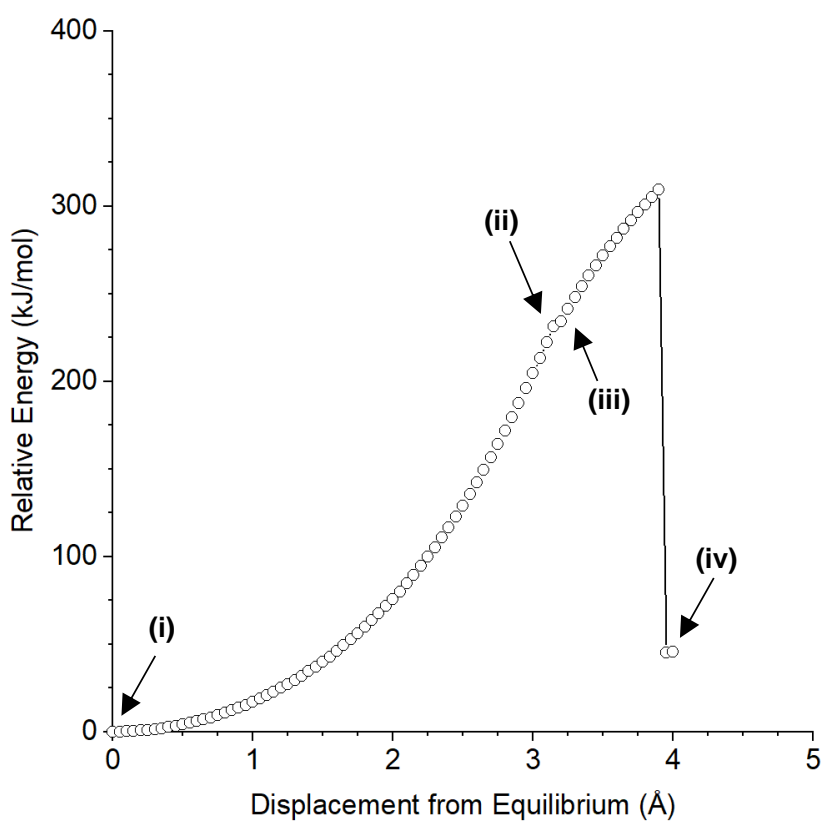

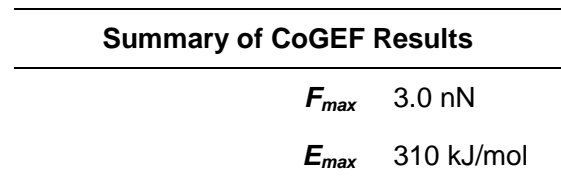

Force-Bond Angle $\quad 0.0^{\circ}$ (i) Equilibrium Geometry

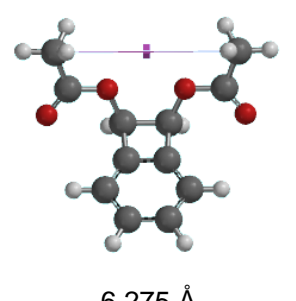

(ii) Immediately Prior to Bond Cleavage

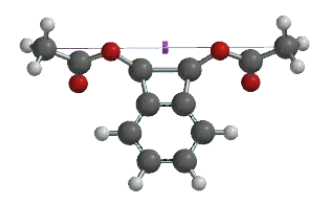

$9.425 \AA$

(iii) Immediately After Bond Cleavage

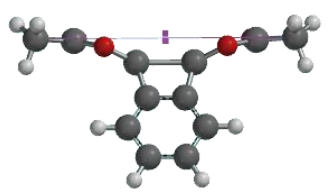

$9.475 \AA$

(iv) After Formation of Double Bonds

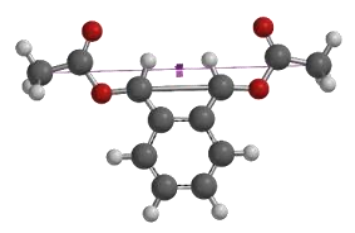

$10.225 \AA$ 

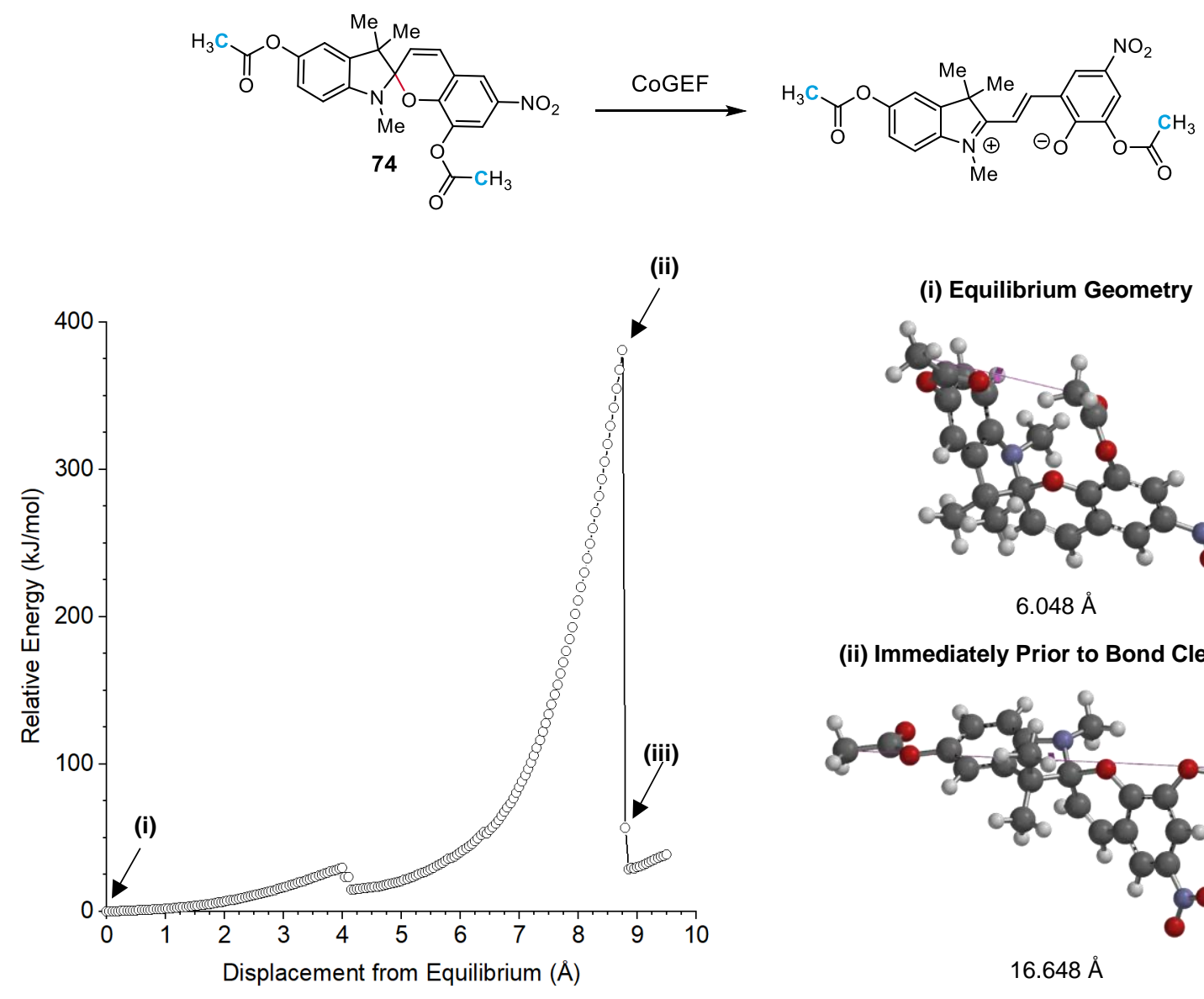

(i) Equilibrium Geometry

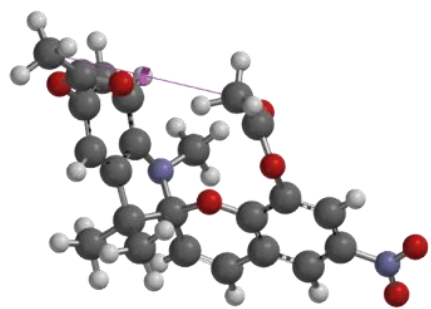

$6.048 \AA$

(ii) Immediately Prior to Bond Cleavage

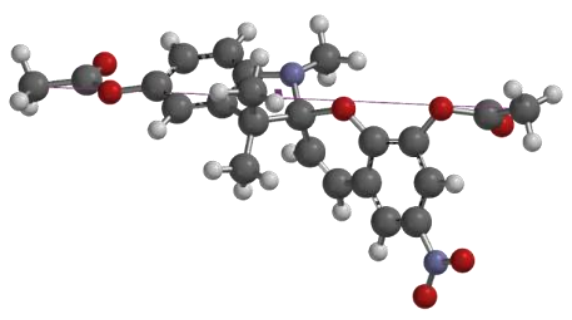

$16.648 \AA$

(iii) Immediately After Bond Cleavage

Summary of CoGEF Results

$$
\begin{array}{ll}
\boldsymbol{F}_{\max } & 4.4 \mathrm{nN} \\
\boldsymbol{E}_{\max } & 381 \mathrm{~kJ} / \mathrm{mol}
\end{array}
$$

Force-Bond Angle $14^{\circ}$

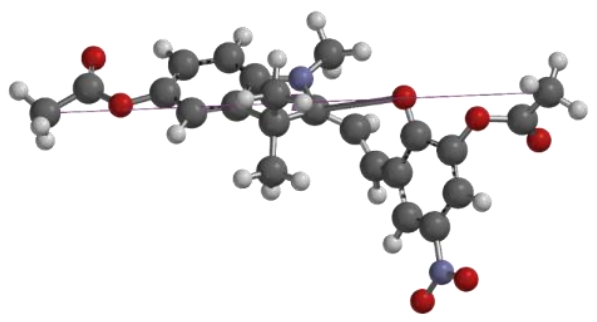

$16.698 \AA$ 

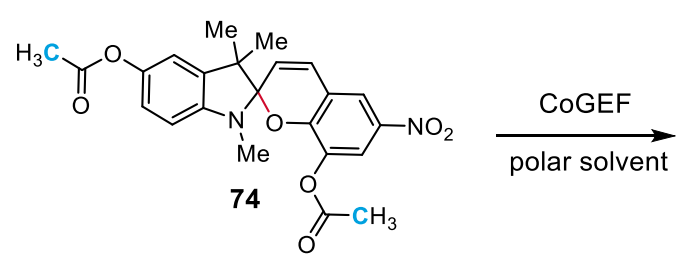

polar solvent $O$

(ii)

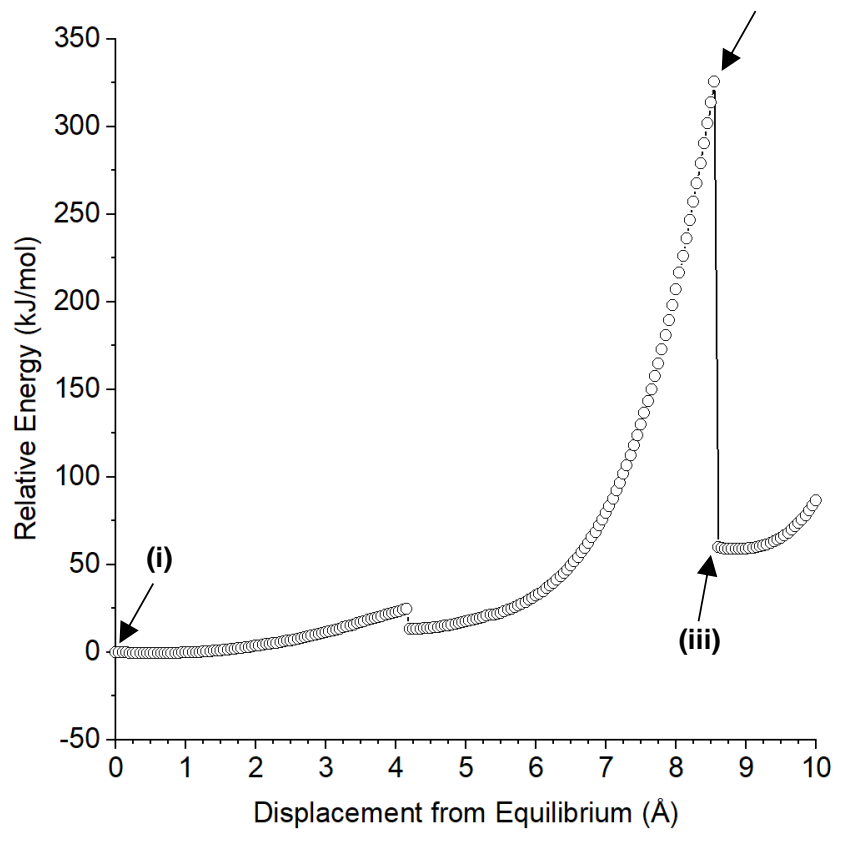

Summary of CoGEF Results

$F_{\max } \quad 4.0 \mathrm{nN}$

$E_{\max } \quad 325 \mathrm{~kJ} / \mathrm{mol}$

Force-Bond Angle $14^{\circ}$<smiles>CC(=O)Oc1ccc2c(c1)C(C)(C)C(/C=C/c1cc([N+](=O)[O-])cc(OC(C)=O)c1O)N2C</smiles>

(i) Equilibrium Geometry

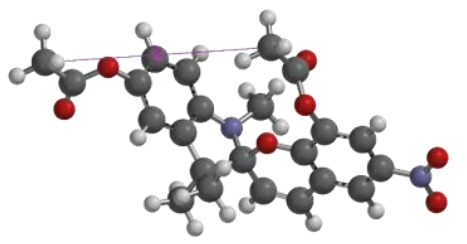

$7.920 \AA$

(ii) Immediately Prior to Bond Cleavage

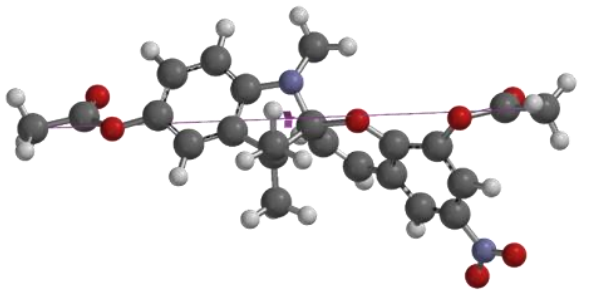

$16.470 \AA$

(iii) Immediately After Bond Cleavage

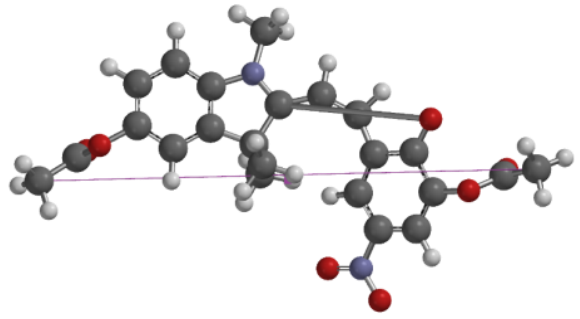

$16.520 \AA$ 


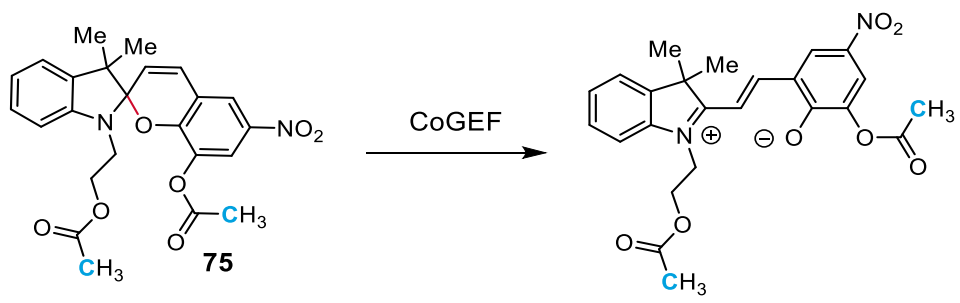

(i) Equilibrium Geometry
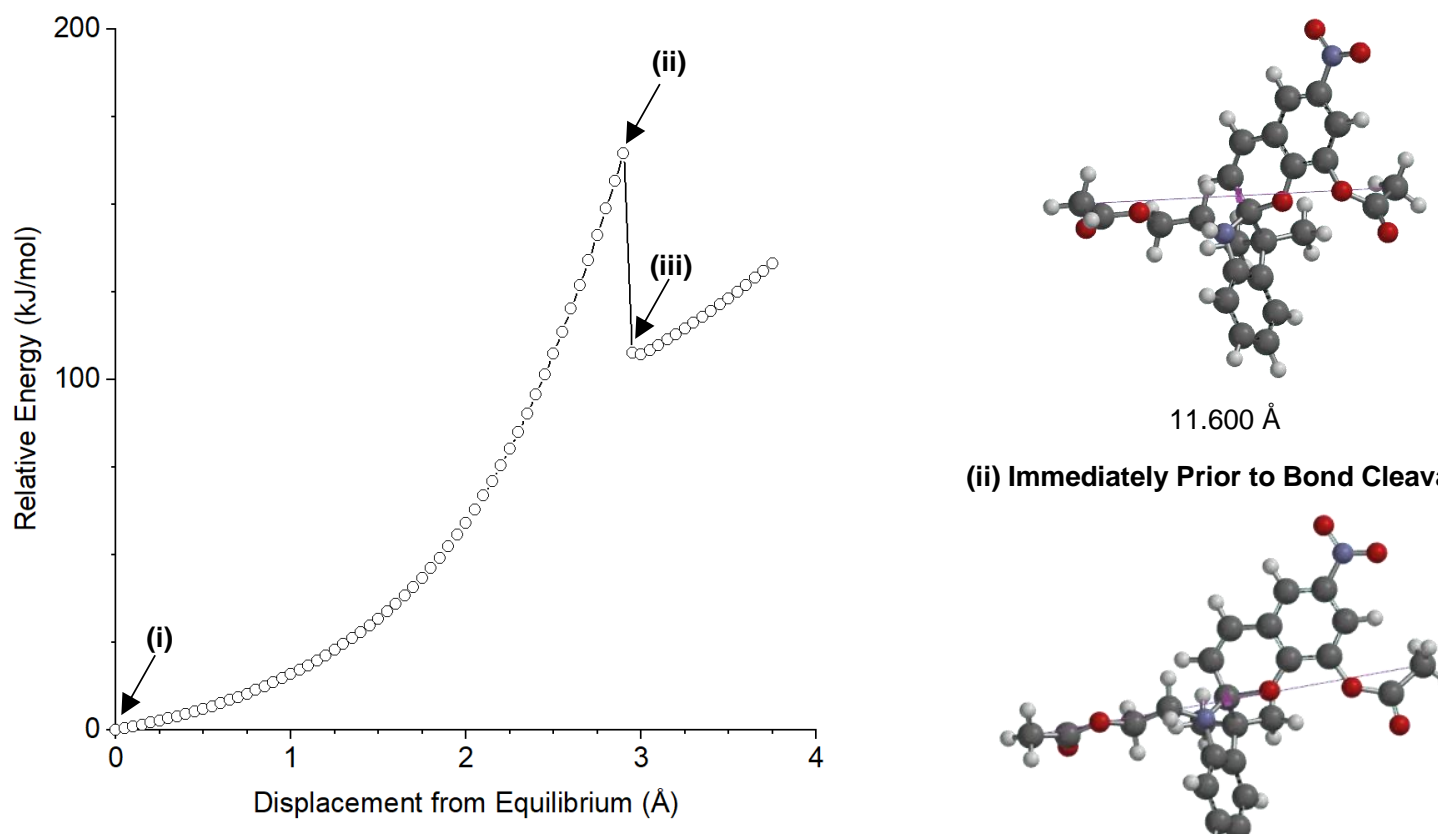

$11.600 \AA$

(ii) Immediately Prior to Bond Cleavage

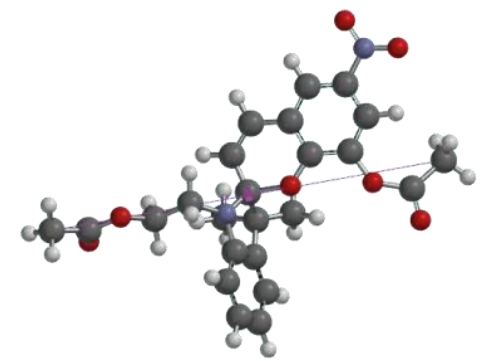

$14.500 \AA$

(iii) Immediately After Bond Cleavage

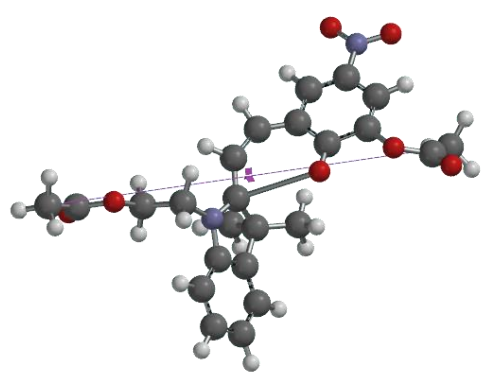

$14.550 \AA$ 

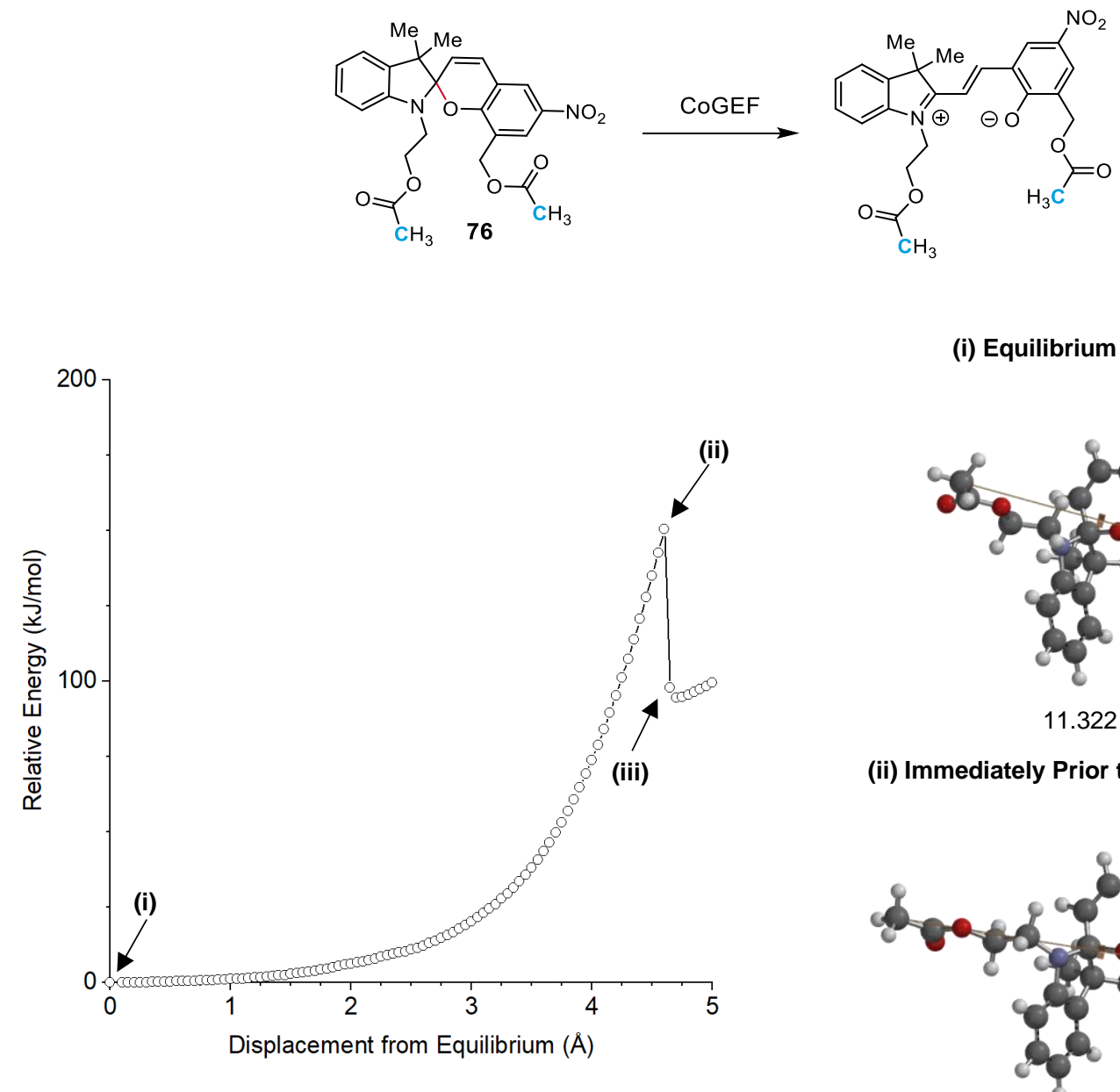

(i) Equilibrium Geometry

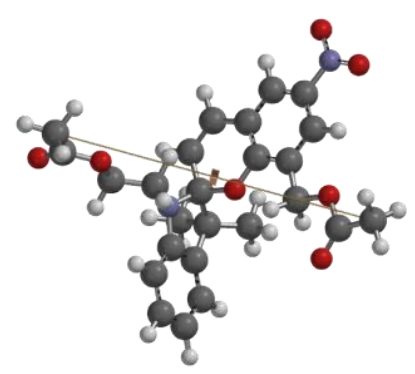

$11.322 \AA$

(ii) Immediately Prior to Bond Cleavage

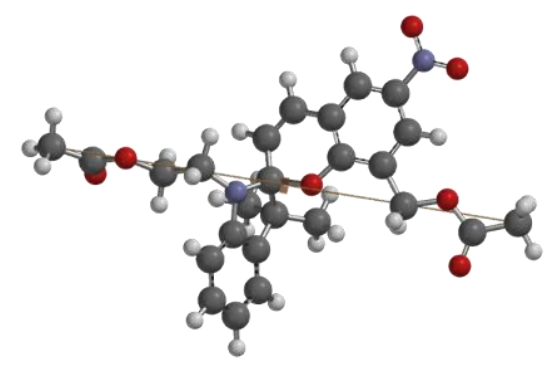

$15.922 \AA$

(iii) Immediately After Bond Cleavage

$\boldsymbol{F}_{\max } \quad 2.6 \mathrm{nN}$

(ii) Immediately After Bond Cleavage

Force-Bond Angle $27^{\circ}$

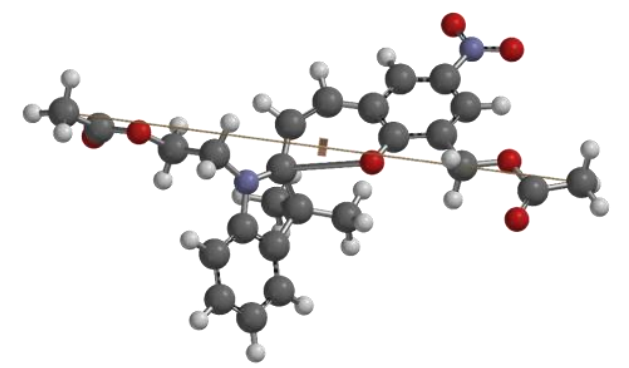

$15.972 \AA$ 

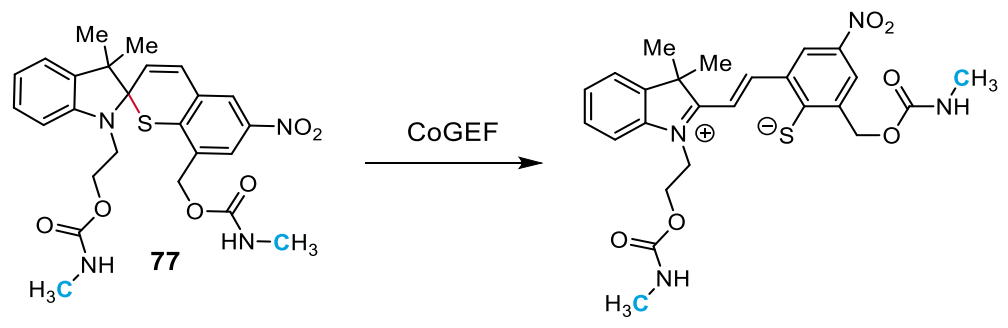

(i) Equilibrium Geometry
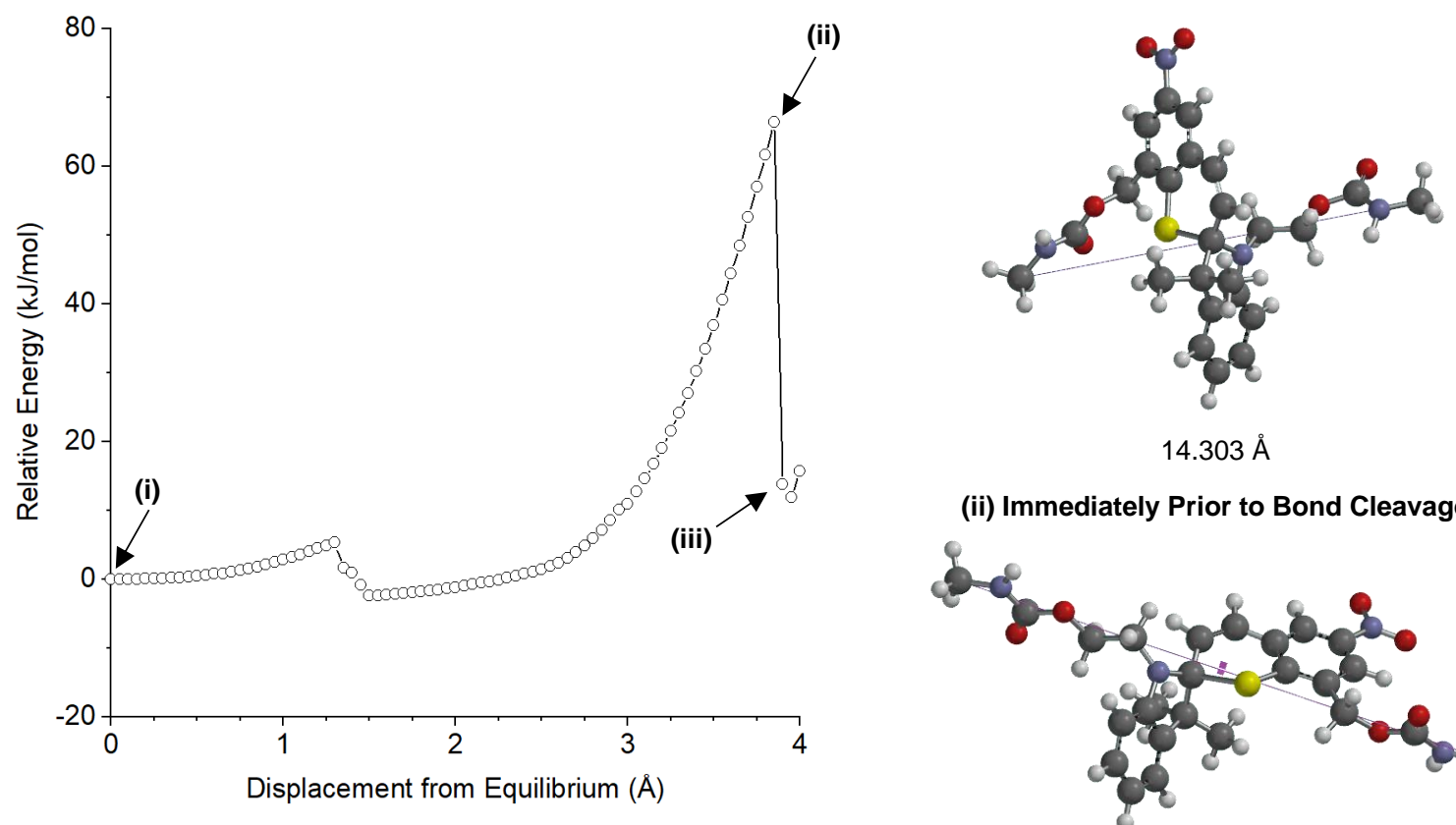

$14.303 \AA$

(ii) Immediately Prior to Bond Cleavage

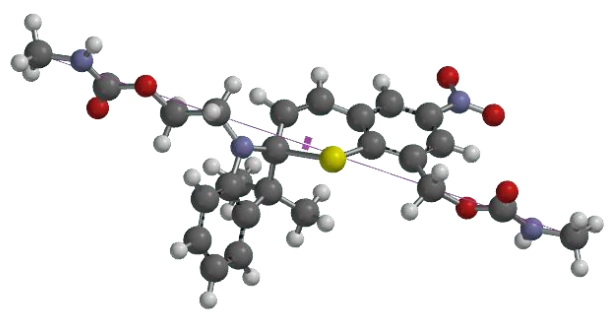

$18.803 \AA$

(iii) Immediately After Bond Cleavage

$$
\begin{array}{ll}
\boldsymbol{F}_{\text {max }} & 2.0 \mathrm{nN} \\
\boldsymbol{E}_{\max } & 74 \mathrm{~kJ} / \mathrm{mol}
\end{array}
$$

Force-Bond Angle $31^{\circ}$

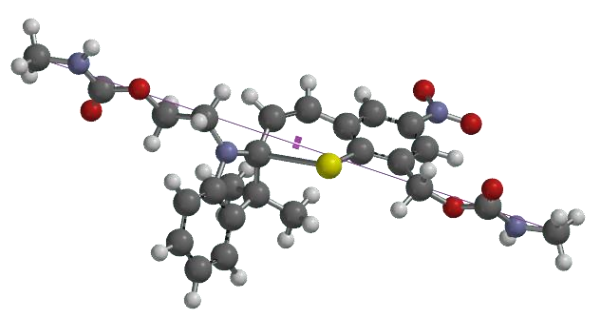

$18.853 \AA$ 

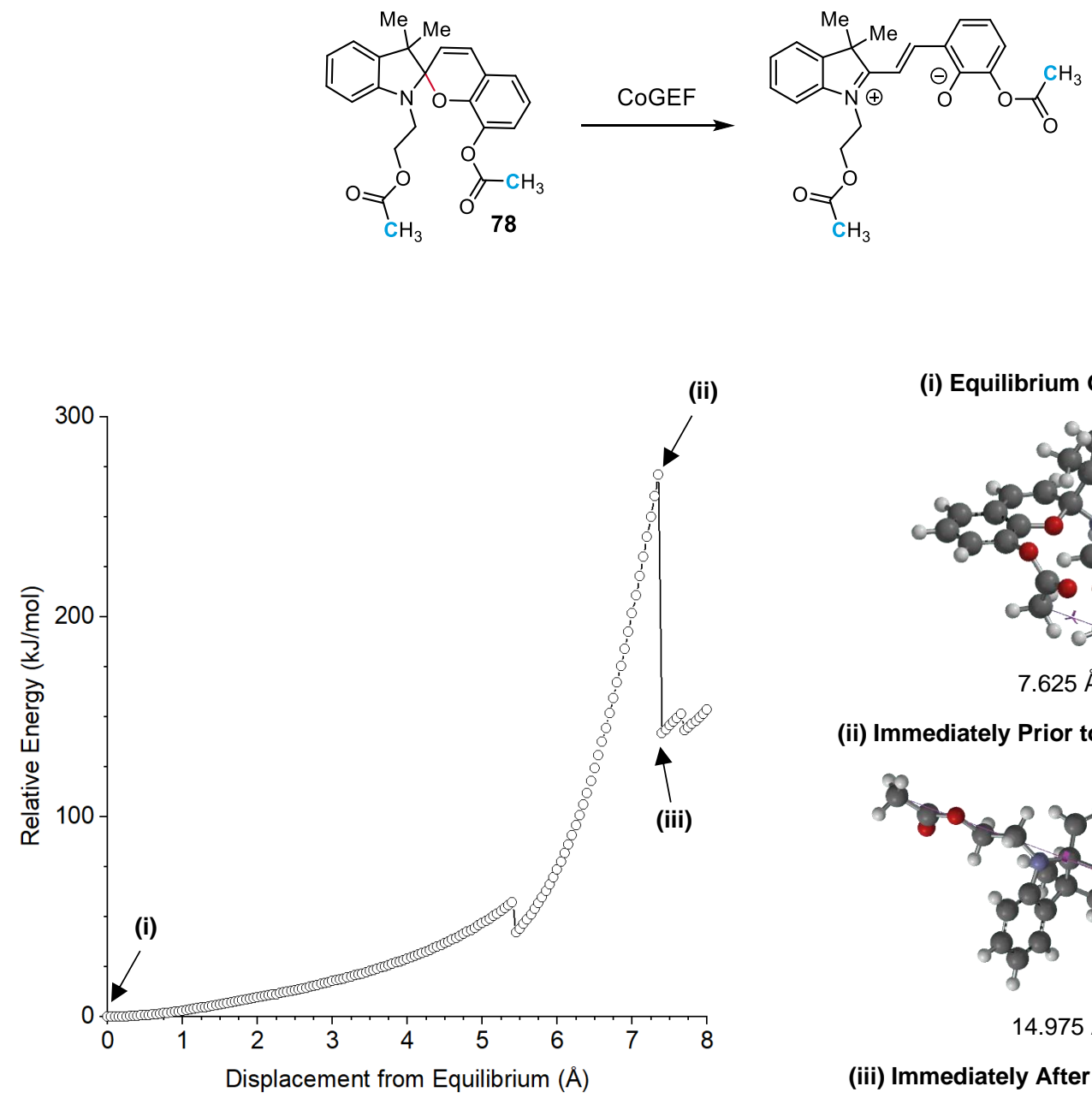

(i) Equilibrium Geometry

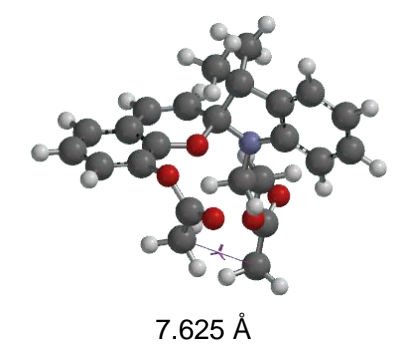

(ii) Immediately Prior to Bond Cleavage

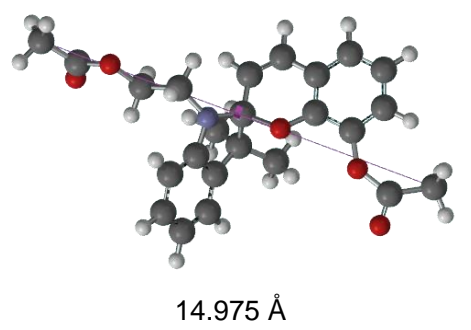

(iii) Immediately After Bond Cleavage

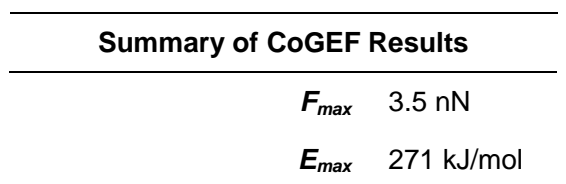

Force-Bond Angle $28^{\circ}$

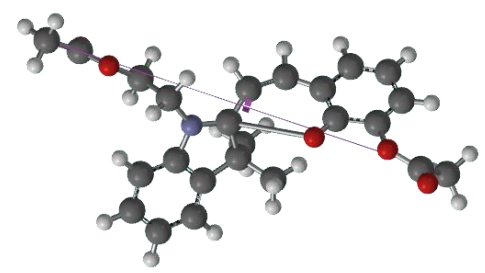

$15.025 \AA$ 


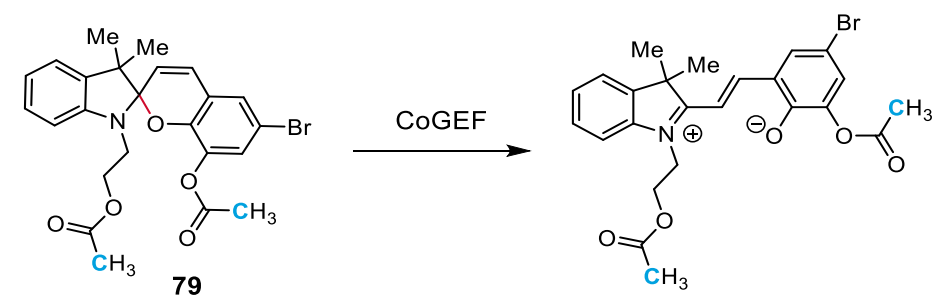

(i) Equilibrium Geometry
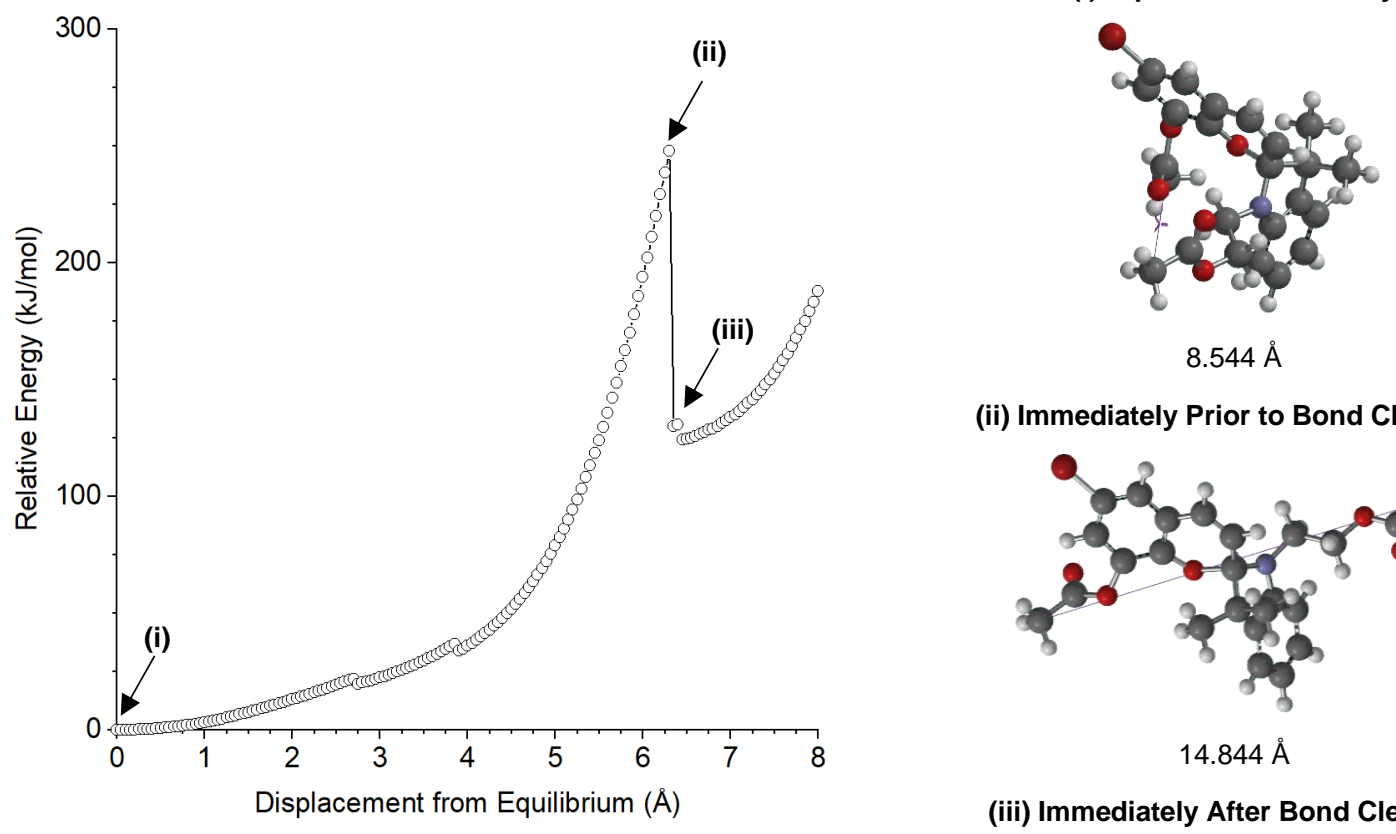

(ii) Immediately Prior to Bond Cleavage

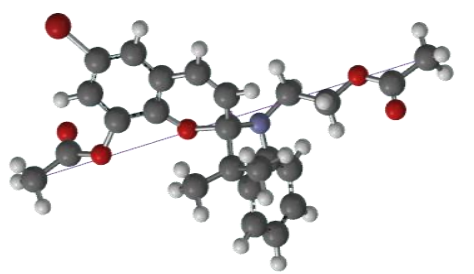

$14.844 \AA$

(iii) Immediately After Bond Cleavage

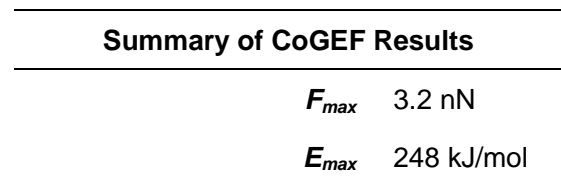

Force-Bond Angle $27^{\circ}$

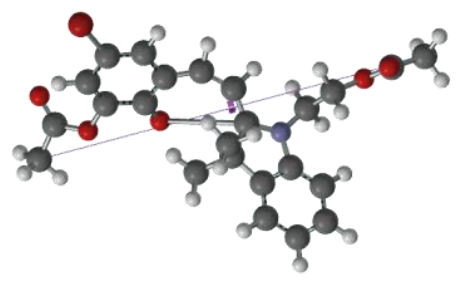

$14.894 \AA$ 

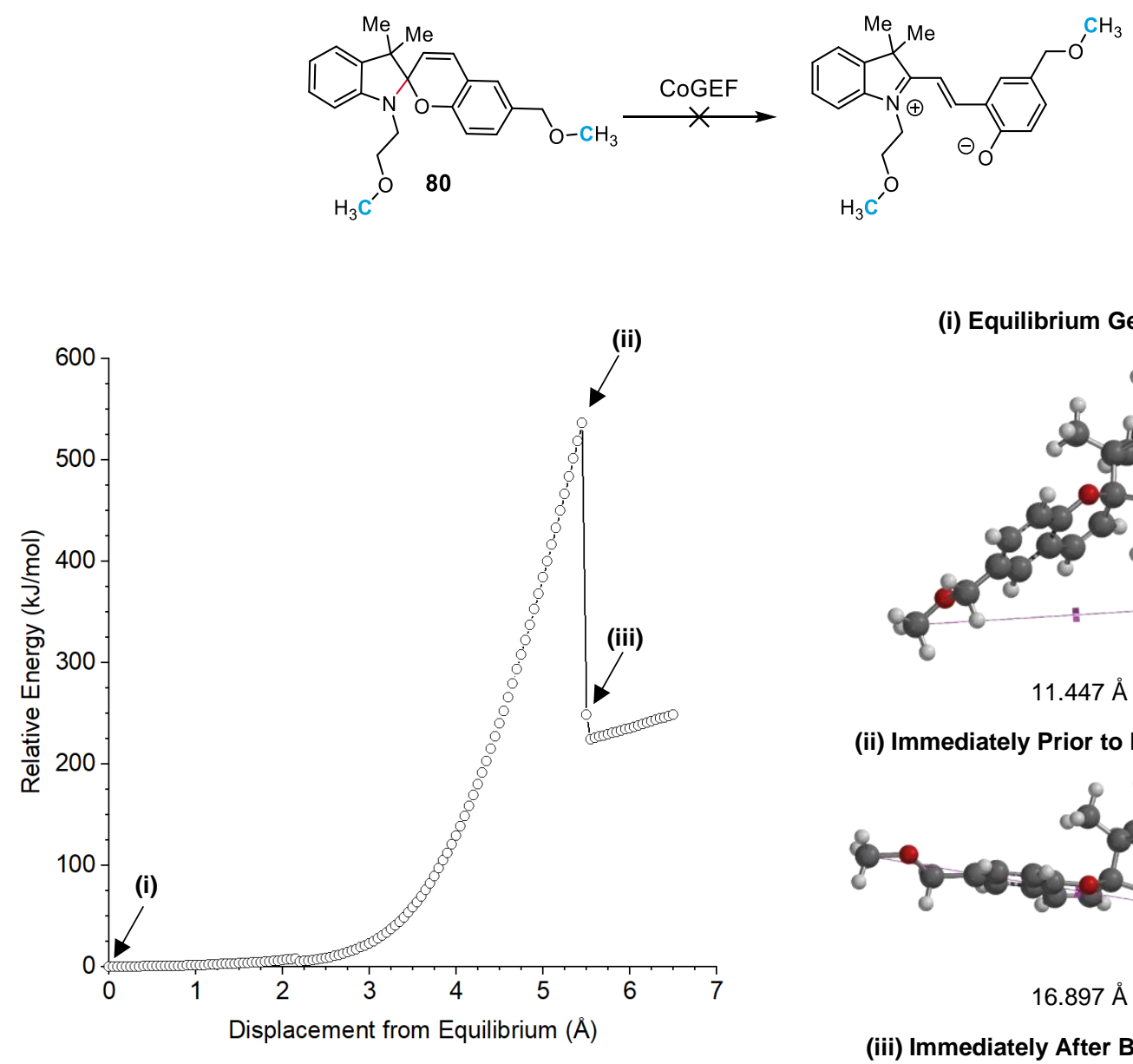

(i) Equilibrium Geometry

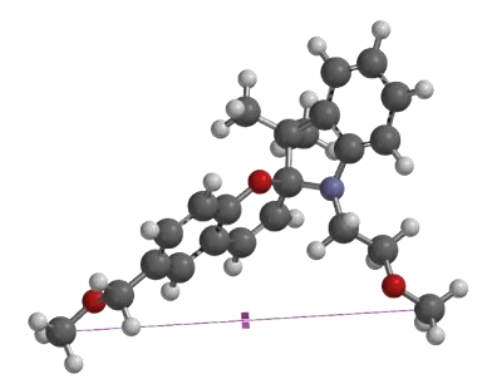

$11.447 \AA$

(ii) Immediately Prior to Bond Cleavage

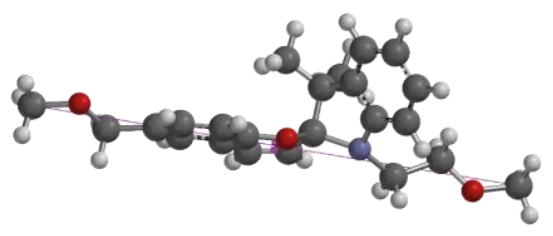

$16.897 \AA$

(iii) Immediately After Bond Cleavage

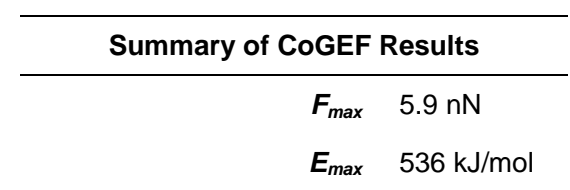

Force-Bond Angle $\quad 47^{\circ}$

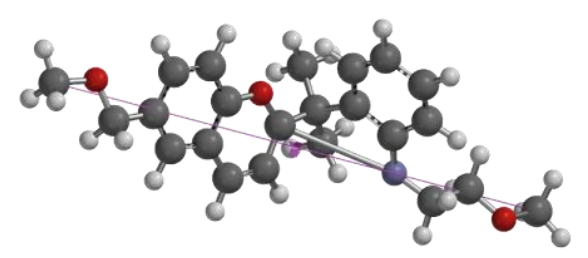

$16.947 \AA$ 

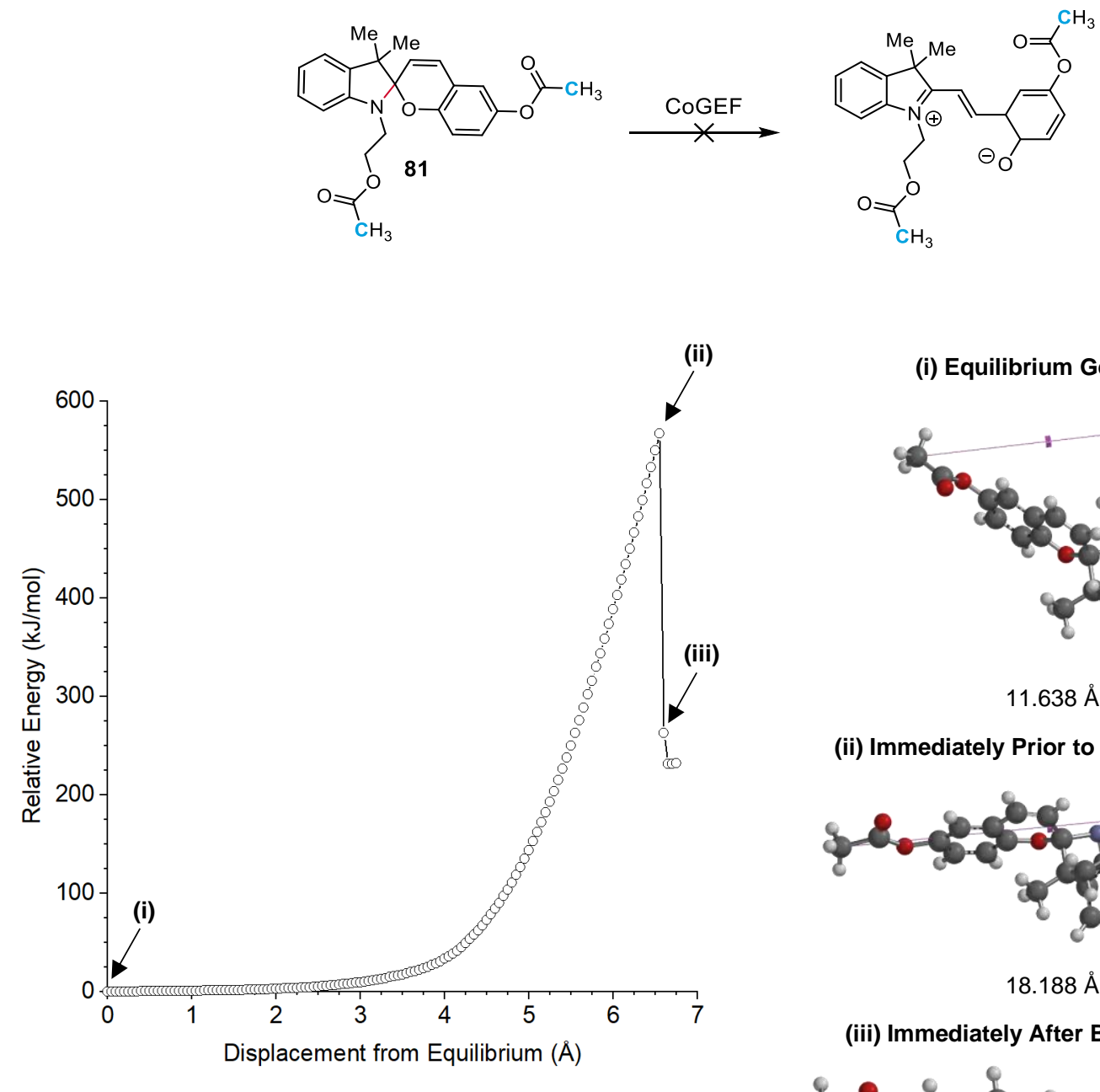

(i) Equilibrium Geometry

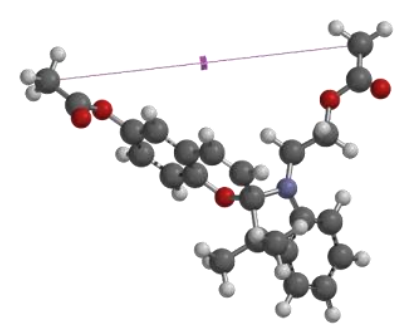

$11.638 \AA$

(ii) Immediately Prior to Bond Cleavage

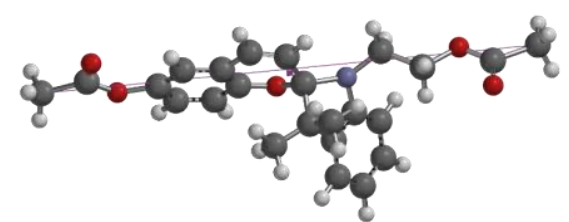

$18.188 \AA$

(iii) Immediately After Bond Cleavage

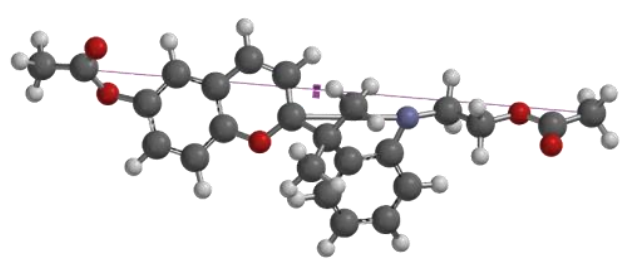

$18.238 \AA$ 

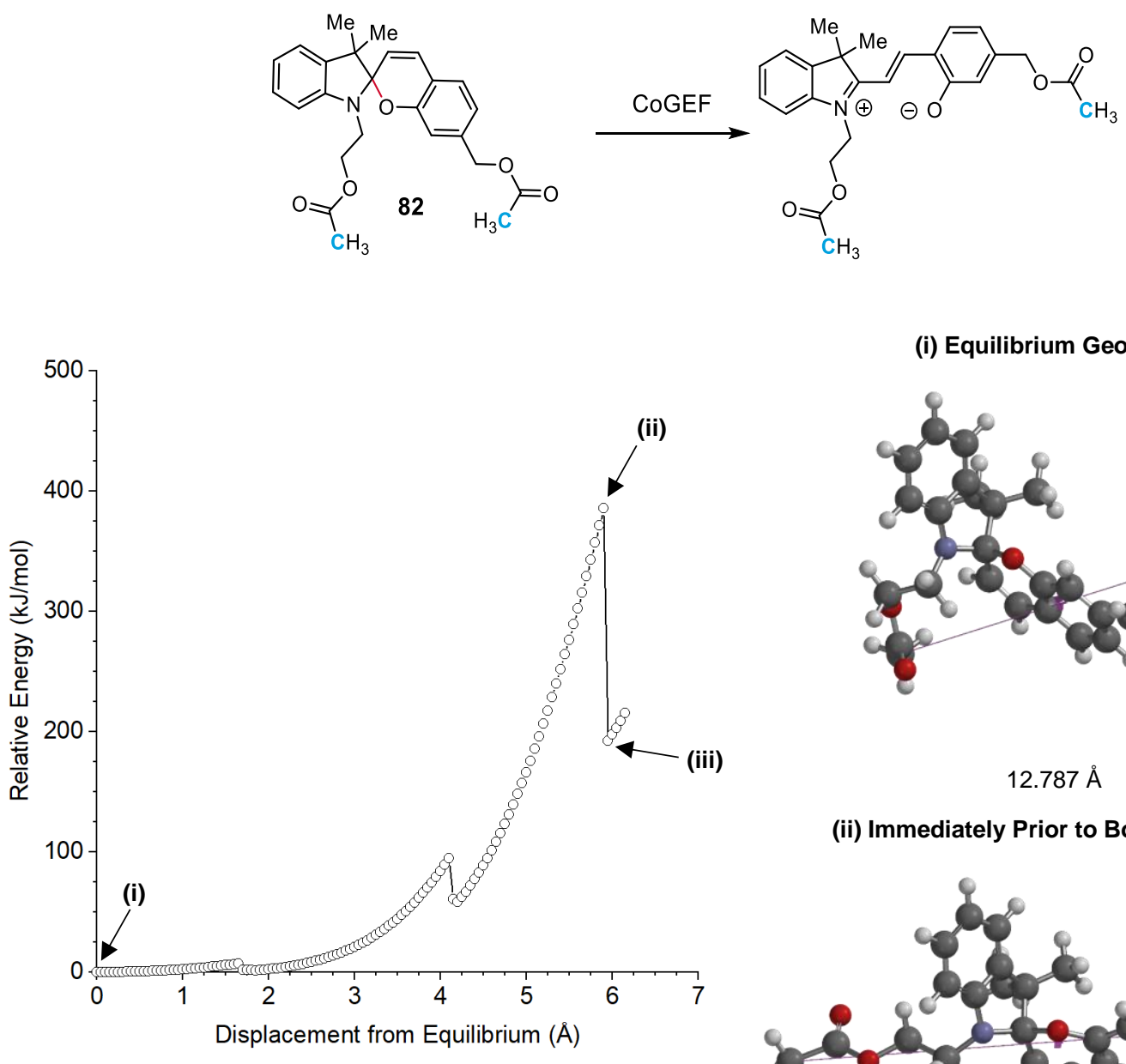

(i) Equilibrium Geometry

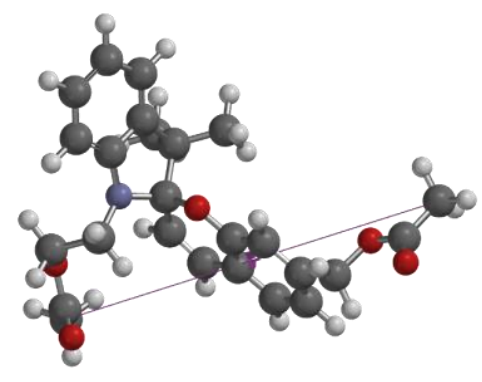

$12.787 \AA$

(ii) Immediately Prior to Bond Cleavage

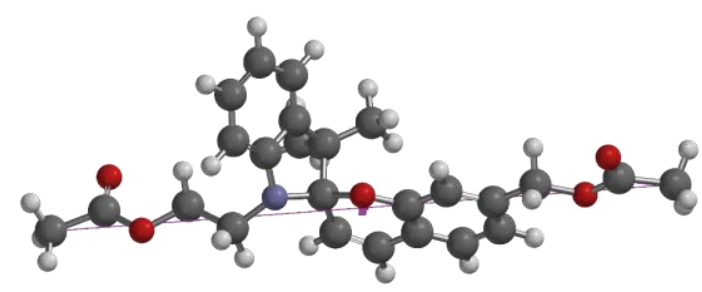

$18.687 \AA$

Summary of CoGEF Results

$$
\begin{array}{ll}
\boldsymbol{F}_{\max } & 4.8 \mathrm{nN} \\
\boldsymbol{E}_{\max } & 386 \mathrm{~kJ} / \mathrm{mol}
\end{array}
$$

Force-Bond Angle $35^{\circ}$

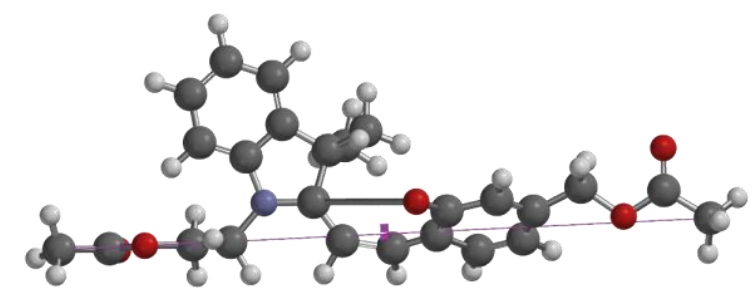

$18.737 \AA$ 

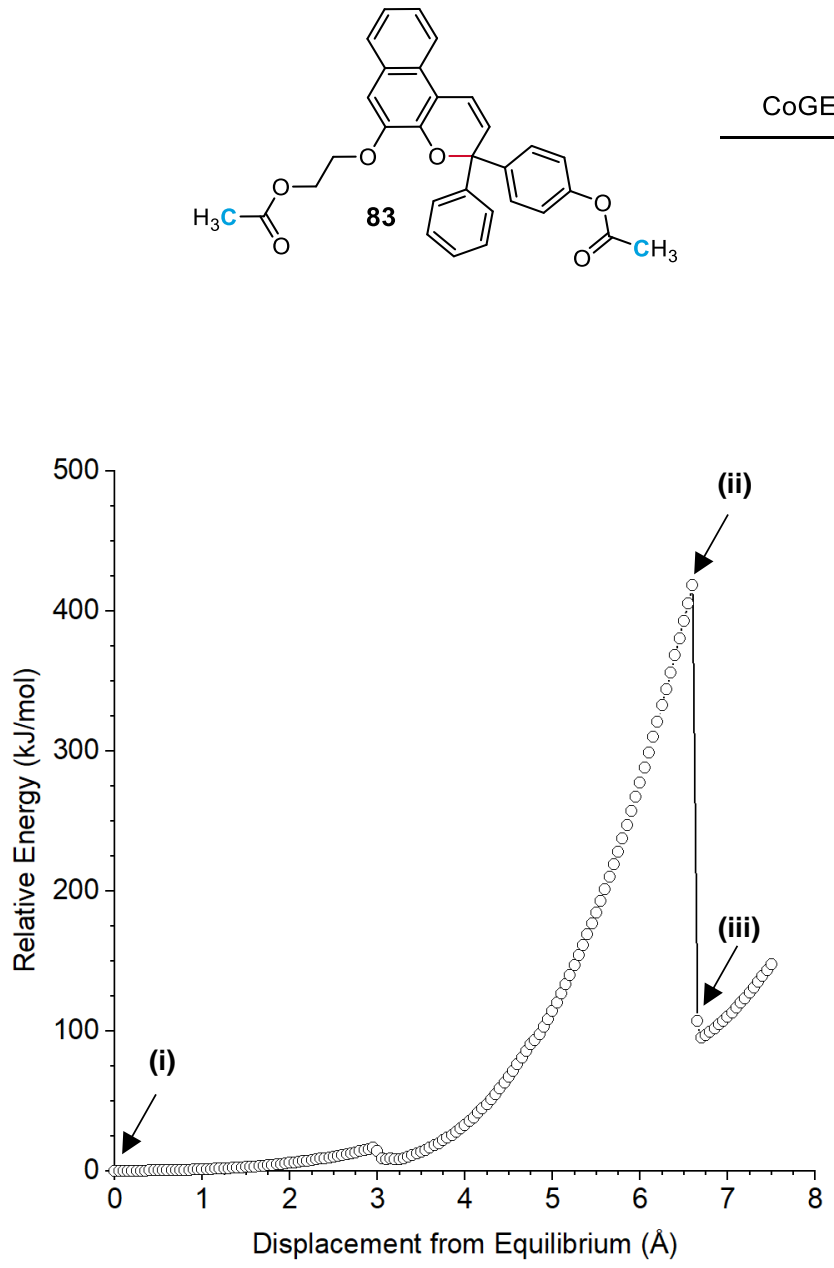

Summary of CoGEF Results

$$
\begin{array}{ll}
\boldsymbol{F}_{\max } & 4.3 \mathrm{nN} \\
\boldsymbol{E}_{\max } & 418 \mathrm{~kJ} / \mathrm{mol}
\end{array}
$$

Force-Bond Angle $29^{\circ}$

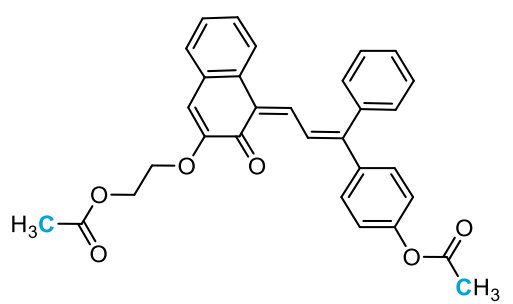

(i) Equilibrium Geometry

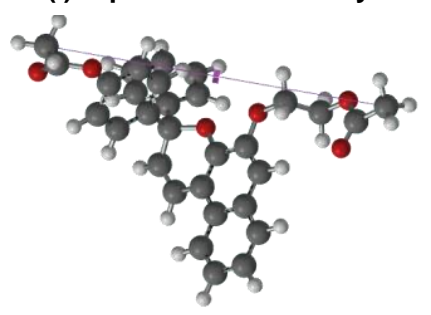

$13.325 \AA$

(ii) Immediately Prior to Bond Cleavage

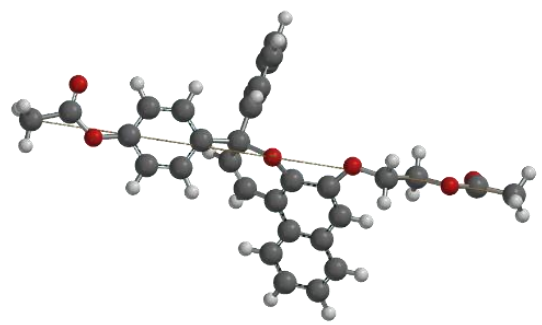

$19.925 \AA$

(iii) Immediately After Bond Cleavage

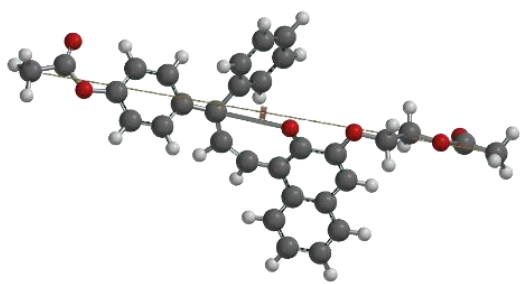

$19.975 \AA$ 


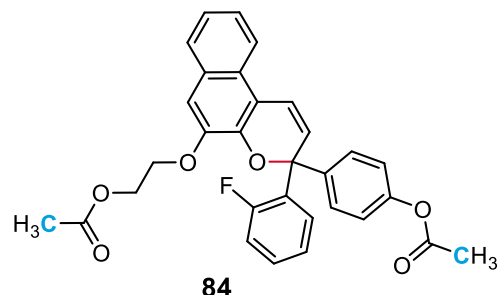

84

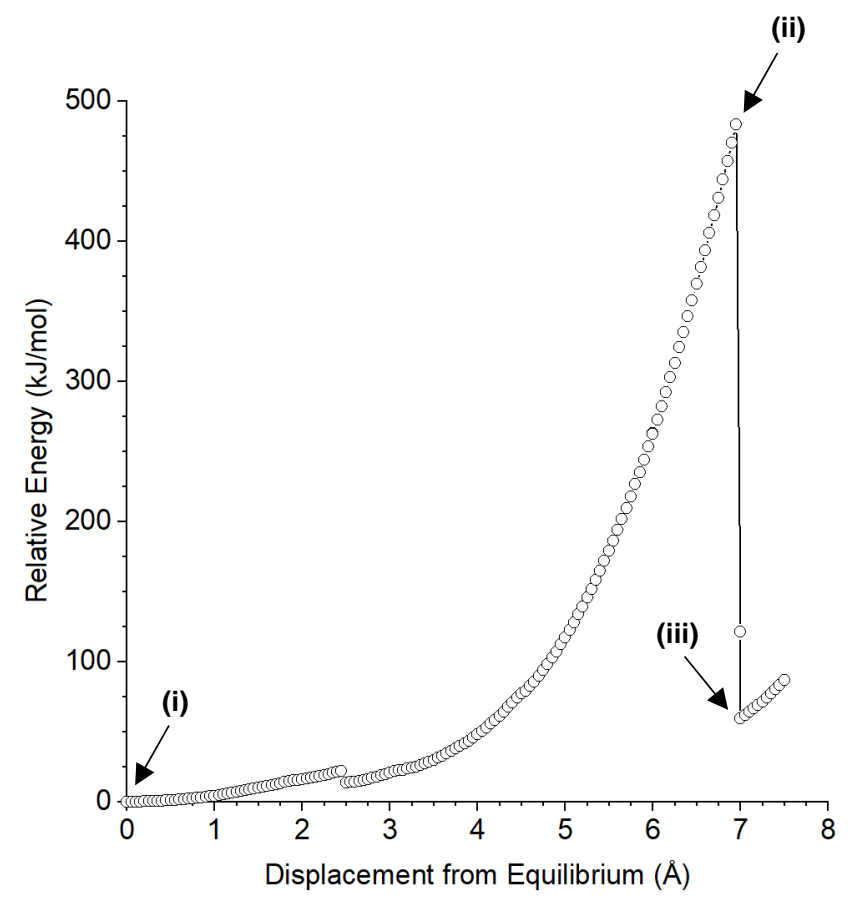

Summary of CoGEF Results

$F_{\max } \quad 4.4 \mathrm{nN}$

$E_{\max } \quad 483 \mathrm{~kJ} / \mathrm{mol}$

Force-Bond Angle $26^{\circ}$

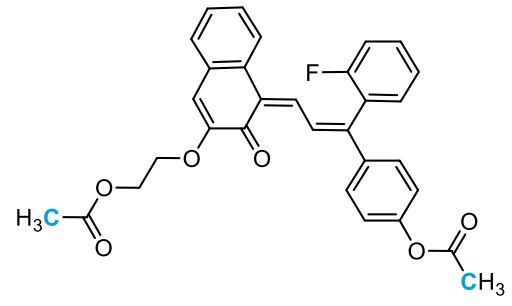

(i) Equilibrium Geometry

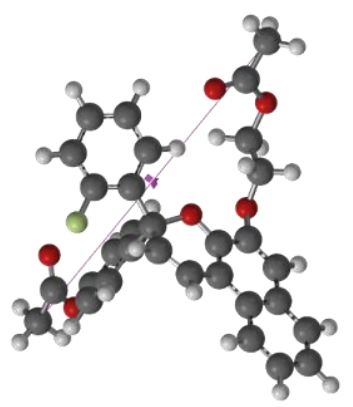

$13.160 \AA$

(ii) Immediately Prior to Bond Cleavage

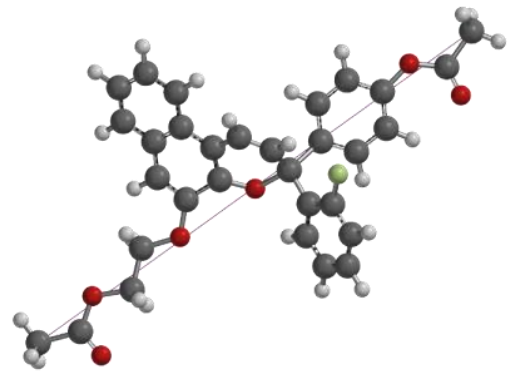

$20.110 \AA$

(iii) Immediately After Bond Cleavage

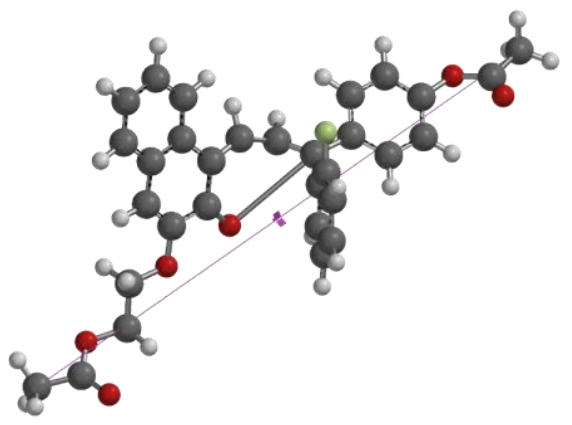

$20.160 \AA$ 

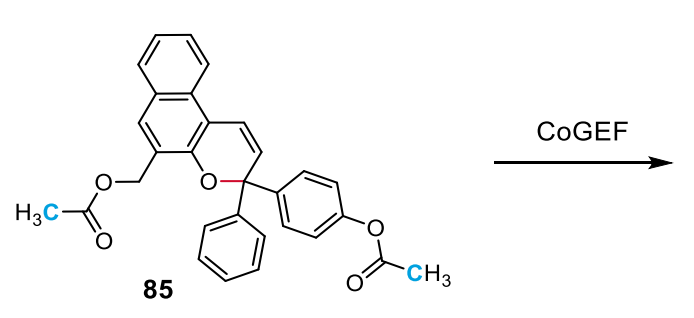

(ii)
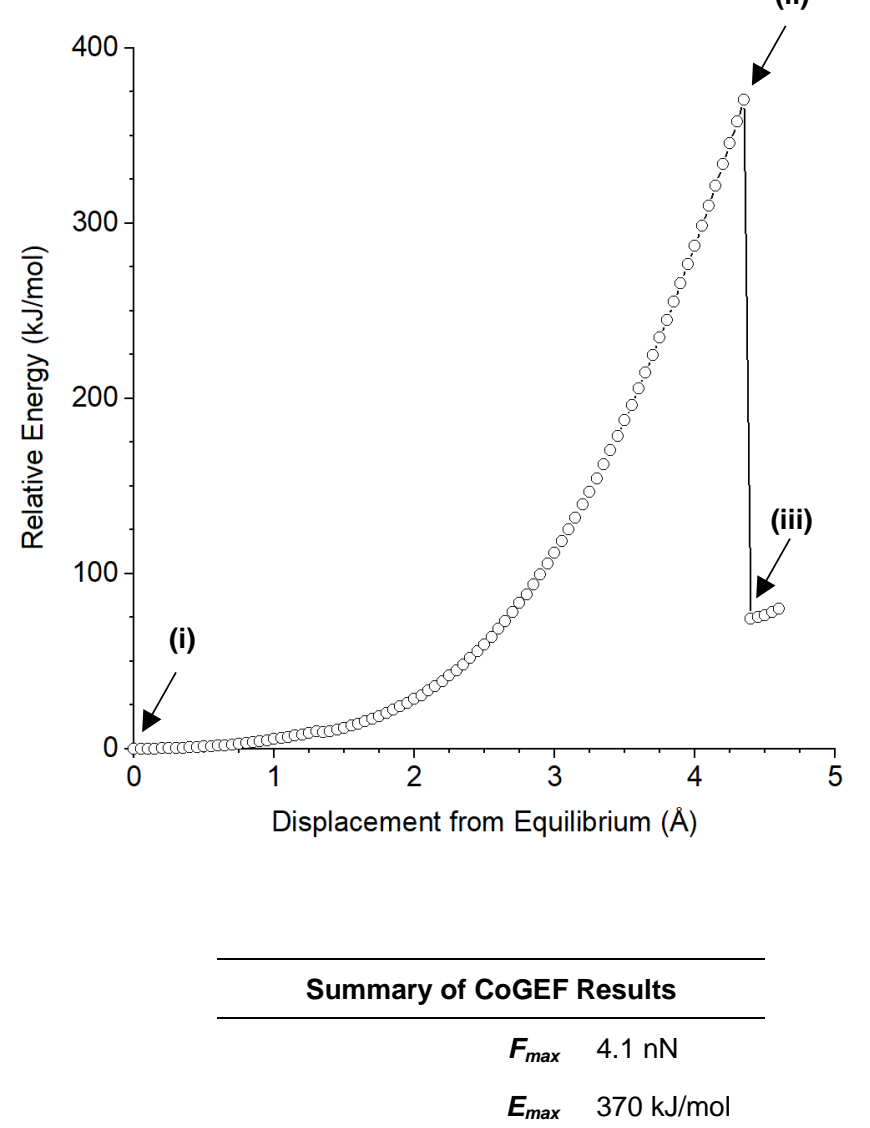

Force-Bond Angle $29^{\circ}$

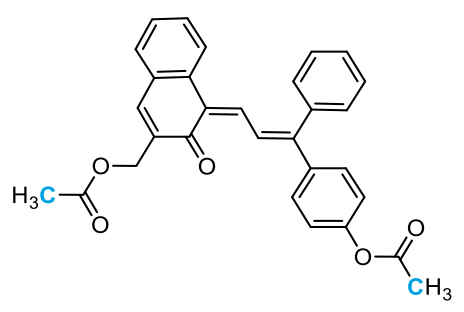

(i) Equilibrium Geometry

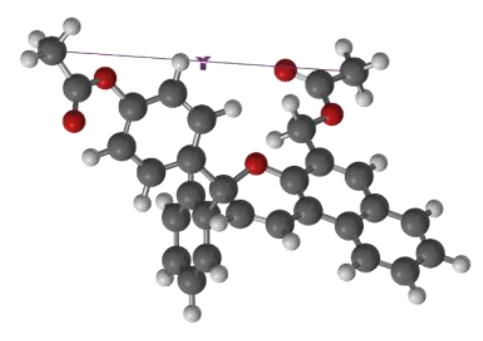

$13.153 \AA$

(ii) Immediately Prior to Bond Cleavage

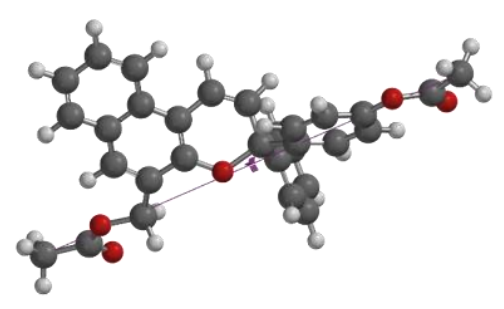

$17.503 \AA$

(iii) Immediately After Bond Cleavage

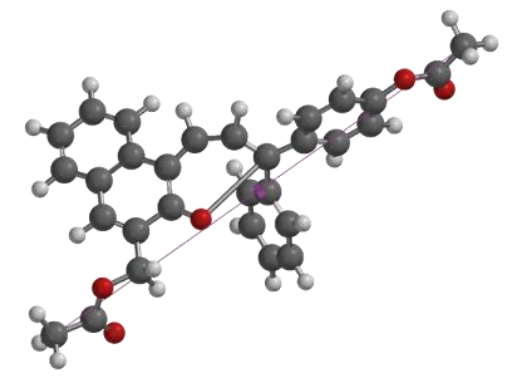

$17.553 \AA$ 

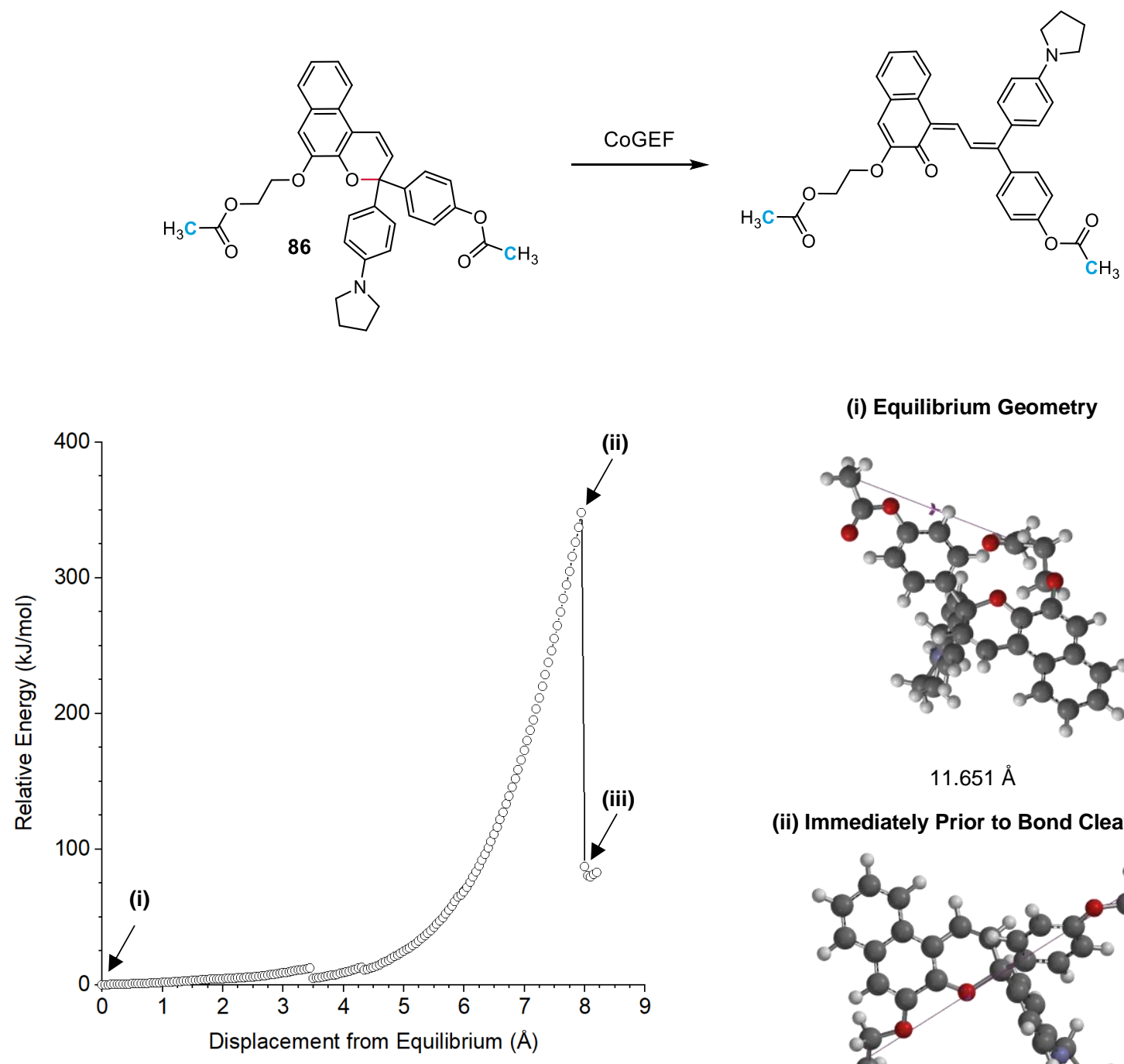

(i) Equilibrium Geometry

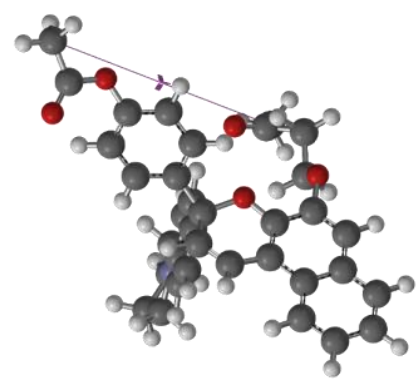

$11.651 \AA$

(ii) Immediately Prior to Bond Cleavage

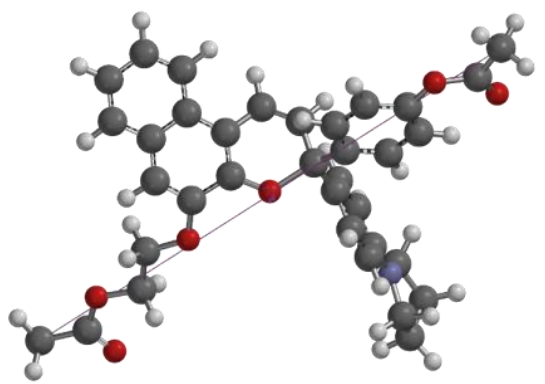

$19.601 \AA$

Summary of CoGEF Results

$F_{\max } \quad 3.7 \mathrm{nN}$

(iii) Immediately After Bond Cleavage

$E_{\max } 348 \mathrm{~kJ} / \mathrm{mol}$

Force-Bond Angle $30^{\circ}$

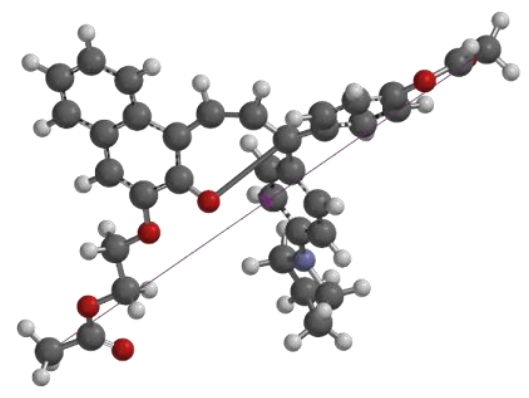

$19.651 \AA$ 


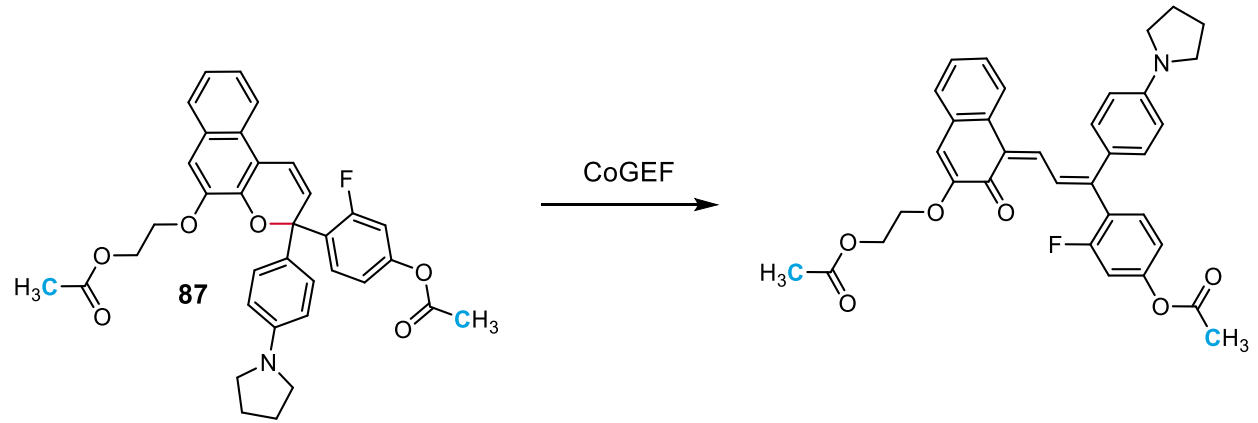

(i) Equilibrium Geometry

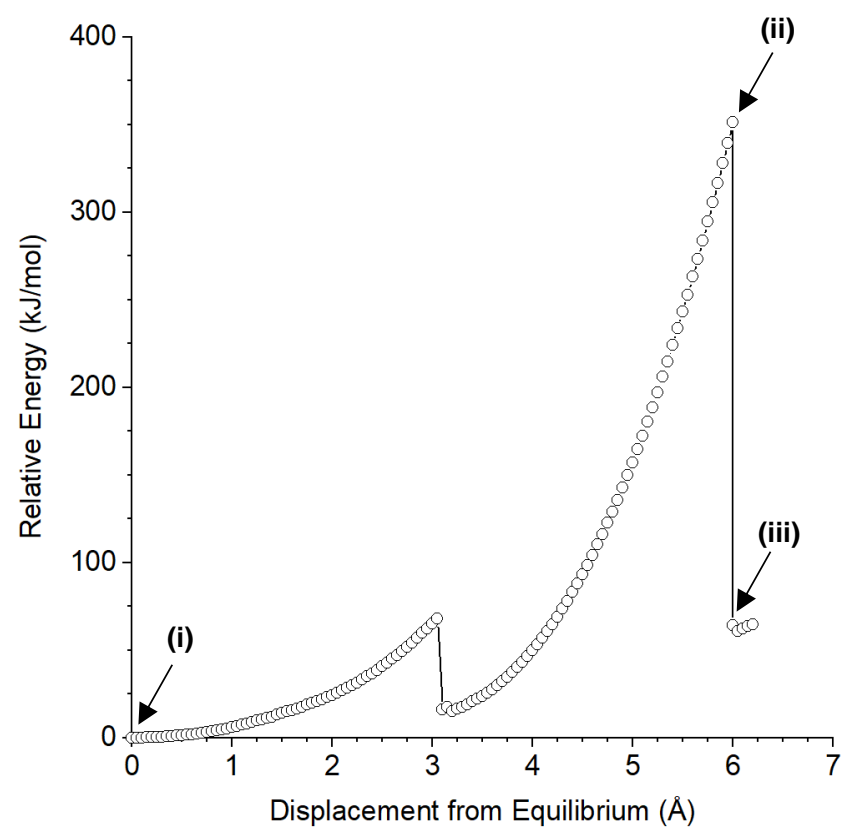

Summary of CoGEF Results

$F_{\max } \quad 3.7 \mathrm{nN}$

$E_{\max } \quad 334 \mathrm{~kJ} / \mathrm{mol}$

Force-Bond Angle $33^{\circ}$

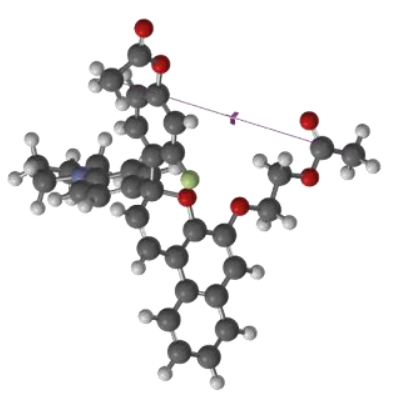

$11.190 \AA$

(ii) Immediately Prior to Bond Cleavage

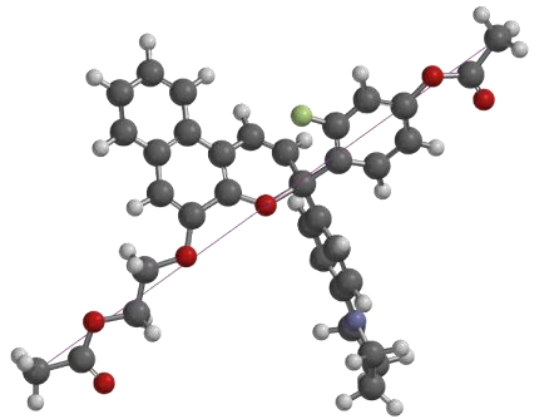

$19.590 \AA$

(iii) Immediately After Bond Cleavage

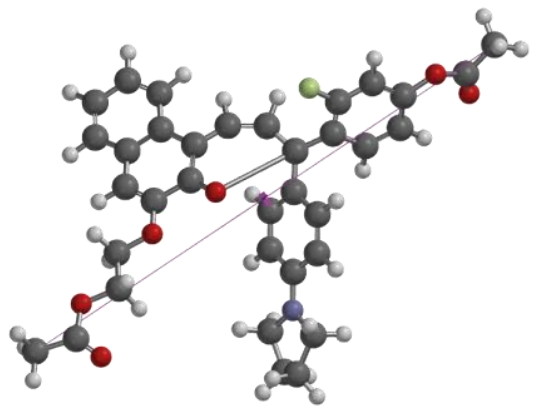

$19.640 \AA$ 


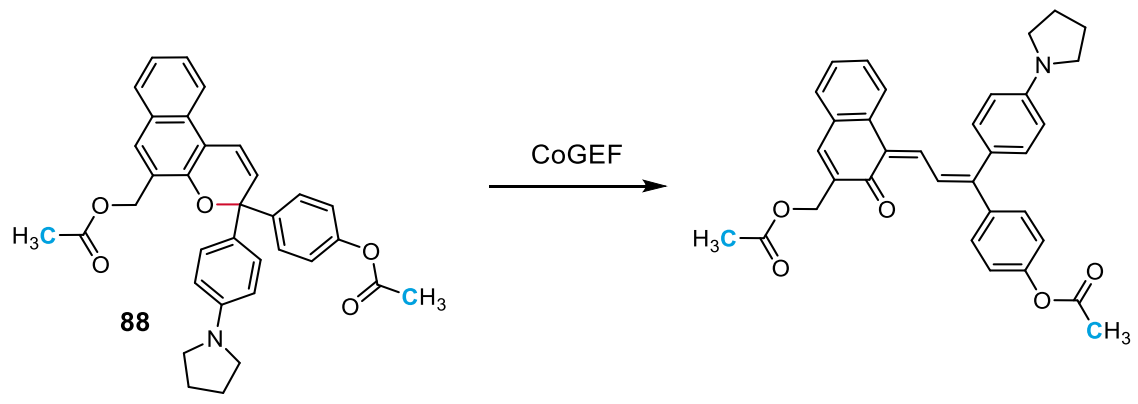

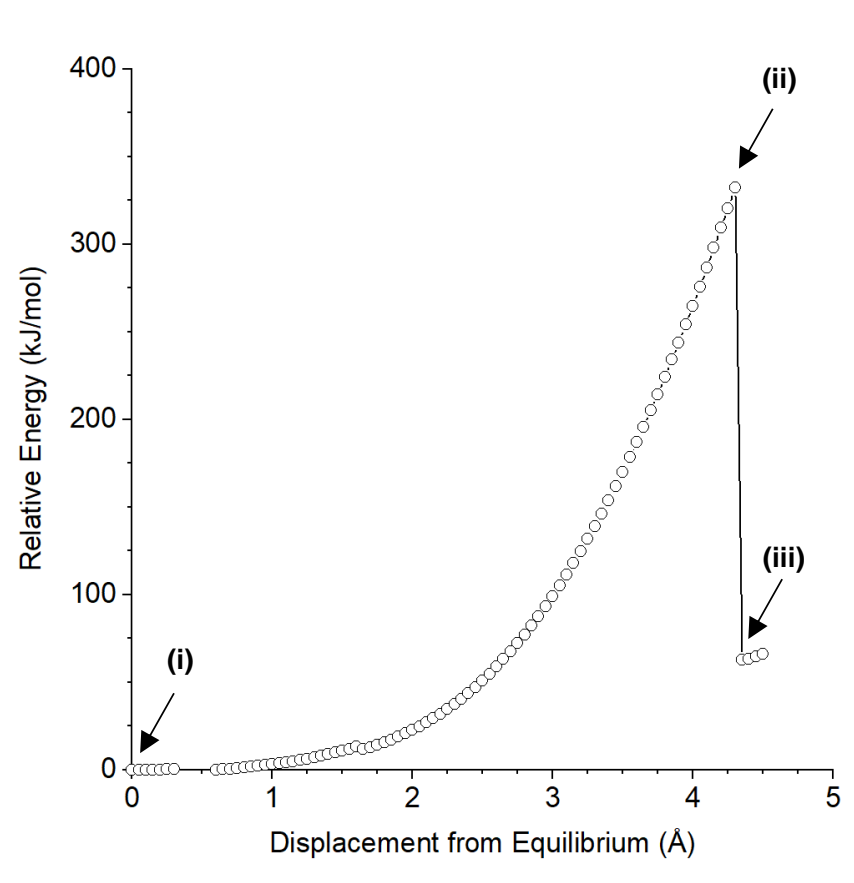

Summary of CoGEF Results

$\boldsymbol{F}_{\max } \quad 3.9 \mathrm{nN}$

$E_{\max } \quad 332 \mathrm{~kJ} / \mathrm{mol}$

Force-Bond Angle $30^{\circ}$ (i) Equilibrium Geometry

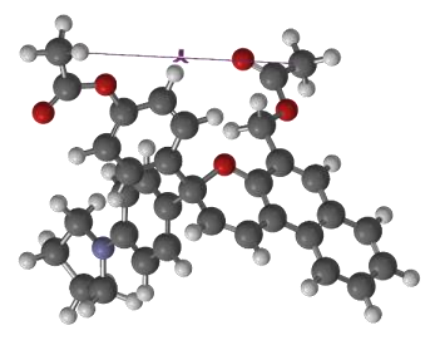

$13.054 \AA$

(ii) Immediately Prior to Bond Cleavage

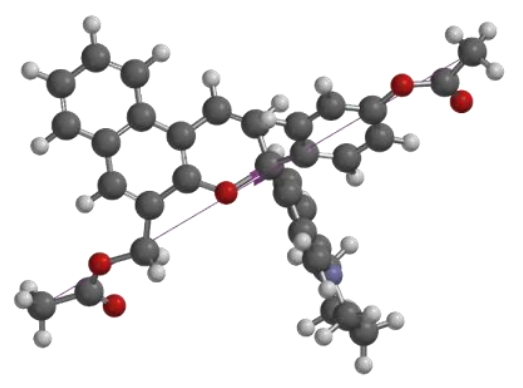

$17.354 \AA$

(iii) Immediately After Bond Cleavage

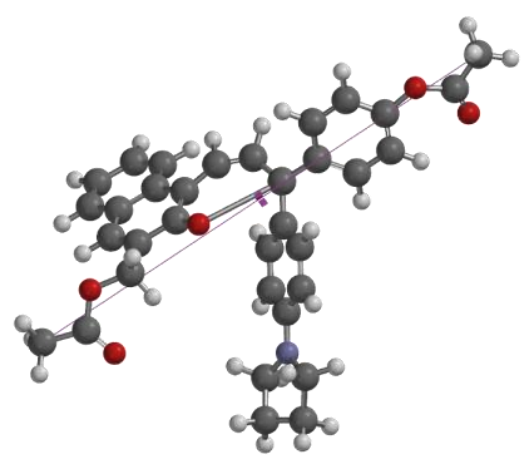

$17.404 \AA$ 


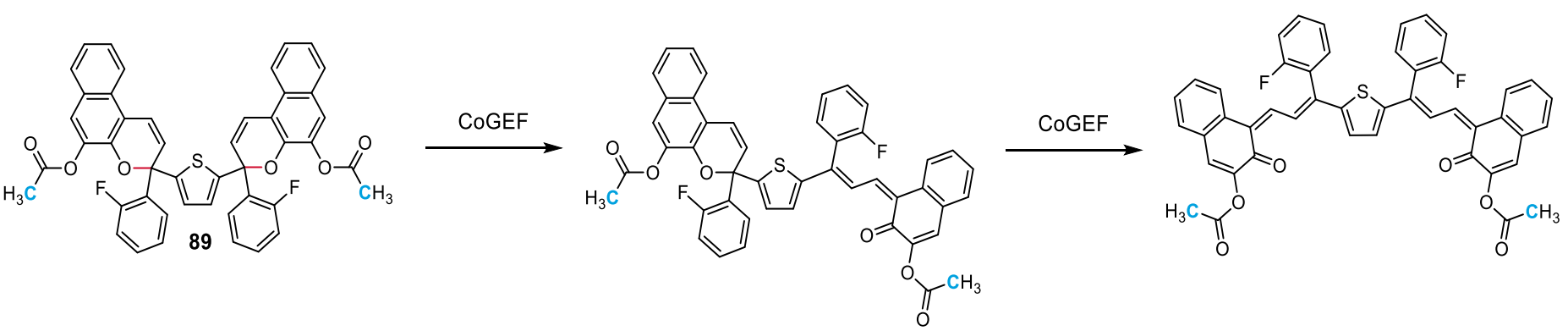
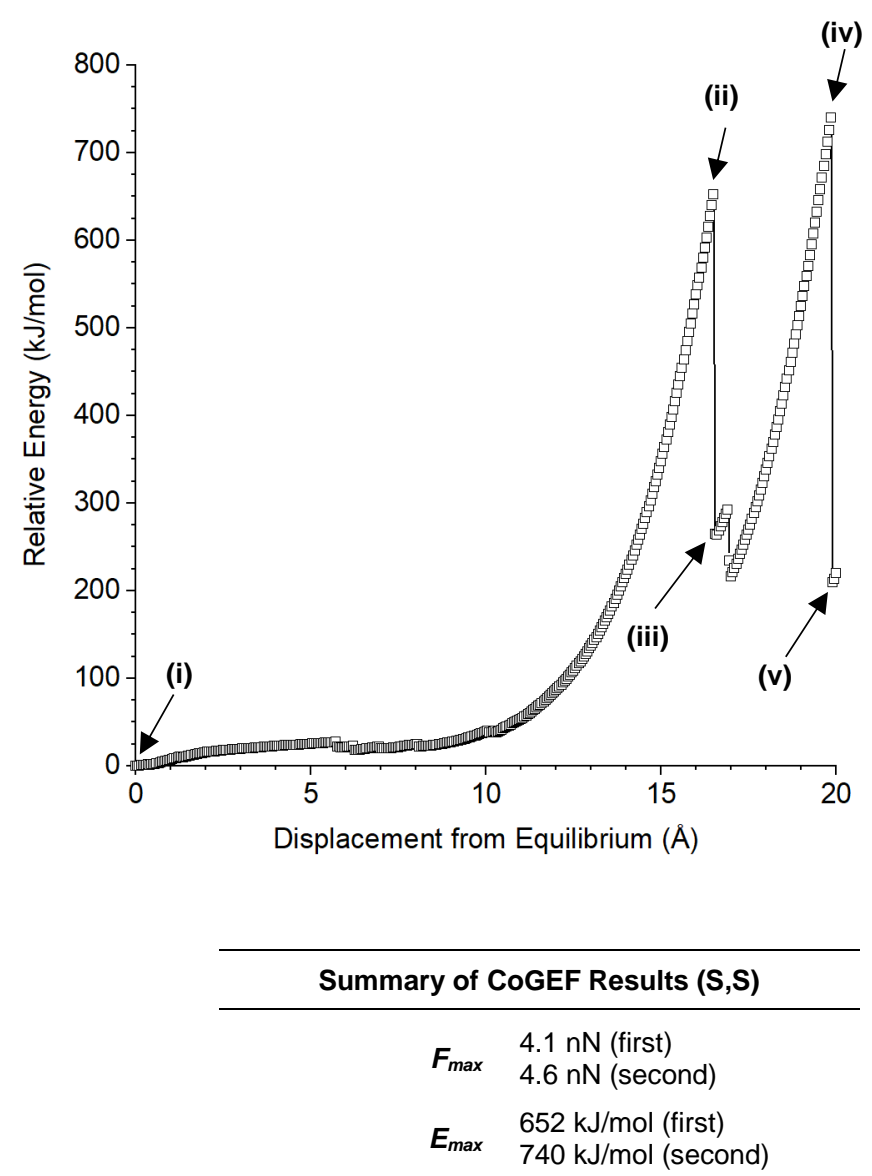

(iv)

(i) Equilibrium Geometry

Force-Bond Angle $25^{\circ}$ (first), $27^{\circ}$ (second)

The results presented here correspond to the (S,S)-isomer of compound 82. The CoGEF results for the (R,S)-isomer are similar:

\begin{tabular}{|c|c|}
\hline \multicolumn{2}{|c|}{ Summary of CoGEF Results $(R, S)$} \\
\hline$F_{\max }$ & $\begin{array}{l}4.1 \mathrm{nN} \text { (first) } \\
4.5 \mathrm{nN} \text { (second) }\end{array}$ \\
\hline$E_{\max }$ & $\begin{array}{l}644 \mathrm{~kJ} / \mathrm{mol} \text { (first) } \\
727 \mathrm{~kJ} / \mathrm{mol} \text { (second) }\end{array}$ \\
\hline Force-Bond Angle & $24^{\circ}$ (first), $26^{\circ}$ (second) \\
\hline
\end{tabular}

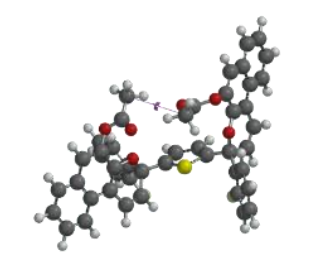

$4.159 \AA$

(ii) Immediately Prior to First Bond Cleavage

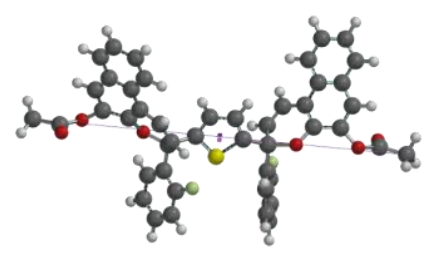

$20.659 \AA$

(iii) Immediately After Bond First Cleavage

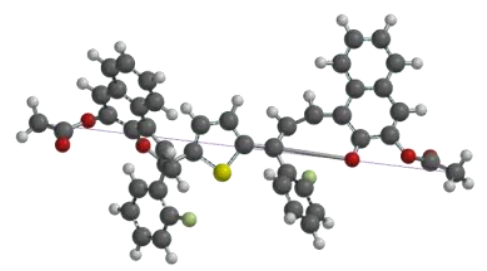

$20.709 \AA$

(iv) Immediately Prior to Second Bond Cleavage

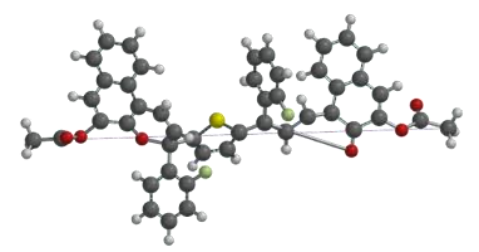

$24.009 \AA$

(v) Immediately After Bond Second Cleavage

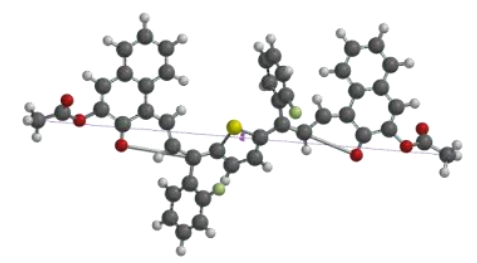

$24.059 \AA$ 

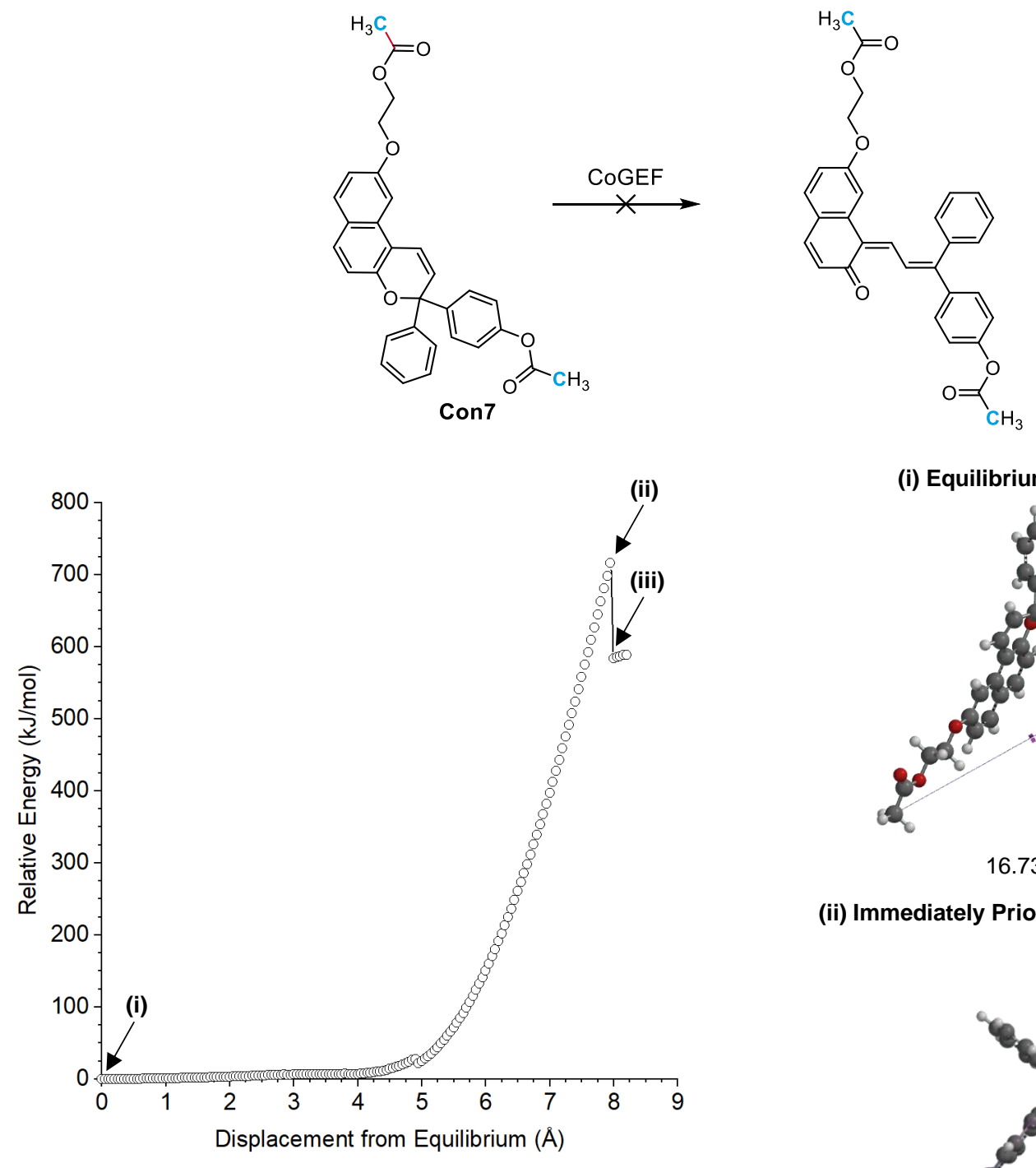

(i) Equilibrium Geometry

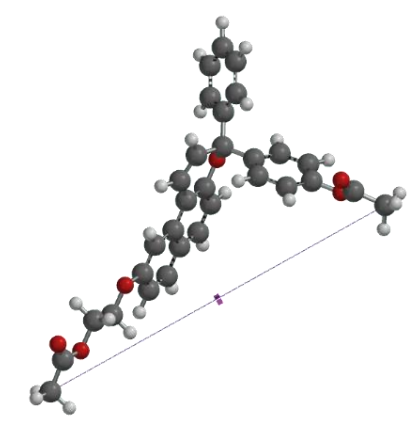

$16.735 \AA$

(ii) Immediately Prior to Bond Cleavage

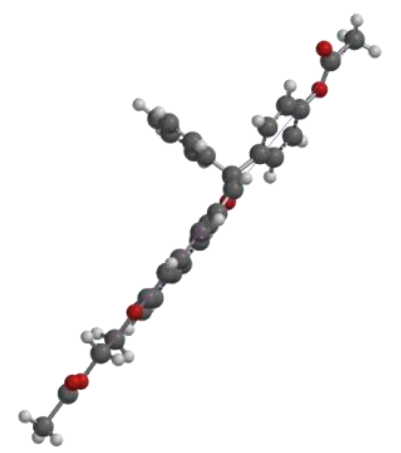

$24.685 \AA$

Summary of CoGEF Results

$\boldsymbol{F}_{\max } \quad 6.0 \mathrm{nN}$

$E_{\max } 716 \mathrm{~kJ} / \mathrm{mol}$

Force-Bond Angle $63^{\circ}$

(iii) Immediately After Bond Cleavage

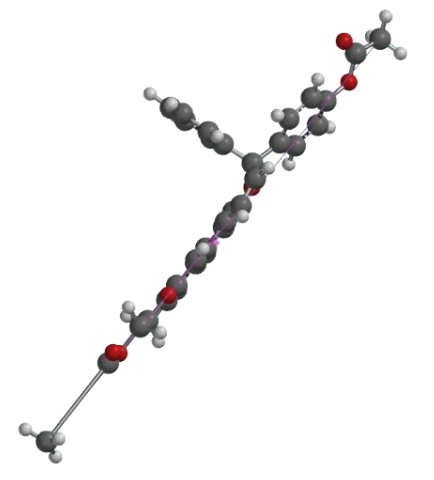

$24.735 \AA$ 


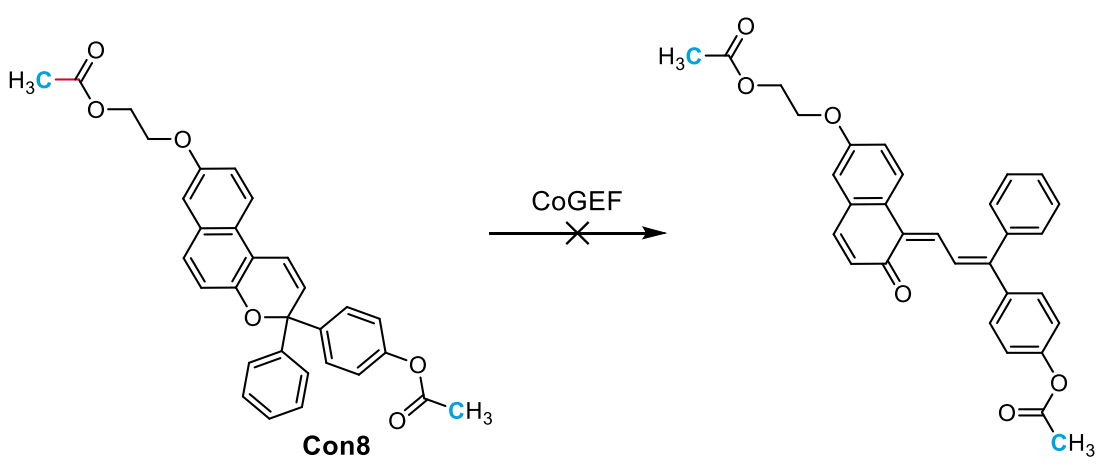

(i) Equilibrium Geometry
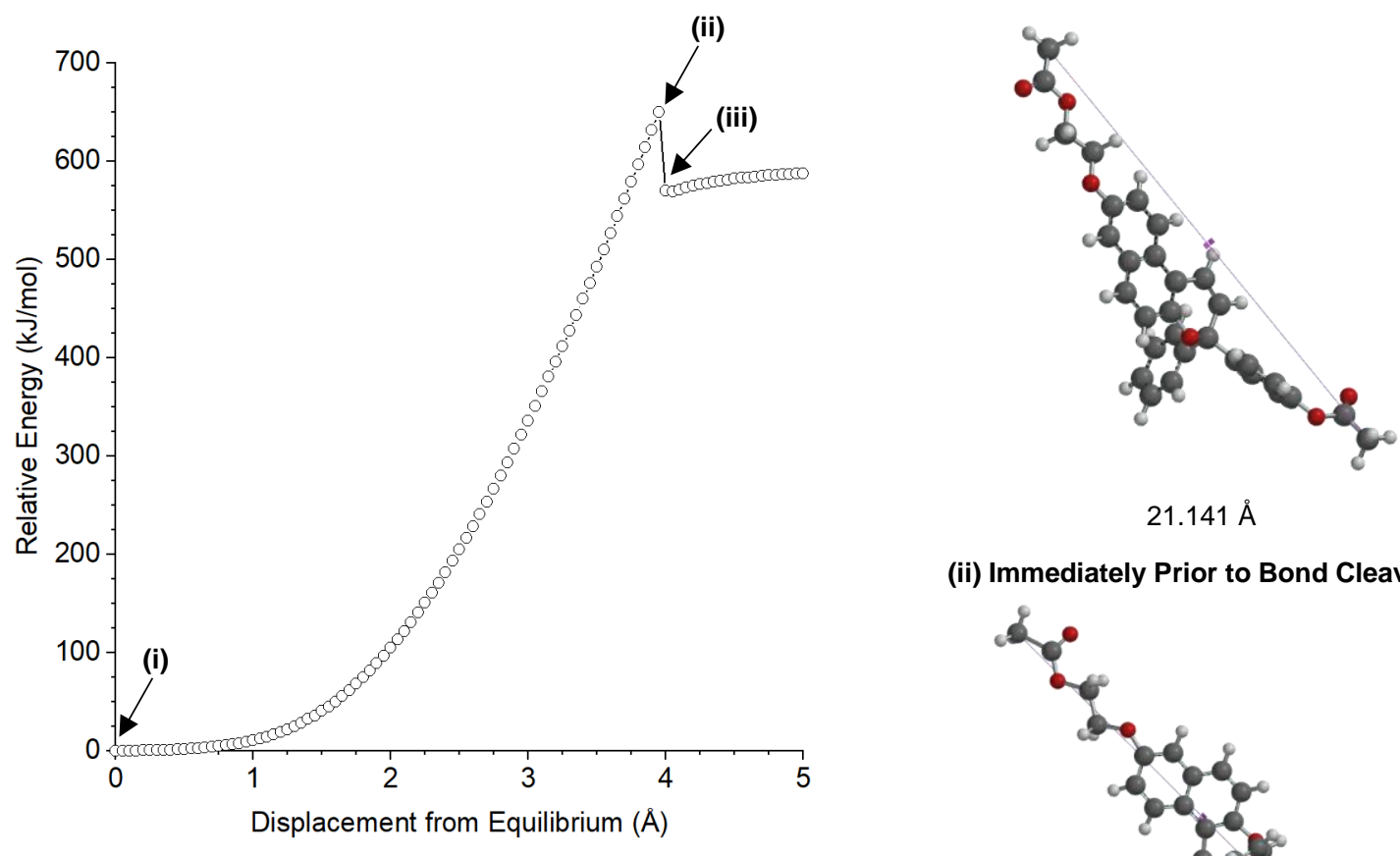

$21.141 \AA$

(ii) Immediately Prior to Bond Cleavage

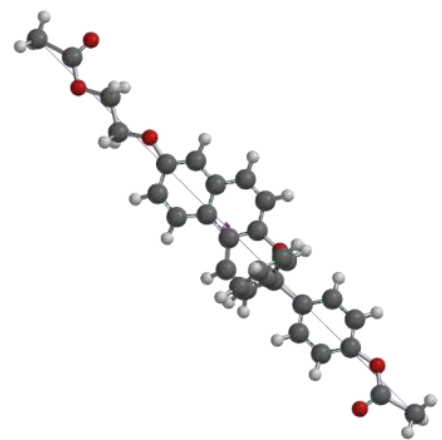

$25.091 \AA$

(iii) Immediately After Bond Cleavage

Force-Bond Angle $56^{\circ}$

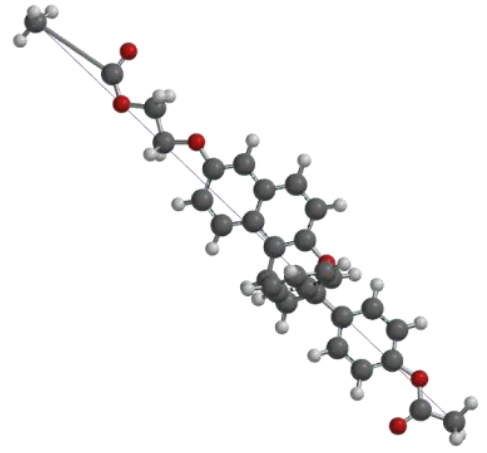

$25.141 \AA$ 


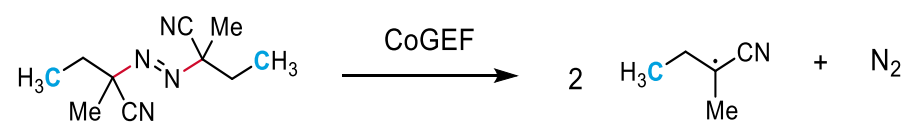

90

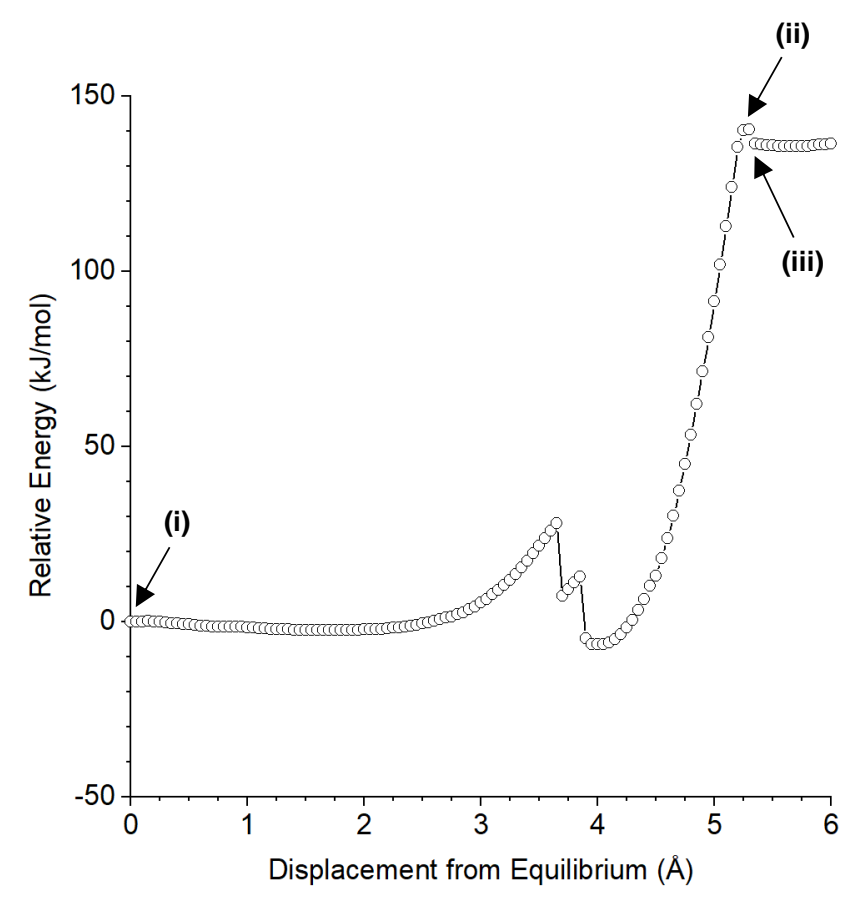

(i) Equilibrium Geometry

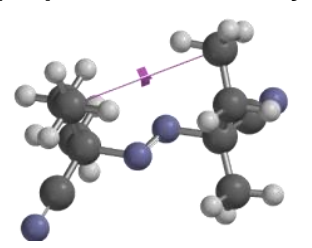

$4.452 \AA$

(ii) Immediately Prior to Bond Cleavage

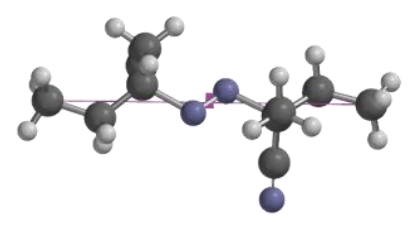

$9.652 \AA$

(iii) Immediately After Bond Cleavage

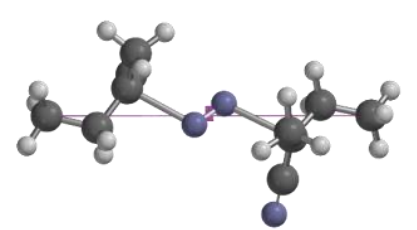

$9.702 \AA$

Summary of CoGEF Results

$$
\begin{array}{ll}
\boldsymbol{F}_{\max } & 3.7 \mathrm{nN} \\
\boldsymbol{E}_{\max } & 140 \mathrm{~kJ} / \mathrm{mol}
\end{array}
$$

Force-Bond Angle $29^{\circ}$ 


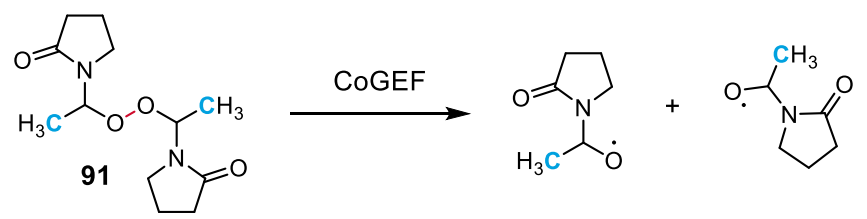

(i) Equilibrium Geometry
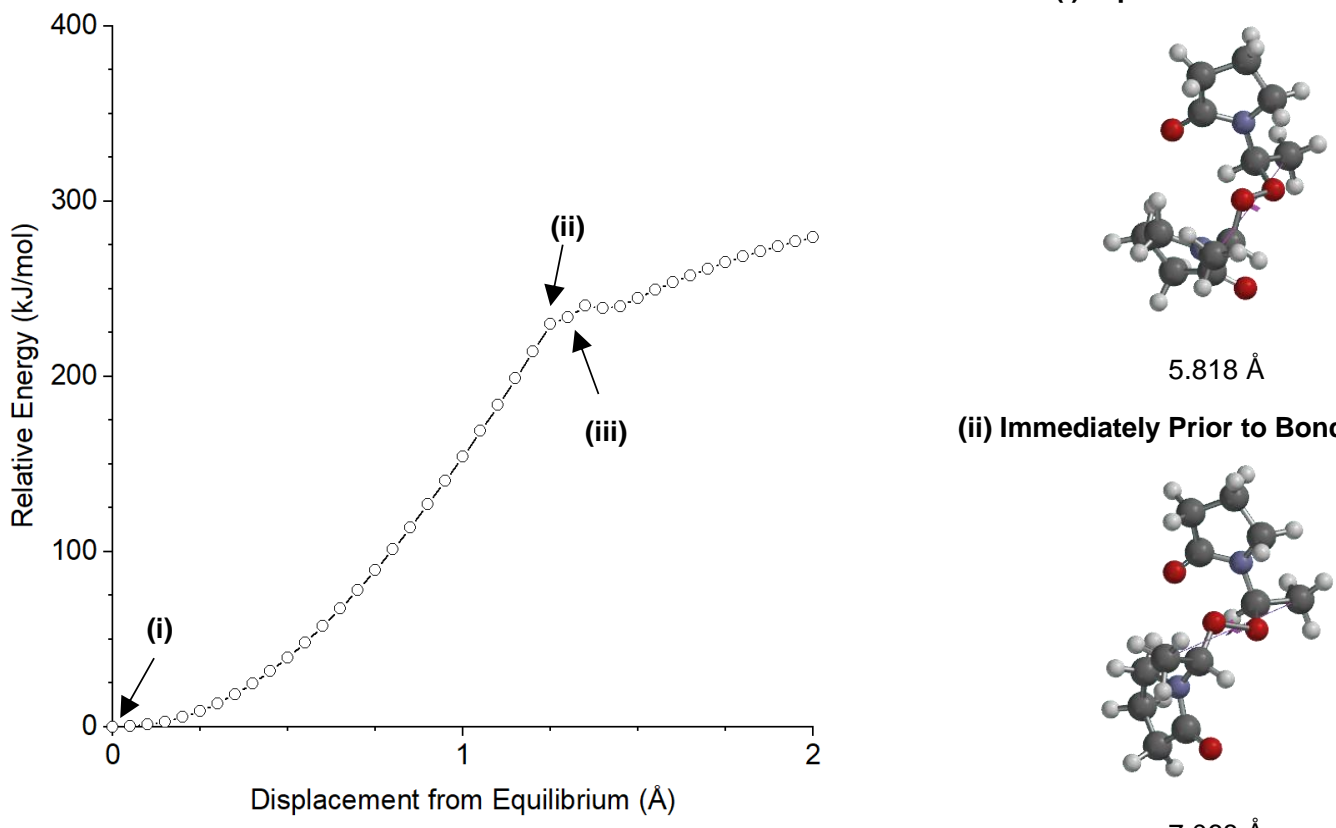

(ii) Immediately Prior to Bond Cleavage

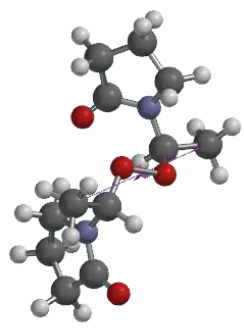

$7.068 \AA$

(iii) Immediately After Bond Cleavage

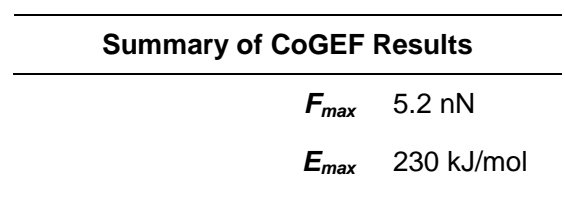

Force-Bond Angle $28^{\circ}$

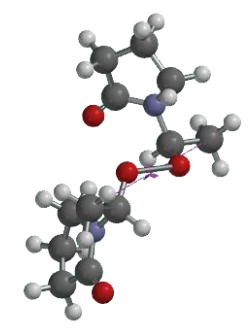

$7.118 \AA$ 


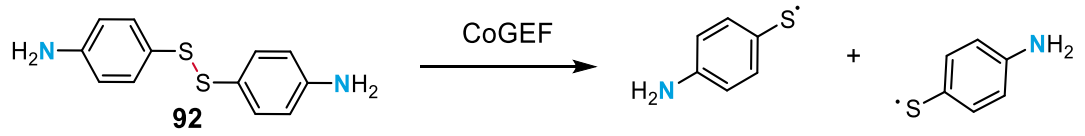

(i) Equilibrium Geometry
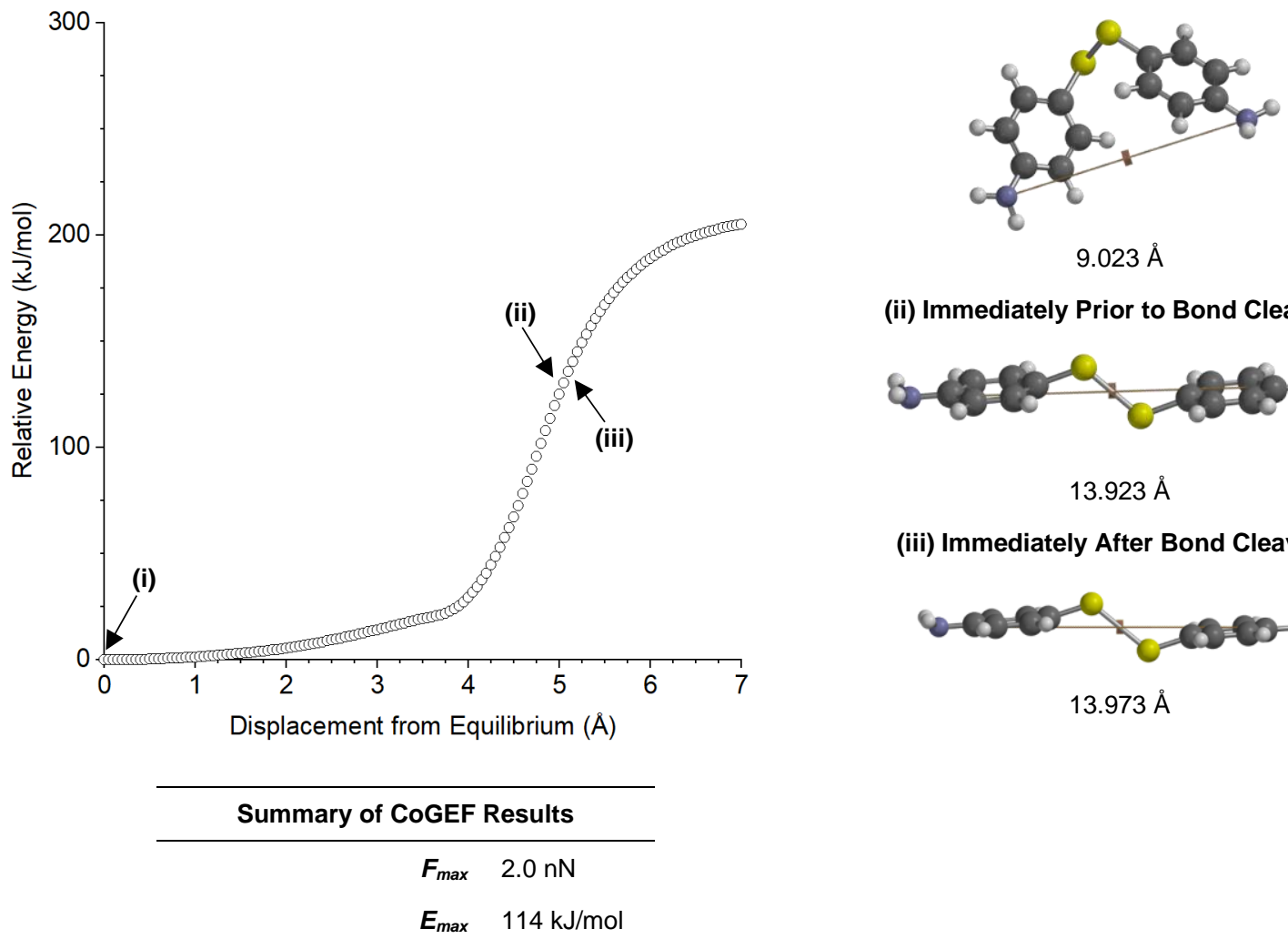

(ii) Immediately Prior to Bond Cleavage

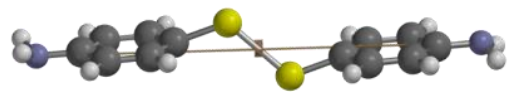

$13.923 \AA$

(iii) Immediately After Bond Cleavage

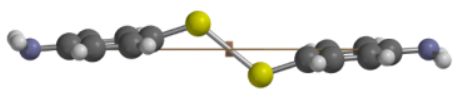

$13.973 \AA$

Force-Bond Angle $45^{\circ}$ 


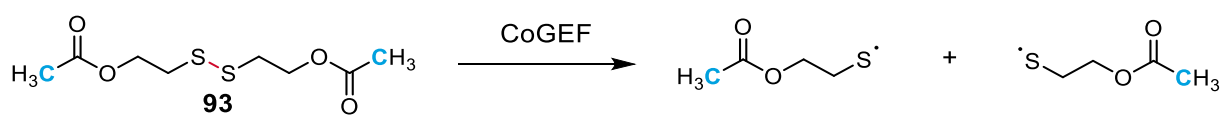

(i) Equilibrium Geometry
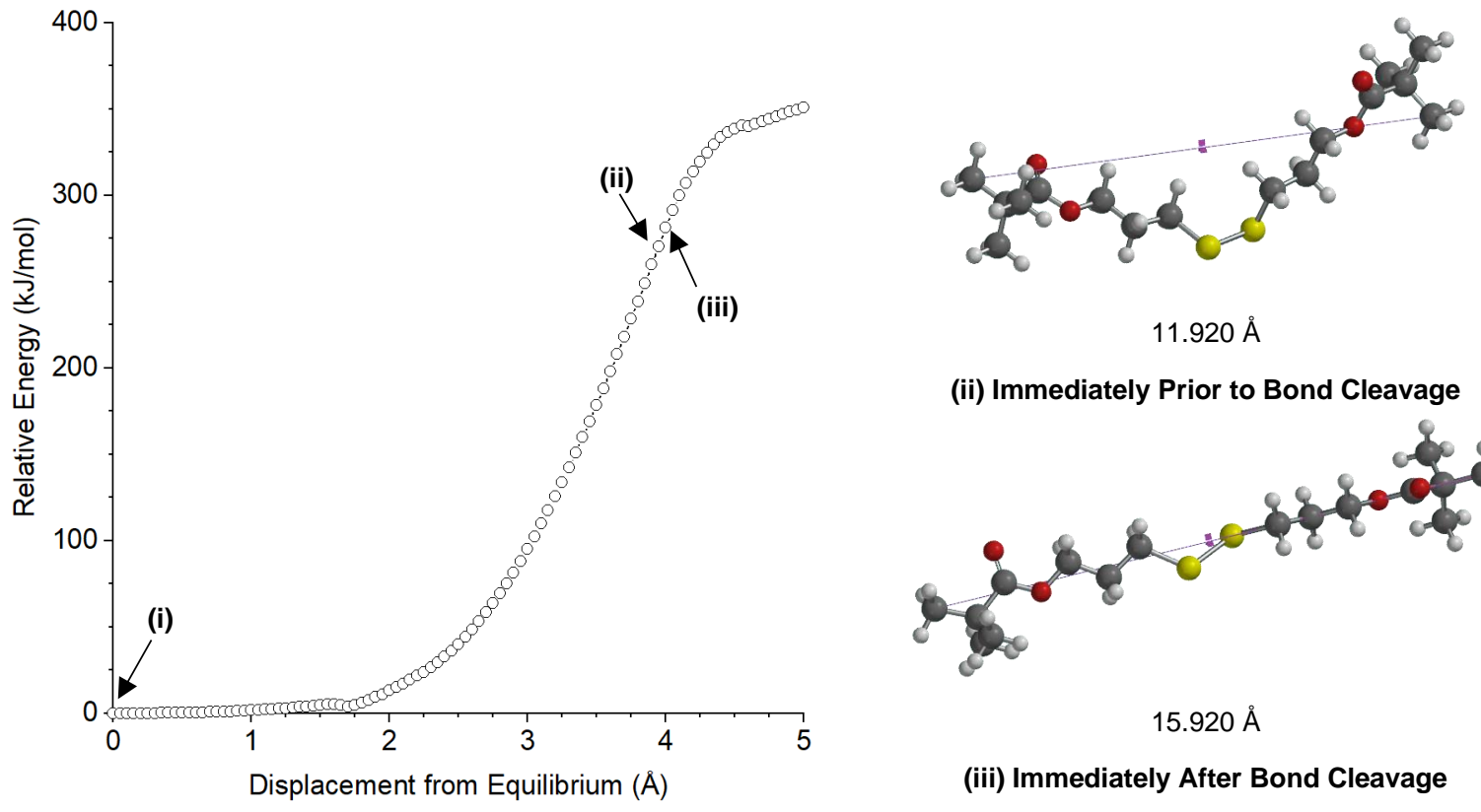

(ii) Immediately Prior to Bond Cleavage

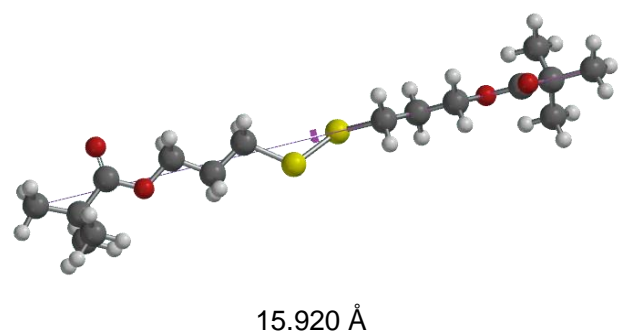

(iii) Immediately After Bond Cleavage

Summary of CoGEF Results

$$
\begin{array}{ll}
\boldsymbol{F}_{\text {max }} & 3.6 \mathrm{nN} \\
\boldsymbol{E}_{\text {max }} & 271 \mathrm{~kJ} / \mathrm{mol}
\end{array}
$$

Force-Bond Angle $29^{\circ}$

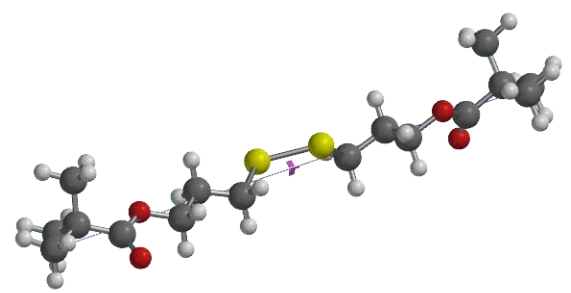

$15.970 \AA$ 

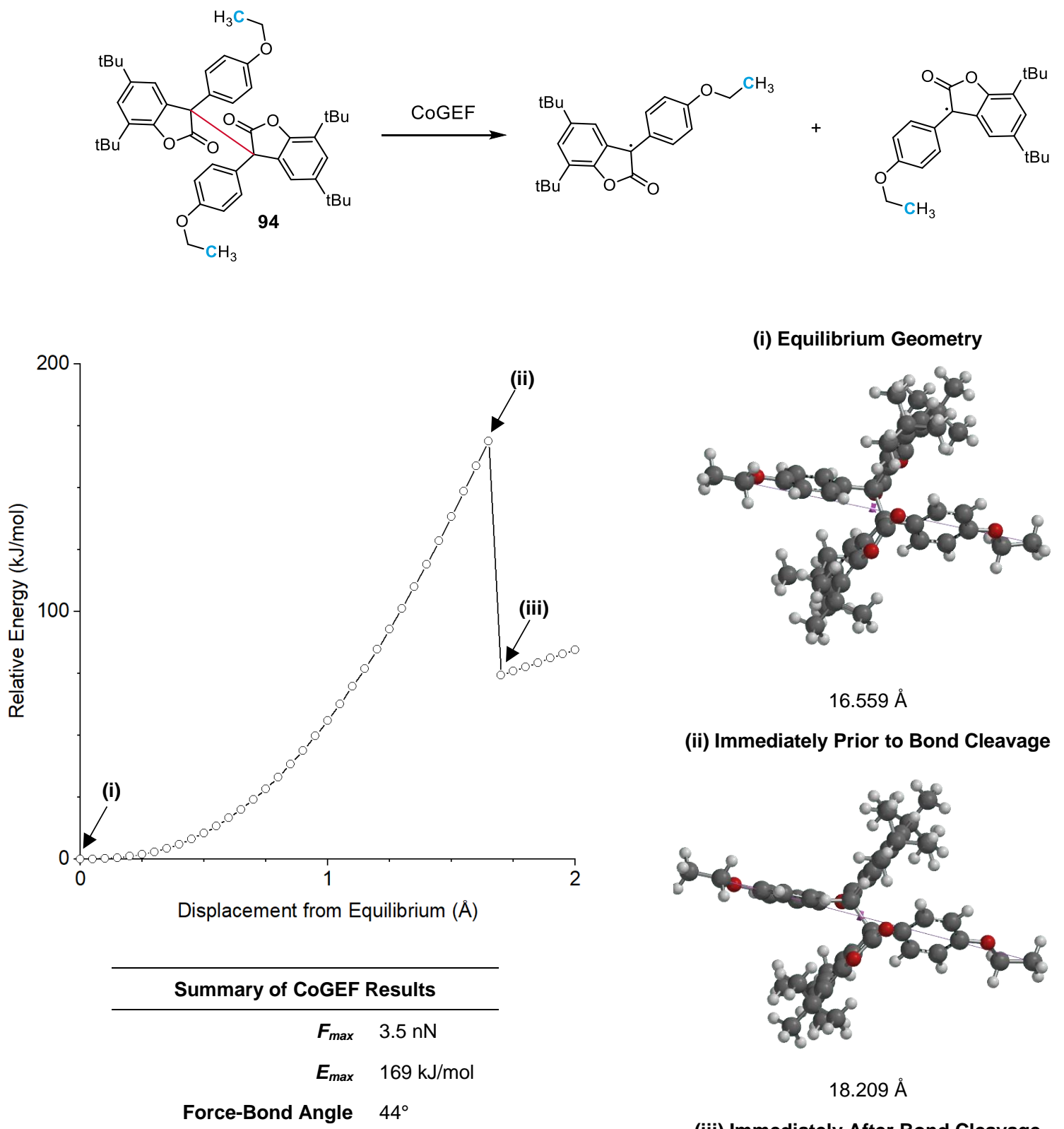

(i) Equilibrium Geometry

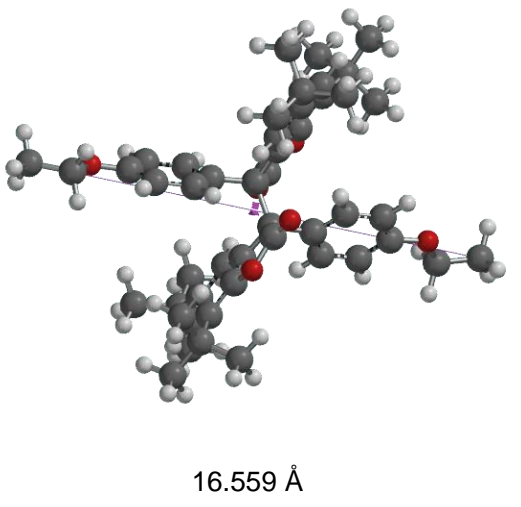

(ii) Immediately Prior to Bond Cleavage

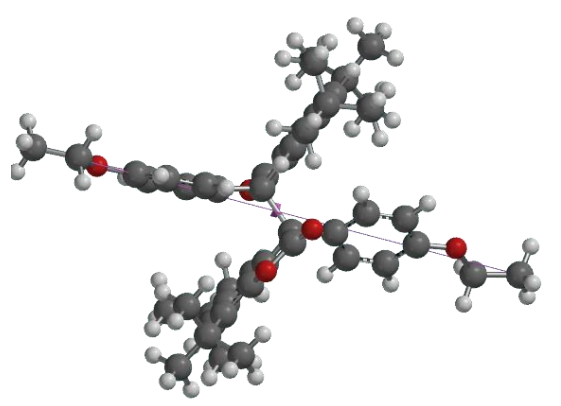

$18.209 \AA$

(iii) Immediately After Bond Cleavage

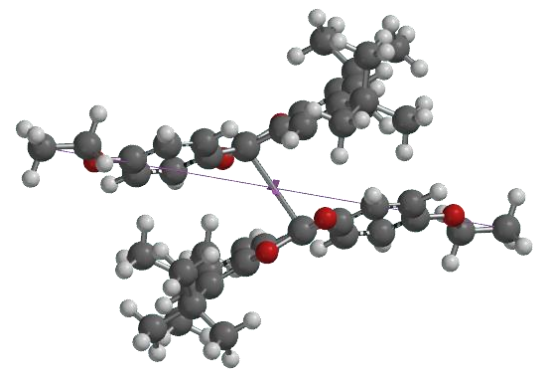

$18.259 \AA$ 

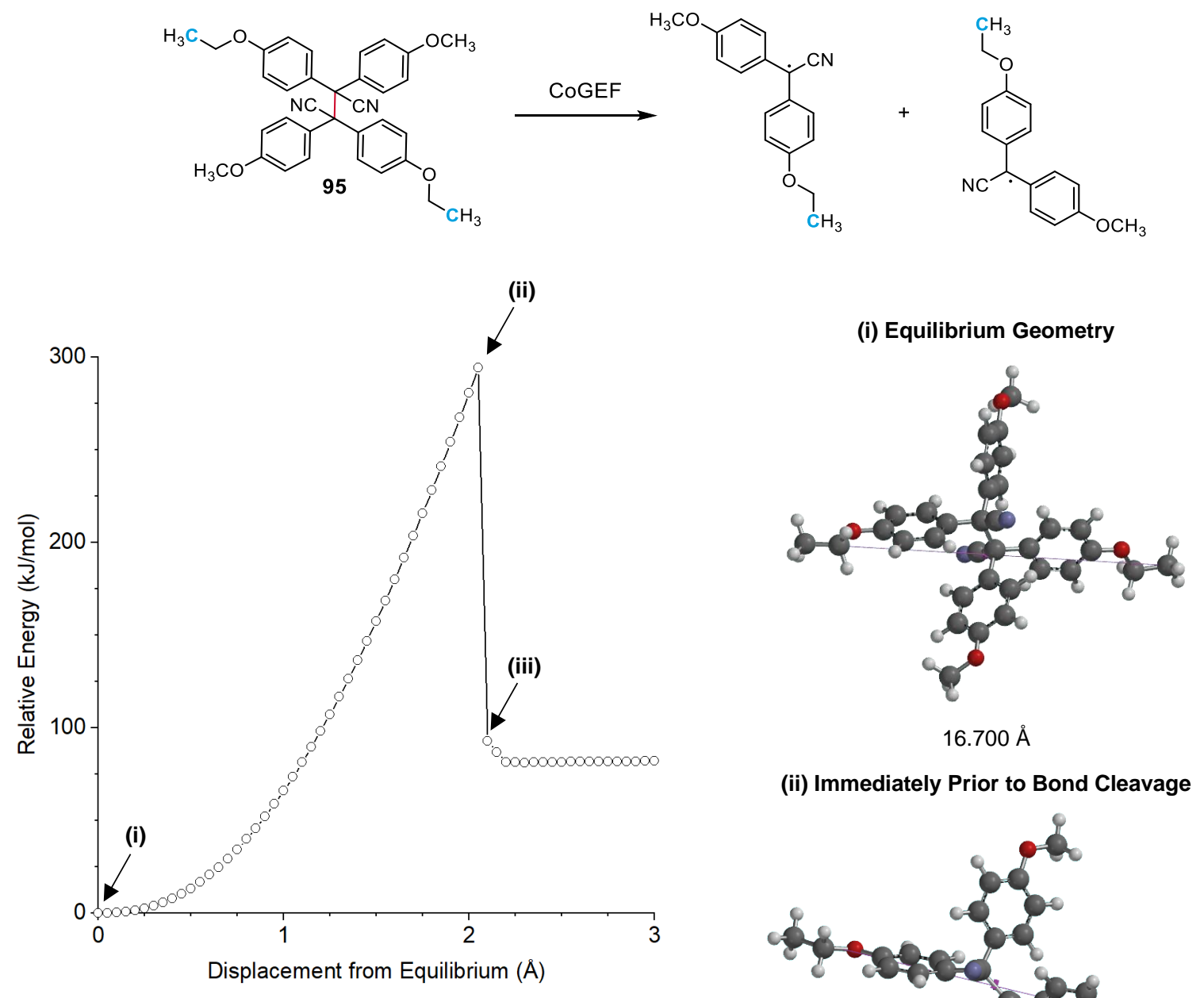

(ii) Immediately Prior to Bond Cleavage

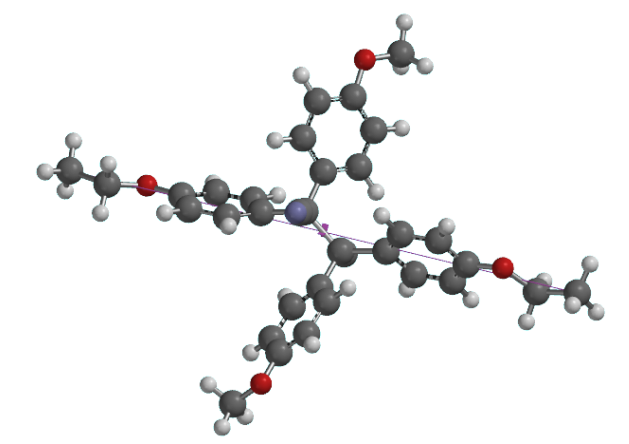

$18.750 \AA$

Force-Bond Angle $41^{\circ}$

(iii) Immediately After Bond Cleavage

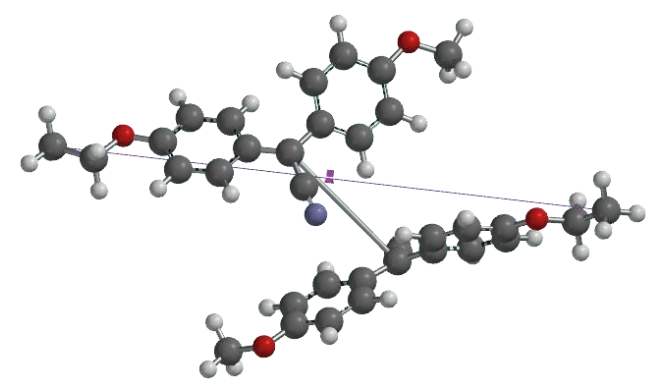

$18.800 \AA$ 


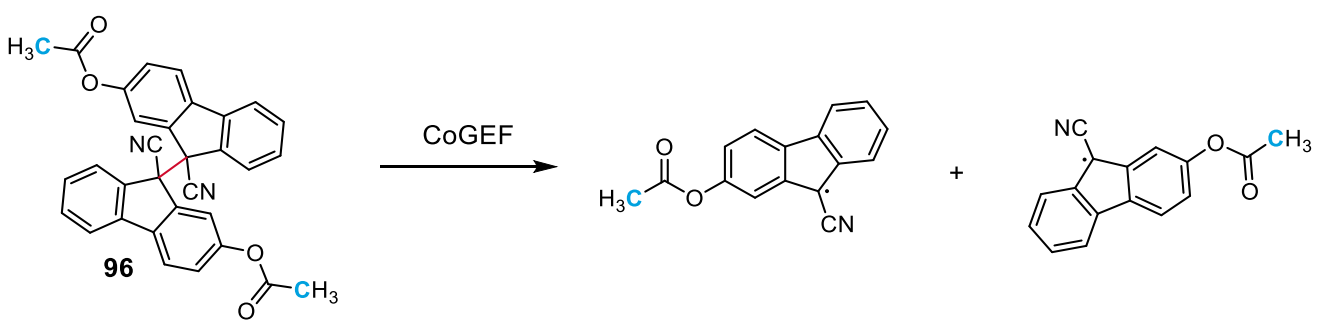

(i) Equilibrium Geometry
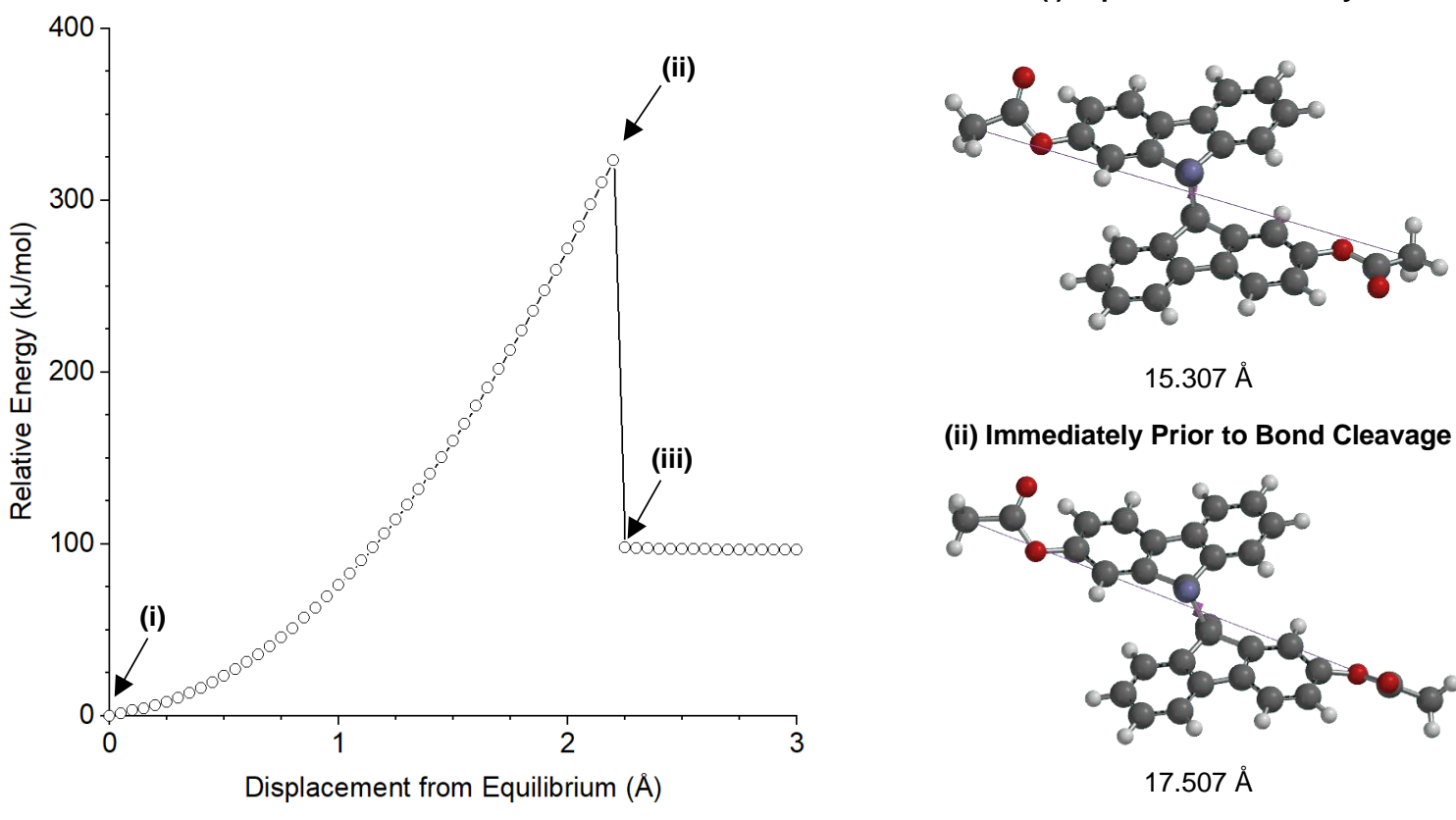

$15.307 \AA$

(ii) Immediately Prior to Bond Cleavage

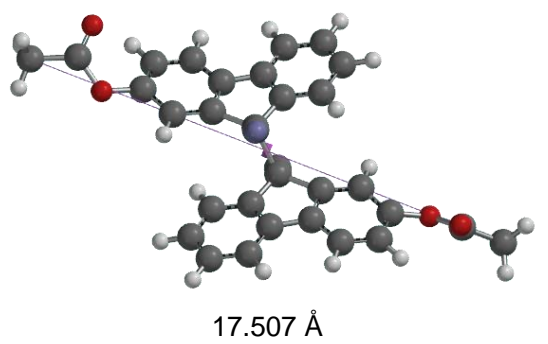

(iii) Immediately After Bond Cleavage

Summary of CoGEF Results

$F_{\text {max }} 4.3 \mathrm{nN}$

$E_{\max } \quad 326 \mathrm{~kJ} / \mathrm{mol}$

Force-Bond Angle $46^{\circ}$

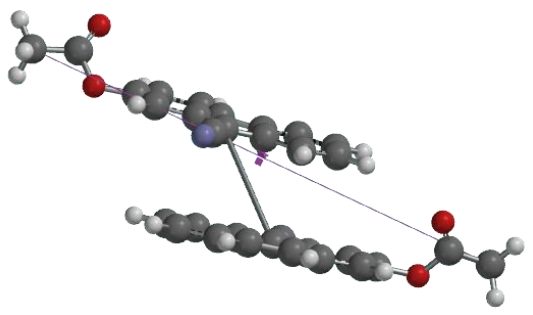

$17.557 \AA$ 

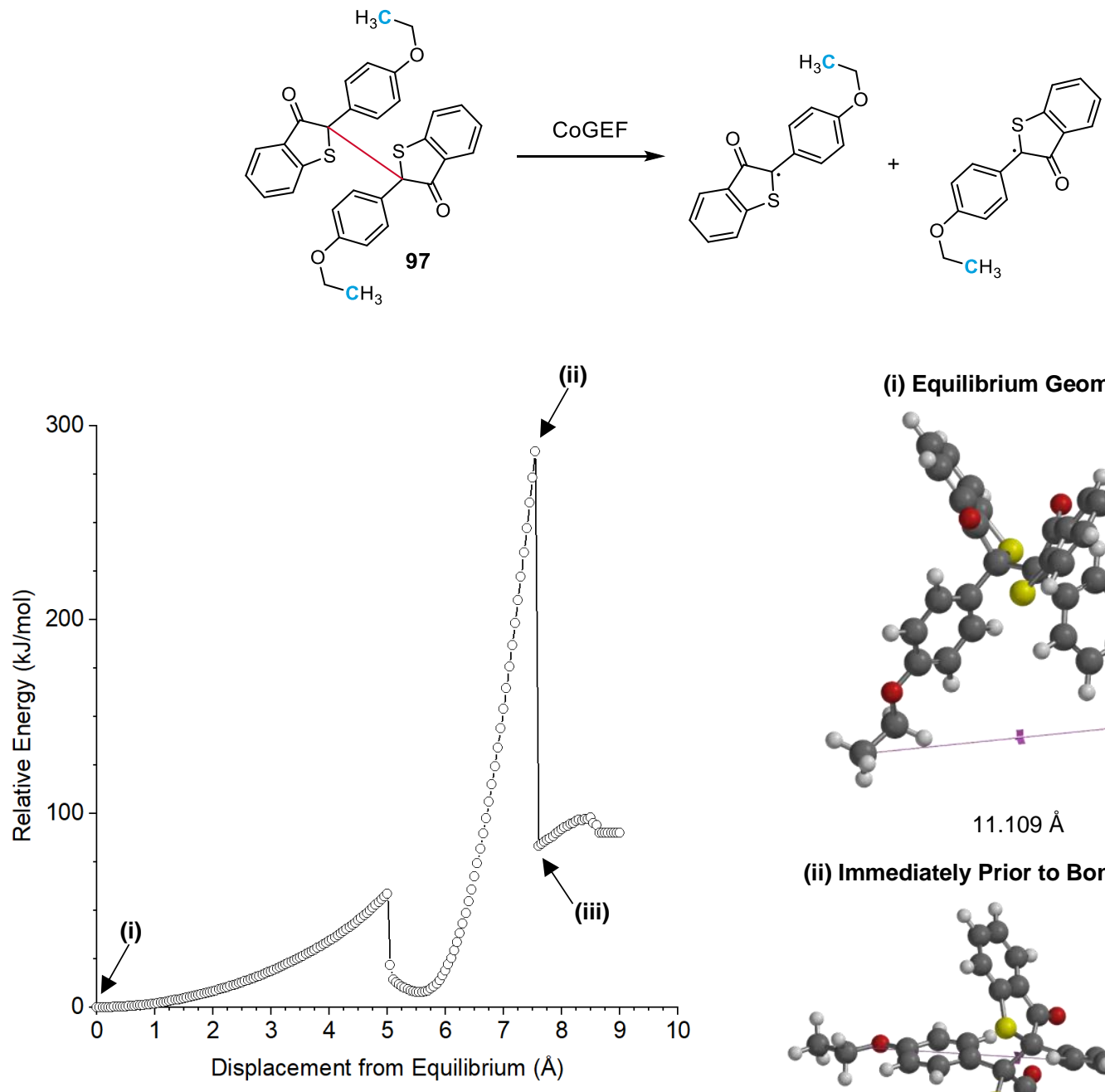

Summary of CoGEF Results

$\boldsymbol{F}_{\max } \quad 4.4 \mathrm{nN}$

$E_{\max } 287 \mathrm{~kJ} / \mathrm{mol}$

Force-Bond Angle $\quad 41^{\circ}$

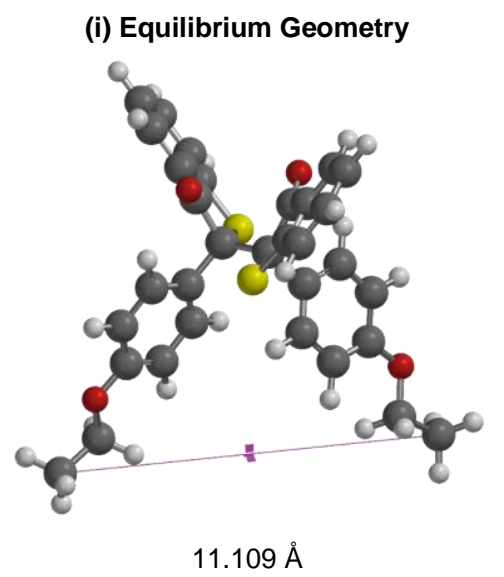

(ii) Immediately Prior to Bond Cleavage

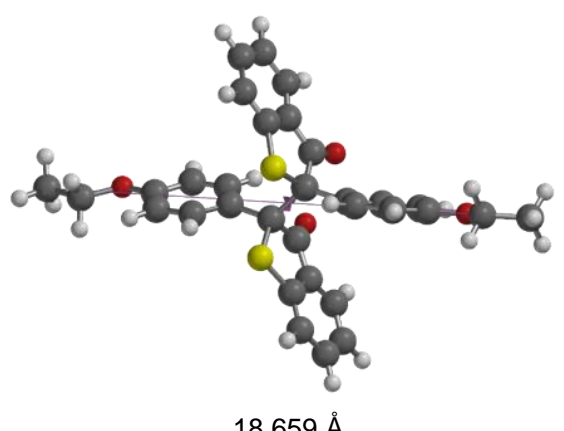

(iii) Immediately After Bond Cleavage

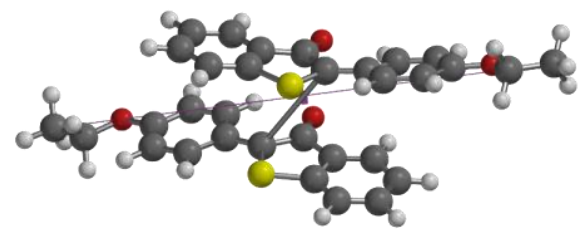

$18.709 \AA$ 


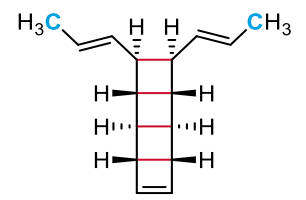

98

(i) Equilibrium Geometry

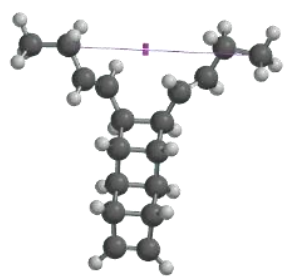

$6.269 \AA$

(ii) Immediately Prior to First Bond

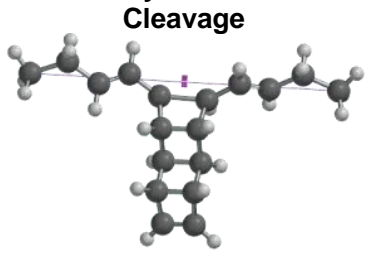

$10.019 \AA$

(iii) Immediately After First Bond Cleavage

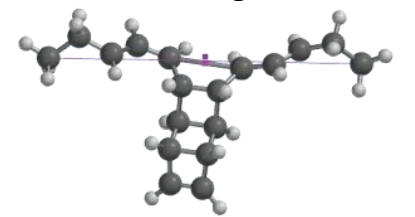

$10.069 \AA$

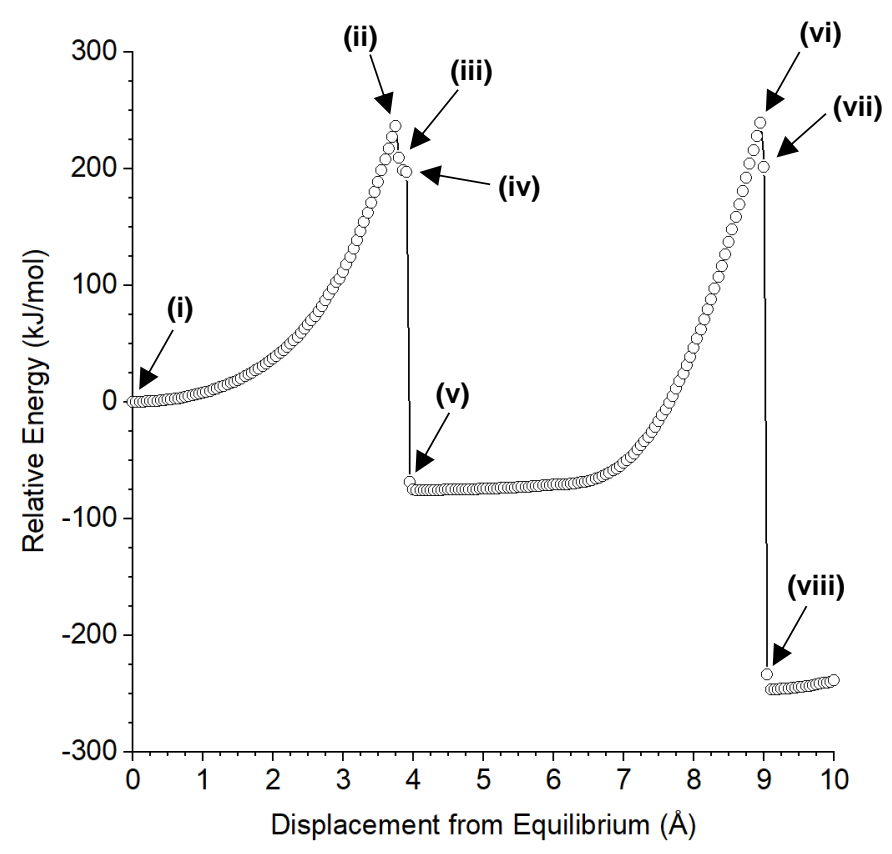

$15.219 \AA$

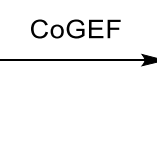

(iv) Immediately Prior to Second Bond Cleavage

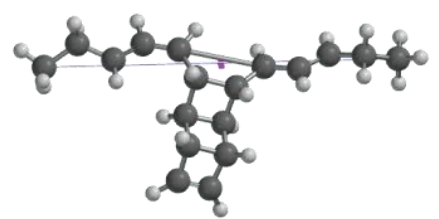

$10.169 \AA$

(v) Immediately After Second Bond Cleavage

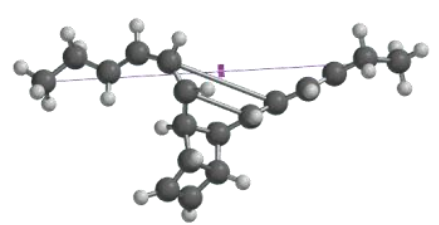

$10.219 \AA$

(vi) Immediately Prior to Third Bond Cleavage

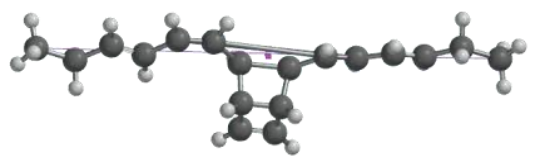

(vii) Immediately After Third Bond Cleavage

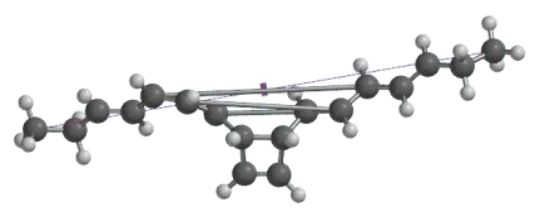

$15.269 \AA$

(viii) Immediately After Fourth Bond Cleavage

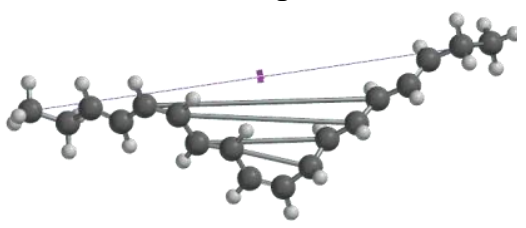

$15.319 \AA$

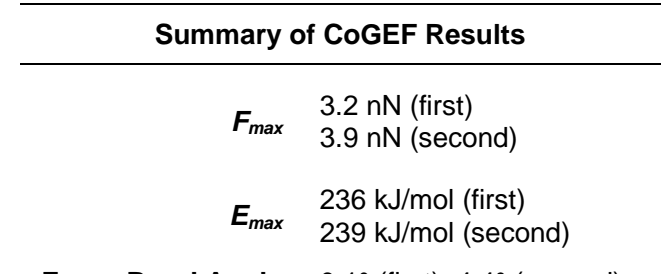

Force-Bond Angle $\quad 0.1^{\circ}$ (first), $4.4^{\circ}$ (second) 


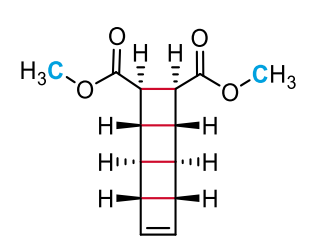

99

(i) Equilibrium Geometry

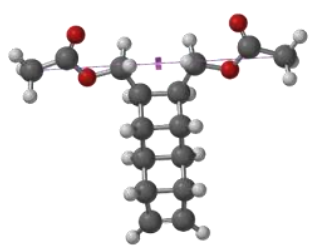

$10.304 \AA$

(ii) Immediately Prior to First Bond Cleavage

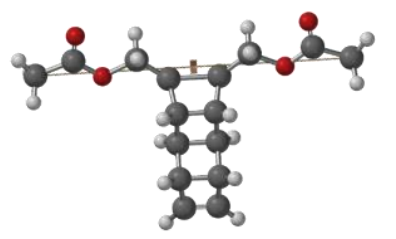

$12.554 \AA$

(iii) Immediately After First Bond Cleavage

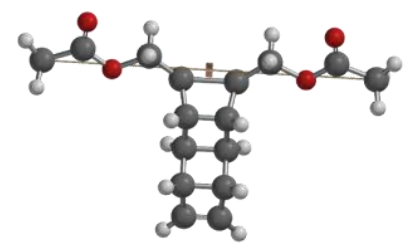

$12.604 \AA$

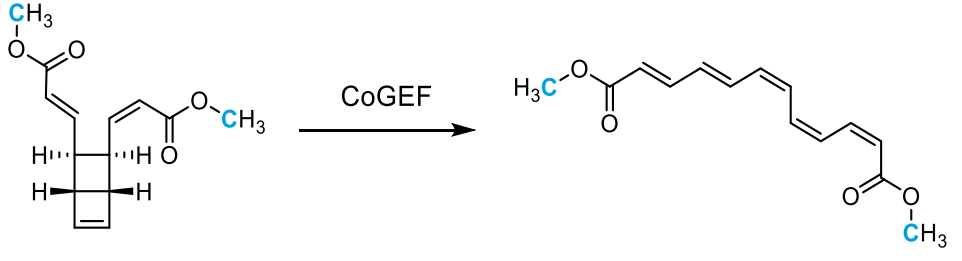

(iv) Immediately Prior to Second Bond Cleavage

(vii) Immediately After Third Bond Cleavage

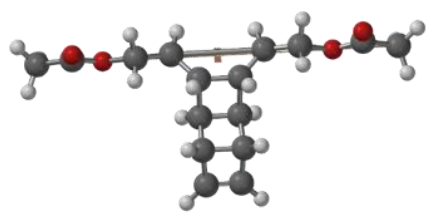

$13.754 \AA$

(v) Immediately After Second Bond Cleavage

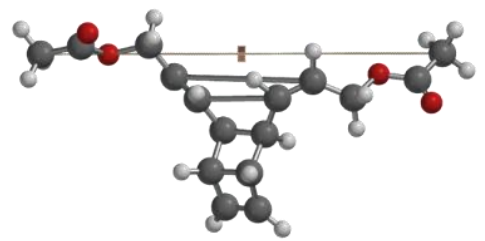

$13.804 \AA$

(vi) Immediately Prior to Third Bond Cleavage

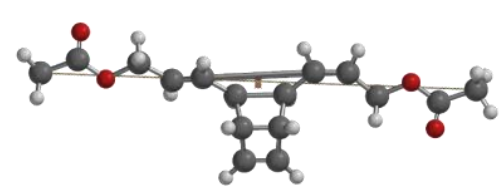

$17.554 \AA$

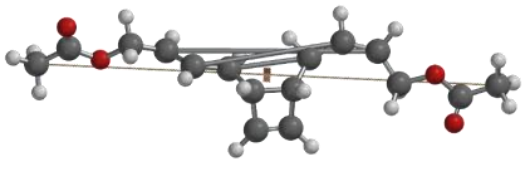

$17.604 \AA$

(viii) Immediately After Fourth Bond Cleavage

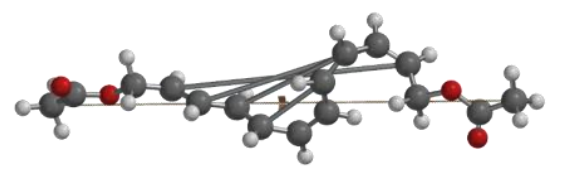

$17.654 \AA$

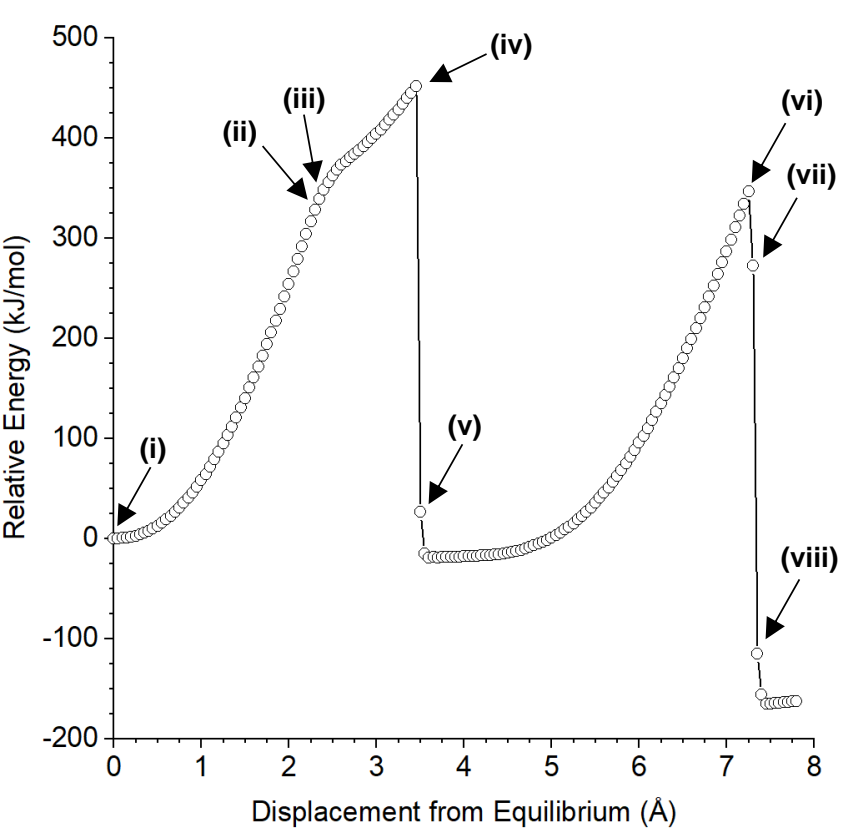




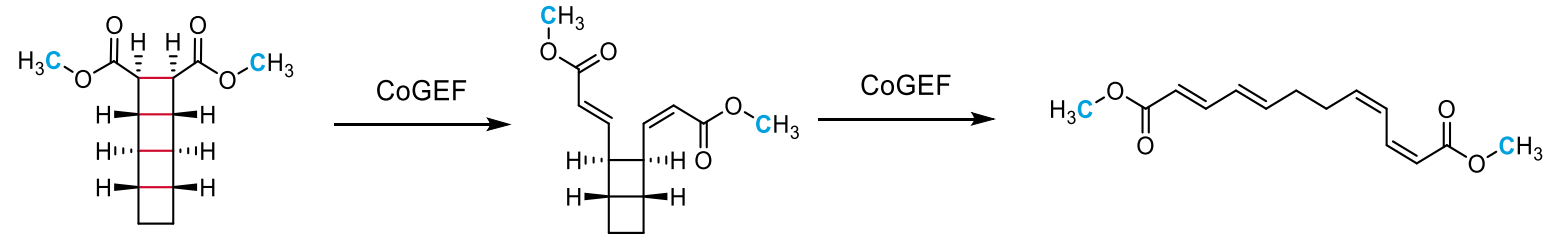

100

(i) Equilibrium Geometry

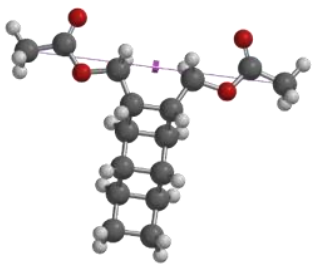

$10.308 \AA$

(ii) Immediately Prior to First Bond Cleavage

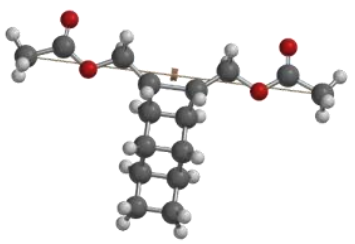

$12.458 \AA$

(iii) Immediately After First Bond Cleavage

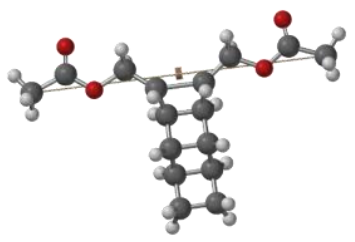

$12.508 \AA$ (iv) Immediately Prior to Second Bond Cleavage

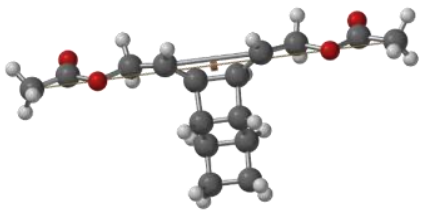

$14.208 \AA$

(v) Immediately After Second Bond Cleavage

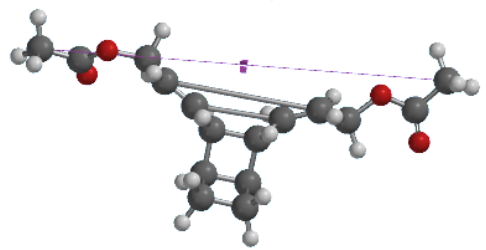

$14.258 \AA$

(vi) Immediately Prior to Third Bond Cleavage

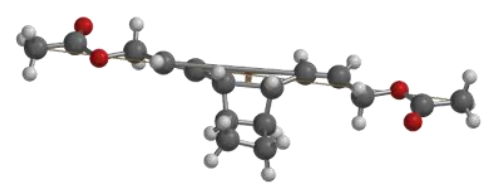

$17.508 \AA$ (vii) Immediately After Third Bond Cleavage

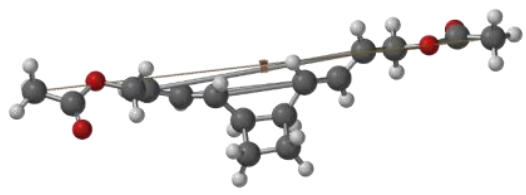

$17.558 \AA$

(viii) Immediately After Fourth Bond Cleavage

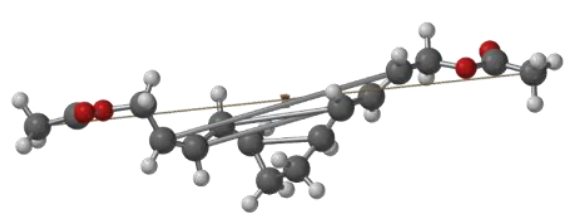

$17.708 \AA$

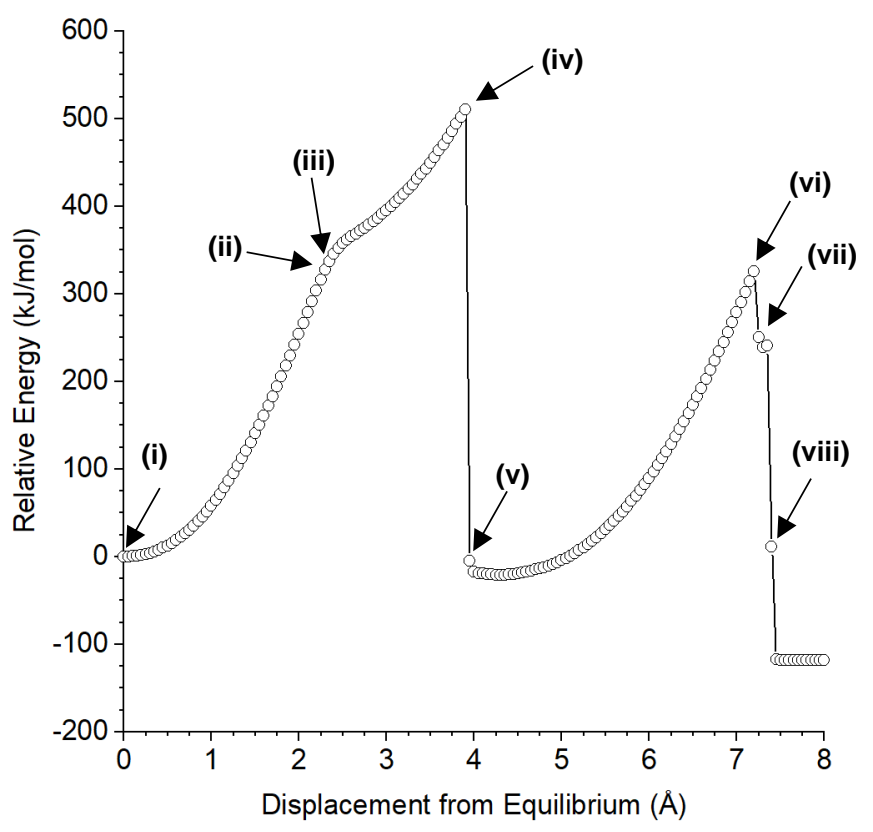




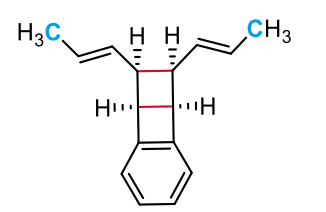

101

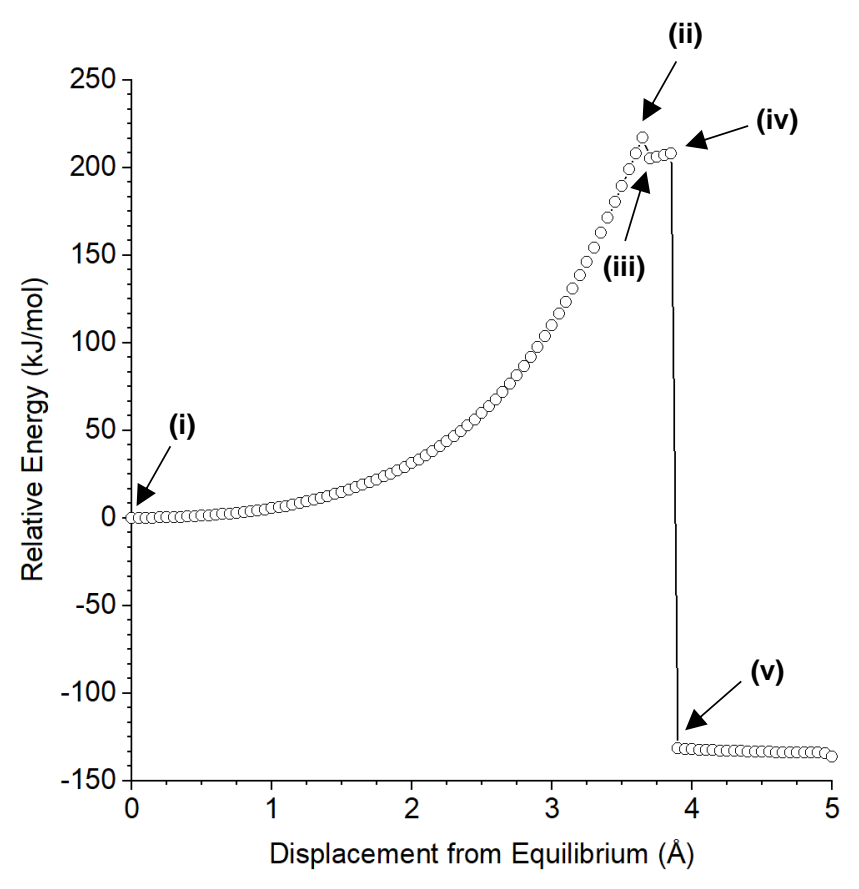

Summary of CoGEF Results

$\boldsymbol{F}_{\max } \quad 3.1 \mathrm{nN}$

$E_{\max } 207 \mathrm{~kJ} / \mathrm{mol}$

Force-Bond Angle $\quad 0.1^{\circ}$

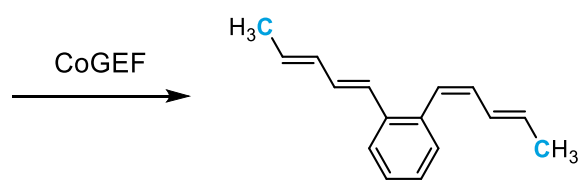

(i) Equilibrium Geometry

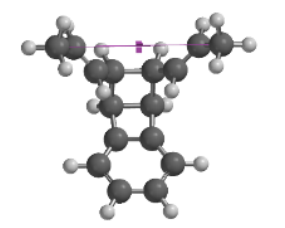

$6.368 \AA$

(ii) Immediately Prior to First Bond Cleavage

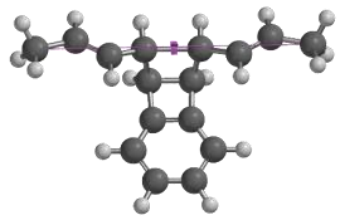

$10.018 \AA$

(iii) Immediately After First Bond Cleavage

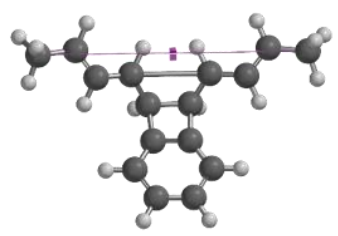

$10.068 \AA$

(iv) Immediately Prior to Second Bond Cleavage

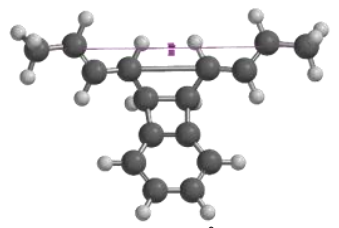

$10.218 \AA$

(v) Immediately After Second Bond Cleavage

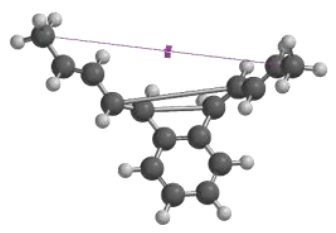

$10.268 \AA$ 

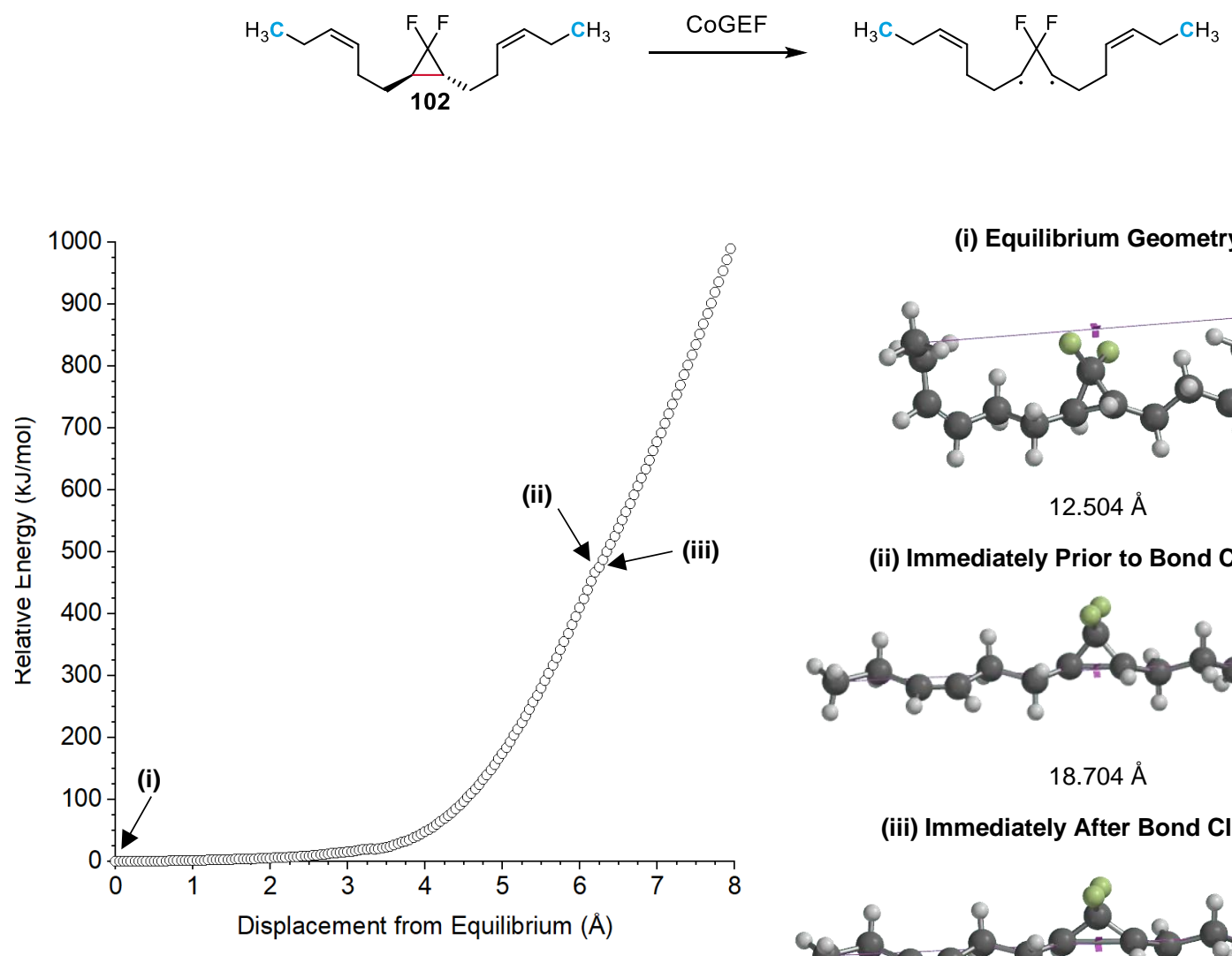

(i) Equilibrium Geometry

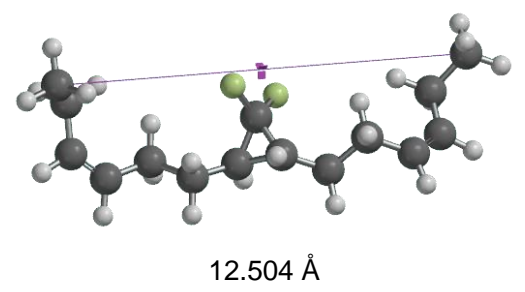

(ii) Immediately Prior to Bond Cleavage

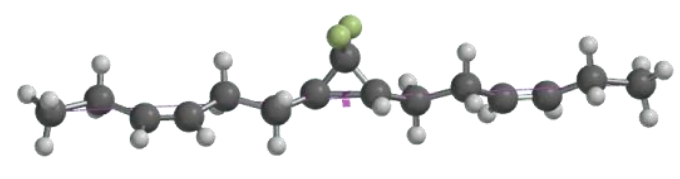

$18.704 \AA$

(iii) Immediately After Bond Cleavage

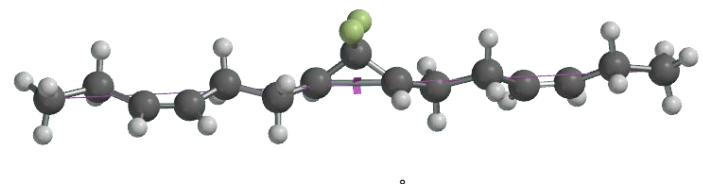

$18.754 \AA$

Summary of CoGEF Results

$\boldsymbol{F}_{\text {max }} \quad 4.8 \mathrm{nN}$

$E_{\max } \quad 466 \mathrm{~kJ} / \mathrm{mol}$

Force-Bond Angle $21^{\circ}$ 


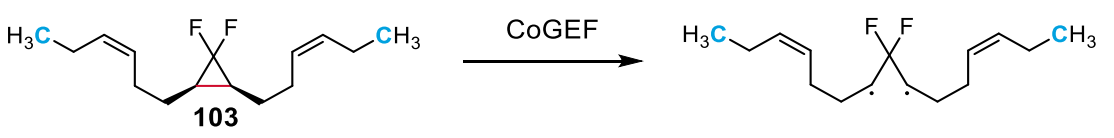

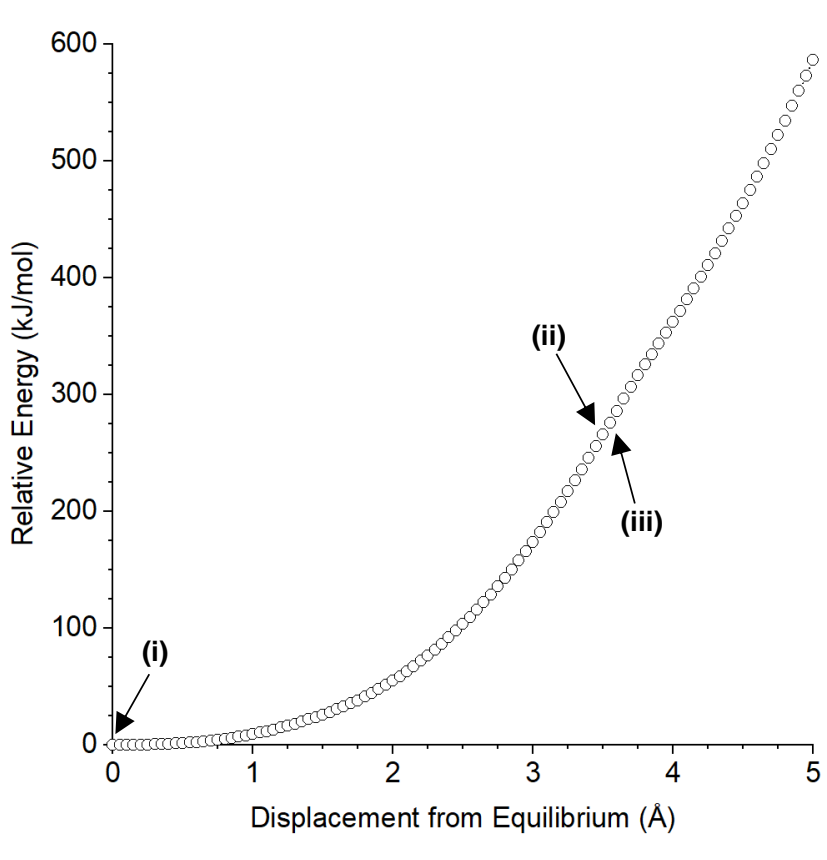

Summary of CoGEF Results

$$
\begin{array}{ll}
\boldsymbol{F}_{\max } & 3.4 \mathrm{nN} \\
\boldsymbol{E}_{\max } & 292 \mathrm{~kJ} / \mathrm{mol}
\end{array}
$$

Force-Bond Angle $\quad 0.0^{\circ}$ (i) Equilibrium Geometry

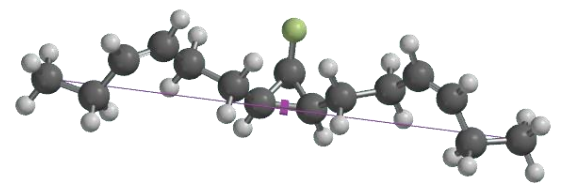

$14.312 \AA$

(ii) Immediately Prior to Bond Cleavage

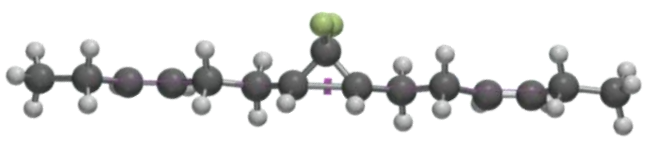

$17.912 \AA$

(iii) Immediately After Bond Cleavage

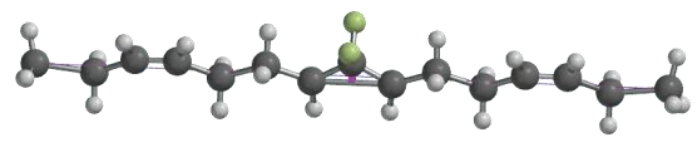

$17.962 \AA$ 


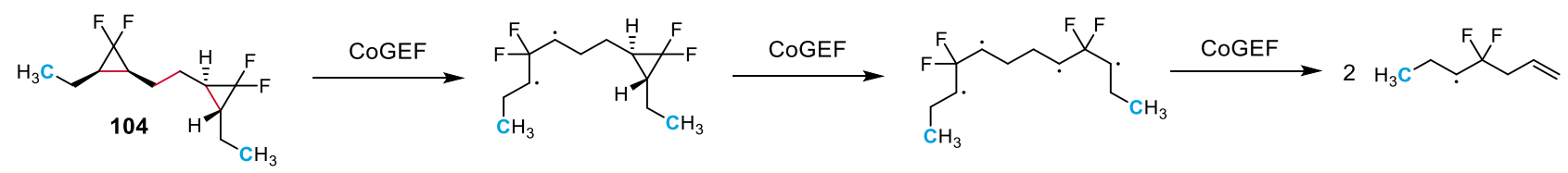

(i) Equilibrium Geometry

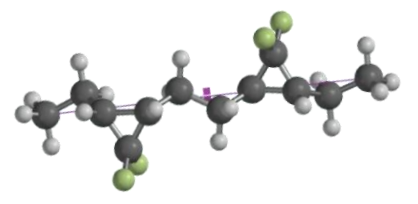

$10.904 \AA$

(ii) Immediately Prior to First Bond Cleavage

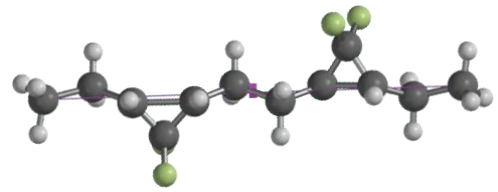

$13.104 \AA$

(iii) Immediately After First Bond Cleavage

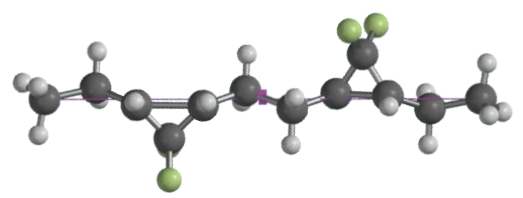

$13.154 \AA$

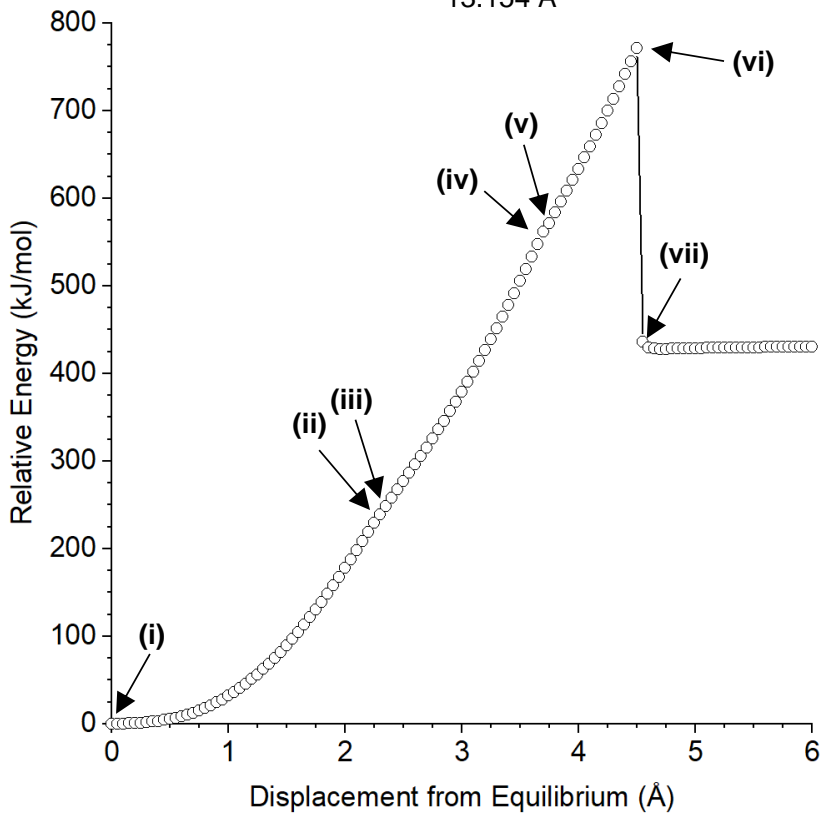

(iv) Immediately Prior to Second Bond Cleavage

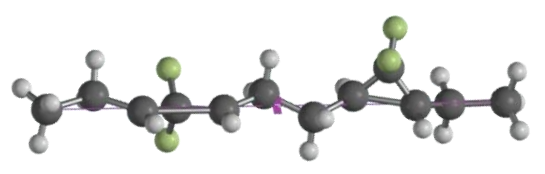

$14.604 \AA$

(v) Immediately After Second Bond Cleavage

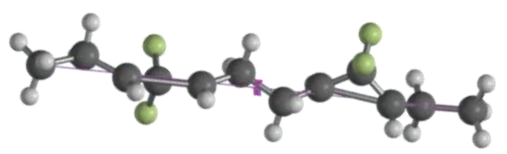

$14.654 \AA$

(vi) Immediately Prior to Disproportionation

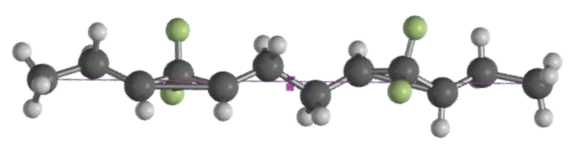

$15.404 \AA$

(vii) Immediately After Disproportionation

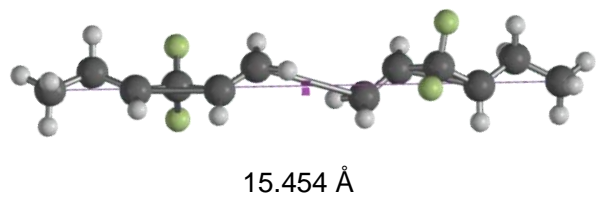

Summary of CoGEF Results

$$
\begin{array}{ll}
\boldsymbol{F}_{\text {max }} & 4.9 \mathrm{nN} \\
\boldsymbol{E}_{\max } & 771 \mathrm{~kJ} / \mathrm{mol}
\end{array}
$$

Force-Bond Angle $1.3^{\circ}$ 

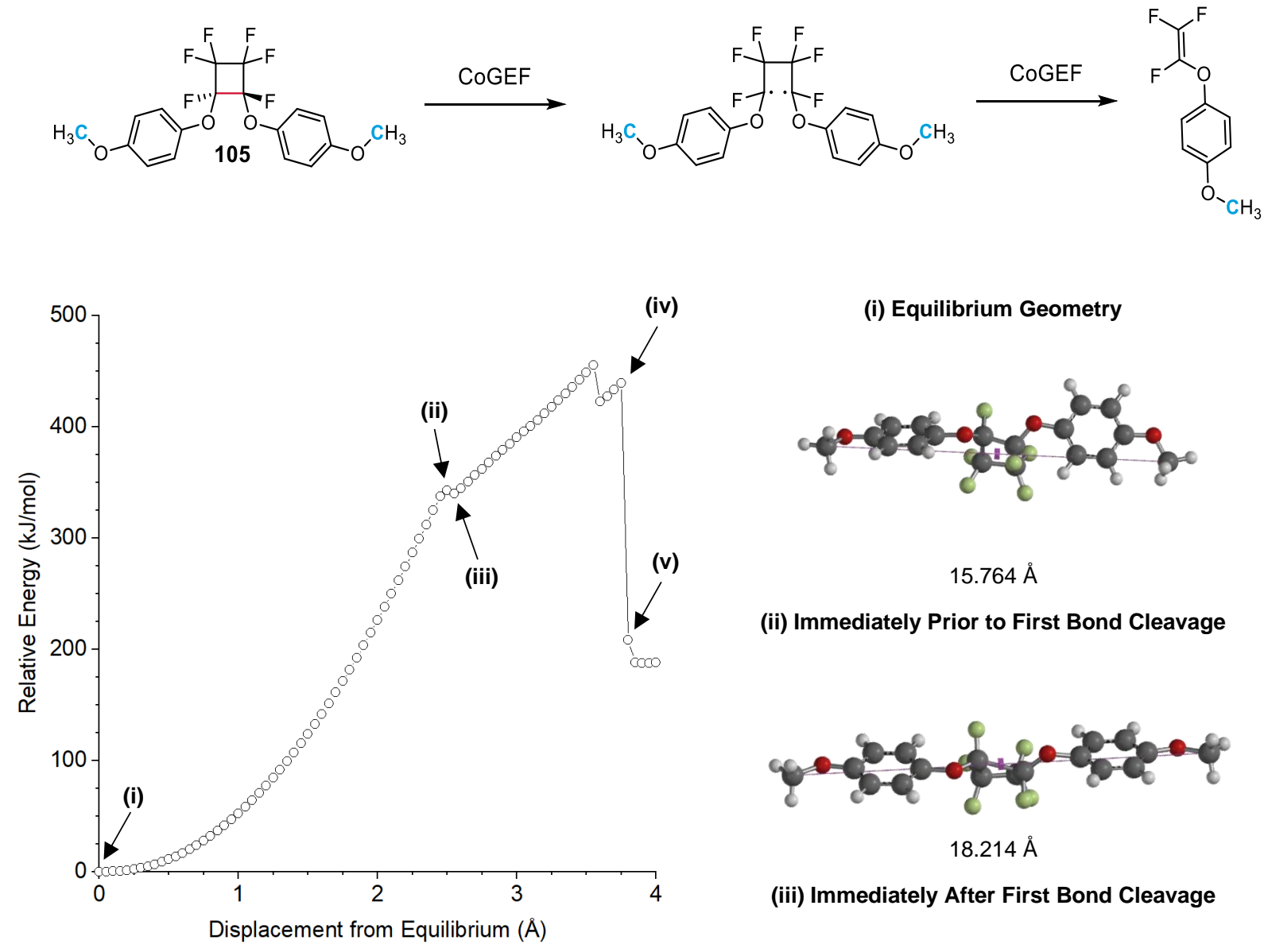

(i) Equilibrium Geometry

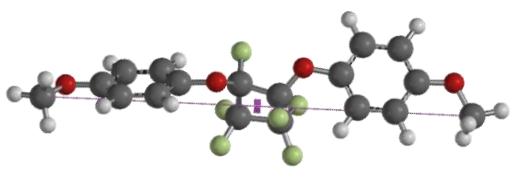

(v)

$15.764 \AA$

(ii) Immediately Prior to First Bond Cleavage

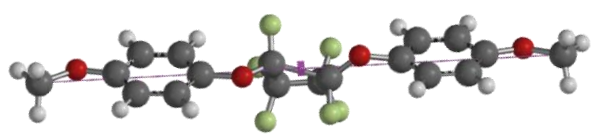

$18.214 \AA$

(iii) Immediately After First Bond Cleavage

Summary of CoGEF Results

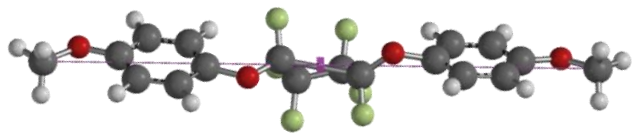

$\boldsymbol{F}_{\max } \quad 4.2 \mathrm{nN}$

$18.264 \AA$

$E_{\max } \quad 455 \mathrm{~kJ} / \mathrm{mol}$

(iv) Immediately Before Second Bond Cleavage

Force-Bond Angle $17^{\circ}$

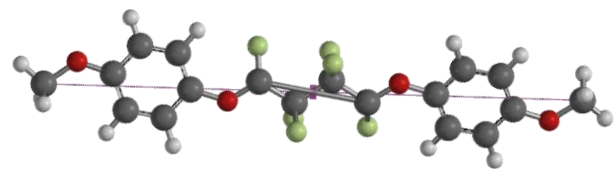

$19.514 \AA$

(v) Immediately After Second Bond Cleavage

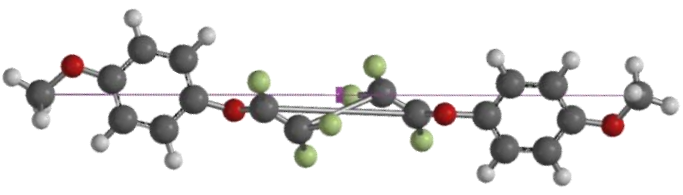

$19.562 \AA$ 

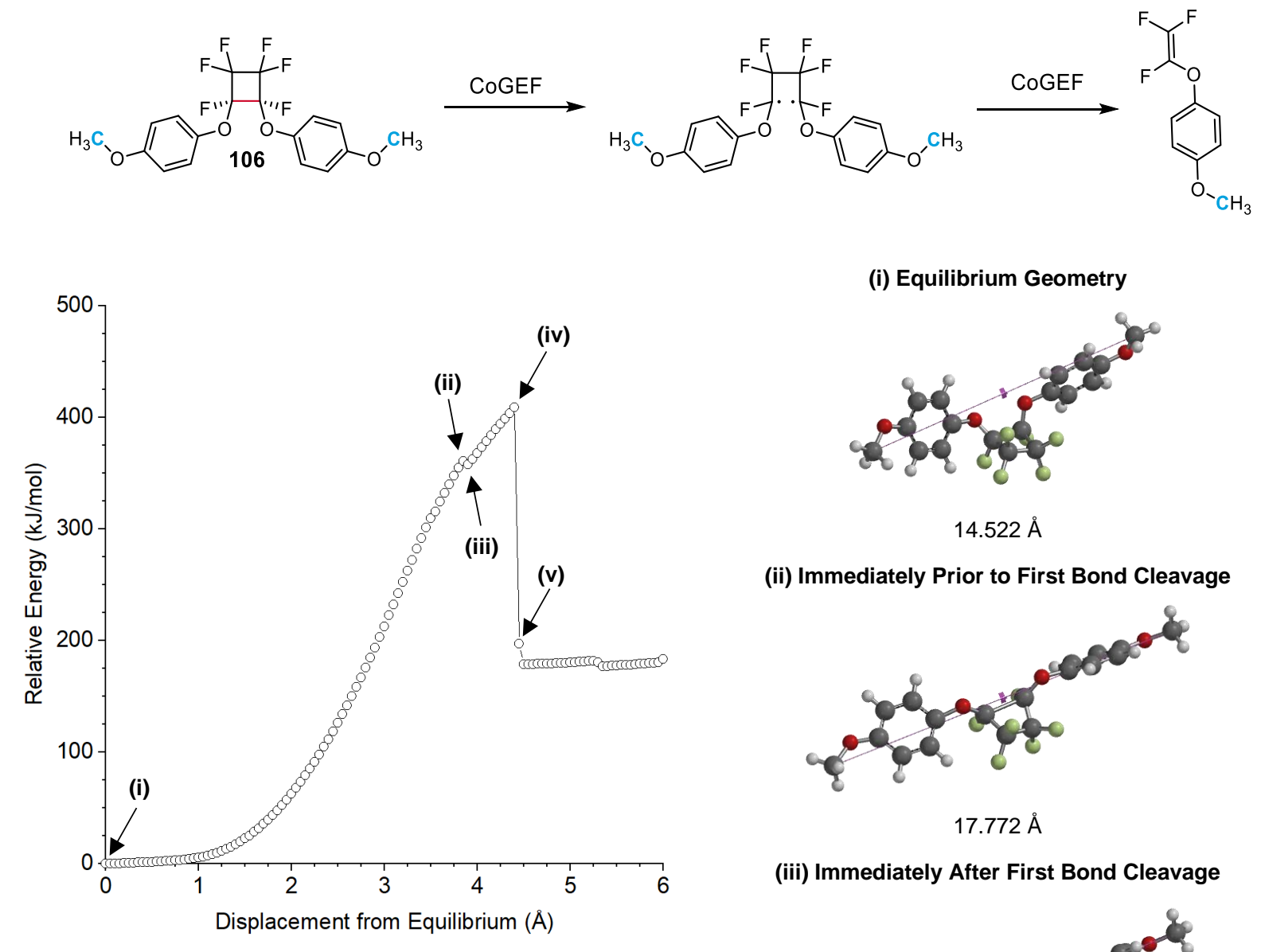

(i) Equilibrium Geometry

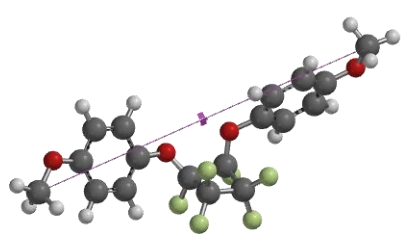

$14.522 \AA$

(ii) Immediately Prior to First Bond Cleavage

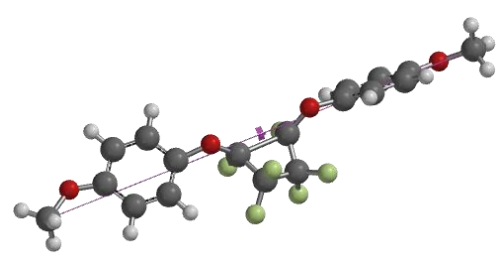

$17.772 \AA$

(iii) Immediately After First Bond Cleavage

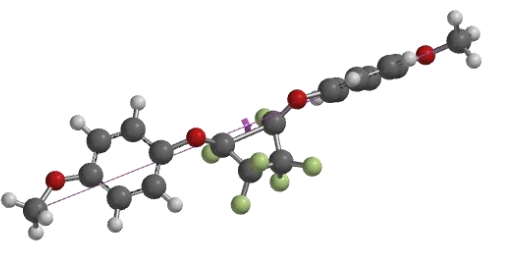

$17.822 \AA$

(iv) Immediately Before Second Bond Cleavage

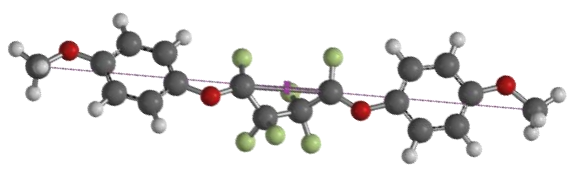

$18.922 \AA$

(v) Immediately After Second Bond Cleavage

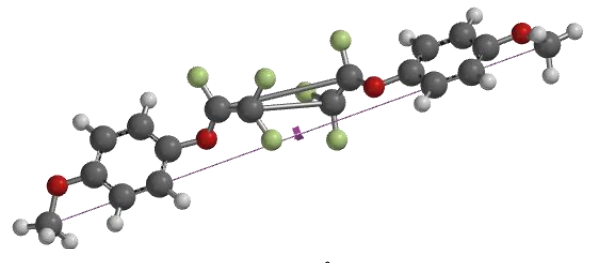

$18.972 \AA$ 


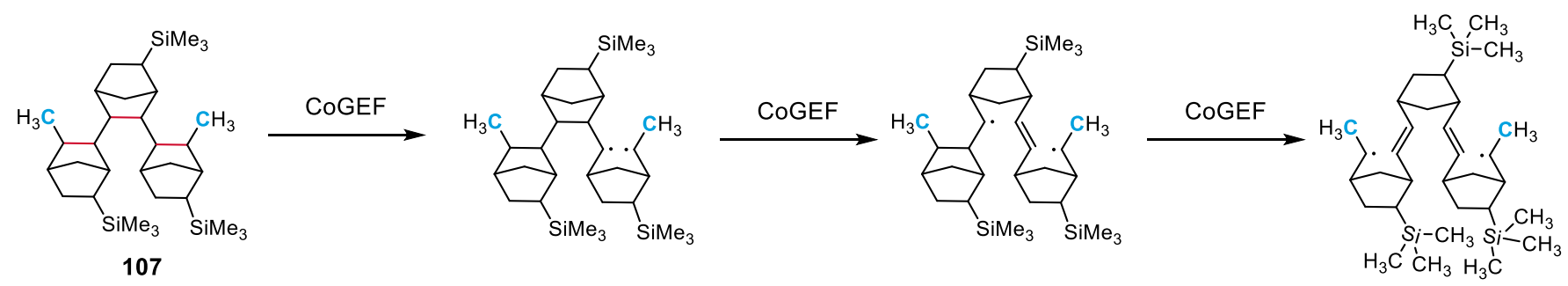

(i) Equilibrium Geometry

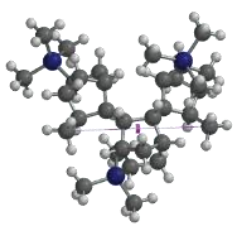

$8.084 \AA$ (ii) Immediately Prior to First Bond Cleavage

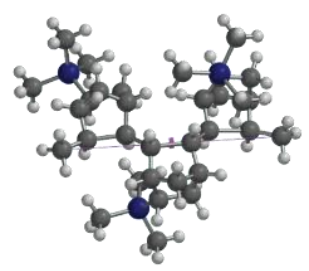

$10.534 \AA$

(vi) Immediately Prior to Third Bond Cleavage Bond Cleavage

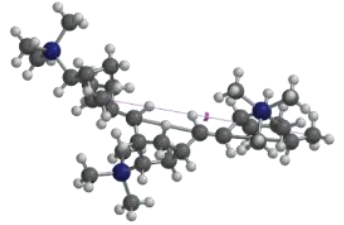

$13.284 \AA$

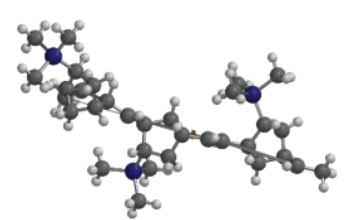

$17.734 \AA$ (iii) Immediately After First Bond Cleavage

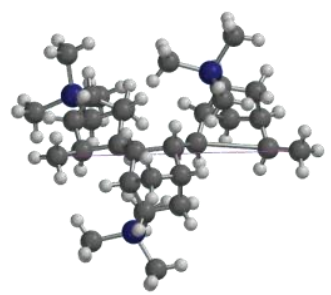

$10.584 \AA$

(vii) Immediately After Third Bond Cleavage

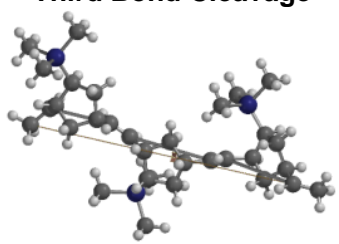

$17.784 \AA$ (iv) Immediately Prior to Second Bond Cleavage

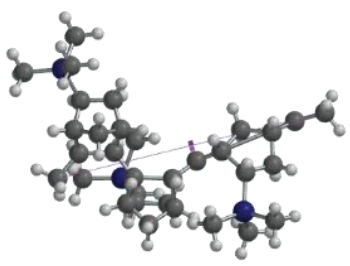

$13.234 \AA$

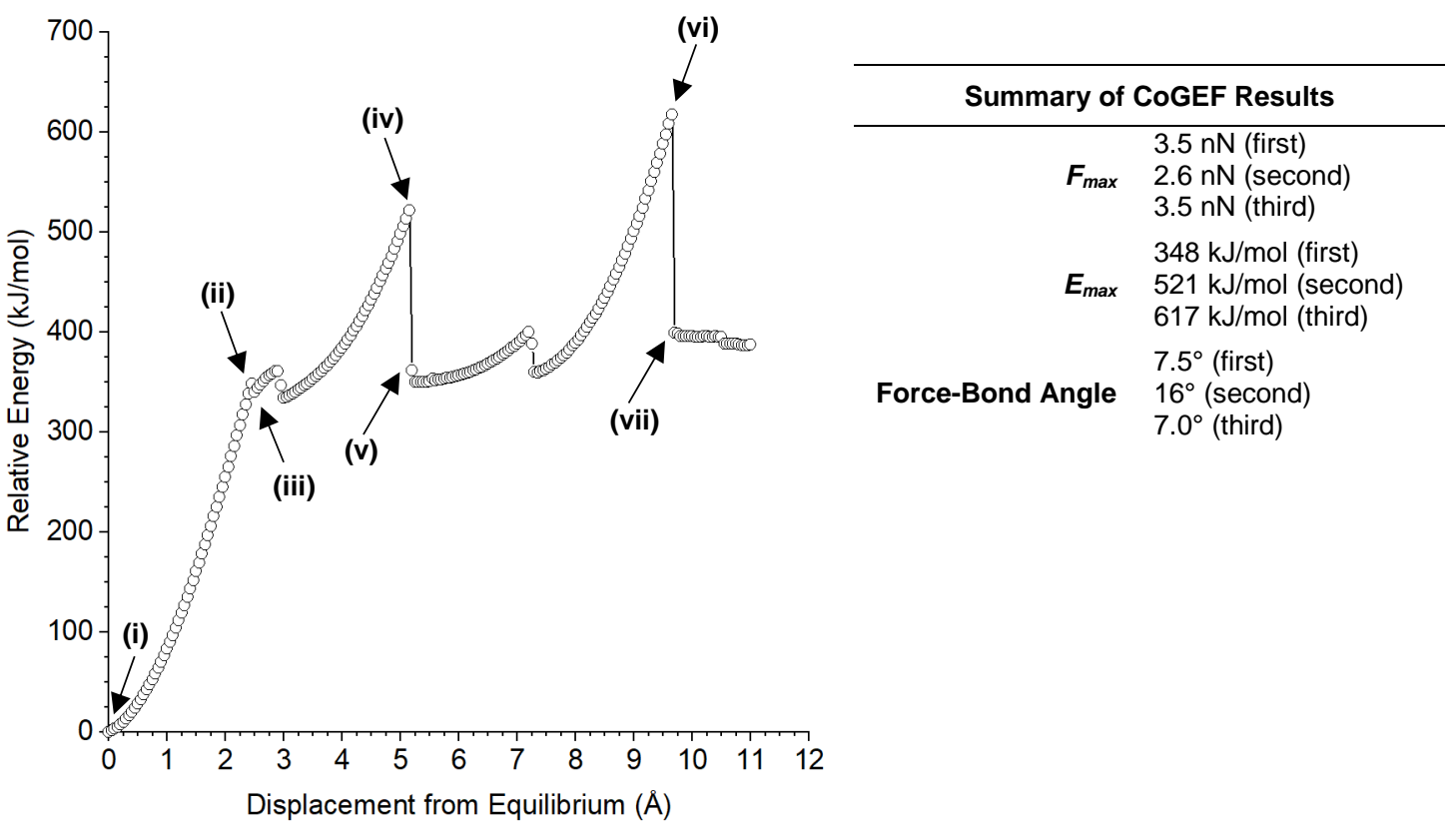


<smiles>CCOc1ccc(C2=NC(c3ccccc3)(c3ccccc3)N=C2c2ccccc2)c(-c2nc(-c3ccccc3)c(-c3ccccc3)n2C)c1</smiles><smiles>CCOc1ccc(C2N=C(c3ccccc3)C(c3ccccc3)=N2)cc1</smiles>

(ii)

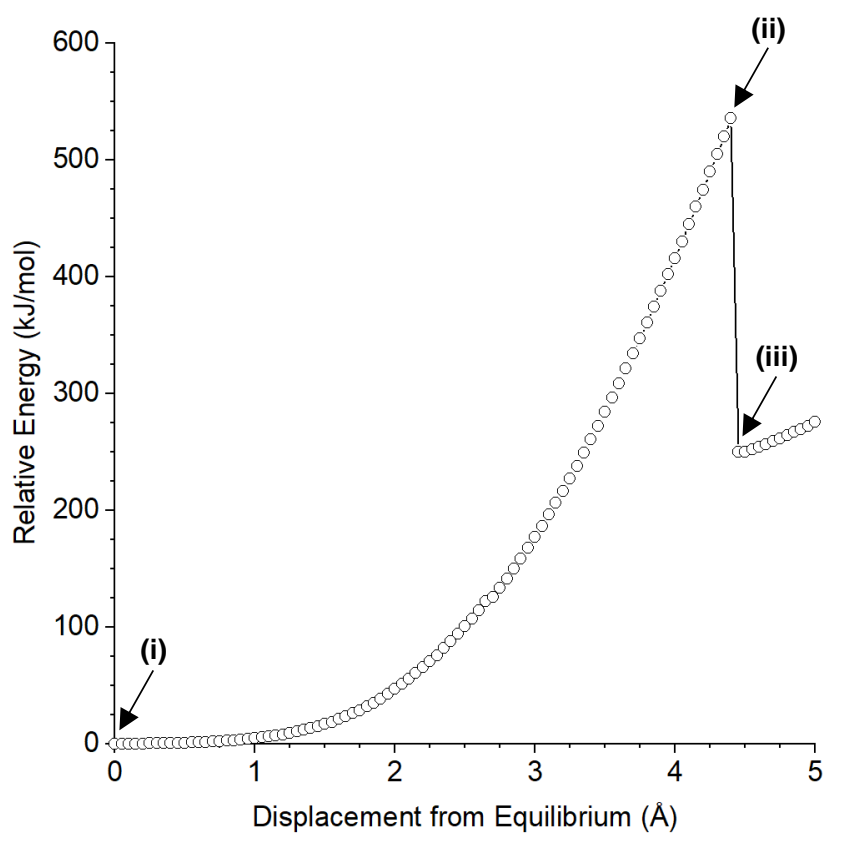

Summary of CoGEF Results

$\boldsymbol{F}_{\max } \quad 5.1 \mathrm{nN}$

$E_{\max } 536 \mathrm{~kJ} / \mathrm{mol}$

Force-Bond Angle $43^{\circ}$ (i) Equilibrium Geometry

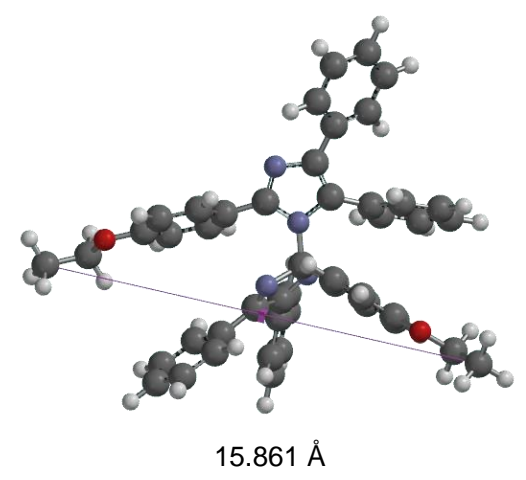

(ii) Immediately Prior to Bond Cleavage

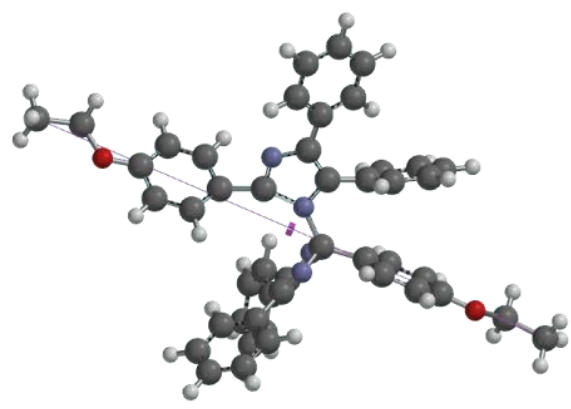

$20.261 \AA$

(iii) Immediately After Bond Cleavage

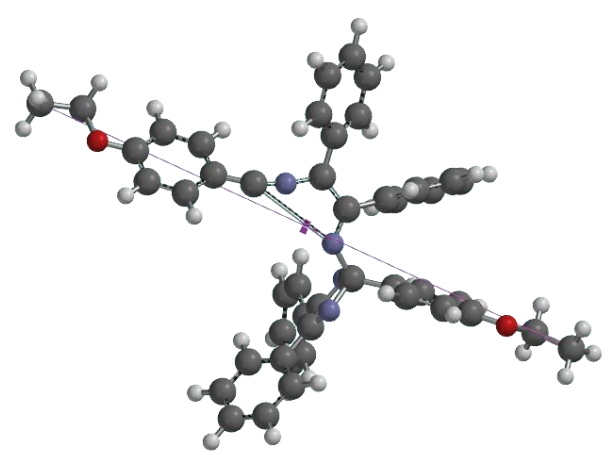

$20.311 \AA$ 


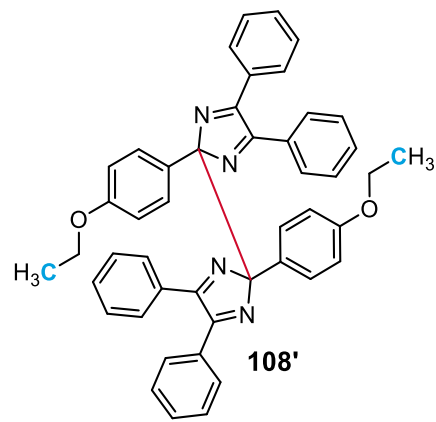

(ii)

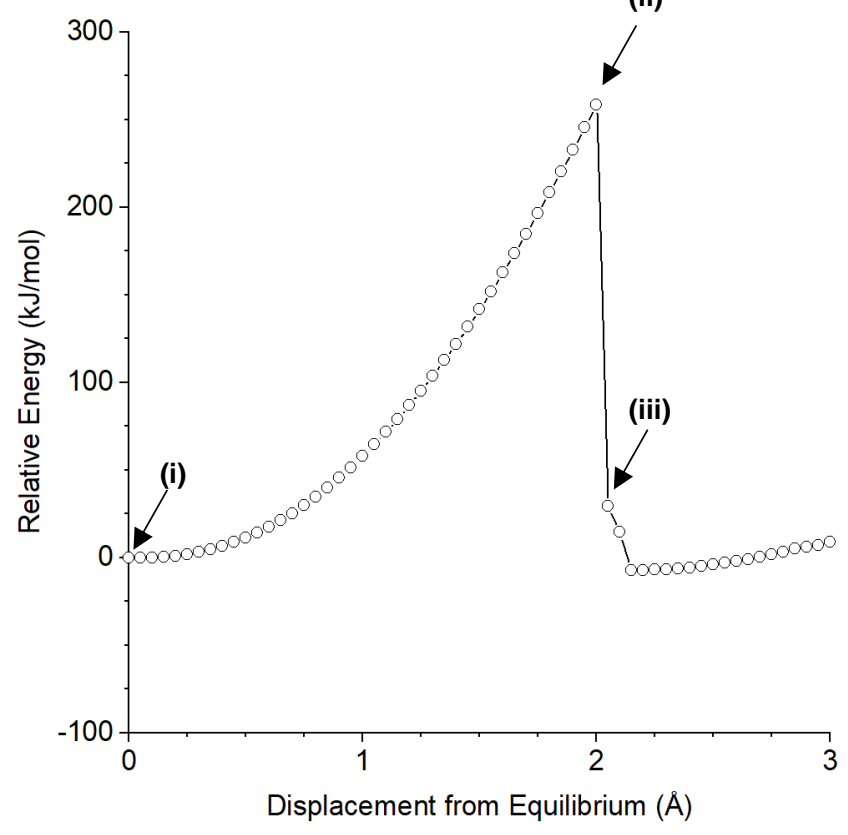

Summary of CoGEF Results

$F_{\max } \quad 4.3 \mathrm{nN}$

$E_{\max } 258 \mathrm{~kJ} / \mathrm{mol}$

Force-Bond Angle $40^{\circ}$

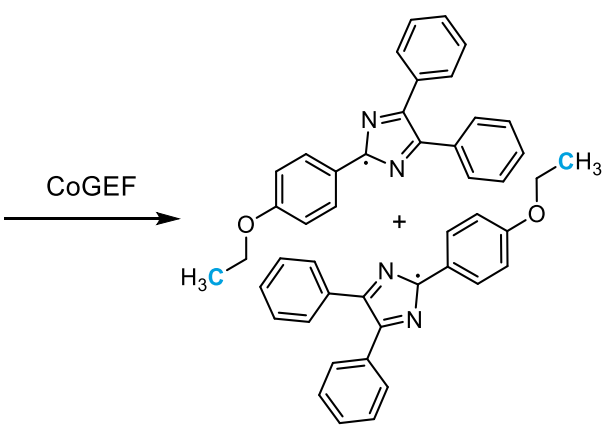

(i) Equilibrium Geometry

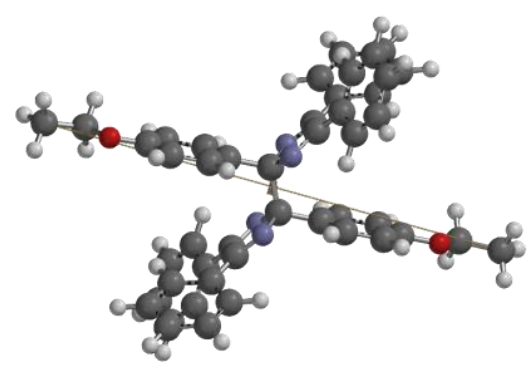

$16.537 \AA$

(ii) Immediately Prior to Bond Cleavage

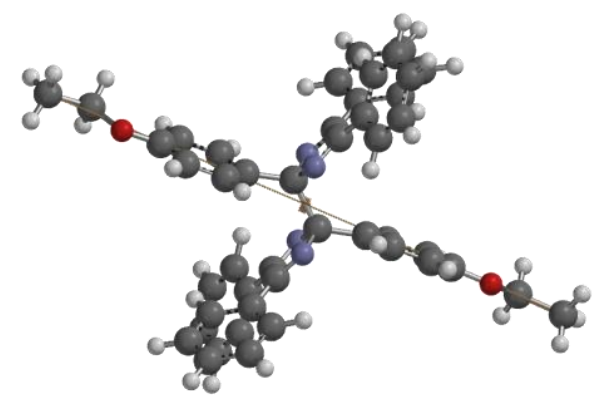

$18.537 \AA$

(iii) Immediately After Bond Cleavage

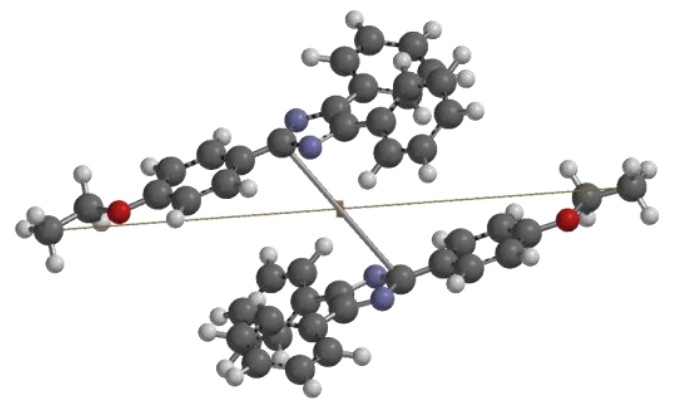

$18.587 \AA$ 

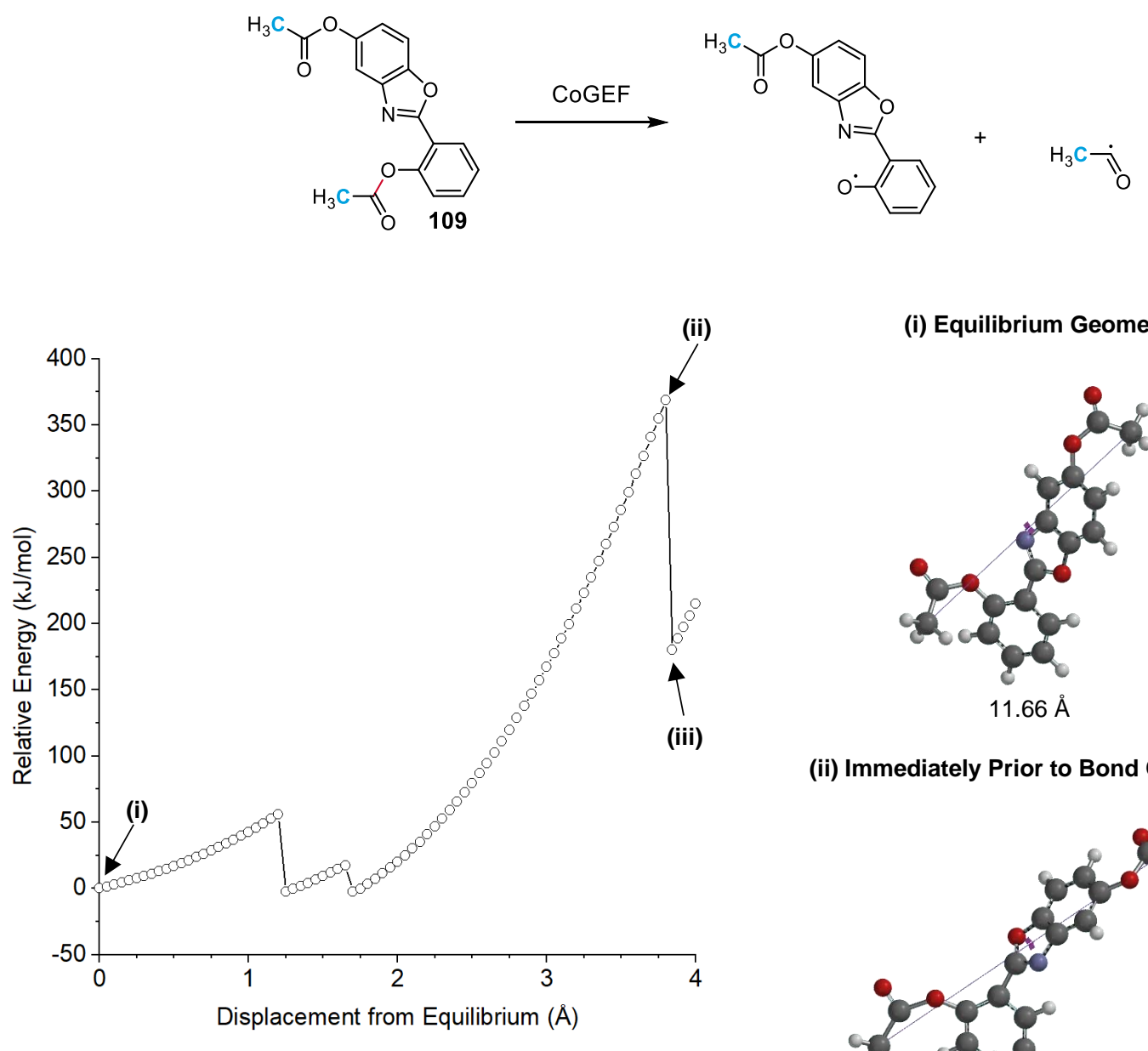

(i) Equilibrium Geometry

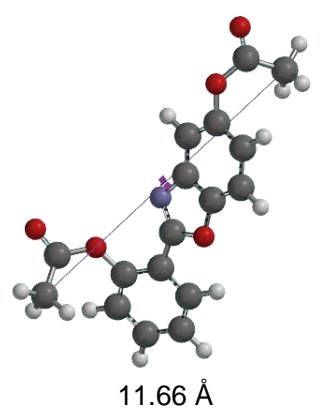

(ii) Immediately Prior to Bond Cleavage

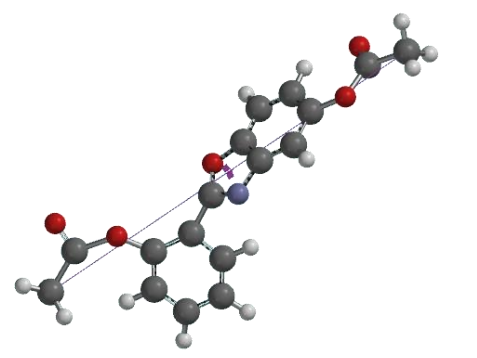

$15.41 \AA$

Summary of CoGEF Results

(iii) Immediately After Bond Cleavage

$\boldsymbol{F}_{\max } \quad 4.7 \mathrm{nN}$

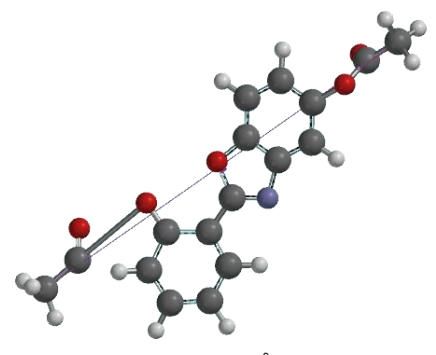

$15.46 \AA$ 


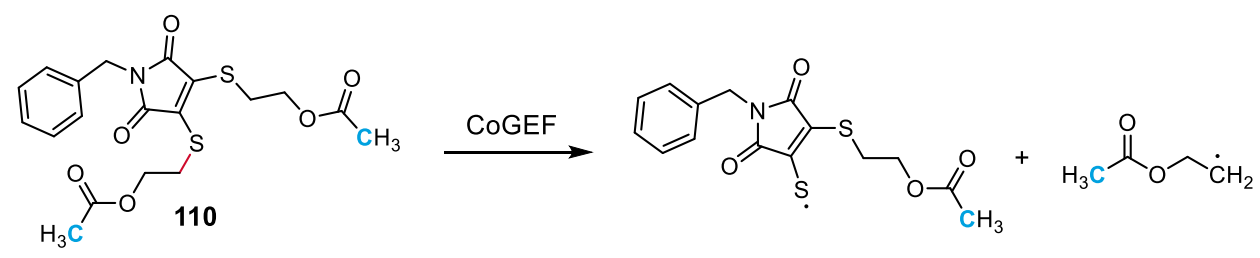

(ii)

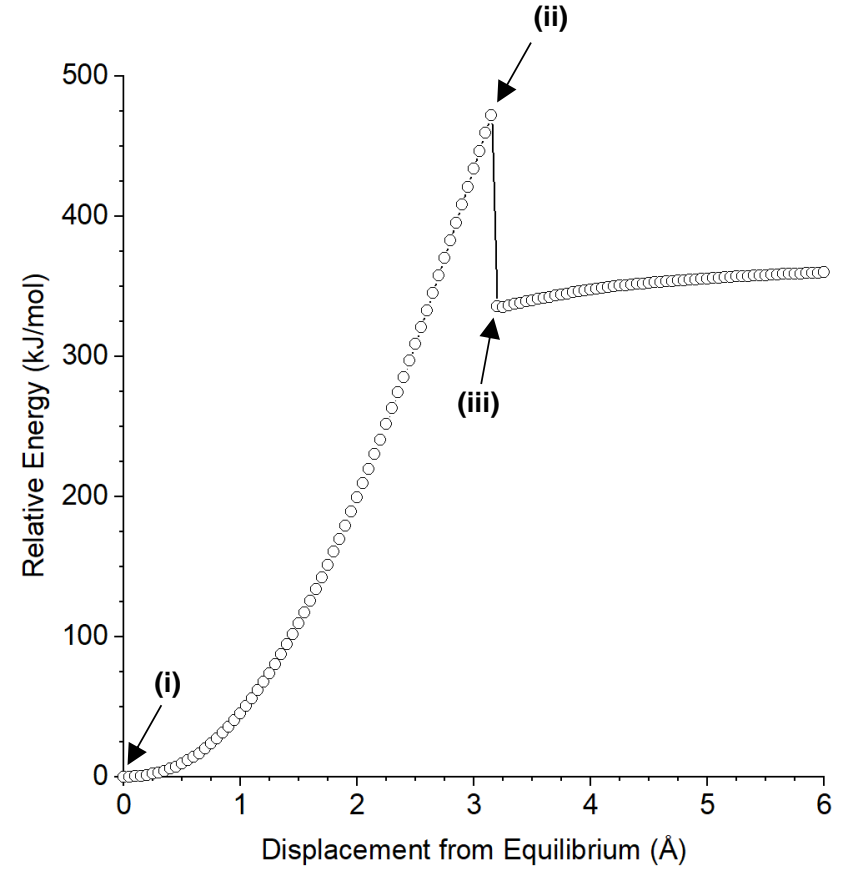

Summary of CoGEF Results

$F_{\text {max }} \quad 4.3 \mathrm{nN}$

$E_{\max } \quad 472 \mathrm{~kJ} / \mathrm{mol}$

Force-Bond Angle $24^{\circ}$ (i) Equilibrium Geometry

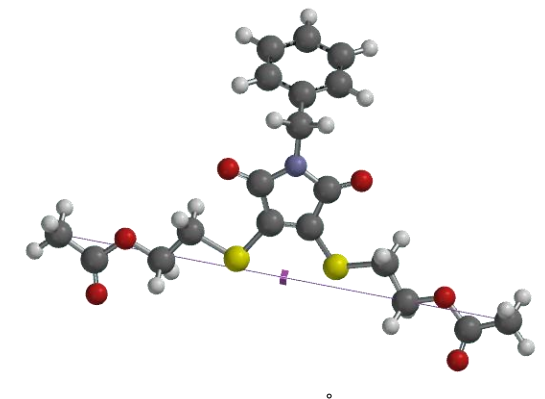

$15.926 \AA$

(ii) Immediately Prior to Bond Cleavage

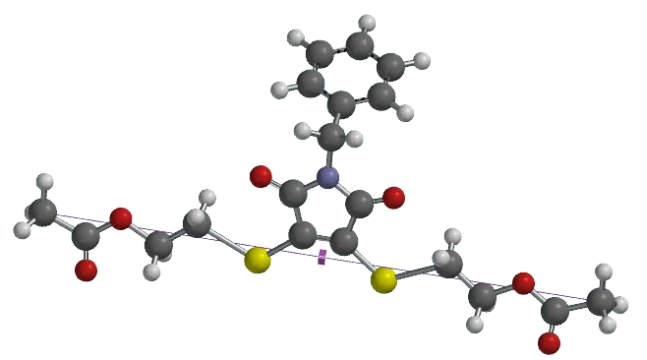

$19.076 \AA$

(iii) Immediately After Bond Cleavage

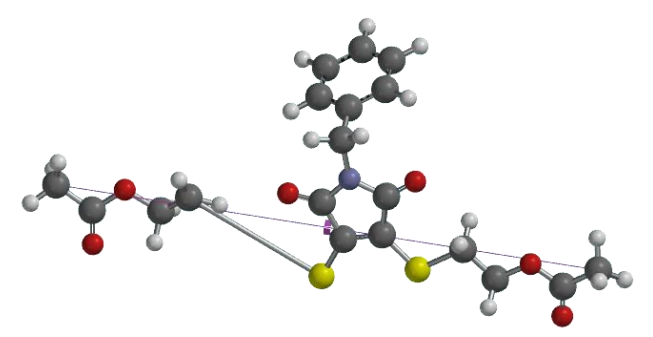

$19.126 \AA$ 


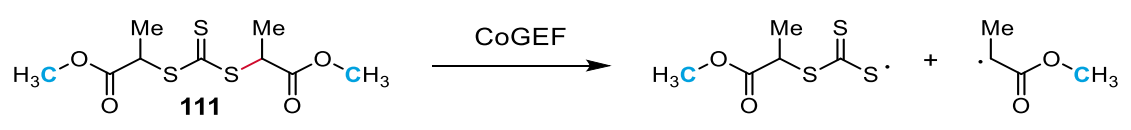

(i) Equilibrium Geometry
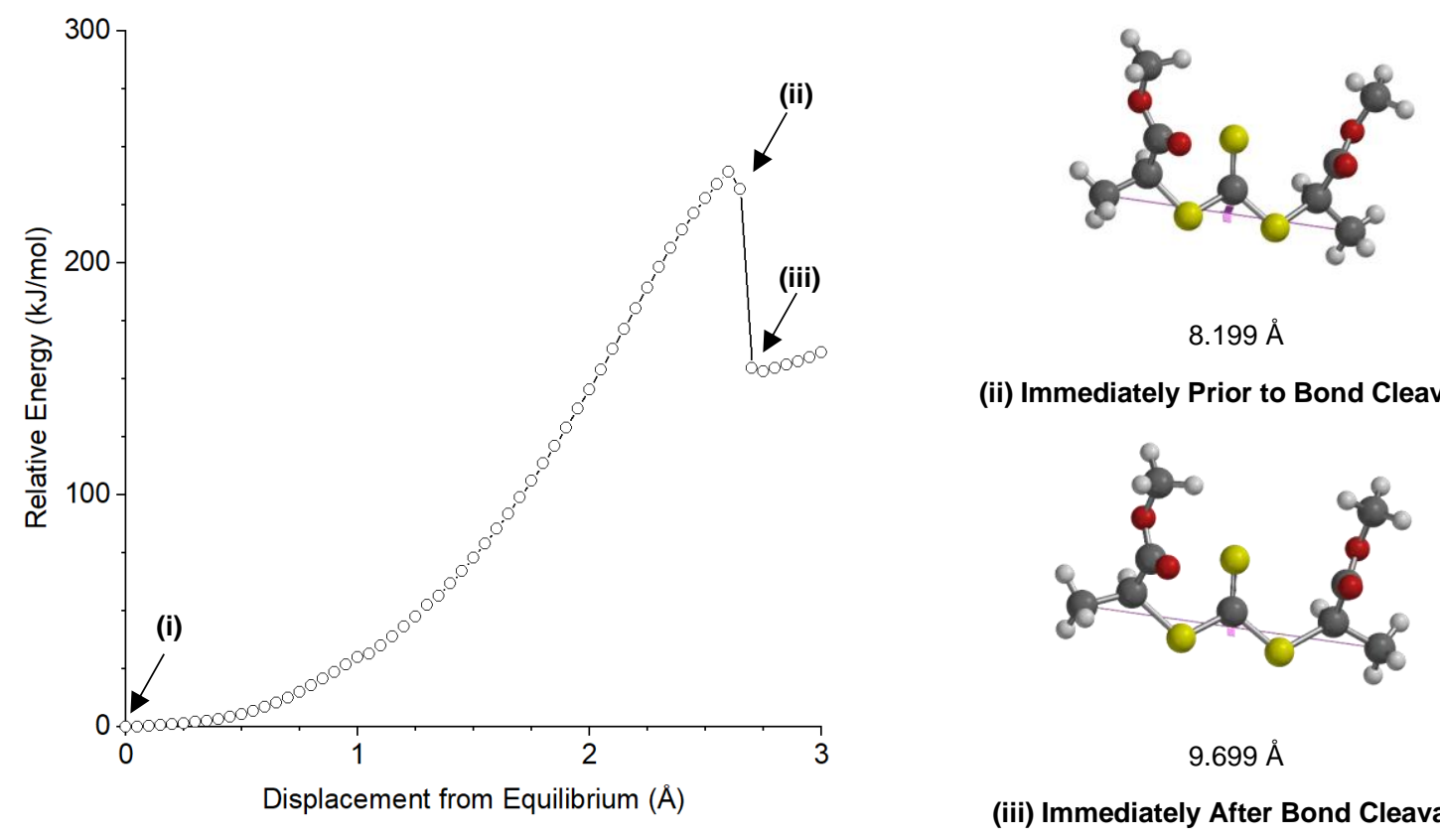

$8.199 \AA$

(ii) Immediately Prior to Bond Cleavage

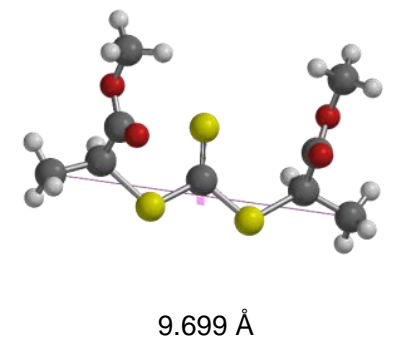

(iii) Immediately After Bond Cleavage

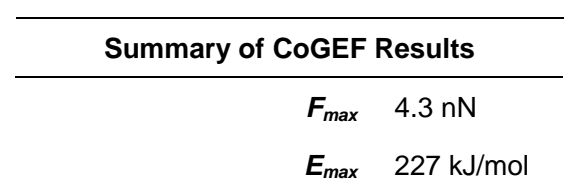

Force-Bond Angle $35^{\circ}$

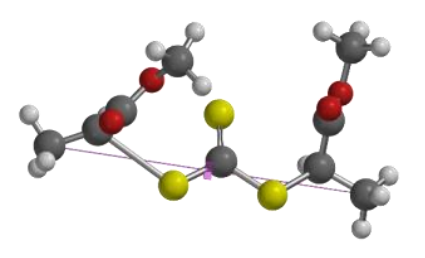

$9.749 \AA$ 


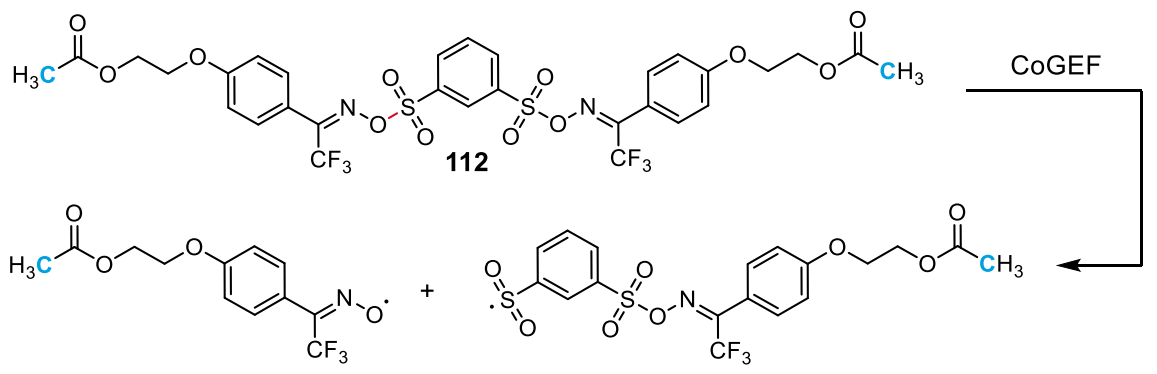

(i) Equilibrium Geometry

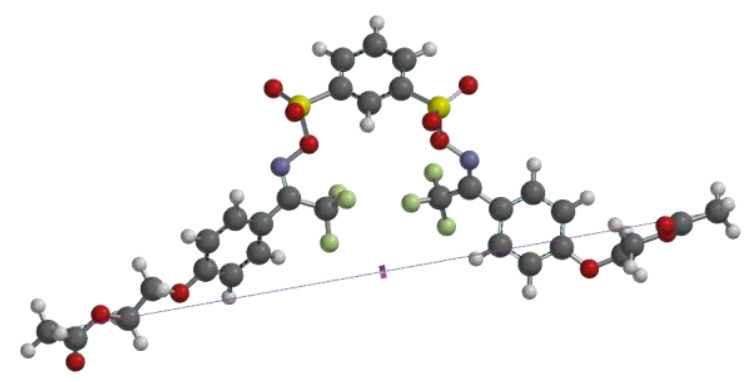

$26.482 \AA$

(ii) Immediately Prior to Bond Cleavage

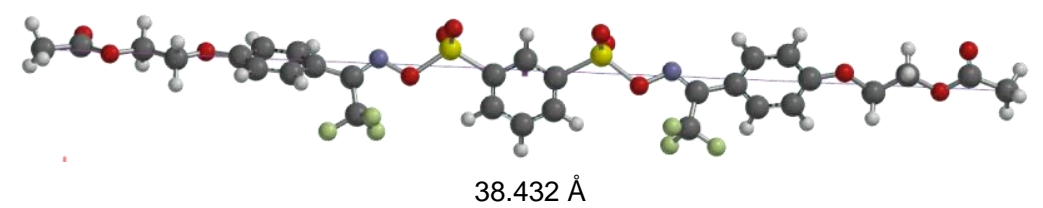

(iii) Immediately After Bond Cleavage

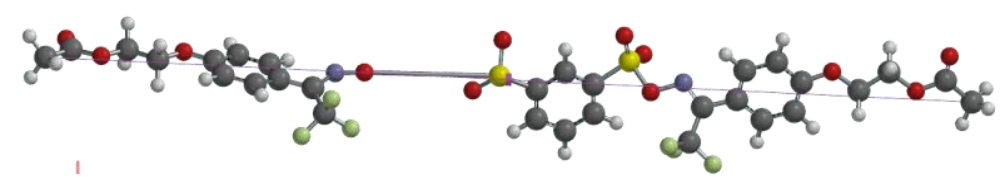

$38.482 \AA$

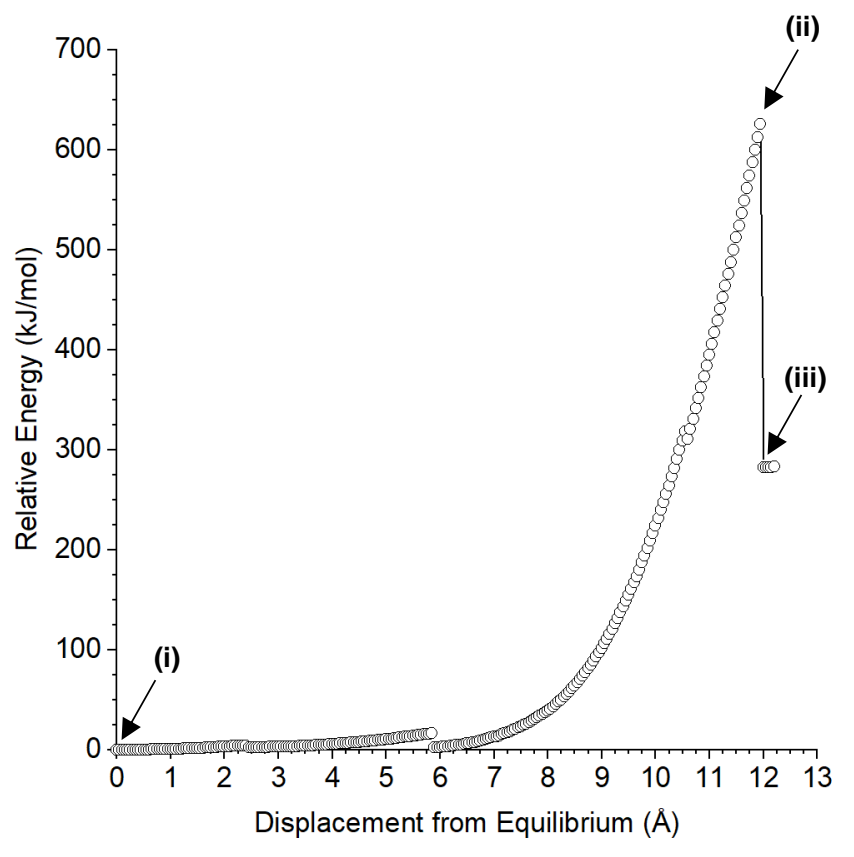

Summary of CoGEF Results

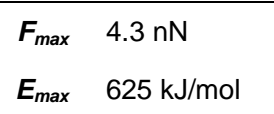

Force-Bond Angle $28^{\circ}$ 

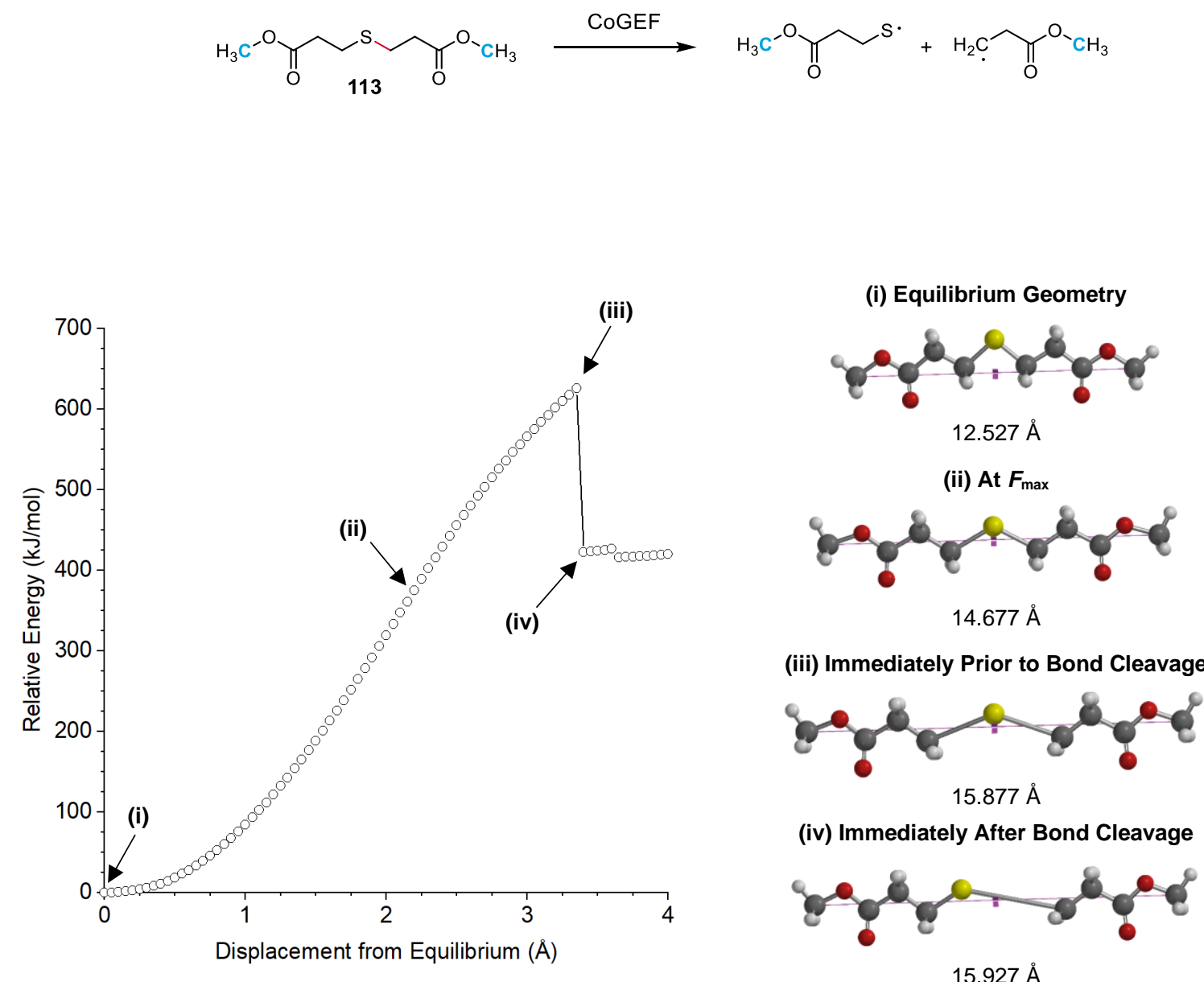

(iii) Immediately Prior to Bond Cleavage

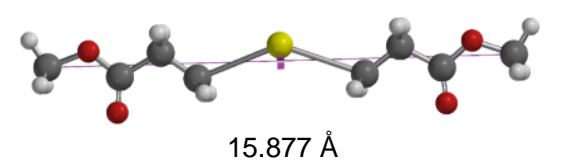

(iv) Immediately After Bond Cleavage
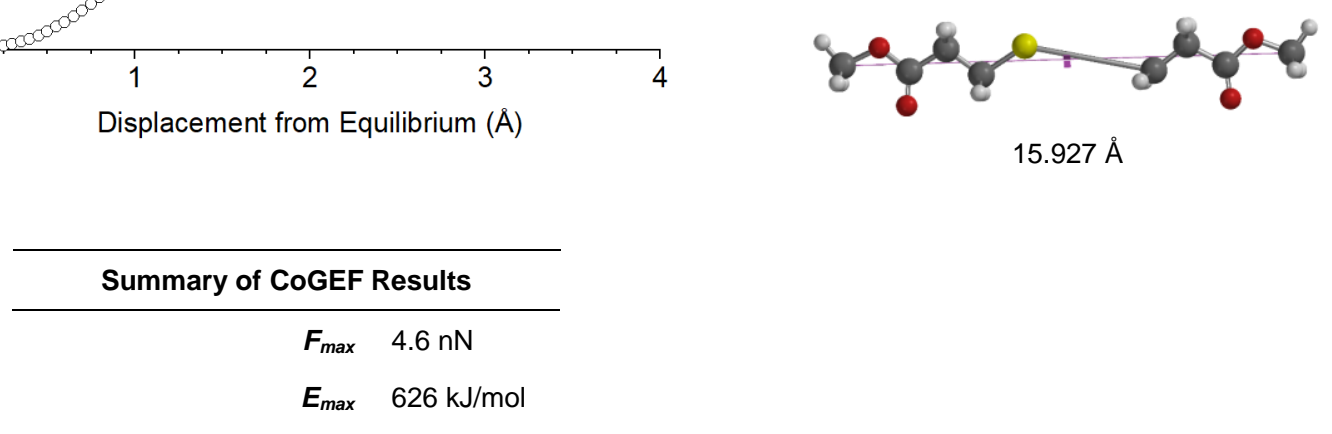

Force-Bond Angle $20^{\circ}$ 
<smiles>[CH2+]c1ccc(C(=O)OC)cc1</smiles>

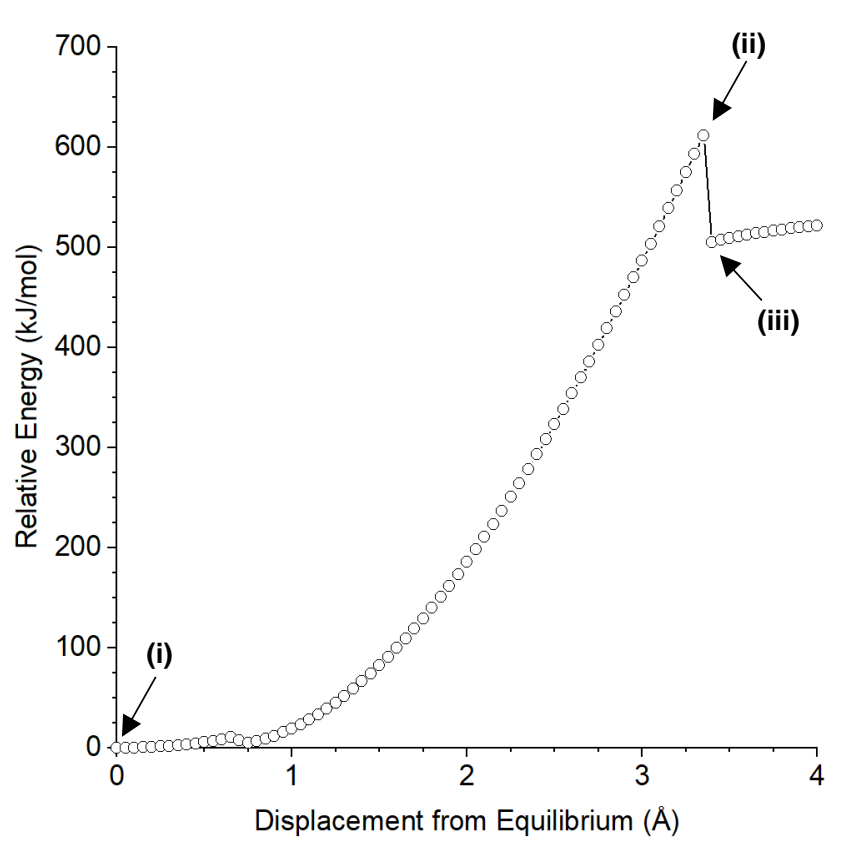

(i) Equilibrium Geometry

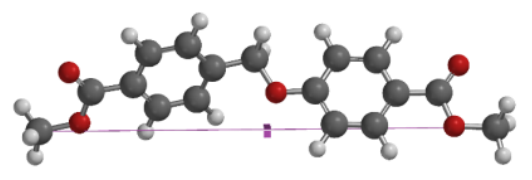

$15.877 \AA$

(ii) Immediately Prior to Bond Cleavage

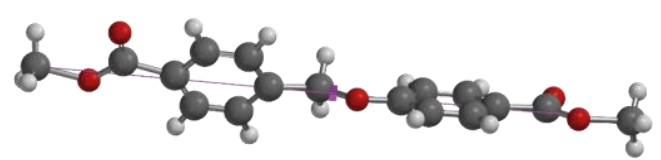

$19.227 \AA$

(iii) Immediately After Bond Cleavage

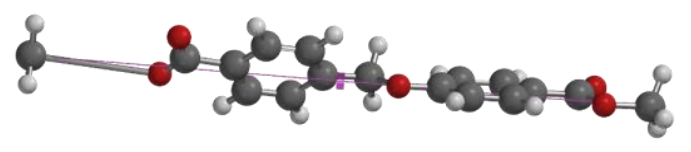

$19.277 \AA$

Summary of CoGEF Results

$$
\begin{array}{ll}
\boldsymbol{F}_{\max } & 6.1 \mathrm{nN} \\
\boldsymbol{E}_{\max } & 611 \mathrm{~kJ} / \mathrm{mol}
\end{array}
$$

Force-Bond Angle $37^{\circ}$ 


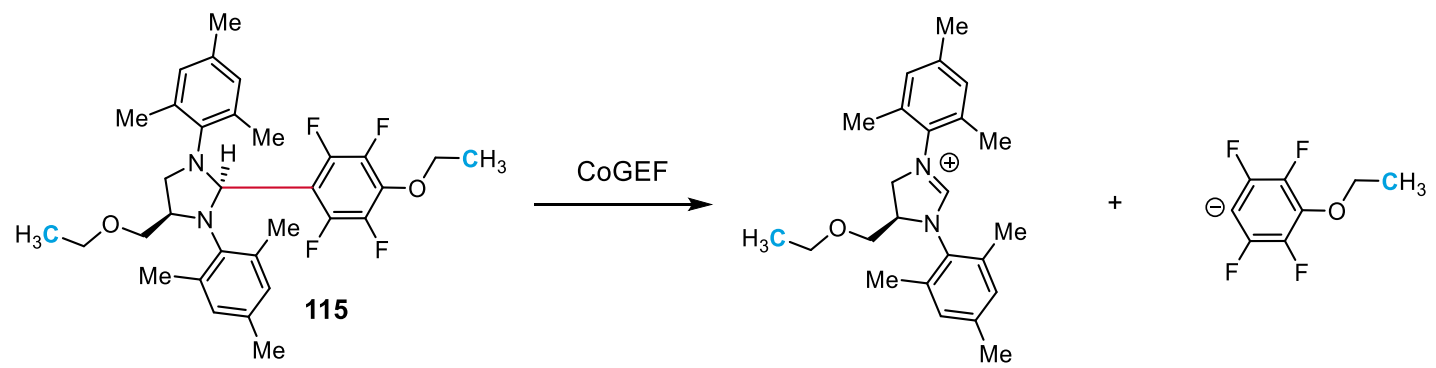

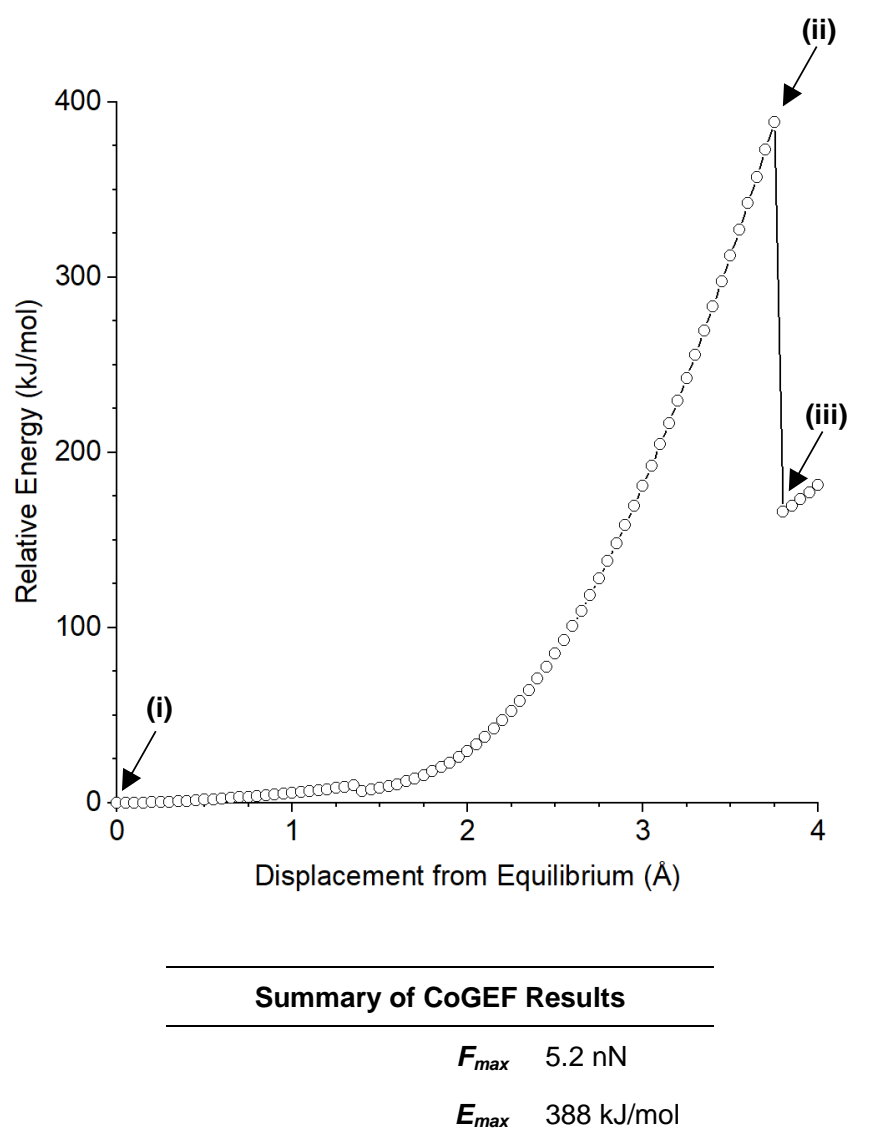

Force-Bond Angle $13^{\circ}$
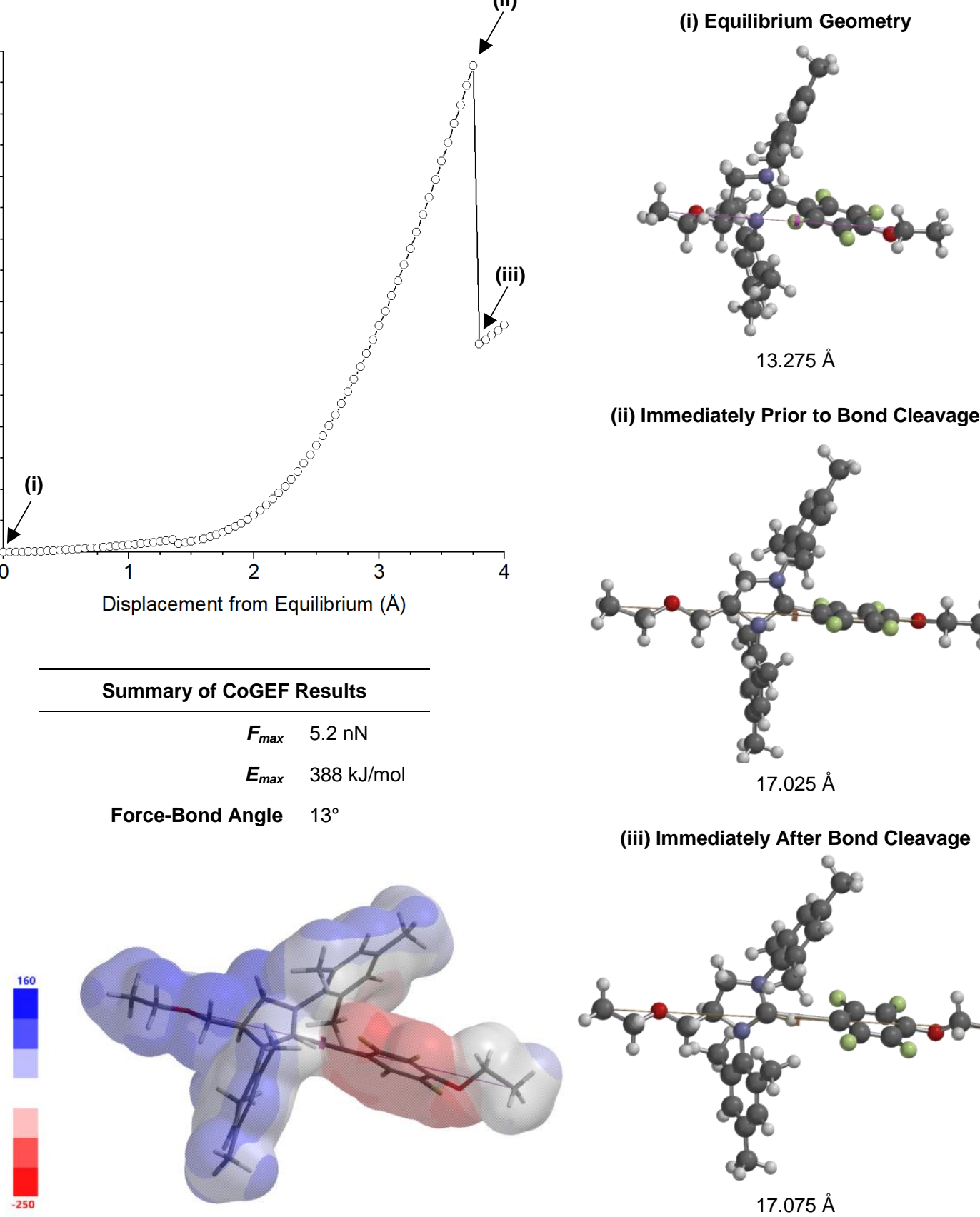

(ii) Immediately Prior to Bond Cleavage

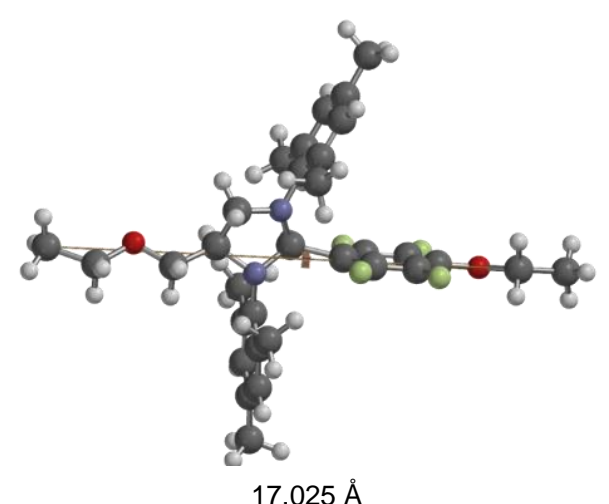

(iii) Immediately After Bond Cleavage

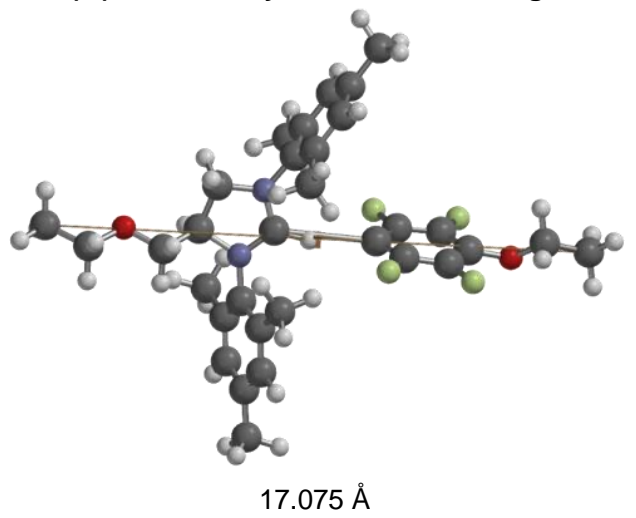



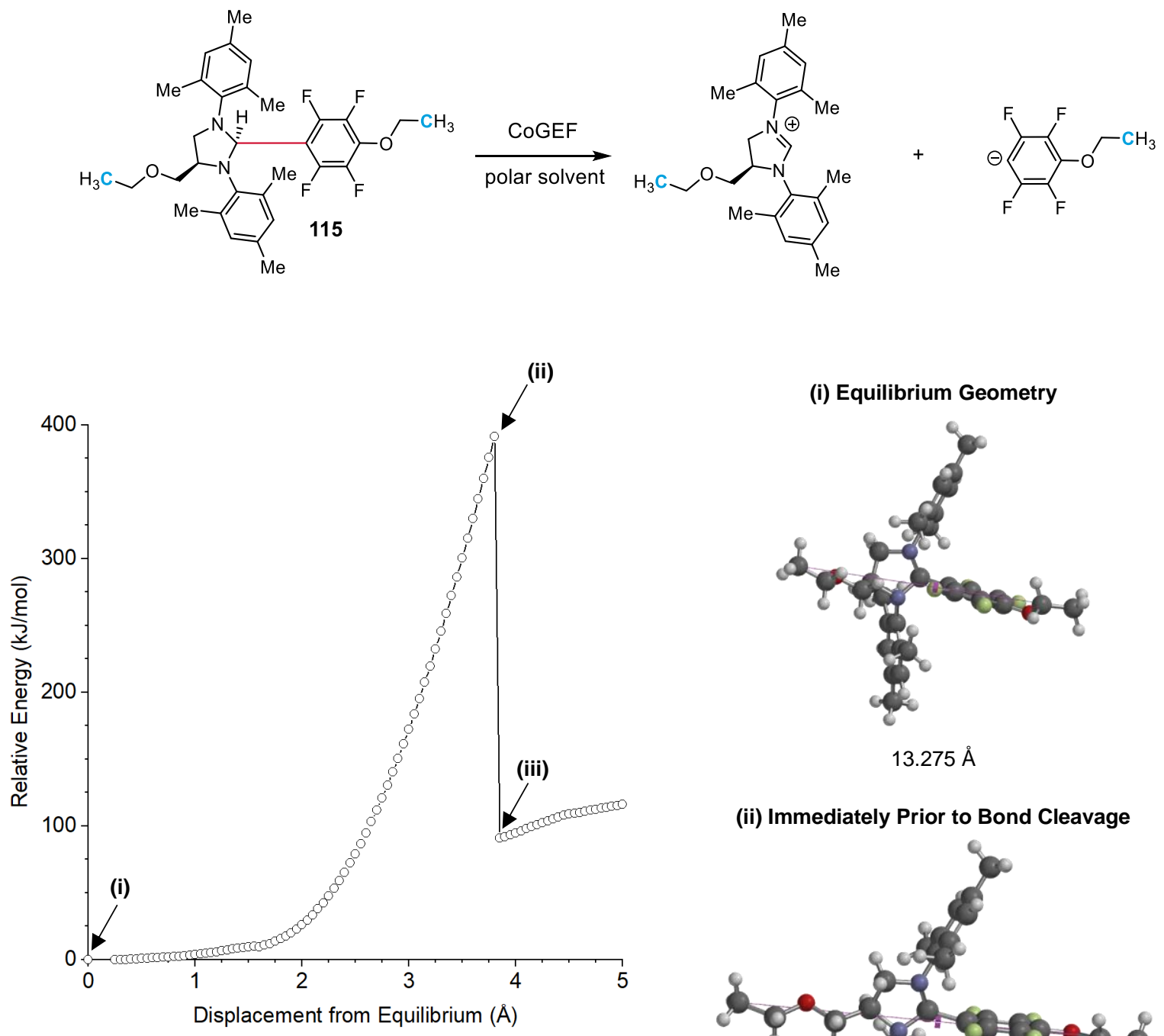

(ii) Immediately Prior to Bond Cleavage

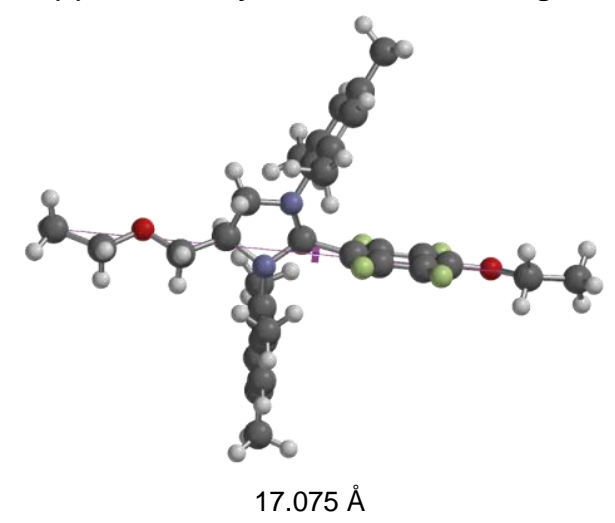

(iii) Immediately After Bond Cleavage

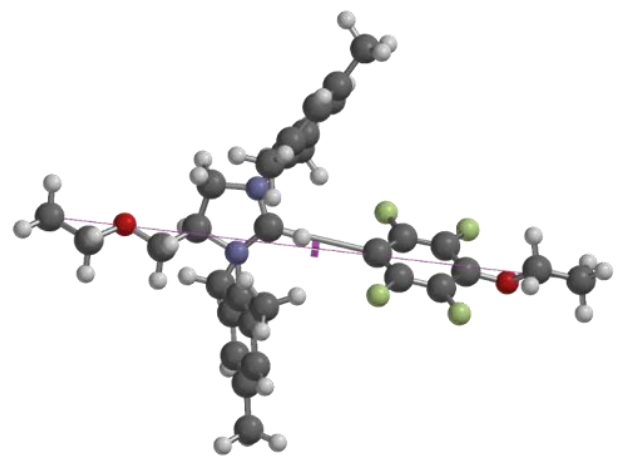

$17.125 \AA$ 


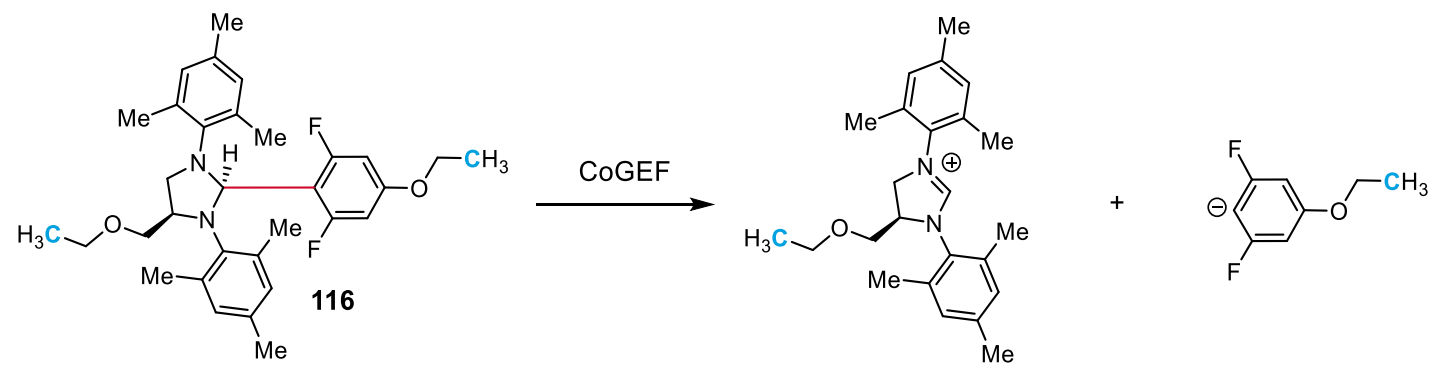

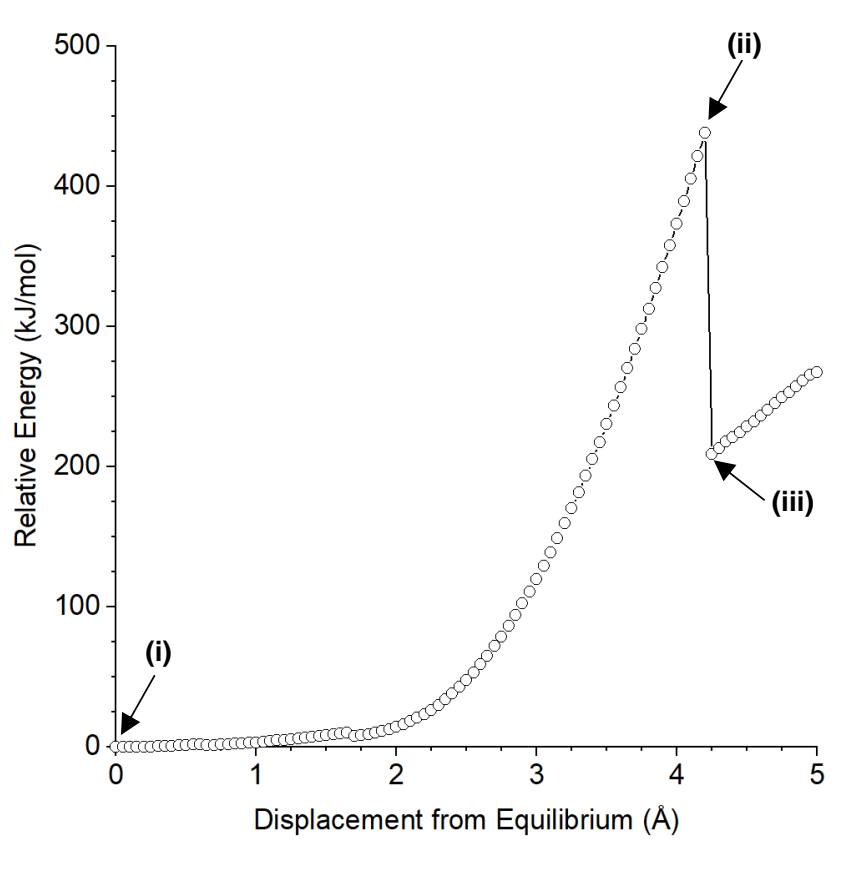

(i) Equilibrium Geometry

Summary of CoGEF Results

$$
\begin{array}{ll}
F_{\max } & 5.5 \mathrm{nN} \\
E_{\max } & 438 \mathrm{~kJ} / \mathrm{mol}
\end{array}
$$

Force-Bond Angle $13^{\circ}$
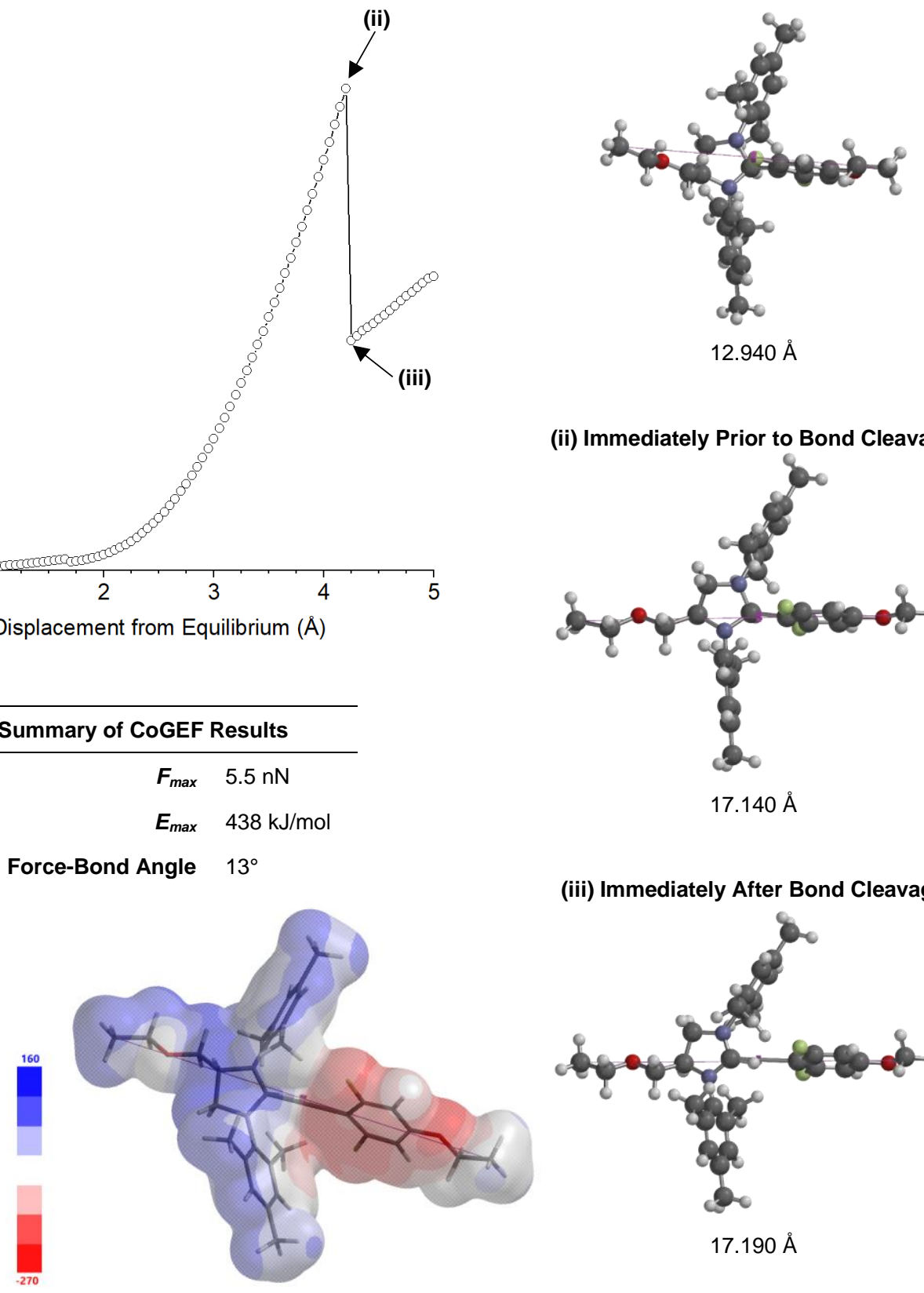

(ii) Immediately Prior to Bond Cleavage

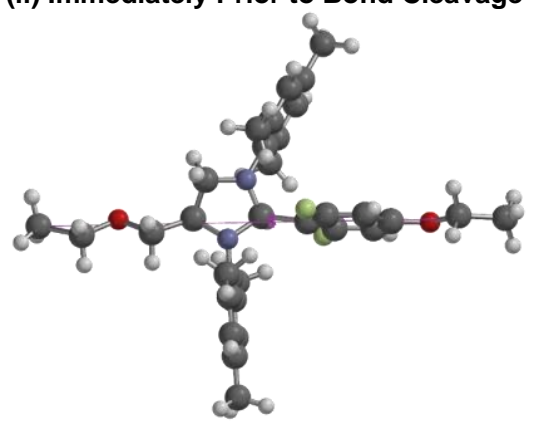

$17.140 \AA$

(iii) Immediately After Bond Cleavage

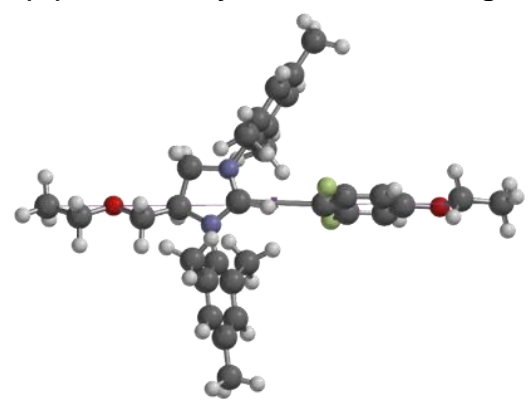

$17.190 \AA$ 
<smiles>CCOCC1CN(c2c([N+](=O)[O-])cc([N+](=O)[O-])cc2[N+](=O)[O-])C(c2c([N+](=O)[O-])cc([N+](=O)[O-])cc2[N+](=O)[O-])CN1c1c([N+](=O)[O-])cc([N+](=O)[O-])cc1[N+](=O)[O-]</smiles>

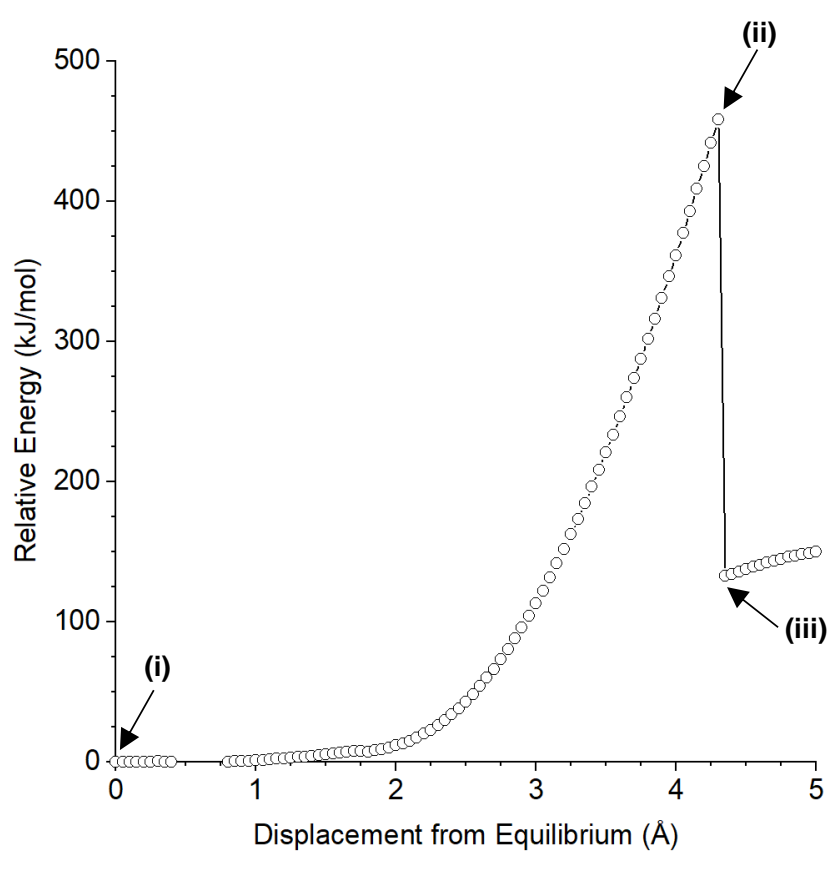

(i) Equilibrium Geometry

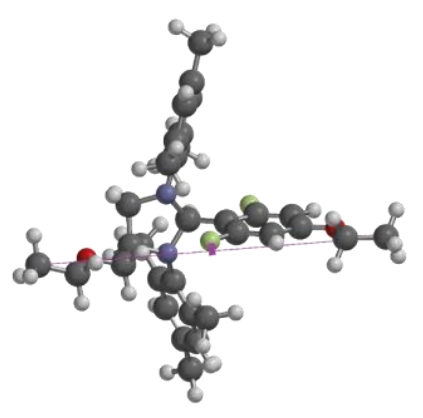

$12.940 \AA$

(ii) Immediately Prior to Bond Cleavage
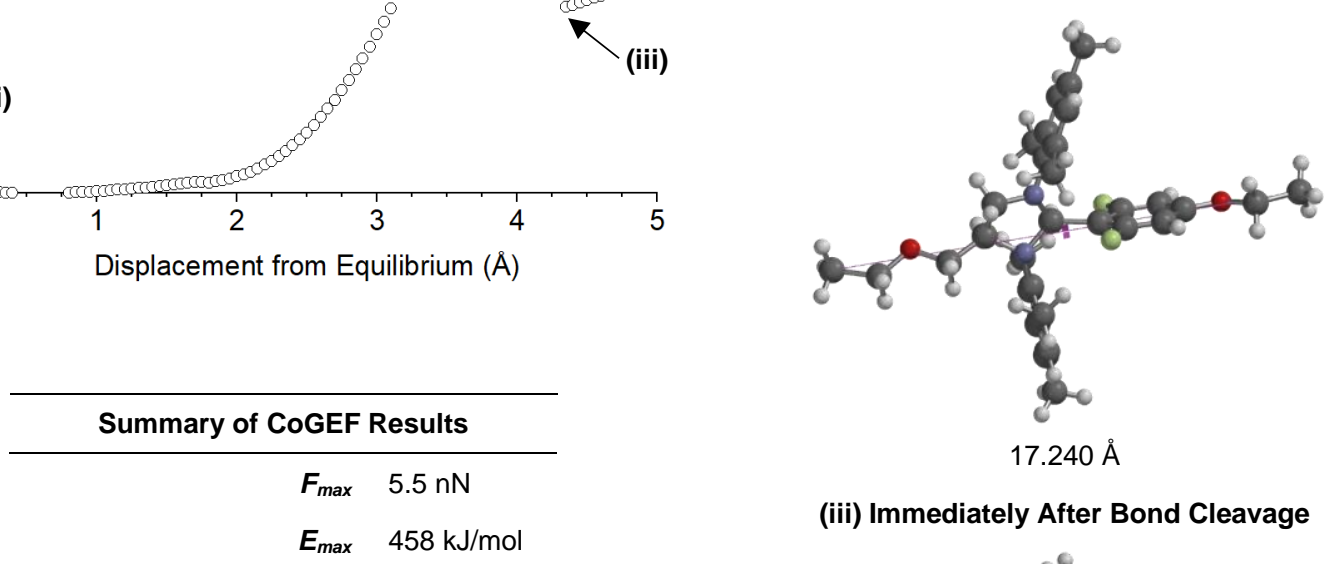

$17.240 \AA$

(iii) Immediately After Bond Cleavage

Force-Bond Angle $12^{\circ}$

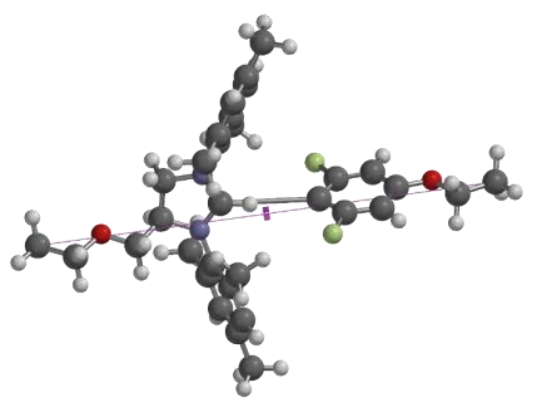

$17.290 \AA$ 
<smiles>CCOCC1CN(c2c([N+](=O)[O-])cc([N+](=O)[O-])cc2[N+](=O)[O-])C(COCC)N1c1c([N+](=O)[O-])cc([N+](=O)[O-])cc1[N+](=O)[O-]</smiles>
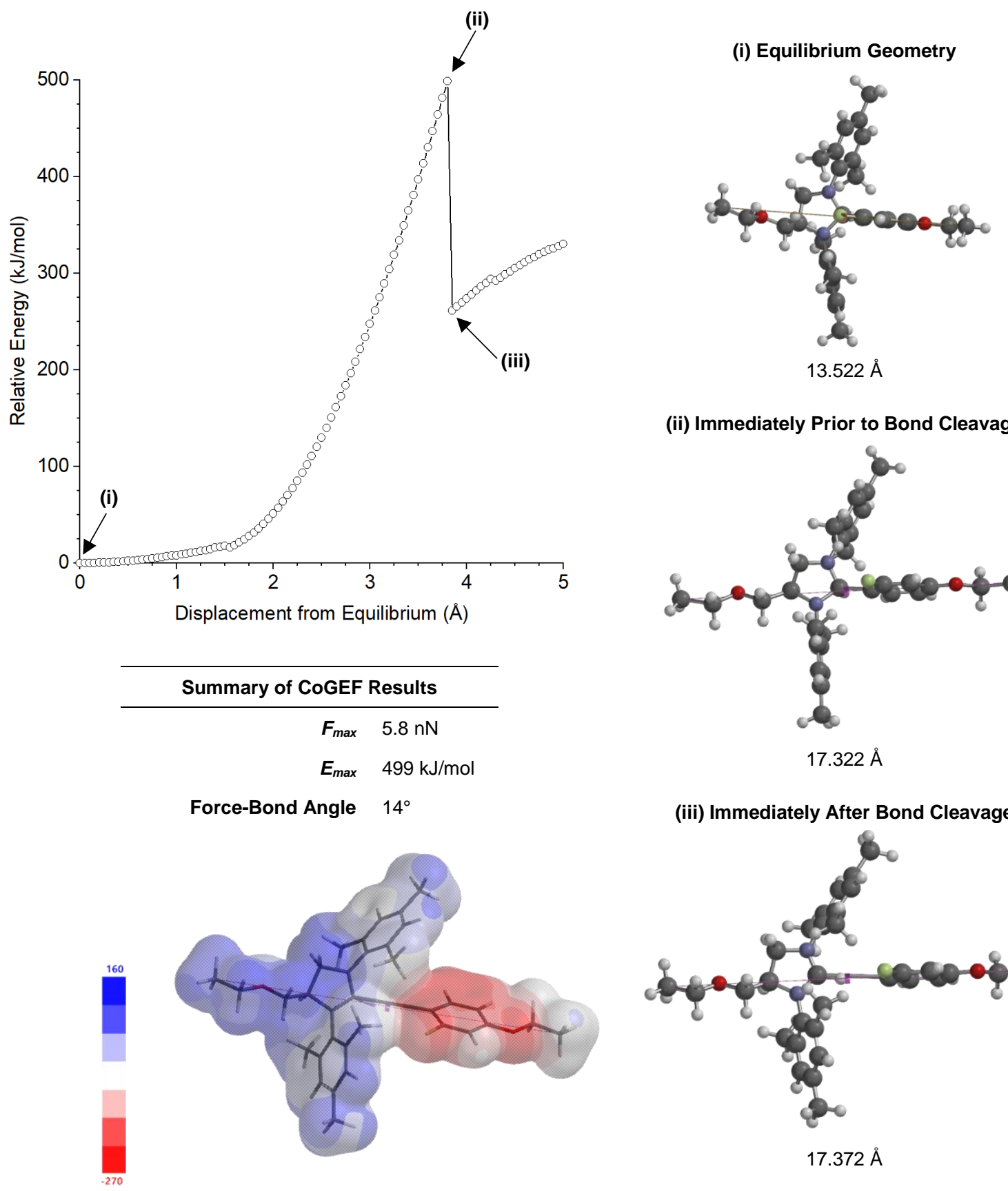

(ii) Immediately Prior to Bond Cleavage

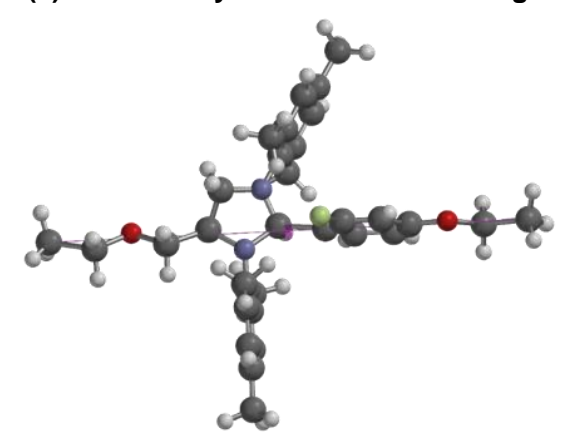

$17.322 \AA$

(iii) Immediately After Bond Cleavage

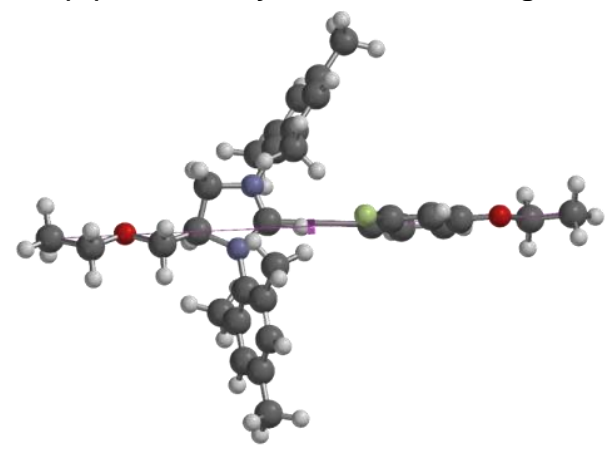

$17.372 \AA$ 

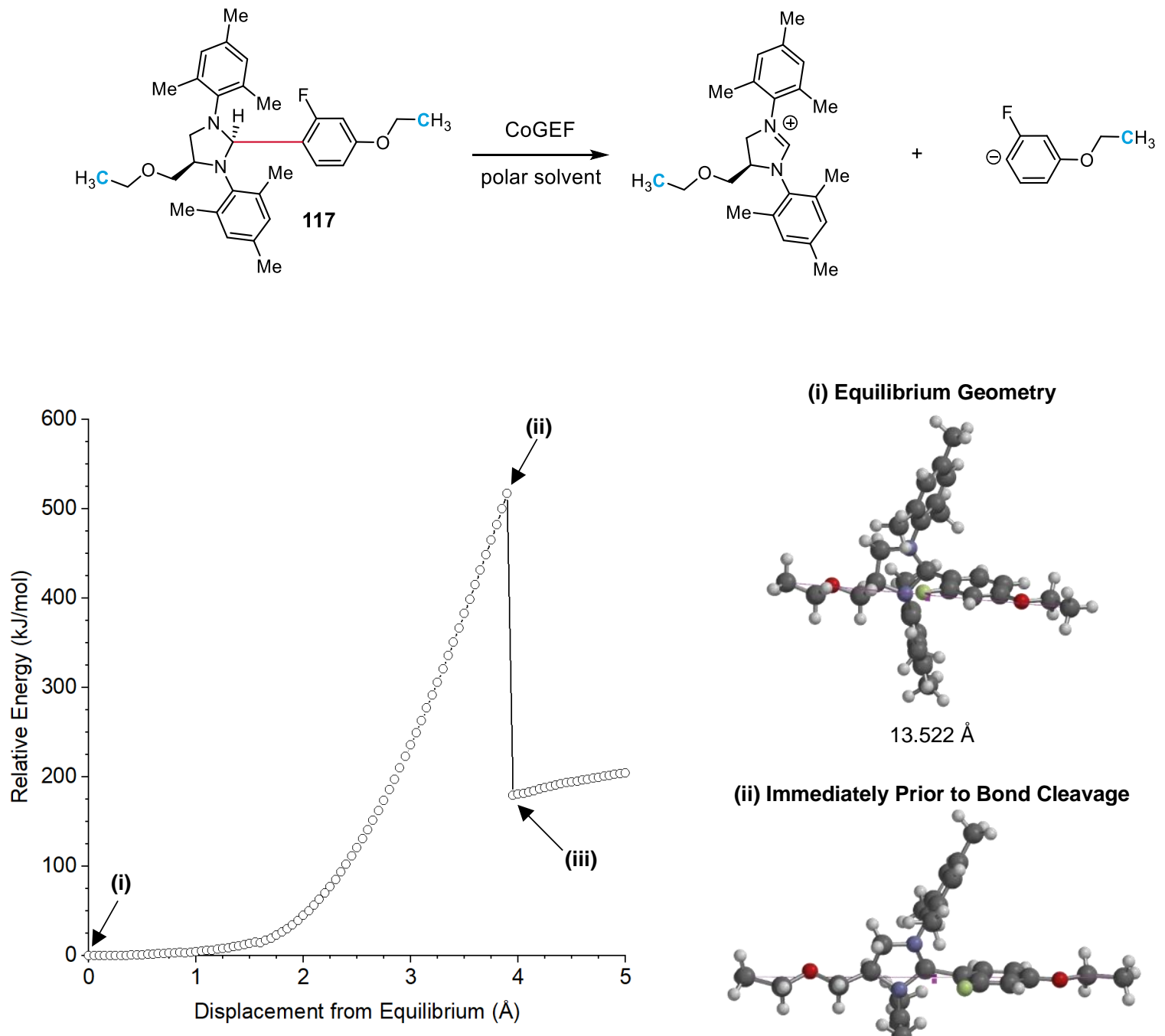

(i) Equilibrium Geometry

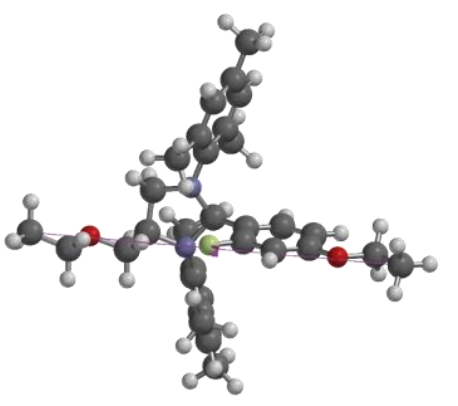

$13.522 \AA$

(ii) Immediately Prior to Bond Cleavage

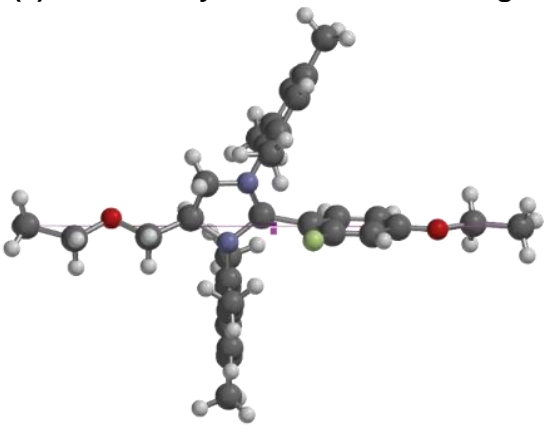

$17.422 \AA$

(iii) Immediately After Bond Cleavage

Force-Bond Angle $14^{\circ}$

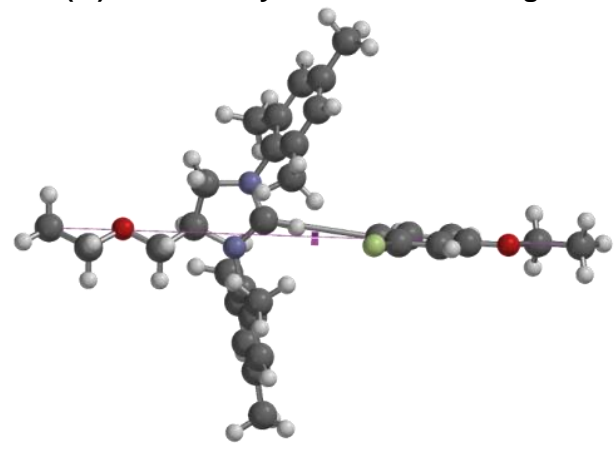

$17.472 \AA$ 

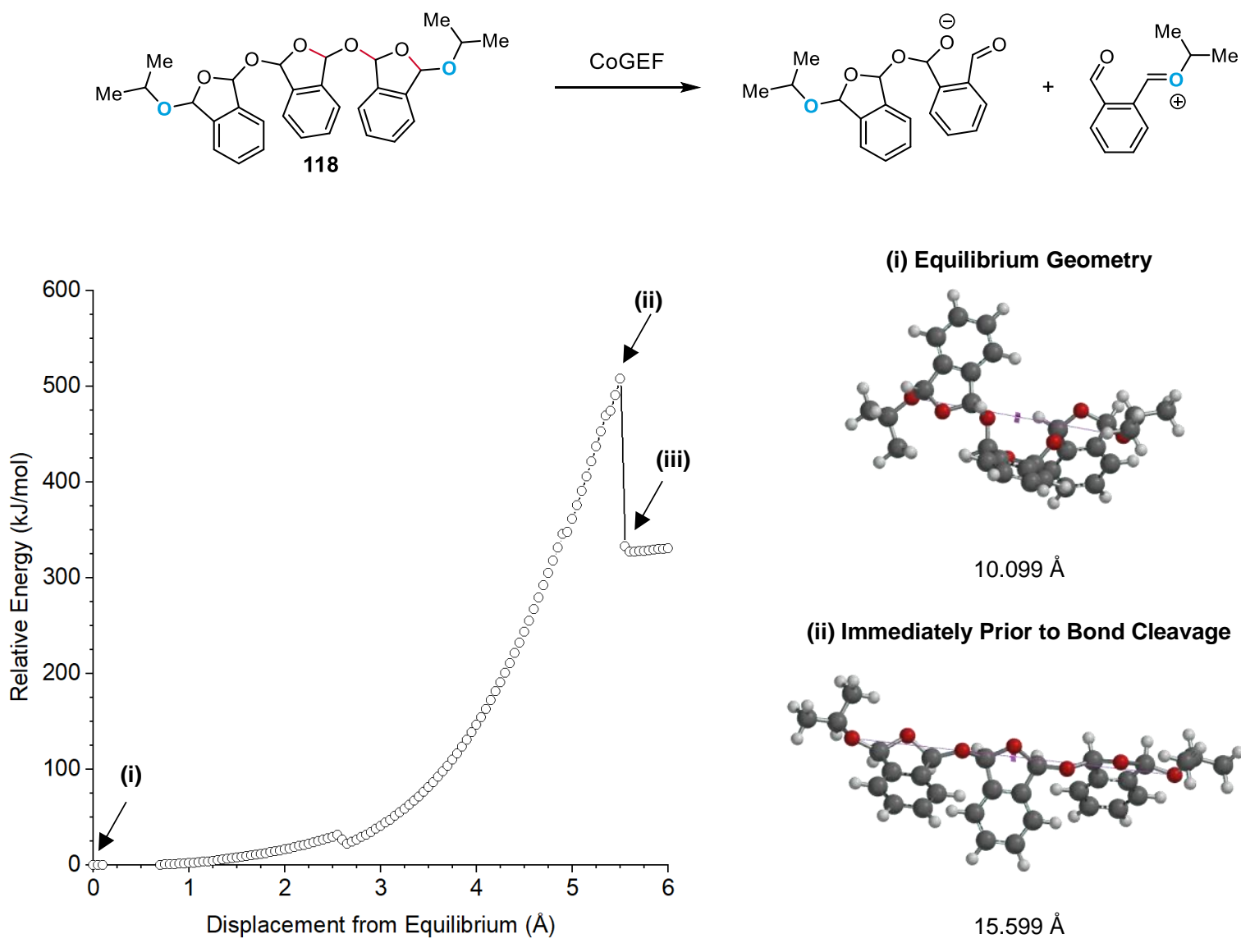

(i) Equilibrium Geometry

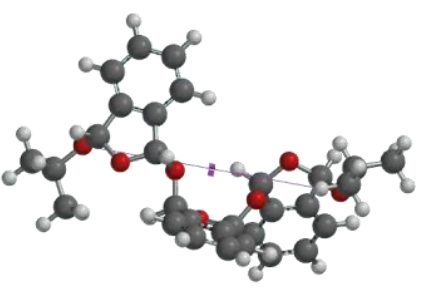

$10.099 \AA$

(ii) Immediately Prior to Bond Cleavage

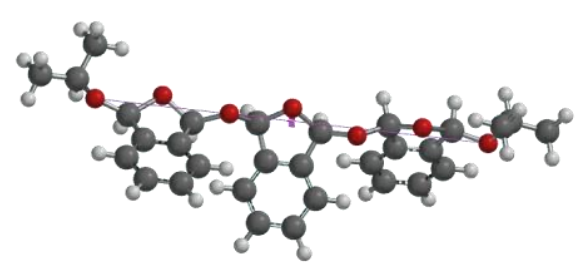

$15.599 \AA$

(iii) Immediately After Bond Cleavage

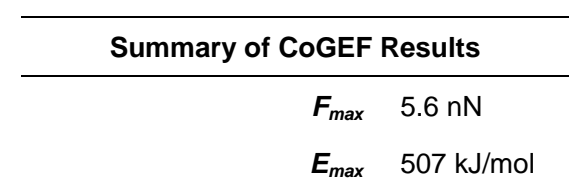

Force-Bond Angle $32^{\circ}$

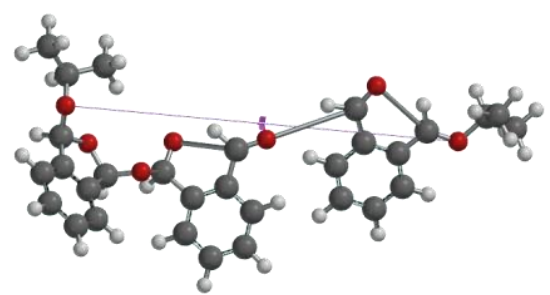

$15.649 \AA$ 

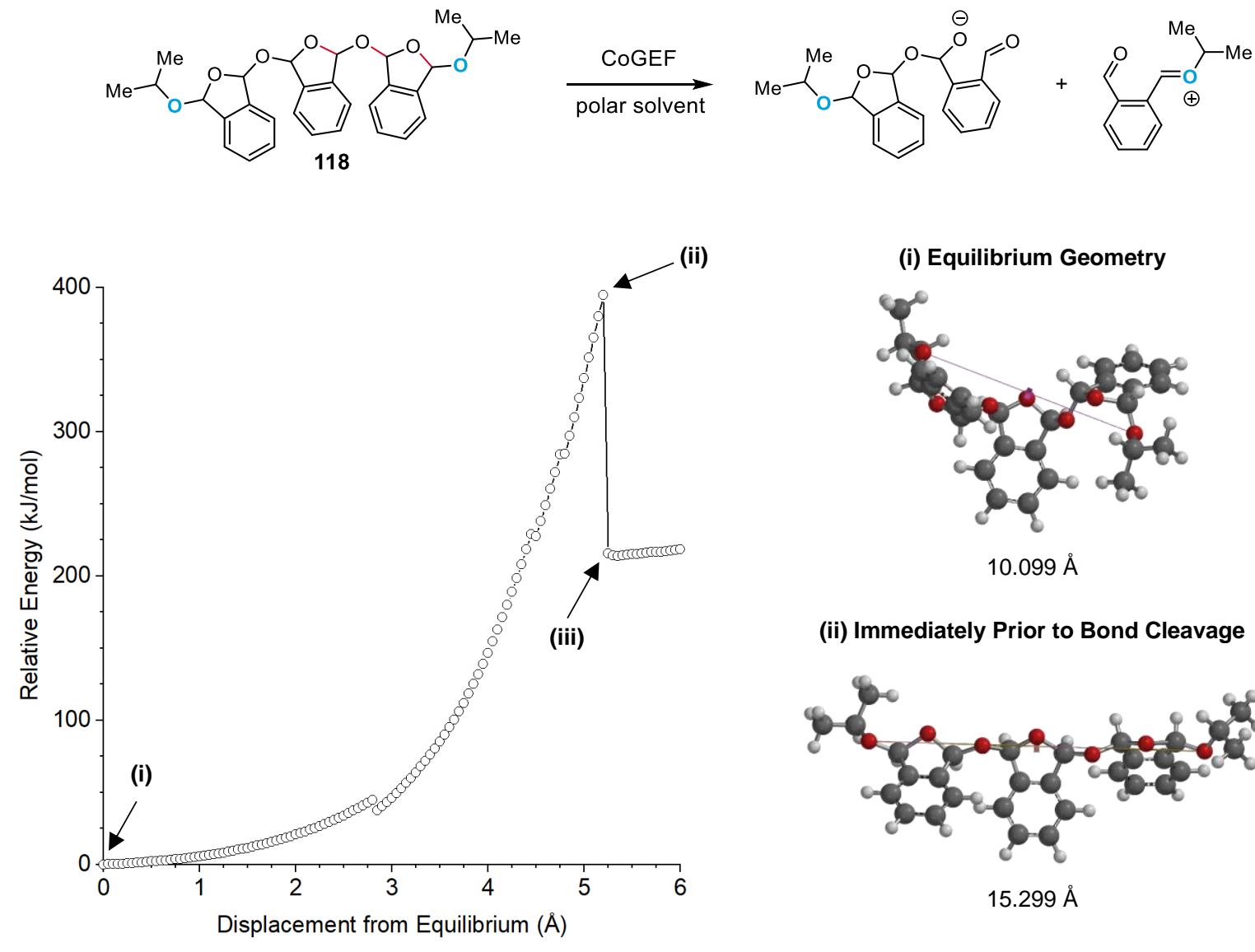

(i) Equilibrium Geometry

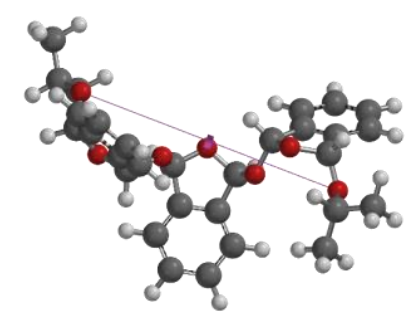

$10.099 \AA$

(ii) Immediately Prior to Bond Cleavage

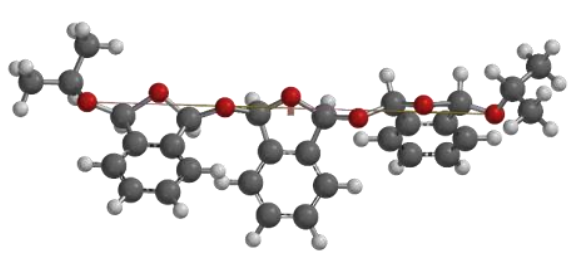

$15.299 \AA$

(iii) Immediately After Bond Cleavage

Summary of CoGEF Results

$$
\begin{array}{ll}
\boldsymbol{F}_{\max } & 4.9 \mathrm{nN} \\
\boldsymbol{E}_{\max } & 395 \mathrm{~kJ} / \mathrm{mol}
\end{array}
$$

Force-Bond Angle $33^{\circ}$
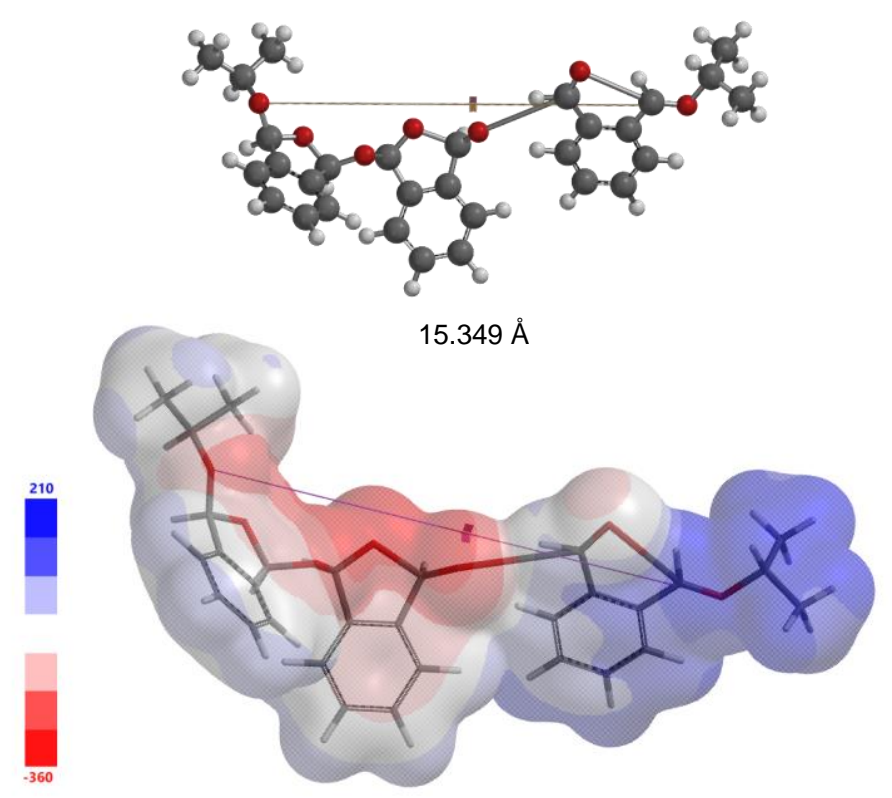


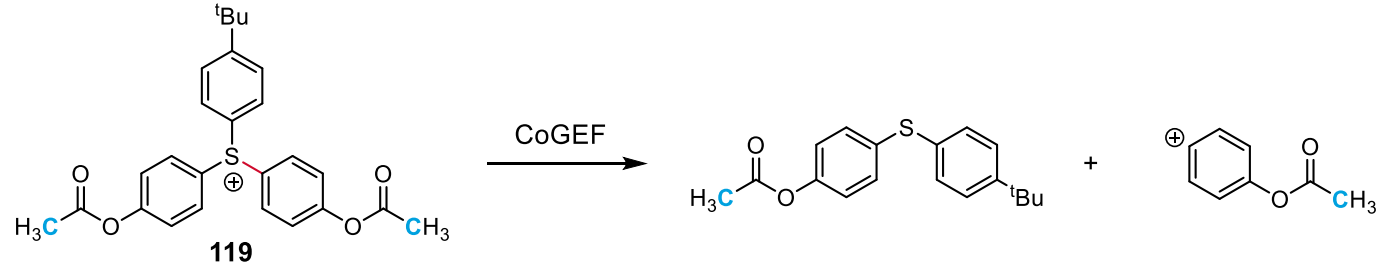

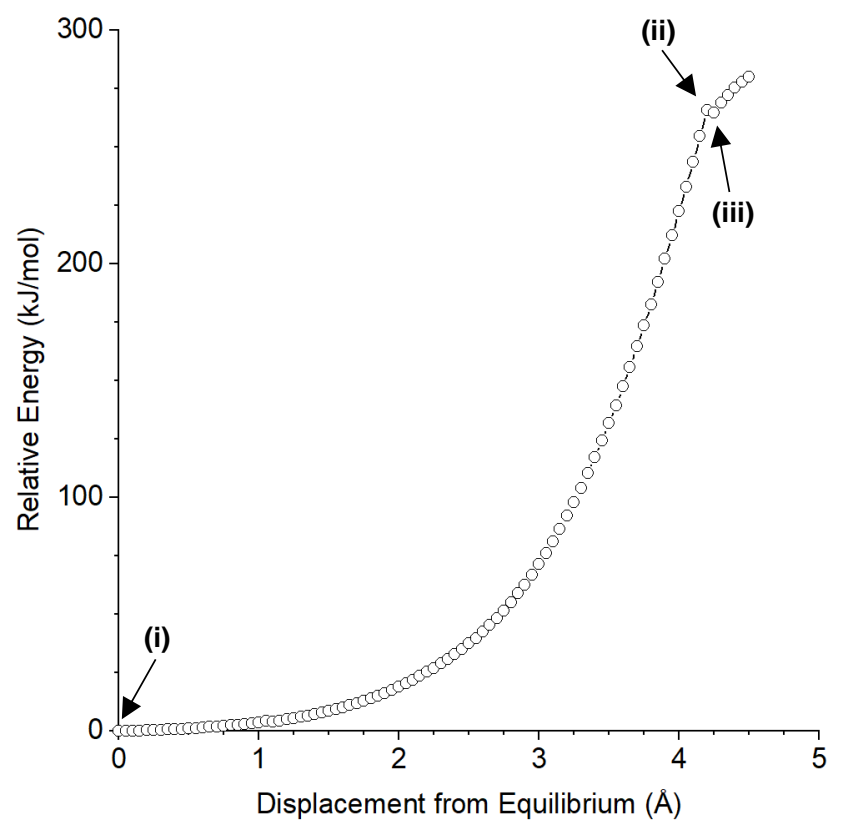

Summary of CoGEF Results

$F_{\max } \quad 3.7 \mathrm{nN}$

$E_{\max } \quad 266 \mathrm{~kJ} / \mathrm{mol}$

Force-Bond Angle $17^{\circ}$

(i) Equilibrium Geometry

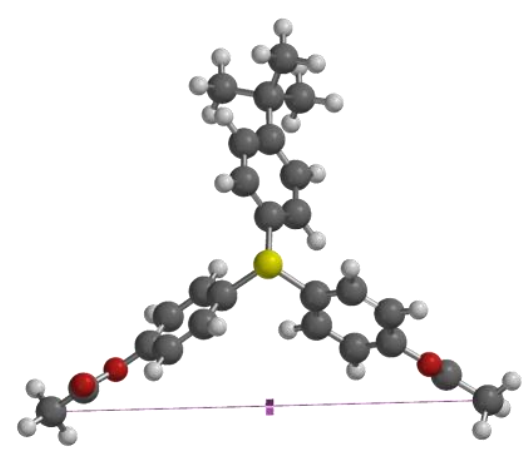

$13.405 \AA$

(ii) Immediately Prior to Bond Cleavage

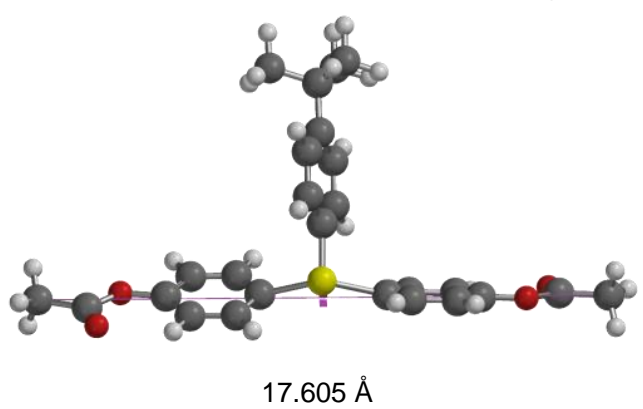

(iii) Immediately After Bond Cleavage

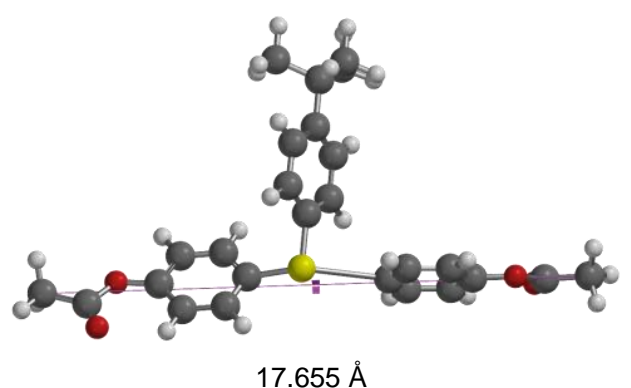




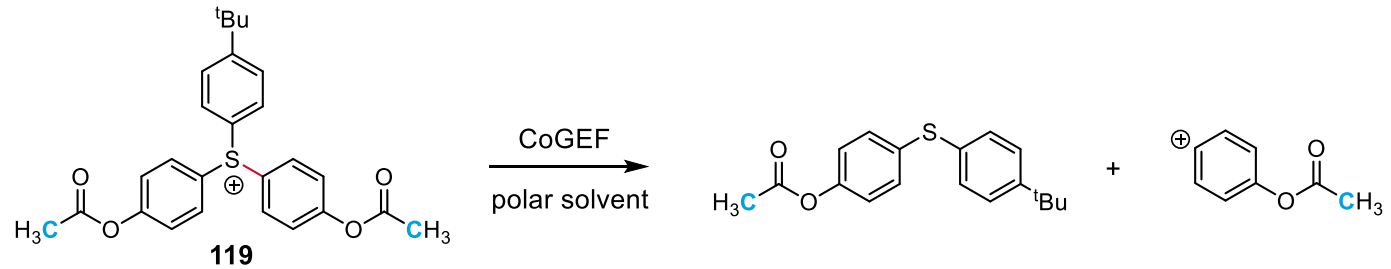

(ii)

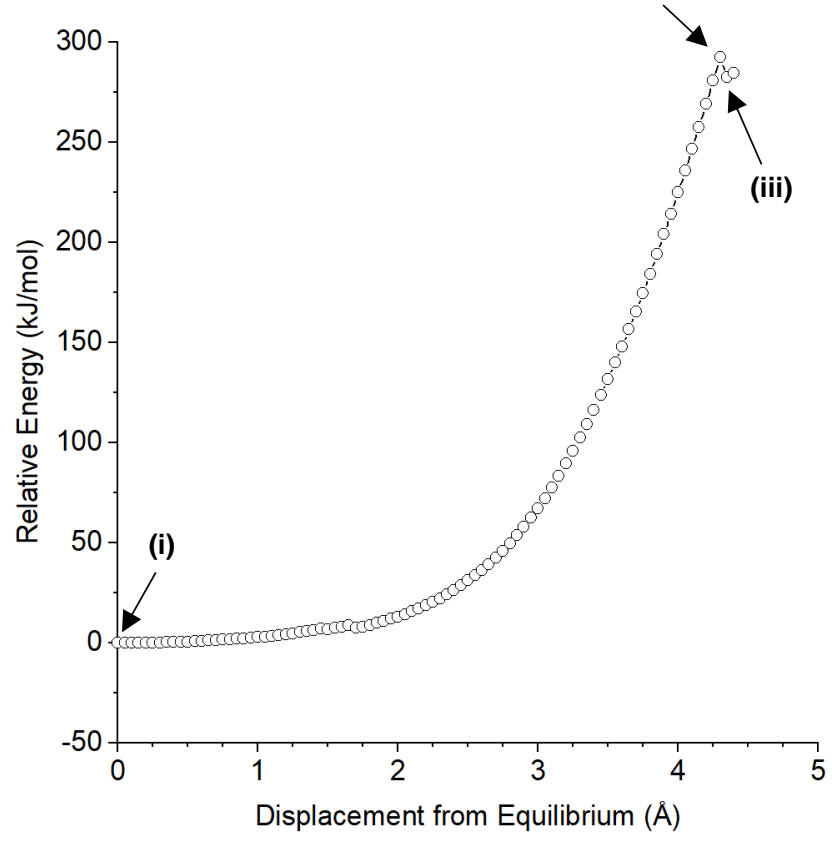

Summary of CoGEF Results

$F_{\max } \quad 3.9 \mathrm{nN}$

$\boldsymbol{E}_{\max } \quad 292 \mathrm{~kJ} / \mathrm{mol}$

Force-Bond Angle $18^{\circ}$

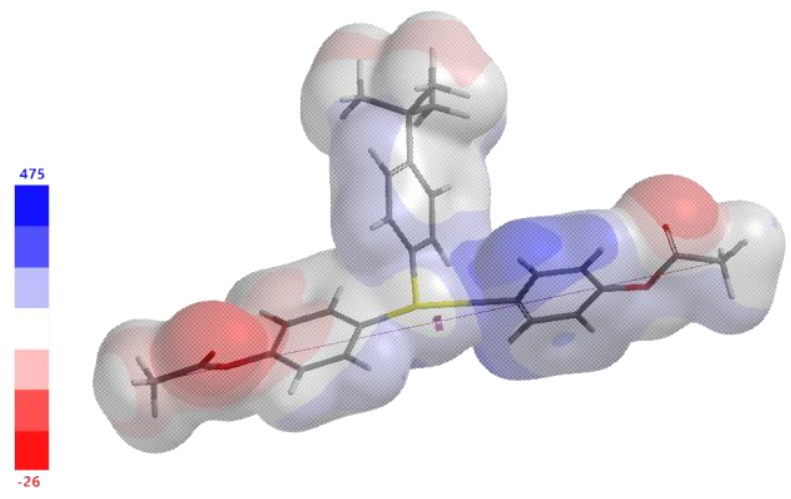

(i) Equilibrium Geometry

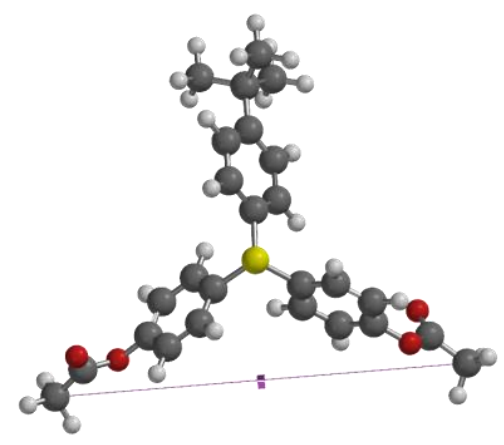

$13.405 \AA$

(ii) Immediately Prior to Bond Cleavage

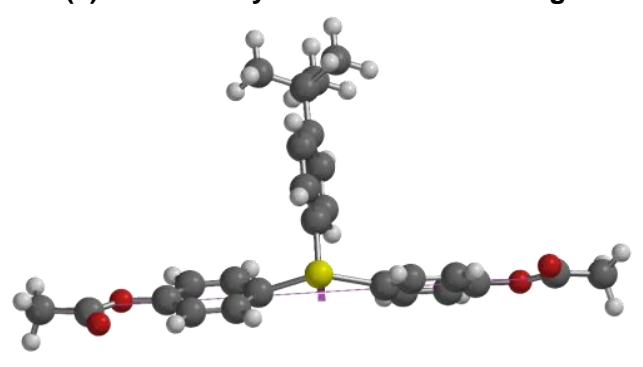

$17.705 \AA$

(iii) Immediately After Bond Cleavage

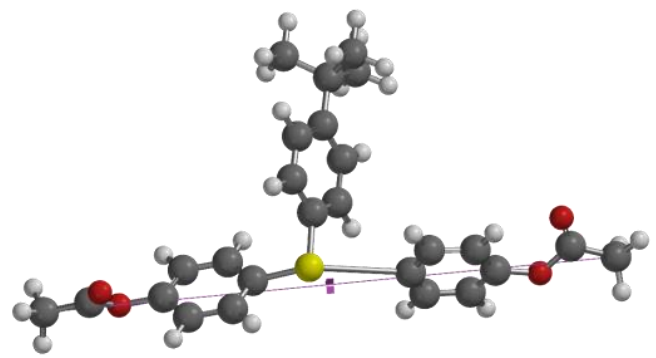

$17.755 \AA$ 
<smiles>CCN(CC)c1ccc2c(c1)Oc1cc(OC(=O)NC)ccc1C21c2ccccc2C(=O)N1CCOC(=O)NC</smiles><smiles>CCC(=O)F</smiles>

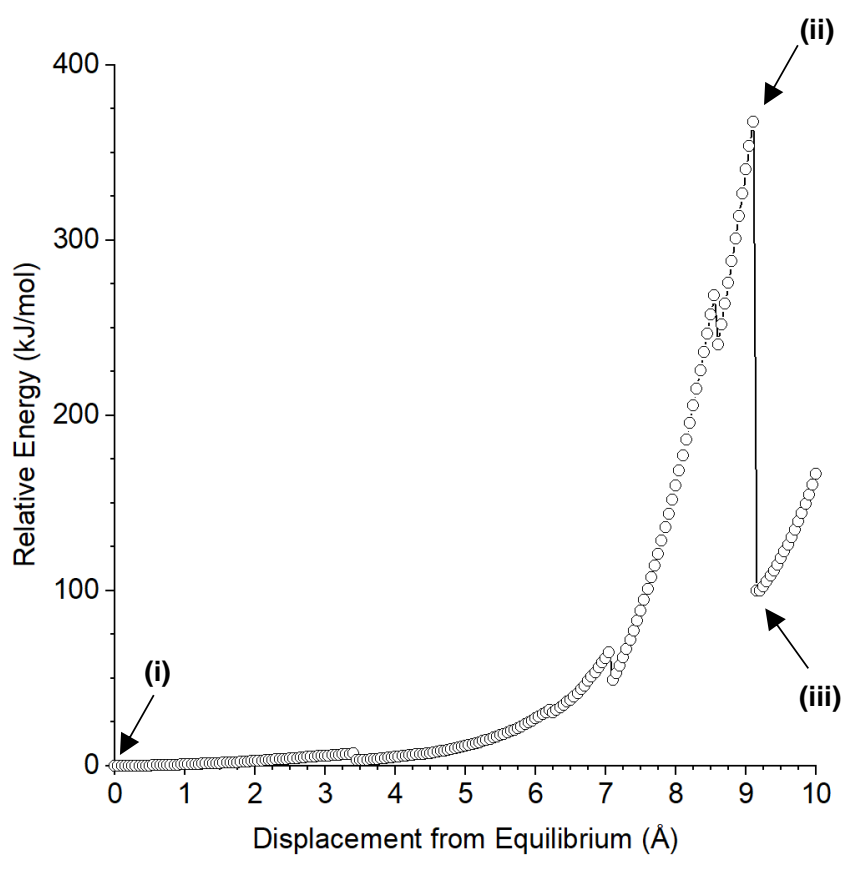

(i) Equilibrium Geometry

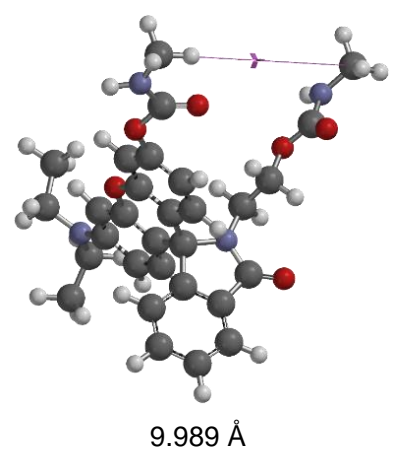

(ii) Immediately Prior to Bond Cleavage

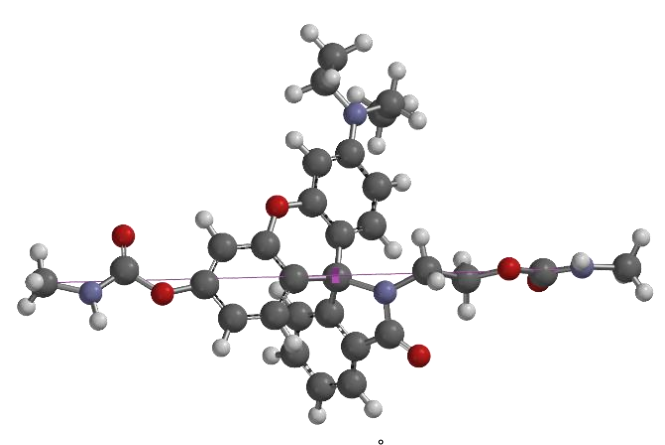

$\boldsymbol{F}_{\max } \quad 4.6 \mathrm{nN}$

$19.089 \AA$

$E_{\max } \quad 368 \mathrm{~kJ} / \mathrm{mol}$

Force-Bond Angle $32^{\circ}$

(iii) Immediately After Bond Cleavage

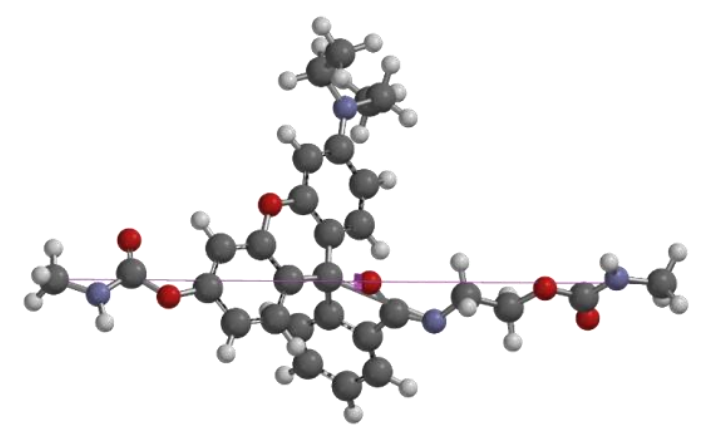

$19.139 \AA$ 
<smiles>CCN(CC)c1ccc2c(c1)Oc1cc(OC(=O)NC)ccc1C21C(=O)N(CCOC(=O)NC)c2ccccc21</smiles>

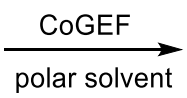

(ii)

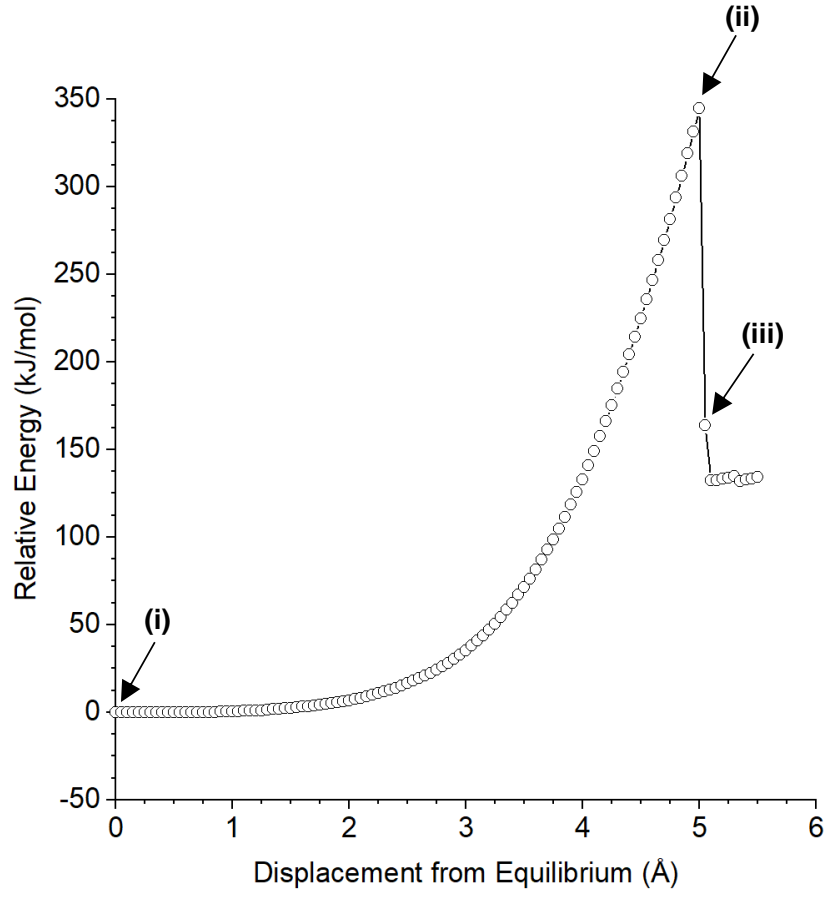

Summary of CoGEF Results

$$
\begin{array}{ll}
\boldsymbol{F}_{\max } & 4.3 \mathrm{nN} \\
\boldsymbol{E}_{\max } & 345 \mathrm{~kJ} / \mathrm{mol}
\end{array}
$$

Force-Bond Angle $32^{\circ}$

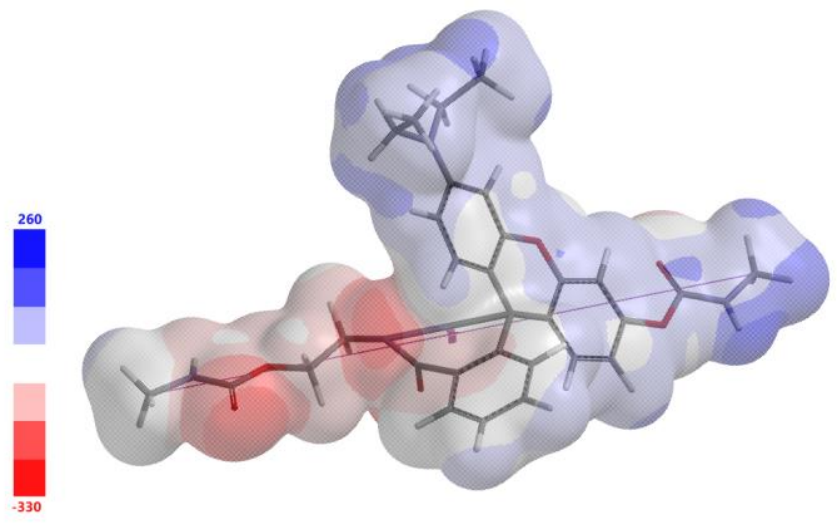<smiles></smiles>

(i) Equilibrium Geometry

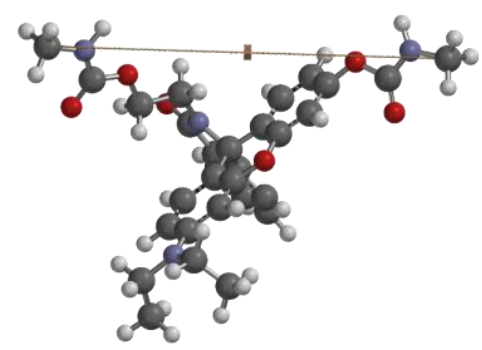

$14.019 \AA$

(ii) Immediately Prior to Bond Cleavage

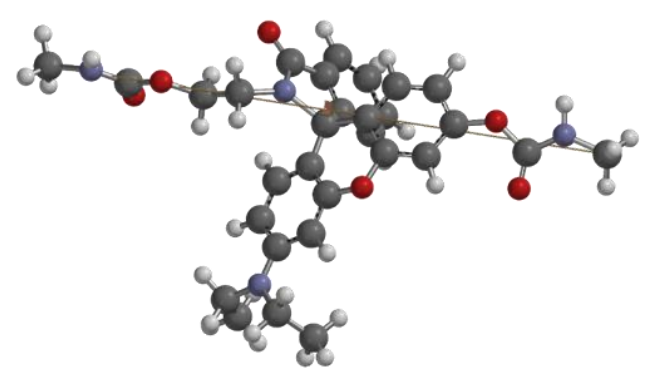

$19.019 \AA$

(iii) Immediately After Bond Cleavage

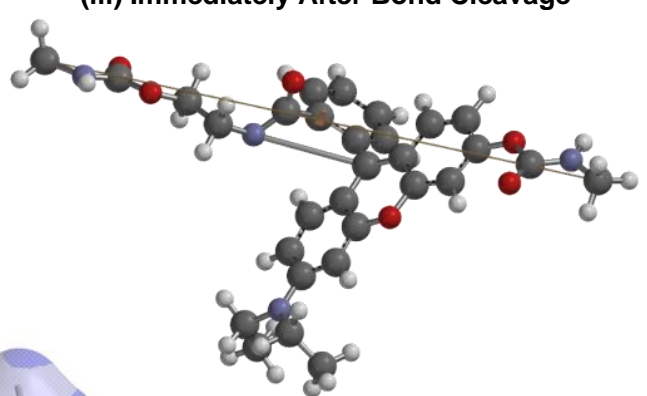

$19.069 \AA$ 